\title{
Centro
}

de Informática

$U \cdot F \cdot P \cdot E$

Pós-Graduação em Ciência da Computação

\section{CONSTRUCTIVE EXTENSIBILITY OF TRUSTWORTHY COMPONENT-BASED} SYSTEMS

By

JOSÉ DIHEGO DA SILVA OLIVEIRA

Ph.D. Thesis

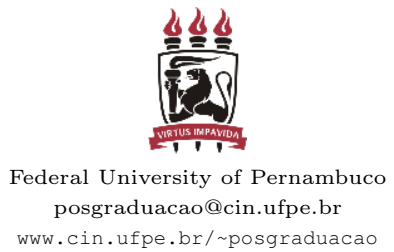

RECIFE/2016 


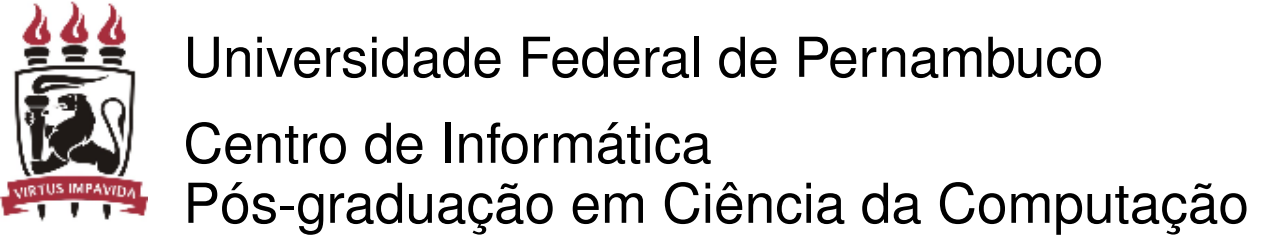

José Dihego da Silva Oliveira

\section{CONSTRUCTIVE EXTENSIBILITY OF TRUSTWORTHY COMPONENT-BASED SYSTEMS}

A Ph.D. Thesis presented to the Center for Informatics of Federal University of Pernambuco in partial fulfilment of the requirements for the degree of Philosophy Doctor in Computer Science.

Advisor: Augusto Cezar Alves Sampaio 
O48c Oliveira, José Dihego da Silva

Constructive extensibility of trustworthy component-based systems / José Dihego da Silva Oliveira. - 2016. 169 f.: il., fig., tab.

Orientador: Augusto Cezar Alves Sampaio.

Tese (Doutorado) - Universidade Federal de Pernambuco. Cln, Ciência da Computação, Recife, 2016.

Inclui referências e apêndices.

1. Engenharia de software. 2. Componentes de software. I. Sampaio, Augusto Cezar Alves (orientador). II. Título.

005.1

$$
\text { CDD (23. ed.) }
$$

UFPE- MEI 2016-078 


\section{José Dihego da Silva Oliveira}

\section{Constructive Extensibility of Trustworthy Component-based Systems”}

Tese de Doutorado apresentada ao Programa de Pós-Graduação em Ciência da Computação da Universidade Federal de Pernambuco, como requisito parcial para a obtenção do título de Doutora em Ciência da Computação

Aprovado em: 04/03/2016.

Orientador: Prof. Dr. Augusto Cézar Alves Sampaio

\section{BANCA EXAMINADORA}

Prof. Dr. Paulo Henrique Monteiro Borba Centro de Informática / UFPE

Prof. Dr. Alexandre Cabral Mota

Centro de Informática / UFPE

Prof. Dr. Nelson Souto Rosa

Centro de Informática / UFPE

Prof. Dr. Christiano de Oliveira Braga

Departamento de Ciência da Computação / UFF

Profa. Dra. Leila Ribeiro

Instituto de Informática / UFRGS 
To my parents, my wife Ivana and my children Clara and Pedro. 


\section{Acknowledgements}

Life is a very short thing, but full of joy if we have a hand to hold. Thank God I have important hands to say thank you, very, very much! My father Genário and mother Lúcia, family farmers, who gave me, by the hard work of their hands (literally), truly love and education. My brothers Igo and Luana, for the hands on slings, for playing in our own forest of banana and coconut trees... awesome.

There is nothing better than to be an innocent child, no money, no bills... Except, if you actually have one, as I have Clara, my little daughter, with her cute and sweet-driven hands! A gift given to me by the love of my life, Ivana, my wife and partner, who has held my hand every day in this journey.

Some people do their duty, others do what is possible, but others, like Augusto Sampaio, makes the world better, pushing the boundaries. A scientist, in the essence of the word, who gives attention and respects to his students and colleagues, an adviser, that have read every word I wrote and help me grown. Thank you very much for such generous hands!

I am indebted to Rodrigo Texeira, who has created the base for this work and answered with patience every question. I am thankful to Marcel Oliveira, Ana Cavalcanti, Pedro Antonino and Madiel Conserva for giving me their time and having been patient to listen and improve my work.

I would like to thank Paulo Borba, Alexandre Mota and Christiano Braga for their edifying comments in my qualification exam, for being there and helped me to achieve a better result.

Finally, I need to say that the Universidade Federal de Pernambuco changed my life: the professors, the staff, the colleagues and the structure are a treasure for Recife and for Nordeste and I am very proud of being part of this history. I would like to thank IFBA for the financial support in the last two years of this journey, to which, I hope to contribute to its entire community. 
vo - ca me cum bene - dic - tis, cum be - ne - dic - tis, vo - ca me, vo - ca me, vo - ca me cum bene - dic —WOLFGANG AMADEUS MOZART 


\section{Resumo}

Na medida em que os sistemas computacionais se tornam mais pervasivos, a demanda por métodos de desenvolvimento rigorosos e composicionais cresce dramaticamente. No desenvolvimento baseado em componentes (CB-MDD), sistemas complexos (muitas vezes humanamente intangíveis) são construídos a partir de elementos mais simples, chamados componentes. Para atingir os objetivos desta abordagem na direção de torná-la uma disciplina formal de desenvolvimento, componentes e regras de composição devem ser formalizados. Além disso, considerando que os requisitos de um sistema estão em constante evolução, necessitamos de mecanismos para refinar e estender de forma confiável tais sistemas. O modelo de componentes BRIC formaliza os conceitos chave da abordagem CB-MDD, além de garantir corretude por construção se baseando em regras de composição que preservam propriedades comportamentais. BRIC, porém, por não possuir relações de extensão, não suporta evolução de modelos baseados em componentes.

Neste trabalho propomos relações de herança e refinamento para BRIC. Definimos uma semântica congruente que considera tanto a estrutura quanto o comportamento de componentes. Definimos refinamento como uma relação de pré-ordem, a qual é monotônica em relação as regras de composição de BRIC. Estendemos este modelo de componentes com suporte a extensibilidade via herança. As relações propostas permitem extensão de funcionalidade, ao mesmo tempo em que preservam conformidade de serviços, a qual é definida em termos de uma noção de convergência. Estabelecemos também uma conexão algébrica entre extensibilidade de componentes e refinamento. Até onde estamos cientes, este trabalho é pioneiro no desenvolvimento de noções de herança de componentes para uma abordagem CB-MDD formal e consistente.

Também integramos o paradigma orientado a aspectos em BRIC. Contribuímos com uma abordagem para capturar, especificar e adotar aspectos no desenvolvimento confiável de sistemas baseados em componentes. Estabelecemos que componentes estendidos por aspectos preservam convergência, o que garante conformidade de serviços. Além disso, desenvolvemos uma conexão entre herança e aspectos, apresentando herança como um mecanismo para definir famílias de componentes e aspectos para capturar conceitos ortogonais sobre as mesmas.

Ilustramos a relevância prática das relações propostas através de três estudos de caso. No primeiro, modelamos um sistema autônomo de cuidados médicos, estendido pela adição de novas funcionalidades via herança e pela modularização de conceitos transversais de forma reusável e manutenível via aspectos. Na sequência, modelamos um sistema bancário, cujas funcionalidades são progressivamente implementadas e estendidas pelo uso de herança e refinamento. Finalmente, modelamos um sistema P2P cujo tráfico é reduzido por extensão via herança.

Palavras-chave: herança de componentes. refinamento de componentes. correção por construção. design orientado a aspectos para modelos de componentes. convergência comportamental. CSP 


\section{Abstract}

As computer systems become ubiquitous, the demand for rigorous and compositional development methods increase dramatically. In the component-based model driven development (CB-MDD) approach, complex systems (sometimes intractable by humans) are build from simple elements, called components. To achieve the CB-MDD goals towards becoming a rigorously development discipline, components and composition rules must be formalised. Moreover, as requirements continuously evolve, there must be mechanisms to refine and safely extend component-based systems. The BRIC component model formalises the CB-MDD core concepts and supports a constructive design based on composition rules that preserves behavioural properties, but do not provide support for component model evolution.

In this work we propose inheritance and refinement relations for BRIC. We define a congruent semantics for this model that considers component structure and behaviour. We define refinement as a preorder relation, which is monotonic with respect to the BRIC composition rules. We enhance this component model with support for extensibility via inheritance. The proposed relations allow extension of functionality, whilst preserving service conformance, which we define by means of a convergence notion. We also establish an algebraic connection between component extensibility and refinement. As far as we are aware this is the first time component inheritance relations are developed for a formal and sound CB-MDD approach.

We also integrate the aspect-oriented paradigm into BRIC. We contribute with an approach to capture, specify and use aspects to safely evolve component-based systems. We establish that components extended by aspects preserve the proposed convergence relation that guarantees service conformance. Furthermore, we establish a connection between component inheritance and aspects, presenting inheritance as a mechanism to define families of components and aspects to capture orthogonal concerns over them.

The practical relevance of the proposed relations is illustrated by three case studies. One is an autonomous healthcare system, which evolve by the addition of new functionalities via inheritance and by the modularisation of its crosscutting concerns in a reusable and maintainable manner with aspects. Another case study is a bank system, whose functionalities are progressively realised and extended by refinement and inheritance, respectively. Finally, we model a P2P system extended by inheritance to reduce the network traffic.

Keywords: component inheritance. component refinement. correctness by construction. component-based aspect-oriented design. behavioural convergence. CSP 


\section{List of Figures}

2.1 STOP, SKIP, prefix and choice . . . . . . . . . . . . . . 21

2.2 Hiding and sequence . . . . . . . . . . . . . . . . 22

2.3 Rename . . . . . . . . . . . . . . . . . . . 22

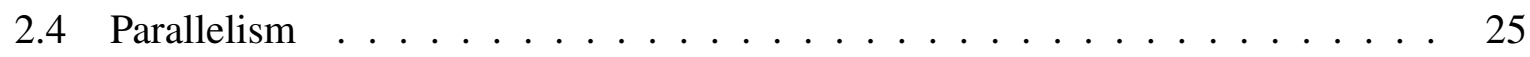

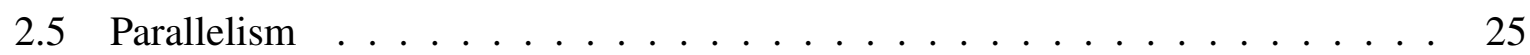

2.6 TV remote control extension . . . . . . . . . . . . . . . 30

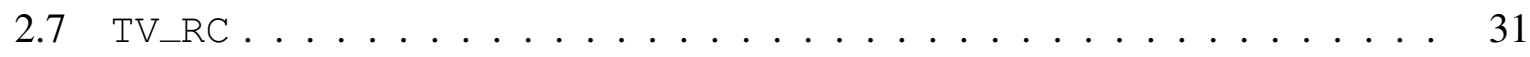

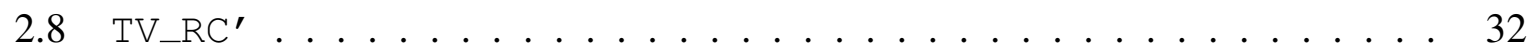

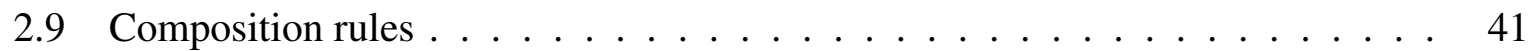

$4.1 \quad \mathrm{I} / \mathrm{O}$ convergent behaviours $\ldots \ldots \ldots \ldots \ldots \ldots$

4.2 I/O non-convergent behaviour . . . . . . . . . . . . . . . . . . . . . 64

$4.3 \mathrm{I} / \mathrm{O}$ extended convergent behaviours $\ldots \ldots \ldots \ldots 6$

4.4 Hierarchy of $\mathcal{B R J C}$ relations . . . . . . . . . . . . . 71

4.5 BRJC inheritance and refinement . . . . . . . . . . . . . . 73

$5.1 \operatorname{Ctr}_{S Y S}=C t r_{A T M}\left[\mathrm{atm \_ cus} \leftrightarrow \mathrm{cus}\right] C t r_{C U S} \ldots \ldots \ldots \ldots 9 . \ldots \ldots$

$5.2 \operatorname{Ctr}_{S Y S 2}=\operatorname{Ctr}_{A T M 2}[\mathrm{~atm} \operatorname{cus} \leftrightarrow \mathrm{cus}] \operatorname{Ctr}_{C U S} \ldots \ldots \ldots \ldots \ldots \ldots$

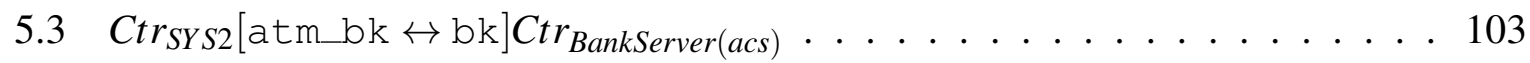

5.4 The healthcare autonomous system hierarchy . . . . . . . . . . . . 111

5.5 Gnutella servent hierarchy . . . . . . . . . . . . . . . . 121

6.1 Marking join points . . . . . . . . . . . . . . . . . 125

6.2 Weaving advices into join points . . . . . . . . . . . . . 128

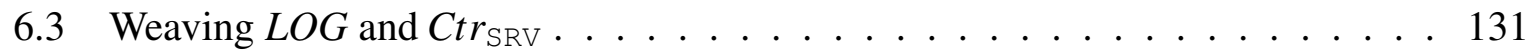

6.4 ACW preserves the healthcare autonomous system hierarchy . . . . . . . . 138 


\section{List of Tables}

C.1 CSP processes . . . . . . . . . . . . . . . . . 168

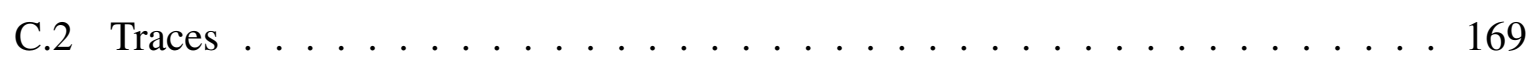

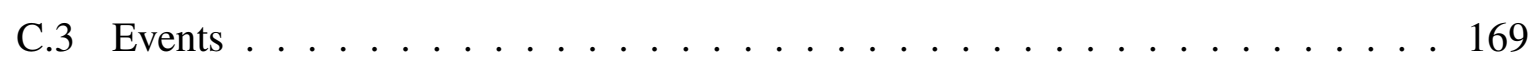




\section{Contents}

1 Introduction $\quad 13$

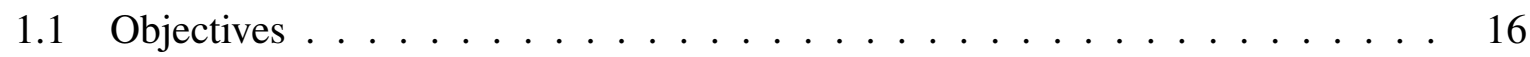

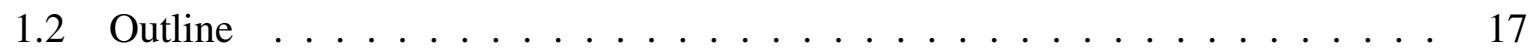

2 Background $\quad 19$

2.1 Communicating Sequential Processes . . . . . . . . . . . . . . . . 19

2.1.1 Traces semantics . . . . . . . . . . . . . . 25

2.1 .2 Failures semantics . . . . . . . . . . . . . 27

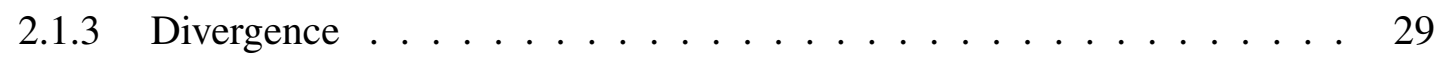

2.2 Motivating example . . . . . . . . . . . . . . . . 30

2.3 The BRIC component model . . . . . . . . . . . . . . . . . 33

2.3.1 Communication protocols . . . . . . . . . . . 35

2.3.2 Asynchronous communication . . . . . . . . . . . . 37

2.3.3 Composition rules . . . . . . . . . . . . . . . . 41

2.4 Component inheritance and refinement . . . . . . . . . . . . . . . . . . . . 44

3 BRIC semantics and refinement $\quad 46$

3.1 BRIC semantics . . . . . . . . . . . . . . . . . . . . . . . 46

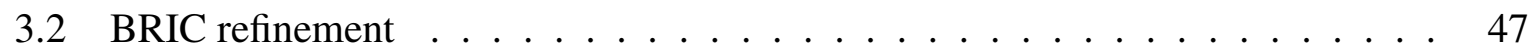

3.3 Monotonicity of BRIC operators with respect to refinement $\ldots \ldots \ldots 5$

$4 \quad$ BRIC inheritance based on convergence $\quad 61$

$4.1 \quad \mathrm{I} / \mathrm{O}$ convergence . . . . . . . . . . . . . . . . . 62

4.1.1 I/O extended convergence . . . . . . . . . . . . . . 64

4.2 BRIC inheritance . . . . . . . . . . . . . . . . . . . 68

4.3 Checking convergence via refinement . . . . . . . . . . . . . . 79

4.3 .1 Building Tester_cvg $($ ecvg $) \ldots \ldots \ldots \ldots$. . . . . . . . 80

5 Case Studies $\quad 96$

5.1 Bank system . . . . . . . . . . . . . . . . . . 96

5.2 An autonomous healthcare robot . . . . . . . . . . . . 104

5.3 P2P: Gnutella protocol . . . . . . . . . . . . . . . . . . 112

6 Aspect-oriented development in BRIC 122

6.1 Aspect-oriented modelling for BRIC . . . . . . . . . . . . . . . . . . 124

6.2 Safely evolving BRIC components using aspects . . . . . . . . . . . . 131 
6.3 Case study . . . . . . . . . . . . . . . . . . . . . . . . . 134

7 Conclusions, related and future work 139

7.1 Contributions . . . . . . . . . . . . . . . . . . . . 139

7.2 Related work . . . . . . . . . . . . . . . . . . . 142

7.3 Future work . . . . . . . . . . . . . . . . . . 144

$\begin{array}{ll}\text { References } & 146\end{array}$

$\begin{array}{ll}\text { Appendix } & 153\end{array}$

A I/O process serialization $\quad 154$

A.1 ATM serialisation . . . . . . . . . . . . . . . . . . . 154

A.2 HC_BOT serialisation . . . . . . . . . . . . . . 160

B Gnutella helper functions $\quad 166$

$\begin{array}{lll}\text { C CSP syntax and notation } & 168\end{array}$ 


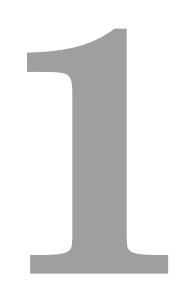

\section{Introduction}

Component-based model driven development (CB-MDD) (SZYPERSKI, 1998) is a well recognised approach to develop complex systems; it has been successfully applied both in industry and academia. The basic idea is that complex systems can be built from simpler units, called components, with well-defined interface and behaviour. Components, interfaces, contracts, implementations and publications constitute the core concepts of CB-MDD. Components are first class entities that can be assembled into more complex ones, until the desired system is developed.

Component-based systems for safety critical applications are required to preserve some desired properties. Of course this has to be achieved in the initial design as well as during the system evolution. Concerning evolution, a natural approach is, in each phase of development, to ensure the model is correct and compliant with respect to the previous versions, by preserving structural and behavioural properties.

In this incremental development one must ensure that new errors are not introduced and classical properties, such as deadlock freedom, are preserved; this can be supported by a notion of component refinement. Refinement notions require a formal semantics that coherently captures the meaning of components. Many formal notations and theories have been developed to support formal specification and refinement, among them, state based (JONES, 1990; WOODCOCK; DAVIES, 1996; LANO, 1996) and event based (ROSCOE, 1998; MILNER, 1989) theories have been developed over the last three decades. Further developments of theorem proving, model checking (FORMAL-SYSTEMS, 2010) and simulation tools have popularised them both in academia and industry.

Component-based development approaches focus on the modelling, analysis, and design phases, trying to ensure that the requirements are properly understood and the abstract system satisfy them. Therefore, the implementation and test phases are alleviated by a proper design, which must, as much as possible, improve reuse.

Some practical approaches have partially adopted the CB-MDD approach by establishing a set of coding conventions to develop executable components with a public interface in their respective programming languages frameworks. Enterprise JavaBeans (EJB) (RUBINGER; 
BURKE, 2010) technology is the server-side component architecture for Java Platform, Enterprise Edition (Java EE); it has been successful both in academia and industry, in part by the large adoption of Java. In the .NET world, COM (Component Object Model) (ROGERSON, 1997; GRAY et al., 1998) is used by developers to create re-usable software components, link components together to build applications in the Microsoft's ecosystem. More formal approaches, as the B-method (ABRIAL et al., 1991) (a constructive approach based on the B Language, refinement and proof), aim to be a platform in which CB-MDD can also be realized.

EJB and COM only address the development phase of CB-MDD, and therefore other complementary techniques/languages must be employed to cope with the analysis and design phases, which are vital in the CB-MDD life cycle. There are two major approaches to carry out system analysis and design: descriptive, diagrammatic or semi-formal, as supported by languages such as UML (RUMBAUGH; JACOBSON; BOOCH, 2004) and UML-RT (Real-Time) (SELIC, 1998) and formal (mathematical unambiguous), such as (CHEN; HE; LIU, 2006; ALLEN; DOUENCE; GARLAN, 1998; HE; LI; LIU, 2006; GEPPERT; TOMBROS; DITTRICH, 1998) and rCOS (HE; LI; LIU, 2006). Critical systems (avionics, nuclear plant powers, financial market) tend to require a formal specification, where entertainment and media applications (games, social networks) require fast prototype/development, are less sensitive to specification errors or omissions and, therefore, they can take advantage of the descriptive approaches.

Architecture description languages (ADLs) have been proposed as modelling notations to support architecture-based development (MEDVIDOVIC; TAYLOR, 2000). They have been designed to address specific domains or to be general purpose modelling languages: Rapide (LUCKHAM et al., 1995), Wright (ALLEN, 1997), Darwin (MAGEE et al., 1995) and Aesop (GARLAN; ALLEN; OCKERBLOOM, 1994) are a short list of dozens ADLs developed in the last twenty years. Each ADL provides complementary capabilities for architectural development and analysis; to understand how they relate and what are the advantages of each approach, the Architecture Description Interchange Language (ACME) (GARLAN; MONROE; WILE, 1997) was developed; it provides a structural framework for characterizing and comparing architectures.

There is some divergence about what an ADL is and how it differentiates from UML or rCOS, for example. Nevertheless, the following definition has recently acquired some consensus (MEDVIDOVIC; TAYLOR, 2000): 'An ADL is a language that provides features for modelling a software system's conceptual architecture, distinguished from the system's implementation'. A system is specified in terms of components, connectors, and architectural configurations. Conversely, it is possible to extend UML to be compliant with the ADL definition (MEDVIDOVIC; ROSENBLUM, 1999); this extension is due to represent architectural abstractions that either differ or do not exist in object oriented design.

ADLs specify how components behave and how they interact to achieve the system requirements. Component behaviour can be specified in terms of state machines, process algebras and logical propositions. Interaction and coordination can be divided among components being part of their behaviour (shared coordination) or mediated by specific-purpose elements, called 
mediators (MEDVIDOVIC; TAYLOR, 2000). Mediators are defined in Reo (ARBAB, 2004), a language for coordination of concurrent processes, which supports the construction of connectors that orchestrate component instances in a component-based system. It is a channel-based coordination model in which complex coordinators, called connectors, are compositionally built out from simpler ones. There are similar works on coordination (ARBAB; RUTTEN, 2003; BONSANGUE et al., 2000), which presents the formal semantics of a kernel of MANIFOLD (ARBAB, 1996), a rigorous coordination language; a categorical semantics is proposed in (FIADEIRO; LOPES, 1997) for the notion of architectural connector (ALLEN; GARLAN, 1994), in which a connector is defined by a set of roles (the expected behaviours of the components being connected) and a glue (how events of the role are coordinated) specification.

The BRJC component model (RAMOS, 2011), based on the CSP (ROSCOE, 1998) process algebra, formalises the core component-based model driven development concepts (SZYPERSKI, 1998) (components, interfaces, channels and behaviour) and, moreover, supports compositions, where deadlock freedom is ensured by construction. This approach covers not only tree topologies, but also cyclic topologies. Although $\mathcal{B} \mathcal{R J C}$ formalises the CB-MDD core concepts it does not provide a semantics for components nor a refinement notion.

Component inheritance arises as a natural aspect to be supported by a CB-MDD approach. It has been a successful feature present in object oriented languages from the beginning (LISKOV; WING, 1994; WEGNER; ZDONIK, 1988; AMERICA, 1991); however, differently from object-orientation, our focus is not on defining subtyping, but extension relations that preserve conformance, and therefore are safe ways to evolve component specifications considering structure and behaviour. Besides reuse, it has the advantage, if proper used, to produce better conceptual designs and can become a way to evolve component systems, alongside refinement, but with a different perspective, as it supports evolution by allowing new functionalities. To achieve this goal in a controlled way, component inheritance must obey the substitutability principle (LISKOV, 1987; WEGNER; ZDONIK, 1988): an instance of the subcomponent should be usable wherever an instance of the supercomponent was expected, without a component, playing the role of a client, being able to tell the difference.

Some works have proposed inheritance relations for behavioural specifications (LISKOV; WING, 1994; AMERICA, 1991; BOWMAN; DERRICK, 1999; PUNTIGAM, 1996; WEHRHEIM, 2003; DIHEGO; ANTONINO; SAMPAIO, 2013). The first four define behaviour in terms of pre/post conditions and structure by method signatures (covariance and contravariance), but do not address these concepts at runtime, as reactive objects. The works reported in (WEHRHEIM, 2003; DIHEGO; ANTONINO; SAMPAIO, 2013), although consider active objects on their formalism, do not focus on structure and, as the others, do not differentiate the nature of input and output events by considering their context; there is a large class of component specifications (such as EJB server, client-server protocol, MVC components) where components control outputs, while the environment (other components) controls inputs. Finally, these works do not consider the impact of these relations over behavioural properties, such as deadlock freedom. 


\section{Objectives}

Our general objective is to contribute to make $\mathcal{B R J C}$ a fully formal approach to component based model driven development. Our approach to achieve this is to define: (a) a congruent semantics for $\mathcal{B} \mathcal{R J C}$ components, complementing the original work of $\mathcal{B} \mathcal{R J C}$ (RAMOS, 2011) (b) a refinement notion and (c) an inheritance relation. These are our main objectives in this thesis.

To define a $\mathcal{B} \mathcal{R J C}$ semantics we need to consider the elements of this component model. The essential parts of a component are its channels (structure) and behaviour (a reactive design expressed in a process algebra), therefore our $\mathcal{B R J C}$ semantics is defined in terms of them, in a compositional way.

A component semantics is essential to define a notion of refinement. This relation must be compositional with respect to the component model operators. It must also be a pre-order (reflexive and transitive). Transitivity supports stepwise development, reflexivity prevents cycles, and compositionality ensures that the relation respects the substitutability principle. In the case of $\mathcal{B R J C}$, as its composition rules ensure preservation of deadlock freedom, this must also be preserved by the refinement relation.

Despite the well-established benefits of a refinement relation, it is restrictive when one considers evolution; the stepwise evolution of a system by means of refinement is, basically, a step towards non-determinism reduction. On the other hand, inheritance, can support a powerful way to evolve systems, by allowing the definition of new functionalities in a conservative manner. It must be capable to satisfy, under certain conditions, the substitutability principle. As refinement, inheritance must consider behavioural aspects and, since $\mathcal{B} \mathcal{R J C}$ distinguishes the nature of input from output events, this relation must cope with this aspect. We propose a behavioural relation called convergence: it captures the idea that components can evolve by accepting new inputs or establishing a communication session after them, but eventually they must be able to converge to the predicted behaviour exhibited by its abstraction.

In practice, both refinement and inheritance only make sense if they can be verified by a model checker, if it is to be adopted in practice and, particularly, at an industrial context. Since BRJE is based on CSP, FDR3 (GIBSON-ROBINSON et al., 2014) is the natural choice given its stability and widespread use in industry and academia. Having a semantics for $\mathcal{B R J \mathcal { C }}$, we illustrate them through the modelling and analysis of some case studies.

Aspect-oriented programming (AOP) (KICZALES et al., 1997) has been presented as a theory to capture, define and modularise crosscutting concerns, which spread through applications. Aspect-oriented programming is part of a broader development strategy, Aspect-Oriented Software Development (AOSD) (FILMAN et al., 2004), which brings aspect-oriented analyses to early phases of software development. The incorporation of aspect-oriented design (AOD) in the modelling phase creates a more accurate and natural correspondence between specification and implementation, benefiting costly development activities as testing and maintenance. A 
promising scenario that we investigate here is the integration of AOSD and component-based model driven development (CB-MDD) (SZYPERSKI, 1998). We contribute by formalising how to capture, model and weave aspects with components in the $\mathcal{B R J \mathcal { C }}$. We use aspects as an important concept to structure the evolution of components.

\section{Outline}

This thesis is organised as follows. In Chapter 2 we define the background theories for this work. Section 2.1 introduces the CSP (HOARE, 1978) (Communicating Sequential Processes) language, presenting its essential constructions and the semantic models used by this work: the failures and traces models; Section 2.3 presents the $\mathcal{B R J C}$ component model by presenting its elements (component contract, I/O process, I/O channels), behavioural properties (input determinism and strong output decisiveness), communication protocols, and its composition rules (interleaving, communication, feedback, and reflexive); Section 2.4 discusses component inheritance and refinement.

Chapter 3 presents our proposed $\mathcal{B R J C}$ semantics and refinement notion. Section 3.1 presents the $\mathcal{B R J C}$ semantics that considers component structure and behaviour. Section 3.2 presents the component refinement relation. It holds the relevant properties required by the $\mathcal{B R J C}$ composition rules, defined in Section 2.3. Section 3.3 shows that refinement is a monotonic relation with respect to interleave, communication, feedback and reflexive compositions.

Chapter 4 defines two inheritance relations for $\mathcal{B R J \mathcal { C }}$, which are based on a novel concept developed in this work called convergence. It captures the idea that components can evolve by accepting new inputs or establishing a communication session after them, but eventually they must be able to converge to the predicted behaviour exhibited by its abstraction. Section 4.1 presents the concept of convergence and its extended version, expressing them in two abstraction levels, traces and failures; Section 4.2 defines our inheritance relations and shows they guarantee substitutability concerning deadlock freedom. We also prove that one is a generalisation of the other and, with refinement, they form a hierarchy; Section 4.3 presents the construction of an automated test for conformance verification of our convergence relations in FDR3 (FailuresDivergence Refinement) (GIBSON-ROBINSON et al., 2014). Finally, Section 5.1 presents a case study of a bank system, where inheritance is used to reduce the non-determinism and to offer realistic services (in a business view). This is an important result that cannot be achieved using refinement alone.

Chapter 5 presents three case studies concerning an autonomous healthcare robot system, a bank system and a P2P network. They are stepwisely improved by the use of refinement to encompass implementation details, and through inheritance to add new functionalities.

In Chapter 6 we stablish how to define and weave aspects into component-based specifications. We also prove that aspects guarantee service conformance by establishing how it relates with component inheritance. We conclude this chapter revisiting the case study of the autonomous healthcare system developed in Chapter 5, which is extended in a modular and 
maintainable way by the adoption of an aspect-oriented design. We conclude this work in Chapter 7 , where we present our contributions, related and future work. 


\section{2}

\section{Background}

This chapter presents the theoretical foundation for this work. The formal treatment of component inheritance demands an unambiguous approach to define component behaviour and structure, as well rules to compose them. The $\mathcal{B R J C}$ component model meets such requirements by supporting the definition of components, connectors, their behaviour and the rules by which they are assembled. In fact, it is an approach to CB-MDD (SZYPERSKI, 1998) (ComponentBased Model Driven Development), where complex systems can be built from simpler ones, called components, with well-defined interface and behaviour. In $\mathcal{B R J C}$, behaviour is modelled in CSP (ROSCOE, 1998) (Communicating Sequential Processes) process algebra. CSP is a specification language and a theory for modelling and analysing concurrent systems. Before presenting the $\mathcal{B} \mathcal{R J C}$ model, we discuss the CSP syntax and semantic models.

\section{Communicating Sequential Processes}

In the 1970s Hoare introduced the language CSP (HOARE, 1978) (Communicating Sequential Processes) and the concept of a system made of a combination of processes, each with its own private set of variables, interacting by sending messages to each other via handshake communication.

As the researches advanced on the study of the CSP language, it naturally evolved to a standard notation (ROSCOE, 1998). The CSP version proposed by Roscoe owes part of its success to FDR (FORMAL-SYSTEMS, 2010) (standing for Failures/Divergences Refinement), a proof and analytic tool, and ProBE (Formal-Systems, 2003) (Process Behaviour Explorer), a visualization and interaction tool. They influenced the way systems are modelled in CSP and their presentation. These tools have led to a restructuring and standardization of the CSP notation itself, whose machine readable representation, $\operatorname{CSP}_{M}$, combines the ASCII version of CSP with an expression language inspired on functional languages like Haskell.

A CSP process is defined in terms of how and what it communicates externally. We call $\Sigma$ the set of all events that a set of related processes might communicate. Events in $\Sigma$ are instantaneous: the time intervals between them are the same (finite) and irrelevant; also, a process 
only performs an event in $\Sigma$ if it and its environment (other processes) agree on that, and if they do, that event or some other event must happen.

When introducing the CSP notation (see Appendix C), we describe the standard syntax for each operator followed by a machine readable version in parentheses; this latter version is the one used by tools like FDR3 (GIBSON-ROBINSON et al., 2014). CSP has two special processes that indicate termination: STOP (STOP) and SKIP (SKIP). Both processes deadlock, i.e., reach a state where they do not perform any action, but SKIP differs from STOP since it communicates a special event named tick $(\checkmark \notin \Sigma)$ before deadlock ( $\checkmark$ is always the final event a process performs). This event marks a successful termination, whereas STOP is generally associated to a failure or an exception.

Given an event $e v \in \Sigma$ and a process $P, e v \rightarrow P(\mathrm{ev}->\mathrm{P})$ is the process that offers $e v$ to is environment and after engaging in $e v$ it behaves like $P$. The event $e v$ is offered until the environment agrees on it, if it does not so, $e v \rightarrow P$ will wait forever.

With the notion we have seen so far a process has a unique way of behaving. If we want to model a process that has more than one possible behaviour alternative we must use one of the CSP choice operators. The process $e v 1 \rightarrow P \square e v 2 \rightarrow Q$ (ev1 $\rightarrow>$ P [ ] ev2-> Q) offers the choice between $e v 1$ and $e v 2(\{e v 1, e v 2\} \subseteq \Sigma)$ to the environment and then behaves like $P$ if they agree on $e v 1$ or $Q$ if on $e v 2$. This choice is called external choice because the process behaves according to the events performed by the environment. On the other hand, the process $P \sqcap Q$ ( $\mathrm{P}|\sim| \mathrm{Q})$ behaves like $P$ or $Q$, but at this time the choice for one of them is taken without the environment engagement, the process can take by itself an invisible $\tau$ (tau) action towards $P$ or $Q$. An interesting situation occurs in $e v \rightarrow P \square e v \rightarrow Q$, even if the environment is ready to do $e v$, the subsequent behaviour is unpredictable, because it is a non-deterministic choice that was taken by the process itself. This previous process has the same behaviour as $e v \rightarrow(P \sqcap Q)$. So we introduce a non-deterministic (internal) choice, even without the explicit use of the $\sqcap$ operator.

Before we present the formalisation of CSP by discussing some of its semantic models, we present the intuition for the CSP constructions in terms of labelled transition systems (LTS). It provides a visual understanding of the language and will support the subsequent semantic discussion. An LTS consists of a non-empty set of states, one among them being the initial state, a set of labels (events) from $\Sigma^{\tau, \checkmark}=\Sigma \cup\{\tau, \checkmark\}$, and a ternary relation between two states (ordered) and an event meaning that the second state can be reached from the first by the occurring of such an event. Therefore we present, in Figure 2.1 the LTSs of the CSP processes we have seen so far:

- STOP on Figure 2.2(a): a process that just terminates $(\Omega)$;

- SKIP on Figure 2.2(b): a process that terminates after performing the success termination event $\checkmark$;

- ev $->$ P on Figure 2.2(c): a process that communicates ev then behaves as P; 
Figure 2.1: STOP, SKIP, prefix and choice

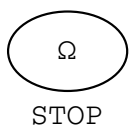

(a)

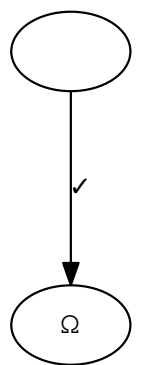

SKIP

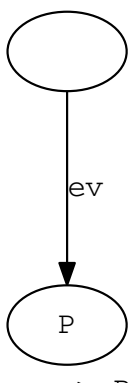

ev $->P$ (b)

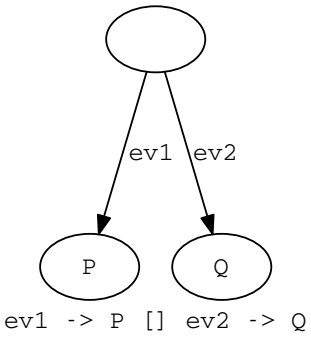

(d)

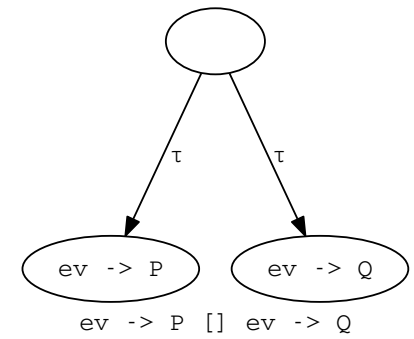

(e) (c)

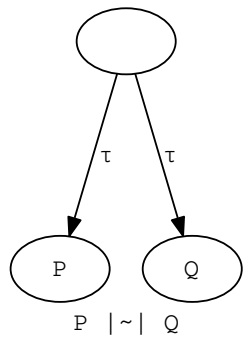

(f)

Source: José Dihego (based on ROSCOE (1998))

- ev1 $\rightarrow$ P [ ] ev2 $->\mathrm{Q}$ on Figure 2.2(d): a process that offers the events ev1 and ev2 in external choice to its environment and behaves as $\mathrm{P}$ if the environment agrees on ev1 or as $Q$ if it agrees on ev2;

- ev $\rightarrow$ P [ ] ev $\rightarrow>Q$ on Figure 2.2(e): a process that behaves as $\mathrm{P} \quad|\sim| \mathrm{Q}$ after communicating ev;

- $\mathrm{P} \quad|\sim| \mathrm{Q}$ on Figure 2.2(f): a process that decides internally to behave as $\mathrm{P}$ or $\mathrm{Q}$.

Hiding implementation details can improve the understanding and the use of a specification. The CSP hiding operator does it by internalising a particular set of events, making them invisible to and uncontrollable by the environment, i.e, turning them into $\tau$. If $P$ is a process and $X \subseteq \Sigma$, then the process $P \backslash X$ ( $\backslash \backslash \mathrm{X}$ ) behaves like $P$ except that the events $X$ are replaced by internal events $(\tau)$. Therefore the process $e v \rightarrow P \backslash X$ can have two different interpretations depending on whether $e v$ is in $X$ (Figure 2.3(a)) or not (Figure 2.3(b)).

All imperative languages provide the sequential composition of commands, in the sense that one particular command is executed only when the previous has finished. In CSP a process finishes if its LTS reaches a $\Omega$ state, but it only do it successfully if $\Omega$ is reached by a $\checkmark$, i.e., if the process eventually behaves as $S K I P$. So, if $P$ and $Q$ are processes, $P ; Q(\mathrm{P} ; \mathrm{Q})$ behaves like 
Figure 2.2: Hiding and sequence

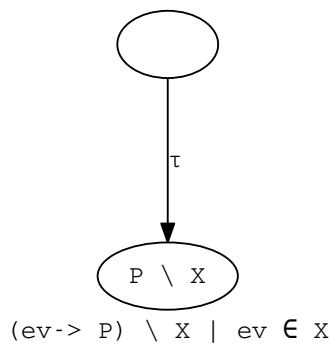

(a)

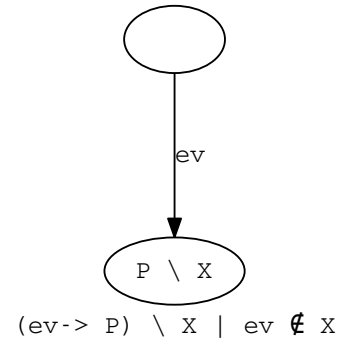

(b)

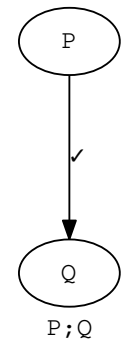

(c)

Source: José Dihego (based on ROSCOE (1998))

Figure 2.3: Rename

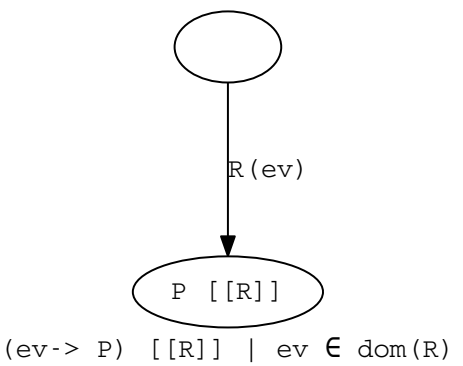

(a)

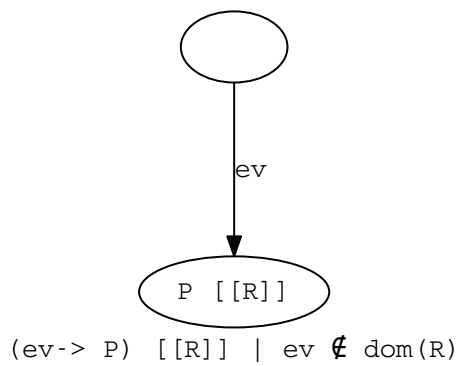

(b)

Source: José Dihego (based on ROSCOE (1998))

$P$ until it terminates successfully and then it behaves like $Q$. If $P$ does not terminate successfully, or does not terminate at all, $Q$ is never executed. It is worth saying that $S K I P ; Q=Q$ and $S T O P ; Q=S T O P$, for any CSP process $Q$, which corroborates our explanation (Figure 2.3(c)).

Consider $R$ a binary relation between elements of $\Sigma$ and $P$ a process, then the process $P[R]](\mathrm{P} \quad[[\mathrm{R}]])$ offers $e v^{\prime}\left(e v R e v^{\prime}\right)$ for every event $e v$ offered by $P$, if $e v \in \operatorname{dom}(R)$ or just $e v$, otherwise. The rename alludes to the hiding notion, but differs from it since it renames a visible event to another, not to $\tau$, as hiding does. If $R$ is a function, then $R(e v)$ has an unique value (when $e v \in \operatorname{dom}(R)$ ), but if it is not the case then $e v^{\prime}\left(e v R e v^{\prime}\right)$ can assume different values, which implies that $e v$ is actually replaced by an internal choice between the possible values of $e v^{\prime}$. The LTS in Figure 2.4(a) captures the case when $e v \in \operatorname{dom}(R)$ ( $R$ is a function), otherwise the behaviour is captured by the LTS in Figure 2.4(b).

Another useful construction of CSP is the conditional choice. It is similar to the respective constructs in procedural languages, but instead of deciding between commands, the condition is evaluated to decide between processes. So if $P$ and $Q$ are two processes and $c$ can be evaluated to a boolean value, the process: if $c$ then $P$ else $Q$ (if c then $\mathrm{P}$ else Q) is $P$ if $c$, otherwise $Q$. 
The CSP notation described so far allows us to define only specifications that have a unique thread of execution. To specify and reason about concurrent systems, CSP supports the definition of processes with independent control flow, interacting with each other synchronously, obeying the handshaking protocol. If $P$ and $Q$ are processes and $X \subseteq \Sigma$, then $P \|_{X} Q(\mathrm{P} \quad[|\mathrm{X}|] \quad \mathrm{Q})$ is called generalised parallel; it is the process where all events in $X$ must be synchronised, and in this way they only can happen if both processes agree to do so. Events outside $X$ can happen independently. Consider the process $e v \rightarrow P \|_{X} e v \rightarrow Q$, so if $e v \in X$ then both processes are ready to engage on $e v$ and after this synchronisation the parallel composition behaves as $P \|_{X} Q$ (Figure 2.5(a)). Otherwise if $e v \notin X$, then the choice of which process will communicate on $e v$ (just one can do it at a time) is non-deterministic (Figure 2.5(b)).

If both processes are ready to communicate, but with different events as in the composition $e v 1 \rightarrow P \|_{X} e v 2 \rightarrow Q$, one of them must be outside $X$, or the process will deadlock (behaving as $S T O P$ ). In Figure 2.6(a) we illustrate the case where $e v 1 \in X$ and $e v 2 \notin X$. In this case, the parallel composition is able to do $e v 2$ independently, but blocks on $e v 1$, since it belongs to the synchronisation set $X$. Finally, if both are outside $X$ the environment is able to pick any of them: they are offered in an external choice (Figure 2.6(b)).

According to Roscoe (ROSCOE, 1998) every process defined with parallelism can be reduced to a sequential one. It is achieved by transformations using a set of algebraic laws and the application of unique fixed point (UFP) rule, when we have recursive processes. By the UFP rule, parallelism is expanded until we have a guarded expression for it (a guarded process always perform some visible event before it recurses, so if $P$ is a CSP process, such that $e v \in \Sigma$, then $P=(e v \rightarrow P) \backslash e v$ is not guarded, but on the other hand $P=e v \rightarrow P$ is guarded) and, every other parallel combination has been discovered during the exploration; therefore, the original process can be fully explained in terms of the discovered guarded processes.

CSP provides other parallel operators, but all can be expressed in terms of $\|_{X}$. Consider $P$ and $Q$ two CSP processes with $X \subseteq \Sigma$ and $Y \subseteq \Sigma$. Then it is possible give the meaning of other parallel operators in terms of generalised parallel.

- (Interleaving) $P\|\| Q(\mathrm{P}|| \mid \mathrm{Q})=P \| Q$ : the processes $P$ and $Q$ behave completely independently of each other. If both can communicate the same event, the choice of which one will be executed is nondeterministic.

- (Alphabetised parallel) $P_{X} \|_{Y} Q\left(\mathrm{P} \quad\left[\begin{array}{lllll}\mathrm{X} & || & \mathrm{Y} & ]\end{array}\right)=P \underset{X \cap Y}{\|} Q: P\right.$ can only communicate events in the set $X$, and $Q$ only those in the set $Y$ but both must agree on events in the intersection $X \cap Y$. This equivalence is only valid if $P$ and $Q$ never communicate outside $X$ and $Y$, respectively; otherwise the meaning in terms of generalised parallelism becomes more subtle, but still possible.

- (Synchronous Parallel) $P\|Q(\mathrm{P} \quad|| \mathrm{Q})=P\|_{\Sigma} Q$ : the processes $P$ and $Q$ must agree 
on all visible events they are willing to communicate, otherwise the composition behaves as $S T O P$.

CSP provides recursion, so if $P=e v \rightarrow P$, then $P$ offers $e v$ indefinitely (thanks to metric spaces and partial orders, recursive guarded processes as $P$ always have a least fixed point (ROSCOE, 1998), so we can calculate their semantics in a finite time). A process can also be parametrised. Consider the process $P(x)$, where $x$ can assume values from the set $T$; if $v \in T$, then $P(v)$ is the process where all occurrences of $x$ are replaced by $v$. Considering prefix, choice and parametrisation we can define processes like $? e v: A \rightarrow Q(e v)$ (the prefix choice operator), which stands by $e v_{1} \rightarrow Q\left(e v_{1}\right) \square e v_{2} \rightarrow Q\left(e v_{2}\right) \square \cdots \square e v_{n} \rightarrow Q\left(e v_{n}\right)$ if $A=\left\{e v_{1}, e v_{2}, \ldots, e v_{n}\right\}$.

If we have a set of processes, especially if it is large one, it can be useful to have indexed versions of the operators that can compose them using external choice, internal choice or parallelism. Therefore if $S$ is a non-empty set of processes, then:

- (Indexed external choice) $\square S$ : offers the processes on $S$ in external choice. Commonly, this operator is indexed by a set of events $X$ in the form $\square_{e v \in X} e v \rightarrow P$ ( [ ] ev:X @ ev $\rightarrow$ P), which stands for $\square\{e v \rightarrow P \mid e v \in X\}$, where $P$ is a CSP process and $X \subseteq \Sigma$;

- (Indexed internal choice) $\Pi S$ : offers the processes on $S$ in internal choice. Similarly to $\square$, this operator can be indexed by a set of events $X$ in the form $\Pi_{e v \in X} e v \rightarrow P$ $(|\sim|$ ev:X @ ev $\rightarrow$ P), which stands for $\bigcap\{e v \rightarrow P \mid e v \in X\}$;

- (Indexed general parallelism) $\|_{X} S$ : offers the processes on $S$ composed in parallel (pairwise), which synchronise on $X$. It is also possible to index parallelism by a subset of $\mathbb{N}$; the process $\|_{X}^{N} P_{i}([|\mathrm{X}|] \quad i:\{1 \ldots \mathrm{N}\}$ @ $\mathrm{P}(\mathrm{i}))$ stands by $P_{1} \|_{X}\left(\ldots\left(P_{N-1} \|_{X} P_{N}\right) \ldots\right)$; therefore, if $N=3$, the composition becomes $P_{1} \|_{X}\left(P_{2} \underset{X}{\|} P_{3}\right)$;

- (Indexed interleaving) $\| \mid S$ : offers the processes on $S$ in interleaving. Similarly to $\|_{X}$ it is possible to index parallelism by a subset of $\mathbb{N}$; the process $\|\left.\right|_{i=1} ^{N} P_{1}$ $(|| \mid i:\{1 \ldots \mathrm{N}\} @ \mathrm{P}(\mathrm{i}))$ is equivalent to $\|_{\emptyset_{i=1}}^{N} P_{i}$.

The events in $\Sigma$ must be grouped into channels, so if $c$ is the name of a channel and $T$ is the type of object communicated by it, we have $c . T=\{c . x \mid x \in T\} \subseteq \Sigma$. It is declared as "channel $c: T$ ". If a channel is declared without a type, as in "channel $c$ ", then it represents the unique event $\{c\} \subseteq \Sigma$.

We have covered most of the CSP language, focusing on the constructs that we use. Now, we are ready to present two of the semantic models for CSP: traces $(\mathcal{T})$ and stable failures $(\mathcal{F})$. 
Figure 2.4: Parallelism
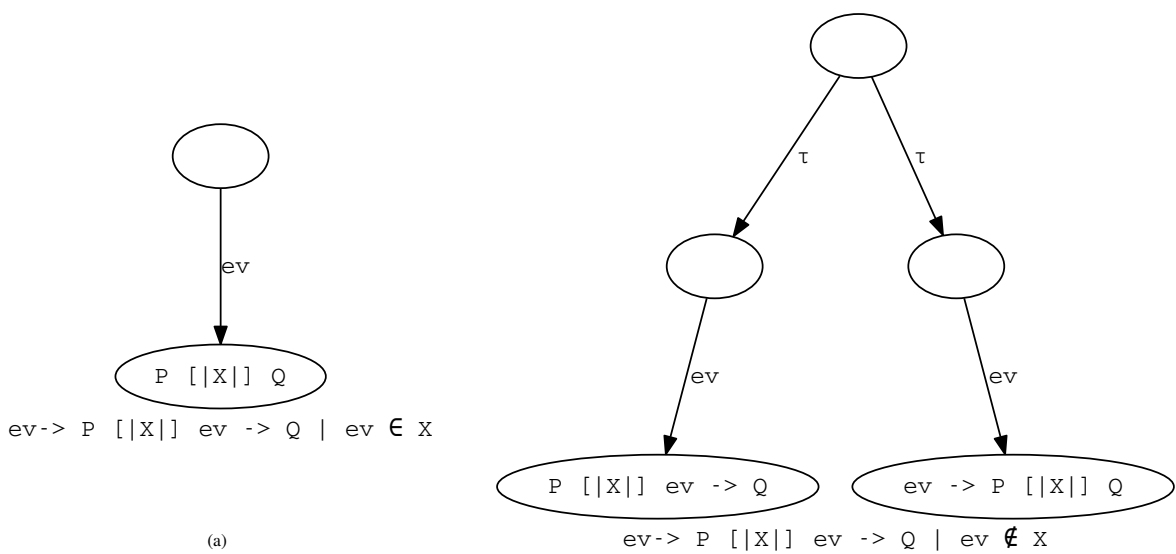

(b)

Source: José Dihego (based on ROSCOE (1998))

Figure 2.5: Parallelism
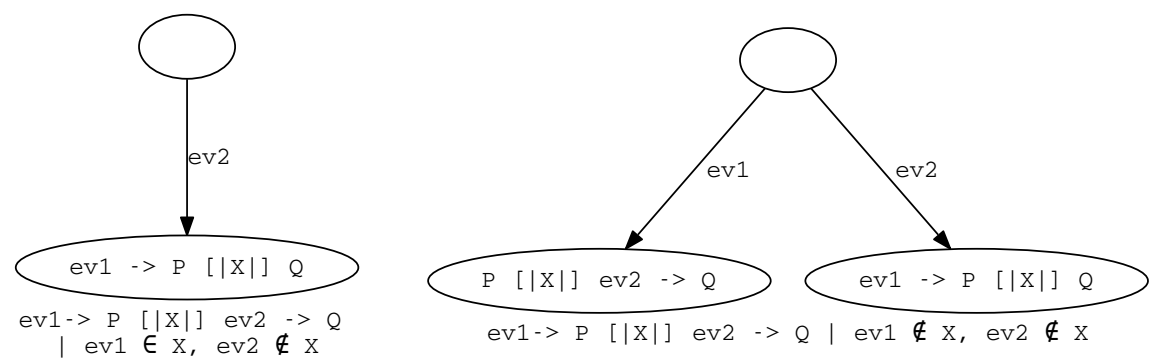

(b)

Source: José Dihego (based on ROSCOE (1998))

\section{Traces semantics}

For any process $P$, we define $\operatorname{traces}(P)$ as the set of all its finite traces, members of $\Sigma^{*}$ (the set of all finite sequences of events in $\Sigma$ ) or its members ended by a $\checkmark$ (no further event is possible after it). This behavioural model satisfies the following healthiness conditions:

- T1. The traces of $P$ are non-empty: \langle\rangle$\in \operatorname{traces}(P)$

- T2. The traces of $P$ are prefix-closed: if $s^{\wedge} t \in \operatorname{traces}(P)$ then $s \in \operatorname{traces}(P)$

Consider the CSP processes $P$ and $Q, X \subseteq \Sigma, e v \in \Sigma$ and $R$ a relation between the elements of $\Sigma$. What follows is the traces semantics ( $\mathcal{T}$ model) of the CSP operators introduced previously. If a construction is not present here, it is because it can be defined in terms of other operators, such as $P\|Q=P\| Q$ : 
- $\operatorname{traces}(S T O P)=\{\langle\rangle\}$. STOP does nothing but terminates.

- $\operatorname{traces}(S K I P)=\{\langle\rangle,\langle\checkmark\rangle\}$. SKIP does a tick, then terminates.

- $\operatorname{traces}(P ; Q)=\left(\operatorname{traces}(P) \cap \Sigma^{*}\right) \cup\left\{s^{\wedge} t \mid s^{\wedge}\langle\checkmark\rangle \in \operatorname{traces}(P) \wedge t \in \operatorname{traces}(Q)\right\}$. It is the union of the traces of $P$ that do not terminate and those that do so concatenated with the traces of $Q$, but without the $\checkmark$.

- $\operatorname{traces}(e v \rightarrow P)=\{\langle\rangle\} \cup\left\{\langle e v\rangle^{\wedge} s \mid s \in \operatorname{traces}(P)\right\}$. The trace $\langle e v\rangle$ is a subtrace of all non-empty traces of $e v \rightarrow P$.

- $\operatorname{traces}(P \square Q)=\operatorname{traces}(P \sqcap Q)=\operatorname{traces}(P) \cup \operatorname{traces}(Q)$. The traces of a choice are the union of the traces of the alternatives. This model does not differentiate external from internal choice.

- $\operatorname{traces}(P \backslash X)=\{s \backslash X \mid s \in \operatorname{traces}(P)\}$. It is the the traces of $P$ where all events $X$ are removed.

- $\operatorname{traces}(P[[R]])=\left\{t \mid \exists s \in \operatorname{traces}(P) \wedge s R^{*} t\right\}$, where $s R^{*} t$ holds if $s$ and $t$ have the same size, and events in the same position of these traces are related by $R$ or are equal.

- $\operatorname{traces}($ if $c$ then $P$ else $Q)=$ if $c$ then $\operatorname{traces}(P)$, otherwise $\operatorname{traces}(Q)$.

- $\operatorname{traces}\left(P \|_{X} Q\right)=\bigcup\left\{\|_{X} t \mid s \in \operatorname{traces}(P) \wedge t \in \operatorname{traces}(Q)\right\}$. The operator $\|_{X}$ (ROSCOE, 1998) over traces combines them in all possible ways but forces them to agree on $X$.

A specification in CSP can be progressively refined towards an implementation in the desired level of abstraction. Therefore, we need a refinement notion to check implementation candidates of a specification. In CSP terms, we need to know when a process refines another.

Definition 2.1 (Trace refinement). If $P$ and $Q$ are processes, then $P \sqsubseteq_{\mathrm{T}} Q \quad(\mathrm{P} \quad[\mathrm{T}=Q)$, read as $Q$ refines $P$, if and only if traces $(Q) \subseteq \operatorname{traces}(P)$.

Definition 2.1 and traces semantics imply the following results, where $P, Q$ and $T$ are arbitrary processes:

- STOP refines anything: $P \sqsubseteq_{\mathrm{T}} S T O P$

- $\sqsubseteq_{\mathrm{T}}$ is transitive: $P \sqsubseteq_{\mathrm{T}} Q \wedge Q \sqsubseteq_{\mathrm{T}} T \Rightarrow P \sqsubseteq_{\mathrm{T}} T$

- $\sqsubseteq_{\mathrm{T}}$ is monotonic: $P \sqsubseteq_{\mathrm{T}} Q \Rightarrow C[P] \sqsubseteq_{\mathrm{T}} C[Q]$, where $C[\cdot]$ is a context (function on processes).

- $\sqsubseteq_{\mathrm{T}}$ is anti-symmetric: $P \sqsubseteq_{\mathrm{T}} Q \wedge Q \sqsubseteq_{\mathrm{T}} T \Rightarrow T \equiv_{\mathrm{T}} Q$. 


\section{Failures semantics}

Although the traces model tracks process execution, it has some limitations. For example, any process is refined by STOP and the internal and external choice between processes produce both the same traces, which makes them indistinguishable. The traces model is restricted to the observation of safety properties. In order to be able to formalise and analyse liveness properties, we need to record not only what a process can do, but also what it can refuse to do (accept to do in a complementary view) in any stable state of its execution. A stable state is one without $\checkmark$ or $\tau$ leading out of it. This means that the unique way of leaving such state is by an event in $\Sigma$ (the set of visible events). The stable failures model registers for each stable state, the set $X \subseteq \Sigma^{\checkmark}$ (and its subsets) of events it can refuse to do from there, however long they are offered.

Every process $P$, in the stable failures model $\mathcal{F}$, is characterised by a pair containing its failures and traces $\mathcal{F}(P)=($ failures $(P)$, traces $(P))$, such that the healthiness conditions $\mathbf{T} 1$ and T2 are satisfied by $\operatorname{traces}(P) \subseteq \Sigma^{* \checkmark}$ and failures $(P) \subseteq \Sigma^{* \checkmark} \times \mathcal{P}\left(\Sigma^{\checkmark}\right) . \mathcal{F}(P)$ satisfies:

- F1. $\operatorname{dom}(\mathcal{F}(P)) \subseteq \mathcal{T}(P)$. Every trace in a failure pair is obviously a trace.

- F2. $(s, X) \in \mathcal{F}(P) \wedge Y \subseteq X \Rightarrow(s, Y) \in \mathcal{F}(P)$. If a process can reject the events in $X$, it can reject any of its subsets.

- F3. $(s, X) \in \mathcal{F}(P) \wedge\left(Y \cap\left\{x \mid s^{\wedge}\langle x\rangle \in \mathcal{T}(P)\right\}=\emptyset\right) \Rightarrow(s, X \cup Y) \in \mathcal{F}(P)$. If an event can never happen after some trace $s$, than it is refused.

- F4. $s^{\wedge}\langle\checkmark\rangle \in \mathcal{T}(P) \Rightarrow\left\{(s, \Sigma),\left(s^{\wedge}\langle\checkmark\rangle, \Sigma^{\checkmark}\right)\right\} \subseteq \mathcal{F}(P)$. If a process can terminate, it can refuse to do anything else but terminate, and after $\checkmark$ it refuses everything $\left(\Sigma^{\checkmark}\right)$.

Let $P$ and $Q$ be CSP processes, $Y \subseteq \Sigma, e v \in \Sigma$ and $R$ a relation between the elements of $\Sigma$. What follows is the stable failures semantics ( $\mathcal{F}$ model) of the CSP operators introduced previously. Following the same pattern adopted for the traces semantics, if a construction is not present here it can be given in terms of other CSP operators.

- failures $(S T O P)=\left\{(\langle\rangle, X) \mid X \subseteq \Sigma^{\checkmark}\right\}$. STOP rejects everything.

- failures $(S K I P)=\{(\langle\rangle, X) \mid X \subseteq \Sigma\} \cup\left\{(\langle\checkmark\rangle, X) \mid X \subseteq \Sigma^{\checkmark}\right\}$. SKIP rejects everything except tick and then becomes STOP.

- failures $(P ; Q)=\left\{(s, X) \mid s \in \Sigma^{*} \wedge(s, X \cup\{\checkmark\}) \in\right.$ failures $\left.(P)\right\} \cup\left\{\left(s^{\wedge} t, X\right) \mid s^{\wedge}\langle\checkmark\rangle \in\right.$ $\operatorname{traces}(P) \wedge(t, X) \in$ failures $(Q)\}$. These are the failures of $P$ before $P$ can successfully terminate. If it can, after some $s$, it raises the failure $\left(s^{\wedge} t, X\right)$ where $(t, X) \in$ failures $(Q)$, in the sense that $t$ happens after $s^{\wedge} \checkmark$ and the whole process refuses the same as $Q$ after $t$. 
- failures $(e v \rightarrow P)=\{(\langle\rangle, X) \mid e v \notin X\} \cup\left\{\left(\langle e v\rangle^{\wedge} s, X\right) \mid(s, X) \in\right.$ failures $\left.(P)\right\}$. In its initial state $e v \rightarrow P$ rejects everything except $e v$; then it has the same failures as those of $P$.

- failures $(P \sqcap Q)=$ failures $(P) \cup$ failures $(Q)$. The initial state of $P \sqcap Q$ is unstable because there are $\tau$ s leading to $P$ or $Q$, therefore failures $(P)$ or failures $(Q)$ are the possible evaluations for failures $(P \sqcap Q)$.

- failures $(P \square Q)=\{(\langle\rangle, X) \mid(\langle\rangle, X) \in$ failures $(P) \cap$ failures $(Q)\} \cup\{(\langle\rangle, X) \mid X \subseteq \Sigma \wedge$ $\langle\checkmark\rangle \in \operatorname{traces}(P) \cup$ traces $(Q)\} \cup\{(s, X) \mid(s, X) \in$ failures $(P) \cup$ failures $(Q) \wedge s \neq\langle\rangle\}$. In its initial state, this choice can refuse an event if both of its branches do so, but if any of them can terminate successfully, it can refuse to do anything but terminate. If a branch is taken, it was by an event in $\Sigma$, which makes the failures of the choice to be that of such a branch.

- failures $(P \backslash X)=\{(s \backslash X, Y) \mid(s, X \cup Y) \in$ failures $(P)\}$. The hiding of $X$ in $P$ replaces them by $\tau$, which can make some of its states unstable. Therefore, we are interested only in $P$ 's states that refuse at least the whole $X$ (it can refuse $Y$ along with $X)$. If such a state is reached by performing the trace $s$, then $(s \backslash X, Y)$ is a failure of $P \backslash X$, since hidden events cannot be refused nor appear in traces.

- failures $(P[[R]])=\left\{(t, R(X)) \mid \exists\right.$ s.s $R^{*} t \wedge(s, X) \in$ failures $\left.(P)\right\}$. The failures of $P[[R]]$ are obtained from those of $P$ by applying $R$ to their traces: $R^{*} s$ maps each event in $s$ according to $R$; and refusals: $R(X)$ gives the set where each $e v \in X$ is mapped to $R(e v)$. if $R$ is not a function, a failure of $P$ can result in more than one failure for $P[[R]$.

- failures (if $c$ then $P$ else $Q)=$ if $c$ then failures $(P)$, otherwise failures $(Q)$.

- $\operatorname{failures}\left(P \|_{X} Q\right)=\{(u, Y \cup Z) \mid \exists s, t .(s, Y) \in \operatorname{failures}(P) \wedge(t, Z) \in \operatorname{failures}(Q) \wedge$ $\left.Y \backslash X^{\checkmark}=Z \backslash X^{\checkmark} \wedge u=s \|_{X} t\right\}$, where $X^{\checkmark}=X \cup\{\checkmark\}$. If $s \in \operatorname{traces}(P)$ and $t \in \operatorname{traces}(Q)$, the combination $P \|_{X} Q$ has the traces $s \|_{X} t$, and after any of them it rejects what can be rejected by $P$ after $S$ (say, $Y$ ) and by $\stackrel{X}{Q}$ after $t$ (say, $Z$ ), but only if $Y$ and $Z$ are equal except for $X^{\checkmark}$ (the synchronisation set plus $\checkmark$ ). If it is not the case, we know that one process can reject an event that the other can accept, so the parallel combination cannot reject such event. If $P$ or $Q$ can reject an event in $X$ it can be rejected by $P \|_{X} Q$, but the others can only be rejected if both processes can also reject them. The $\checkmark$ event has a special treatment: as $X$ events, it can be rejected if $P$ or $Q$ can reject it; on the other hand $\checkmark$ can be communicated by $P$ or $Q$ any time they want. The process $P \|_{X} Q$ terminates only if both terminate, not necessarily at the same time. This is known as distributed termination. 
The failures semantics distinguishes processes by what they communicate and can refuse at stable states. Therefore, the notion of refinement in this model can be viewed as a restriction to the traces model refinement.

Definition 2.2 (Stable failures refinement). If $P$ and $Q$ are processes, than $P \sqsubseteq_{\mathrm{F}} Q$ if and only if $\operatorname{traces}(Q) \subseteq \operatorname{traces}(P)$ and failures $(Q) \subseteq$ failures $(P)$.

By Definition 2.2, for any processes $P$ and $Q, Q$ can neither accept nor refuse an event unless $P$ does, so failures refinement $\left(\sqsubseteq_{\mathrm{F}}\right)$ implies traces refinement $\left(\sqsubseteq_{\mathrm{T}}\right)$ : if $P \sqsubseteq_{\mathrm{F}} Q(\mathrm{P} \quad[\mathrm{F}=\mathrm{Q})$ then $P \sqsubseteq_{\mathrm{T}} Q(\mathrm{P} \quad[\mathrm{T}=\mathrm{Q})$. An import property used throughout this work is that every process whose semantics can be given in $\mathcal{F}$ has an LTS representation (ROSCOE, 1998). Considering this we can state, in very simple words, an important behavioural properties that can be exhibited by CSP processes (in fact by the systems they model), deadlock freedom.

Deadlock. If $P$ is a CSP process then it can deadlock if a $\Omega$ state (it denotes any process that already has terminated) can be reached in its LTS (constructed from $\mathcal{F}(P)$ ). This is equivalent to say that $P$ includes in its definition STOP or SKIP if we avoid the alphabetised parallel operators. This happens because it is impossible to create a process that can deadlock by combining deadlock-free processes without using alphabetised parallelism. Of course we are interested in proving that our specification is responsive, always ready to engage with the environment, i.e., deadlock-free. In semantic terms a process $P$ is deadlock-free $(P$ : [deadlock free]) if $\Sigma^{\checkmark} \notin \operatorname{ran}($ failures $(P))$, where $\operatorname{ran}($ failures $(P)=\{X \mid(s, X) \in \mathcal{F}(P) \wedge s \in \mathcal{T}(P)\}$; so the process never reaches a stable state where it can reject everything. A process that can terminate is not deadlocked, but will be after terminating.

\section{Divergence}

If a process $P$ can perform an infinite and unbroken sequence of internal events we say that $P$ might diverge. If $P$ 's LTS is finite, divergence can only happen if we can find a loop of $\tau$ actions, otherwise it has an infinite LTS with an unbounded branch of $\tau \mathrm{s}$. The set divergences $(P)$ stands for the set of all traces after witch $P$ can diverge, and their arbitrary extensions, since if $P$ reaches a state where it might diverge, we do not try to distinguish what else $P$ might do, so it can do anything. This assumption is called divergence strictness. The recognised, but not exhaustive, sources of divergence are hiding and internal communications made by processes in alphabetised parallelism. Hiding might cause divergence because it renames events from $\Sigma$ to $\tau$ and, thus, from the environment perspective, nothing is happening in a network that does $\tau \mathrm{s}$ forever.

The failures-divergence model $\mathcal{N}$ (and the refinement relation upon it $\sqsubseteq_{\mathrm{FD}}$ ) was proposed to take care of divergences and is a step forward to distinguish between processes otherwise equal in the failures model. Nevertheless, the BRJC component model considers only behavioural specifications that are divergent free (RAMOS, 2011) (the divergences set is empty), which makes $T \sqsubseteq_{\mathrm{FD}} Q$ equivalent to $T \sqsubseteq_{\mathrm{F}} Q$, for any processes $P$ and $Q$; this restriction is essential 
Figure 2.6: TV remote control extension

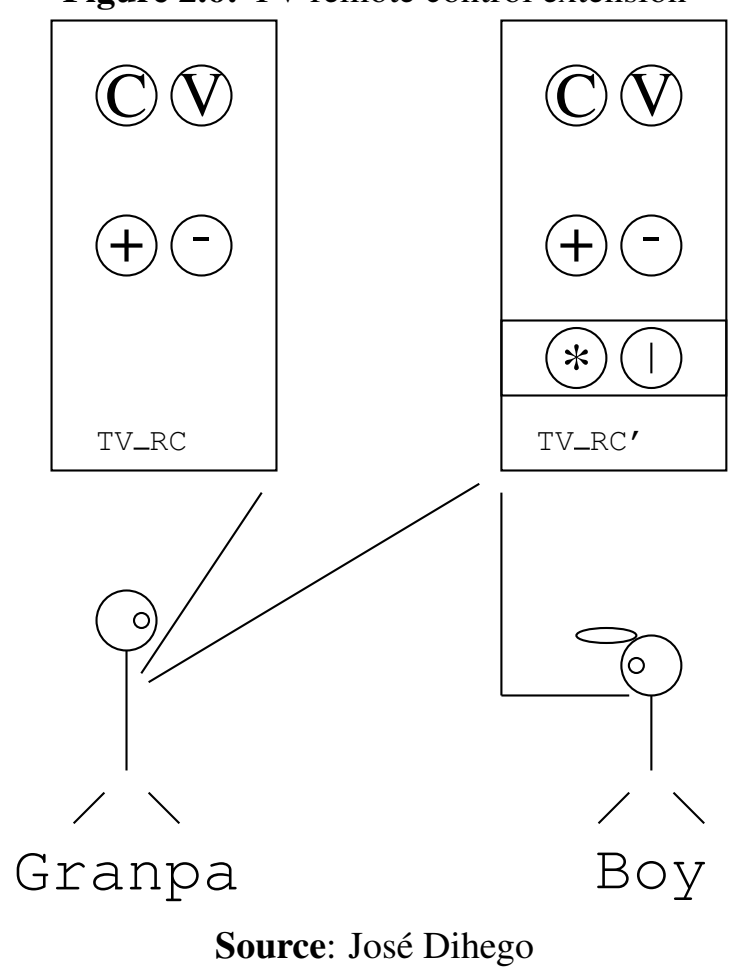

in $\mathcal{B R J C}$ because composition rules assume some degree of predictability about what two components being plugged can communicate to each other, which is impossible if we allow divergent behaviour in $\mathcal{B R J \mathcal { C }}$, the most unpredictable CSP process. Therefore, from now on the failures model $\mathcal{F}$, and its associated refinement relation $\sqsubseteq_{\mathrm{F}}$, will be used as our standard semantics and refinement relation for CSP processes.

\section{Motivating example}

In the next section we present the $\mathcal{B R J C}$ component model, on which we define notions for component inheritance based on convergence (Chapter 4). Given the background on CSP, we are ready to present a short introduction to convergence (formally presented in Section 4.2) via a motivating example; we discuss how it relates to other behavioural relations and why it is relevant in practice.

Consider a TV remote control that offers the options for increase/decrease the TV audio volume and switch between channels. It is represented by the device TV_RC in Figure 2.6; the user Granpa pushes the button (C), then he can go forward or backward on the channel list by pushing $\oplus$ or $\odot$, respectively. The same reasoning applies when Granpa wants to increase/decrease the TV volume by pushing $(\mathrm{V})$. The user Boy wants to do more by having the option to adjust the TV brightness and contrast by pushing the buttons $\circledast$ and (1), respectively. The user Boy is aware of a lid on the device TV_RC' (Figure 2.6), by which he can access these new functionalities, which are completely ignored by Granpa.

We specify the behaviours of $T V \_R C$ and $T V \_R C$ ' in the LTSs depicted in Figures 2.7 
Figure 2.7: TV_RC

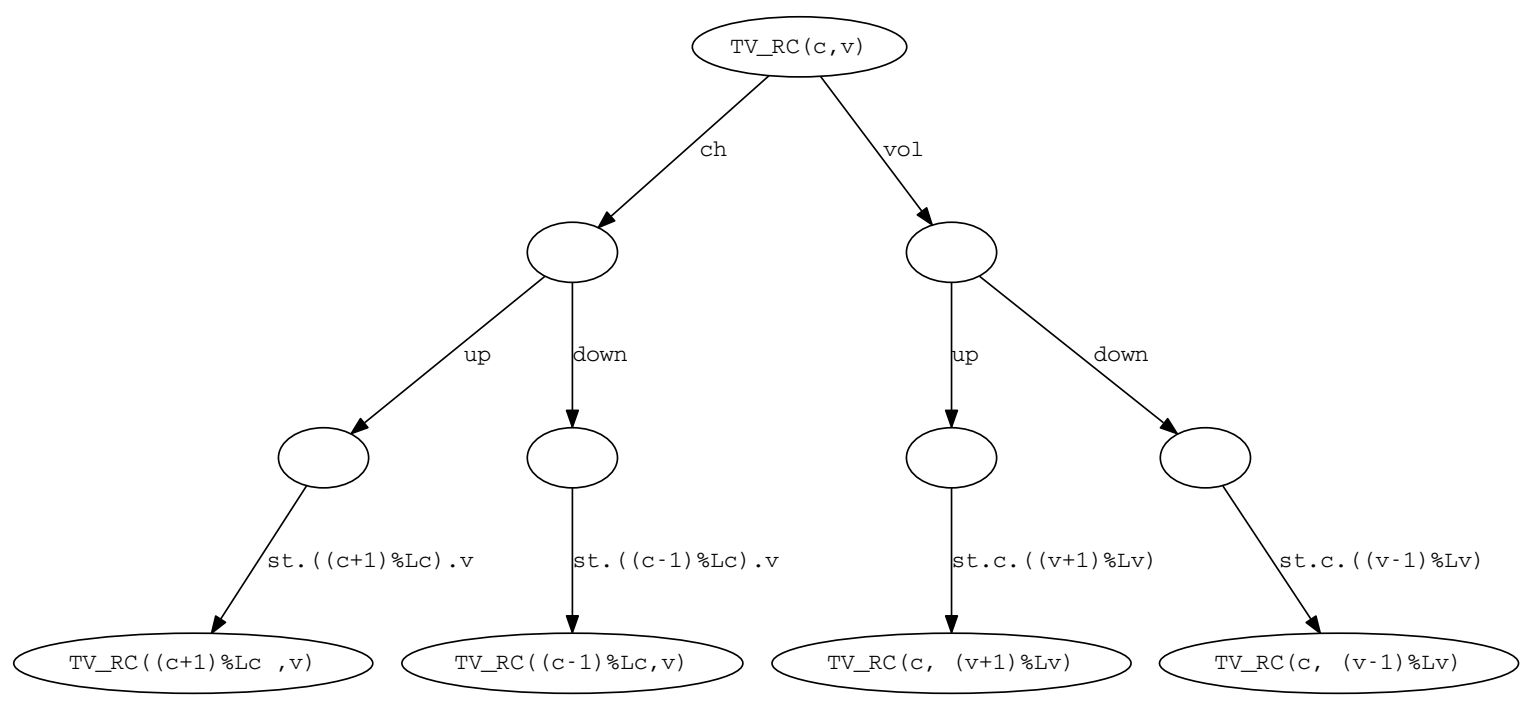

Source: José Dihego

and 2.8, respectively. The process $T V \_R C(C, V)$ represents a state where channel $\mathrm{C}$ and volume $\mathrm{V}$ are the last values sent to the TV. The user provides the input event vol to increase (up) or decrease (down) the TV volume or ch to navigate forward (up) or backward (down) on TV channels. Status output events (st) acknowledge the user of its commands.

The process TV_RC' $(\mathrm{C}, \mathrm{V}, \mathrm{b}, \mathrm{cn})$ in Figure 2.8 extends $T V \_R C(\mathrm{C}, \mathrm{V})$ by adding the new input event sett: it opens the TV settings menu, in which the user can adjust brightness (brig) or contrast (cont), whose values are registered by the state variables b and $\mathrm{cn}$, respectively. After providing brig or cont as input, it can use the regular input events up and down to make the image adjustments, which are echoed by output events through the channel st' .

If we recognise $T V \_R C^{\prime}(c, V, b, c n)$ as a proper extension to TV_RC $(c, V)$ we must decide which relation captures this kind of extension. Such a relation must allow new-incontext input events (events not allowed by the original specification at that point), as sett, followed by a finite number of new-in-context input/output events, as brig, up, and $\left\{\left|s t^{\prime}\right|\right\}$, provided the extension converges (it offers what was expected before the new-in-context event happened) to the original behaviour, as TV_RC' $(c, V,((b+1) \circ L b), c n)$ does after the trace 〈sett,brig, up〉: it offers $c h$ and vol, with the sate variables $c$ and $v$ unchanged by what happened after sett. In summary, Granpa can share the new TV remote control with Boy, without being stuck with the new features.

One can recognise the CSP failures refinement (Definition 2.2) as a candidate to capture the relationship between TV_RC and TV_RC', provided the new events $\{\mid$ sett, brig, st', cont $\mid\}$ are hidden in the extension, but FDR3 (the standard CSP model checker) quickly shows this is not the case as the following assertion fails:

assert $T V \_R C(c, v) \quad\left[F=T V_{-} R C^{\prime}(c, v, b, c n) \backslash\{\mid\right.$ sett, brig, cont, st' $\mid\}$ 


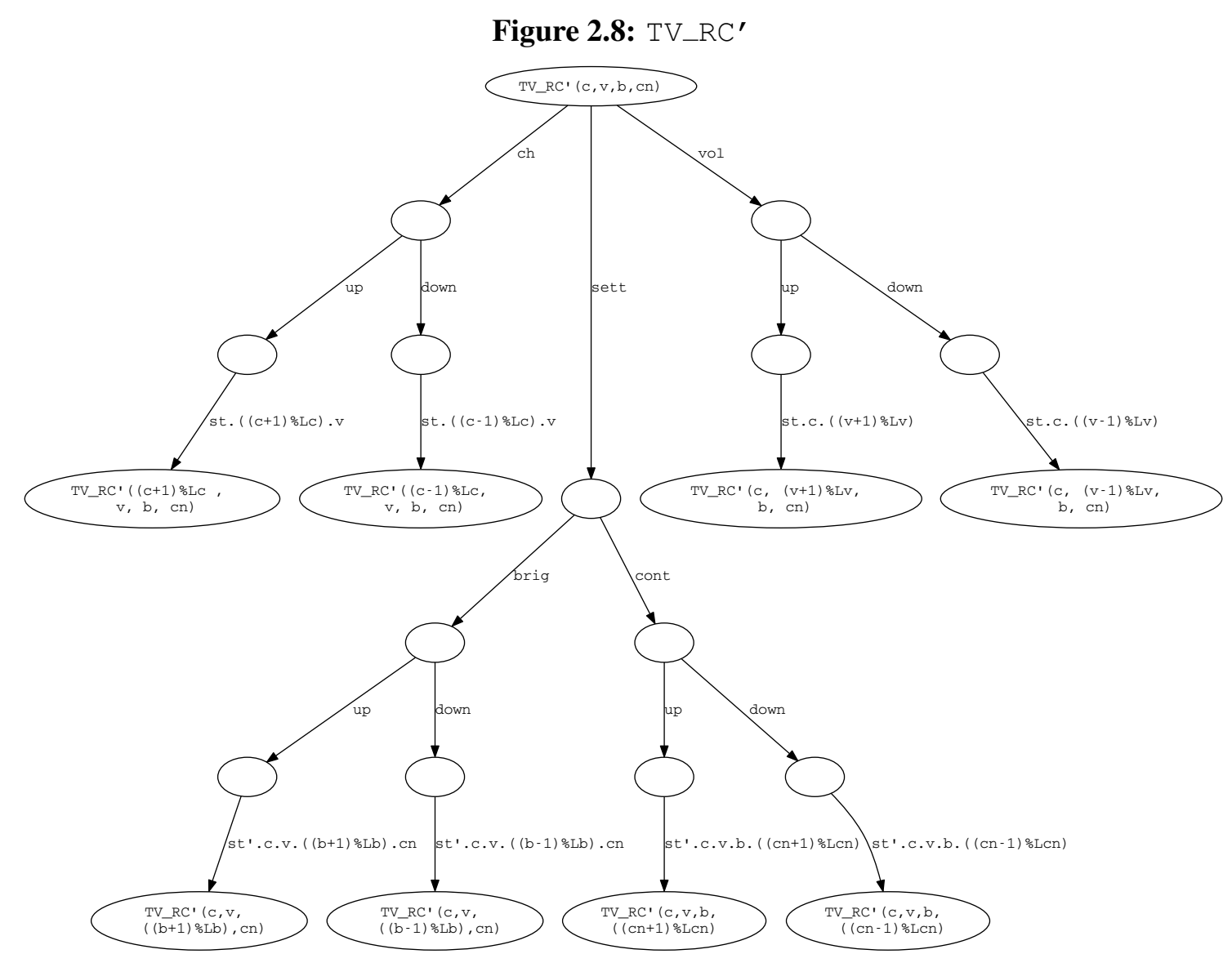

Source: José Dihego

This happens because the events up and down are used in new contexts in TV_RC' to adjust brightness and contrast. This kind of relationship in which we use existing events with a different meaning (in new contexts) cannot be captured by failures refinement.

The work of (WEHRHEIM, 2003) defines four subtyping relations for behaviour specifications, which allow functionality extension. The first issue with these candidate relations is that they do not differentiate inputs from outputs (as we discuss in the next section) and, moreover, extensions can only be defined in terms of new events, which can be concealed (they may not be communicated), hidden (makes them internal), explained (in terms of existing events) or restricted (completely forbids them). None of these four relations, as failures refinement modulo hiding, can properly capture the intended relationship between TV_RC and TV_RC' .

The ioco (input-output conformance) relation (BIJL; RENSINK; TRETMANS, 2004) allows extensions to admit new inputs (more functionalities) and to restrict outputs (more determinisms), but it does not obligate extensions, after a new input, to adhere (converge) to the original behaviour. Taking our example into account, ioco would admit TV_RC' inputs sett and then behaves as STOP (or anything else, including a divergent process). Therefore, the user Boy would not be able to navigate on the TV channel list, if he tried to adjust the image settings.

This discussion highlights the fact that the current behaviour relations cannot cope with functionality extension in a scenario where we need to add new events, to use existing events 
with different meanings (in different contexts) and to distinguish input from output events. These are the main motivations for convergence, which we formalise and discuss in depth in Chapter 4.

\section{The BRIC component model}

Component-based model driven development (SZYPERSKI, 1998) is a well recognised approach to develop complex systems and has been successfully applied in industry. Its basic idea is that complex systems can be built from simpler ones, called components, with well-defined interface and behaviour. The BRJC component model (RAMOS, 2011) (RAMOS; SAMPAIO; MOTA, 2009) formalises the core Component-Based Model Driven Development concepts (SZYPERSKI, 1998): components, interfaces, channels and behaviour. Moreover it supports component compositions, where deadlock freedom is ensured by construction. Component refinement and inheritance relations, although an important part of a component model, were not addressed in $\mathcal{B R J C}$ and they constitute the main motivation for this work.

In the $\mathcal{B R J C}$ model, components are reactive entities that can interact via communication channels. The behaviour of these components are represented by a subclass of CSP named I/O processes. This kind of processes distinguish input from output and, moreover, obey some behavioural patterns, which we will meet soon. Components interact asynchronously (via buffered channels) without deadlock. This is guaranteed by construction and is driven by a set of composition rules. Components are the building blocks of $\mathcal{B R J C}$ and are formally defined by contracts:

Definition 2.3 (Component contract). A component contract $C t r$ comprises an observational behaviour $\mathcal{B}$, a set of communication channels $\mathcal{C}$, a set of interfaces $\mathcal{J}$ (data types), and a total function $\mathcal{R}: \mathcal{C} \rightarrow \mathcal{J}$ between channels and interfaces of the contract:

$$
C t r:\langle\mathcal{B}, \mathcal{R}, \mathcal{J}, \mathcal{C}\rangle
$$

A communication channel $c \in \mathcal{C}$ is just a CSP channel with a declared type, which is some $i \in \mathcal{J}$. This implies necessarily that $c=\mathcal{R}(i)$. In CSP terms it is equivalent to the following declaration "channel $c: i$ ", where $i$ is a non-empty set. Since $\mathcal{R}$ is a total function between $\mathcal{C}$ and $\mathcal{J}$ we have that $\operatorname{dom} \mathcal{R}=\mathcal{C}$ and $\operatorname{ran} \mathcal{R}=\mathcal{J}$. The behaviour $\mathcal{B}$ is an $\mathrm{I} / \mathrm{O}$ process, a subclass of CSP processes defined as follows.

Definition 2.4 (I/O process). A CSP process is an I/O process if and only if the following conditions hold:

- I/O Channels. Every channel in $P$ is an I/O channel. A channel $c$ is an I/O channel if there exists two functions, inputs $(c, P)$ and outputs $(c, P)$ such that:

- inputs $(c, P) \cup$ outputs $(c, P)=\{|c|\}$, and

- $\operatorname{inputs}(c, P) \cap$ outputs $(c, P)=\emptyset$

where $\{|c|\}$ stands for the set of events communicated through $c$. 
This guarantees that every communication of $P$ through the channel $c$ is either an input or an output event and their respective sets are disjoint.

- Infinite Traces. $P$ has an infinite set of traces, but a finite state-space. It means components offer a finite set of services (events), but offers them continuously.

- Divergence-freedom. $P$ is divergence-free. Since it has a finite state-space, this guarantees that there is no $\tau$ loop in $P$ 's LTS. It means non-determinism is bound in $P$ : given the set of events communicated by $P$ we can always deduce a finite set of acceptances (or conversely refusals sets), which is crucial when outputs from one process are consumed by the other in deadlock-free compositions.

- Input Determinism. If a set of input events in $P$ is offered to the environment, none of them are refused. Formally, we say a process $P$ is input deterministic if:

$$
\forall s^{\wedge}\langle c . a\rangle: \mathcal{T}(P) \mid c . a \in \operatorname{inputs}(c, P) \bullet(s,\{c . a\}) \notin \mathcal{F}(P)
$$

A deterministic process is one where every state has no more than one $\tau$ leading out of it. This implies that if there is a choice, then it is an external one. An input deterministic process is one that inputs deterministically: if a set of inputs are offered to the environment, this can choose any of them. The control about process inputs relies on its environment.

- Strong Output Decisive. All choices (if any) among output events on a given channel in $P$ are internal. The process, however, must offer at least one output on that channel. Formally, we say a process is strong output decisive if:

$$
\begin{array}{r}
\forall s^{\wedge}\langle c . b\rangle: \mathcal{T}(P) \mid c . b \in \text { outputs }(c, P) \bullet(s, \text { outputs }(c, P)) \notin \mathcal{F}(P) \wedge \\
(s, \text { outputs }(c, P) \backslash\{c . b\}) \in \mathcal{F}(P)
\end{array}
$$

This behavioural property can be understood as the dual of input determinism. While the control over inputs resides in the hands of the process environment, the control about what it outputs is its solely responsibility. If $P$ reaches a state where some output is possible, the environment can only know that the next event is necessarily an output, although it does not know which one.

The use of recursion, in I/O processes, gives some of the expressiviness of contextfree grammars (CHOMSKY, 1965), but not all HOARE (1978). I/O process can only define those languages that can be parsed from left to right without backtracking or look-ahead (it requires left-to-right parsability without back-tracking). However, the use of parallelism by the I/O process language permits definition of languages which are not context-free. 


\section{Communication protocols}

The behaviour of a component $T$ is given in terms of an I/O process (say, $\mathcal{B}$ ). It can be accessed by other components through its communication points or I/O channels $\mathcal{C}$. It is worth mentioning that $\mathcal{C}$ does not necessarily comprises all channels used in the definition of $\mathcal{B}$, which allows $T$ to abstract/hide some implementation details from its environment (other components). From the point of view of a component being connected to $T$, through a channel $c \in \mathrm{e}$, the portion of the $T$ behaviour that matters is that exhibited through $c$. In fact, the four $\mathcal{B R J C}$ composition rules analyse such behavioural projections prior to connect components, aiming that the resulting component to be deadlock-free by construction. A communication protocol is a deadlock-free $\mathrm{I} / \mathrm{O}$ process whose communicated events are comprised of a unique channel.

Definition 2.5 (Communication protocol). We say that an $\mathrm{I} / \mathrm{O}$ process $P$ is a communication protocol if and only if:

$P$ is deadlock free and exists an I/O channel $c$ such that: $\operatorname{inputs}(P) \subseteq\{|c|\} \wedge$ outputs $(P) \subseteq\{|c|\}$

Therefore, by Definition 2.5 if $\mathcal{B}$ is deadlock-free we have that for every $c \in \mathcal{C}$, the process $\mathcal{B} \backslash(\Sigma \backslash\{c\})$, which stands for the process $\mathcal{B}$ hiding all events in $\Sigma$, except $c$, is a communication protocol. The recurrence of this construction in BRJE (RAMOS, 2011) has motivated the following definition.

Definition 2.6 (Projection). If $P$ is an I/O process and $c$ is a $I / O$ channel, we define the projection of $P$ over $c, P \nmid c$ or $\operatorname{Prot}_{I M P}(P, c)$, as follows:

$$
P \nmid c=P \backslash(\Sigma \backslash\{|c|\})
$$

By the semantics of hiding (Section 2.1), the process $P \backslash(\Sigma \backslash\{|c|\})$ behaves as $P$, where all events in the alphabet $\Sigma$, except those communicated through the channel $c$, are hidden (renamed to the invisible event $\tau$ ). Communication protocols are used in the $\mathcal{B R J \mathcal { C }}$ component model to study the behaviour a component exhibits through a specific channel in its interface. The composition rules connect invariably a pair of channels. Therefore they are concerned with the compatibility, in different degrees, between communication protocols exhibited by these channels being connected. Since outputs from one channel will be consumed as inputs by the other one, and vice versa, the first level of compatibility, given by Definition 2.7, ensures that all outputs from a communication protocol $P$ can, potentially, be consumed by $Q$ (outputs $(P) \subseteq \operatorname{inputs}(Q)$ ) and vice versa (outputs $(Q) \subseteq$ inputs $(P)$ ). Moreover, $P$ and $Q$ communicate through different channels, i.e., they have different alphabets. In the introduction of the CSP parallel operator we explained that two processes synchronise on an event if they both can offer such an event at the same time; therefore, by having different alphabets it is impossible to stablish the required synchronisation directly. This is possible thanks to renaming operations (embedded in the $\mathcal{B R J C}$ 
composition rules) that maps inputs of $P$ to outputs of $Q$, and vice versa. In fact when we check if outputs $(P) \subseteq$ inputs $(Q)$, rigorously, we are checking if $R^{*}($ outputs $(P)) \subseteq$ inputs $(Q)$ or equivalently whether out puts $(P) \subseteq R^{-1^{*}}$ (inputs $(Q)$ ), where $R$ maps the $P$ 's outputs to the $Q$ 's inputs and $R^{*}$ is the transitive closure of the relation $R$. We discuss further about this when introducing the composition rules.

Definition 2.7 (Conjugate protocols). Let $P$ and $Q$ be two communication protocols. $P$ and $Q$ are conjugate (denoted by $P \sim Q$ ) if, and only if:

$$
\begin{array}{r}
\text { outputs }(P) \subseteq \operatorname{inputs}(Q) \wedge \text { outputs }(Q) \subseteq \operatorname{inputs}(P) \\
\text { outputs }(P) \cap \text { outputs }(Q)=\emptyset \wedge \text { inputs }(P) \cap \operatorname{inputs}(Q)=\emptyset
\end{array}
$$

If two communication protocols are not conjugate, then composing them by hooking their channels results in deadlock, because one can output something that cannot be input by the other in the composition, which leads to an ungranted request, a well know source of deadlock ROSCOE (2005). It happens because a protocol can, inevitably, outputs an event not recognised by the other, which generates an ungranted request, a recognised source of deadlock (ROSCOE; DATHI, 1987). The fact is that, if they are conjugate, we have a syntactic guarantee that they might be composed without deadlock. This fact is indeed guaranteed by the next definition, protocol compatibility: two protocols are compatible if they are deadlock-free, conjugate and, being in a state where both have done a common trace, at least one of them is able to output and the other able to consume (synchronise on) any possible output. Therefore, every request (an output) is granted (consumed by an input). Note that there is always an output being generated, by one process or another, so there is not the possibility that both stuck waiting for outputs from each other.

Definition 2.8 (Protocol compatibility). Let $P$ and $Q$ be two deadlock-free communication protocols, such that $P$ and $Q$ are conjugate. The protocols $P$ and $Q$ are compatible $(P \approx Q)$ if, and only if:

$$
\begin{aligned}
& \forall s: \operatorname{traces}(P) \cap \operatorname{traces}(Q) \bullet(\operatorname{out}(P, s) \neq \emptyset \wedge \operatorname{out}(P, s) \subseteq \operatorname{in}(Q, s)) \vee \\
&(\operatorname{out}(Q, s) \neq \emptyset \wedge \operatorname{out}(Q, s) \subseteq \operatorname{in}(P, s))
\end{aligned}
$$

If both processes can output, then we have by Definition 2.8 that outputs can be leaked, not consumed by (not synchronised with) input events. It motivates the next definition for compatibility, strong protocol compatibility; as the name suggests, it is the most restrictive relation between protocols and guarantees, besides the absence of ungranted requests, that all information generated (outputs) by one process is consumed by the other. 
Definition 2.9 (Strong protocol compatibility). Let $P$ and $Q$ be two deadlock-free communication protocols, such that $P$ and $Q$ are conjugate. The protocols $P$ and $Q$ are strong compatible (denoted $P \approx Q$ ) if, and only if:

$$
\begin{aligned}
\forall s: \operatorname{traces}(P) \cap \operatorname{traces}(Q) & \bullet(\operatorname{out}(P, s) \neq \emptyset \vee \operatorname{out}(Q, s) \neq \emptyset) \wedge \\
& \operatorname{out}(P, s) \subseteq \operatorname{in}(Q, s) \wedge \operatorname{out}(Q, s) \subseteq \operatorname{in}(P, s)
\end{aligned}
$$

It is simple to deduce that $P \approx Q \Rightarrow P \approx Q \Rightarrow P \sim Q$, meaning that strong compatibility implies in compatibility, which itself implies that the protocols are conjugate.

\section{Asynchronous communication}

The CSP processes interact with each other synchronously. Therefore if two processes synchronise on an event, it only happens if both processes engage simultaneously on it. Clearly this communication model is not appropriate if we want to connect channels of the same component, because generally it cannot agree on two events at the same time. As an intuition, consider an I/O process $P$ that outputs $c$.out and inputs $d$.in sequentially ( $c$ and $d$ are channels); by the CSP semantics, there is no way to express the synchronisation between these events because they are not communicated simultaneously. A workaround is to use a buffer to hold c.out until d.in is ready to be offered, simulating an asynchronous communication medium, which is roughly the solution adopted in $\mathcal{B R J C}$ to allow internal connections between components. The fact that $\mathcal{B R J C}$ adopts an asynchronous communication model simulated by buffered communication is supported by Roscoe's work on buffer tolerance for CSP (ROSCOE, 2005), which allows us to infer results about buffered systems from unbuffered analogues. A buffer can hold events from a process and forward them to another (or itself), which emulates an asynchronous communication medium between them, while the whole combination (processes and buffers) communicates synchronously.

In order to be able to express the properties of a buffer in $\mathcal{B R J \mathcal { C }}$, we introduce the trace notation $\downarrow$ from (ROSCOE, 1998): if $t \in \Sigma^{*}$ and $e v \in \Sigma$, then $t \downarrow e v$ gives the number of times the event $e v$ appears on the trace $t$. If a CSP process $B$ is a buffer in $\mathcal{B R J C}$, it has one input (left) and one output (right) channel, both of which have the same type and the following holds:

- $\operatorname{divergences}(B)=\emptyset: B$ is divergent free.

- $s \in \operatorname{traces}(B) \Rightarrow s \in(\{\mid \text { left } \mid\} \cup\{\mid \text { right } \mid\})^{*} \wedge s \downarrow$ right $\leq s \downarrow$ left $: B$ inputs on left and outputs on right. It correctly copies all its inputs to its output channel, without loss or reordering.

- $(s, X) \in$ failures $(B) \wedge s \downarrow$ right $=s \downarrow$ left $\Rightarrow X \cap\{\mid$ left $\mid\}=\emptyset$ : whenever it is empty (i.e., it has output everything it has input) then it must accept any input. 
- $(s, X) \in$ failures $(B) \wedge s \downarrow$ right $<s \downarrow$ left $\Rightarrow\{\mid$ right $\mid\} \nsubseteq X$ : whenever it is non-empty, then it cannot refuse to output.

When a pair of channels are connected by a $\mathcal{B} \mathcal{R J}$ composition rule, outputs from one will be inputs for the other and vice versa. There are two directional flows of communication. Therefore, two buffers are required to emulate an asynchronous medium between these channels, one for each flow. A problem arises at this point because a CSP buffer requires input and outputs values to have the same type. As an event's type encompasses the name of the channel through which it is communicated, we need to change the classical CSP buffer to receive outputs from one channel and convert them into inputs to the other, in both directions of the communication. It is achieved by embedding into $B$ a mapping function $R$, where $B_{s}^{n}(R)$ denotes a $n$-size buffer holding a sequence of events $s$; it inputs and outputs events from $\operatorname{dom} R$ and $\operatorname{ran} R$, respectively, as follows:

$$
\begin{aligned}
B^{n}(R)= & B_{\langle\rangle}^{n}(R)=? x: \operatorname{dom} R \rightarrow B_{\langle x\rangle}^{n} \\
B_{s}^{n}(R)= & \# s<n \& ? x: \operatorname{dom} R \rightarrow B_{s^{\wedge}}^{n}\langle x\rangle \\
& \square R(\text { head }(s)) \rightarrow B_{\text {tail }(s)}^{n}(R)
\end{aligned}
$$

This $n$-size buffer can bufferise up to $n$ elements. If it is empty it can only input, if it is full it can only output, otherwise it can do both. It inputs from $\operatorname{dom} R$ and outputs from $\operatorname{ran} R$. In theory, we can have $n \rightarrow \infty$ and $\#_{s}<n$ is always satisfied, but Roscoe has demonstrated that asynchronous communication can be emulated by a sufficient large $n$ (ROSCOE, 2005), if some behavioural properties, presented briefly, are met by the processes being connected. As we have said, the asynchronous connection of two channels must deal with two directional communication flows between them. For each flow we need a buffer parametrised by an appropriate mapping function, let us say $B^{n}(L)$ and $B^{n}(R)$; as each of these flows proceeds independently, the interleave of these buffers, $B^{n}(L) \| B^{n}(R)$, creates an asynchronous communication medium for these channels.

$$
B U F F_{I O}^{n}(L, R)=B^{n}(L) \| B^{n}(R)
$$

The buffer $B U F F_{I O}^{n}(L, R)$ can bufferise up to $n$ elements in each flow of communication. It can hold an unbounded number of elements, in each direction, if $n \rightarrow \infty$. It is the default buffer used by $\mathcal{B R J C}$ composition rules, where we assume a sufficient large $n$ (in the majority cases $n=1)$ and $L$ and $R$ functions that map outputs from one channel into inputs for another and vice versa.

Not all CSP specifications can have their communications intermediated by buffers. Roscoe's work on buffer tolerance for CSP specifies some behavioural properties that a specification must satisfy to be buffer tolerant: buffers of arbitrary or varying length may be introduced 
onto their channels without introducing any extra errors. The great advantage of this theory is that we can verify a specification without buffers and guarantee the same results for its bufferedanalogue (buffer tolerance ROSCOE (2005)), with a great reduction in the time/space check, thanks to the reduction of the specification state-space.

Buffer tolerance can be defined in different degrees depending on what we call extra errors; in $\mathcal{B R J \mathcal { C }}$ an extra error can be understood as a deadlock. In (ROSCOE, 2005), a CSP process is buffer tolerant with respect to deadlock freedom if it is confluent: whenever a state has two alternative events, then performing either of them does not preclude the other and furthermore performing them in either order leads to equivalent states.

The work of (RAMOS, 2011) lifts this concept to $\mathcal{B} \mathcal{R J C}$ to cope with the different nature of input/output events. An I/O process is I/O confluent if, whenever a state has two alternative events $e_{1}$ and $e_{2}$, then performing either of them does not preclude the other, unless it is a choice among inputs or outputs of the same channel.

Definition 2.10 (I/O confluence). Let $P$ be an $I / O$ process. Then $P$ is $I / O$ confluent if and only if:

$$
\begin{aligned}
\forall s^{\wedge} & \left\langle c_{1} . a\right\rangle^{\wedge} t, s^{\wedge}\left\langle c_{2} . b\right\rangle: \text { traces }(P) \mid c_{1} . a \neq c_{2} . b \bullet \\
& \left(c_{1} . a \in \text { inputs }(P) \wedge \exists i: \text { inputs }\left(P, c_{1}\right) \mid s^{\wedge}\left\langle c_{2} \cdot b, i\right\rangle \wedge\left(t-\left\langle c_{2} . b\right\rangle\right) \in \operatorname{traces}(P)\right) \vee \\
& \left(c_{1} . a \in \text { outputs }(P) \wedge \exists o: \text { outputs }\left(P, c_{1}\right) \mid s^{\wedge}\left\langle c_{2} . b, o\right\rangle^{\wedge}\left(t-\left\langle c_{2} . b\right\rangle\right) \in \operatorname{traces}(P)\right) \vee \\
& \left(c_{1}=c_{2} \wedge\left(\left\{c_{1} . a, c_{2} . b\right\} \subseteq \text { outputs }(P) \vee\left\{c_{1} . a, c_{2} . b\right\} \subseteq \text { inputs }(P)\right)\right)
\end{aligned}
$$

In the above definition if $t$ and $s$ are traces, then $t-s$ is $t$ with elements from $s$ deleted according to multiplicity, earliest first. Thus, $\langle a, a, b, c, d, a\rangle-\langle a, c, a\rangle=\langle b, d, a\rangle$, where $\{a, b, c, d\} \subseteq \Sigma$. If $c$ is a channel of $P$, then inputs $(P, c)$ and outputs $(P, c)$ stand for the inputs and outputs $P$ can do through $c$, respectively. Consider that there is a choice between $\left\{c_{1} . a, c_{2} . b\right\} \subseteq \operatorname{inputs}(P, c)$, then if $s^{\wedge}\left\langle c_{1} . a\right\rangle^{\wedge} t$ and $s^{\wedge}\left\langle c_{2} . b\right\rangle$ are traces of $P$ this definition guarantees that $s^{\wedge}\left\langle c_{2} . b, i\right\rangle^{\wedge}\left(t-\left\langle c_{2} . b\right\rangle\right)$ is also a trace of $P$, where the event $i$ can be any input offered through $c_{1}$. It means that $c_{2} . b$ can happen before $c_{1} . a$ (or any input event $i$ offered through $c_{1}$ ) and it might not appear after $\left(t-\left\langle c_{2} . b\right\rangle\right)$, so by communicating $c_{2} . b$ after or before $i$ does not affect any decision about the future state $P$ can reach after that. The same reasoning applies to $\left\{c_{1} . a, c_{2} . b\right\} \subseteq$ out puts $(P, c)$. If $c_{1}=c_{2}$ then an event can preclude another, but $P$ remains buffer tolerant with respect to deadlock freedom as proved by (RAMOS, 2011).

As a specification evolves towards computational feasibility in time and space, buffer tolerance means bounded buffer tolerance, i.e., buffers have a limit (ROSCOE, 2005). If a process can communicate an infinite unbroken sequence of outputs without performing an input, a bounded buffer can overflow refusing to hold any further outputs (although I/O processes have finite LTSs they can communicate an unbroken sequence of outputs by recursion). It is an undesirable situation, which is avoided if the specification processes satisfy the finite output property (FOP): 
Definition 2.11 (Finite output property). Let $P$ be an I/O process, and $C$ the set of channels used in $P$. We say that $P$ satisfies the finite output property (FOP) if, and only if, for all $c \in C$ the process $P \backslash$ outputs $(P, c)$ is divergence-free.

I/O processes are divergence-free; the absence of divergence after hiding the outputs in the original protocol guarantees this property. Since hiding events are renamed to $\tau \mathrm{s}$, the absence of divergence means that there is not an unbroken sequence of outputs performed by $P$ through each $c \in C$.

The $\mathcal{B R J C}$ composition rules support the hook of channels in a same component. There must be some sort of inner compatibility between these channels, specially concerning mutual interference. The most basic, but restrictive, guarantee against interference can be achieved if we assume the channels being connected are decoupled: the behaviour projection over them are equivalent to the interleaving of each one's projection.

Definition 2.12 (Decoupled channels). Let $P$ be an $I / O$ process and $\{c, z\}$ two $I / O$ channels. Then, $c$ and $z$ are decoupled in $P$ if, and only, if:

$$
P \nmid\{c, z\} \equiv_{\mathrm{F}}(P \uparrow c) \mid \|(P \uparrow z)
$$

The process $P$ behaves on $c$ independently from its behaviour on $z$. It has the same effect over buffer tolerance (concerning deadlock freedom) as if we were connecting channels from different components. A less restrictive, but more elaborated, form of compatibility is buffering self-injection. It guarantees that we can put a one-place buffer to intermediate the asynchronous communication between a component with itself, by connecting a pair of its channels, without introducing deadlock.

Definition 2.13 (Buffering self-injection compatibility). Let $P$ be a deadlock-free I/O process, and $c$ and $z$ two of its channels. Then $P \nmid\{|c, z|\}$ is buffering self-injection compatible if, and only if:

$$
\begin{aligned}
& \forall(s, X): \text { failures }(P \nmid\{|c, z|\}) \mid \\
& \left(s \downarrow O_{c}=s \downarrow I_{z}\right) \wedge\left(s \downarrow O_{z}=s \downarrow I_{c}\right) \bullet X \cap\left(O_{c} \cup O_{z}\right)=\emptyset \vee \\
& s \downarrow O_{c}>s \downarrow I_{z} \bullet(s \uparrow z, X \cup\{|c|\}) \in \text { failures }(P \nmid z) \vee \\
& s \downarrow O_{z}>s \downarrow I_{c} \bullet(s \uparrow c, X \cup\{|z|\}) \in \text { failures }(P \uparrow c) \\
& \text { where } O_{c}=\text { outputs }(P, c), O_{z}=\text { outputs }(P, z), I_{c}=\operatorname{inputs}(P, c) \text { and } I_{z}=\operatorname{inputs}(P, z)
\end{aligned}
$$

By Definition 2.13 an I/O process $P\{\{|c, z|\}$ is buffering self-injection compatible if whenever it has communicated the same number of outputs by $c$ and $z$ (two of its channels), it cannot refuse them to output, $X \cap\left(O_{c} \cup O_{z}\right)=\emptyset$. Otherwise more outputs have been communicated through $c\left(s \downarrow O_{c}>s \downarrow I_{z}\right)$ or through $z\left(s \downarrow O_{z}>s \downarrow I_{c}\right)$. If the former is true then no more 
Figure 2.9: Composition rules
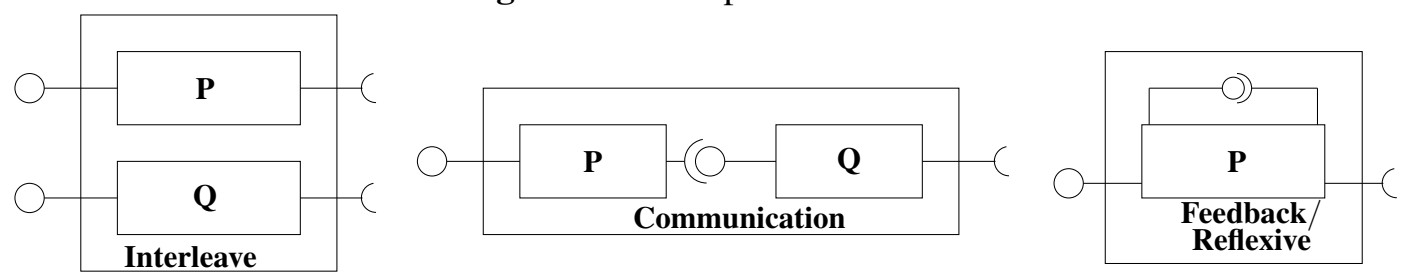

Source: José Dihego (based on (RAMOS, 2011))

outputs coming from $c$ can be accepted: $s \nmid z, X \cup\{|c|\}) \in$ failures $(P \nmid z)$; but if the latter is true it refuses to accept outputs from $z:(s \uparrow c, X \cup\{|z|\}) \in \operatorname{failures}(P \uparrow c)$.

Now we have the background to present the $\mathcal{B R J C}$ composition rules. They support trustworthy specification/development of systems made of components.

\section{Composition rules}

$\mathcal{B R J C}$ has four composition rules (asynchronous pairwise compositions mediated by buffers), namely: interleaving, communication, feedback, and reflexive. Each of these rules impose different side conditions, which must be satisfied by the involved components in order to guarantee deadlock freedom by construction. Using the rules, developers may synchronise pairs of channels of two different components, or even of the same component. The rules are illustrated in Figure 2.9. The result of a composition, as expected, is a component.

The $\mathcal{B R J C}$ rules rely on two basic forms of composition: asynchronous binary and unary compositions. By default, CSP processes communicate synchronously, and therefore to implement an asynchronous communication model in $\mathcal{B R J \mathcal { C }}$, the connections between components are mediated by finite buffers. As we explain in the sequel, the $\mathcal{B} \mathcal{R J C}$ rules act by composing channels pairwisely (possibly on the same component) and sequentially (two compositions cannot be made at the same time), which guarantees, by local analysis, behavioural properties preservation (such as deadlock freedom). Despite how complex are the connections established between components and the data flow they create, global properties are ensured by local analysis, and, moreover, the state-space of a composition mediated by a $n$-size buffer increases linearly on $n$ concerning the bufferless composition (which is not allowed on $\mathcal{B R J C}$ ). As these basic forms of composition do not impose any behavioural or structural restrictions, the resulting component can deadlock.

Definition 2.14 (Asynchronous binary composition). Let $P$ and $Q$ be two distinct component contracts, and $c \in \mathcal{C}_{P}$ and $z \in \mathcal{C}_{Q}$ two channels, such that $\mathcal{C}_{P}$ and $\mathcal{C}_{Q}$ are disjoint. Then, the asynchronous binary composition of $P$ and $Q, P\langle c\rangle=\langle z\rangle$, is given by:

$$
P_{\langle c\rangle} \asymp\langle z\rangle=\left\langle\mathcal{B}_{P}\left\|_{\{|c|\}} B U F F_{I O}^{n}\left(R_{I O}^{c \rightarrow z}, R_{I O}^{z \rightarrow c}\right)\right\|_{\{|z|\}} \mathcal{B}_{Q}, \mathcal{R}^{\prime}, \mathcal{J}^{\prime}, \mathrm{C}^{\prime}\right\rangle
$$




$$
\text { where } \begin{aligned}
\mathcal{C}^{\prime} & =\left(\mathcal{C}_{P} \cup \mathcal{C}_{Q}\right) \backslash\{c, z\}, \mathcal{R}^{\prime}=\mathcal{C}^{\prime} \triangleleft\left(\mathcal{R}_{P} \cup \mathcal{R}_{Q}\right), \\
\mathcal{J}^{\prime} & =\operatorname{ran} \mathcal{R}^{\prime} \text { and } R_{I O}^{a \rightarrow b}=\{\text { a.out.x } \mapsto \text { b.in.x }\} .
\end{aligned}
$$

The asynchronous binary composition (Definition 2.14) hooks two components, say $P$ and $Q$, with disjoint communication points, by their respective channels $c$ and $z$. Instead of communicating directly, their communications are buffered by $B U F F_{I O}^{n}\left(R_{I O}^{c \rightarrow z}, R_{I O}^{z \rightarrow c}\right)$. Note that this buffer is parametrised by a map of the form $R_{I O}^{a \rightarrow b}=\{$ a.out. $x \mapsto$ b.in. $x\}$ : it maps output events that can be communicated through $a$ to the respective input events communicated through $b$. Remember that $B U F F_{I O}^{n}$ must mediate two directional flows: outputs from $c$ to $z\left(R_{I O}^{c \rightarrow z}\right)$ and vice versa $\left(R_{I O}^{z \rightarrow c}\right)$. Once a channel is connected, it cannot be used by any other connection, so it must be removed from the resulting component interface, $\left(\mathcal{C}_{P} \cup \mathcal{C}_{Q}\right) \wedge\{c, z\}$, which obligates the respective adequacies of $\mathcal{J}$ and $\mathcal{R}$ of the resulting component.

The asynchronous unary composition (Definition 2.15) hooks two channels, say $c$ and $z$ of the same component $P$. It allows $P$ to send and receive information to/from itself. It can be very useful if $P$ has inner components, that must be connected (see, for example, the dining philosophers in $\mathcal{B R J C}$ (RAMOS, 2011)). The resulting component behaviour is that of $P$ synchronised with the buffer $B U F F_{I O}^{n}\left(R_{I O}^{c \rightarrow z}, R_{I O}^{z \rightarrow c}\right)$. It allows, for example, the composition to generate an output by $c$, which is buffered, releasing it to consume (synchronise) such output by an input from $z$. The same reasoning used in the binary composition holds in the definition of channels, interfaces and the relation between them in the resulting component.

Definition 2.15 (Asynchronous unary composition). Let $P$ be a component contract, and $\{c, z\} \subseteq$ $\mathcal{C}_{P}$ two of its channels. Then, the asynchronous unary composition of $P$ by hooking $c$ and $z$, $P \asymp \mid\langle\langle z\rangle$, is given by:

$$
P \asymp \mid \begin{gathered}
\langle c\rangle \\
\langle z\rangle
\end{gathered}=\left\langle\mathcal{B}_{P} \|_{\{|c, z|\}} B U F F_{I O}^{n}\left(R_{I O}^{c \rightarrow z}, R_{I O}^{z \rightarrow c}\right), \mathcal{R}^{\prime}, \mathrm{J}^{\prime}, \mathrm{C}^{\prime}\right\rangle
$$

where $\mathcal{C}^{\prime}=\mathcal{C}_{P} \backslash\{c, z\}, \mathcal{R}^{\prime}=\mathcal{C}^{\prime} \triangleleft \mathcal{R}_{P}$, and $\mathcal{J}^{\prime}=\operatorname{ran} \mathcal{R}^{\prime}$ and $R_{I O}^{a \rightarrow b}=\{$ a.out.$x \mapsto$ b.in.x $\}$.

The interleave composition rule is the simplest form of composition. It aggregates two independent components such that, after composition, these components still do not communicate between themselves. They directly communicate with the environment as before, with no interference from each other.

Definition 2.16 (Interleave composition). Let $P$ and $Q$ be two component contracts, such that $\mathcal{C}_{P} \cap \mathcal{C}_{Q}=\emptyset$.

Then, the interleave composition of $P$ and $Q$ (namely $P[\||| Q$ ) is given by:

$$
P[\|||] Q=P\langle\rangle \asymp\langle\rangle Q
$$


The resulting component, $P_{\langle\rangle} \asymp\langle\rangle$, , is given in terms of asynchronous binary composition, without any channel $(\langle\rangle)$ being connected. It implies that channels, interfaces and the relation between them in the composition are given by the union of those elements of $P$ and $Q$.

The communication composition represents the most common way for linking complementary channels of two different components. As interleaving, it is given in terms of asynchronous binary composition, but it connects channels from $P$ and $Q$ components. The assembled channels cannot be used in subsequent compositions, as imposed by Definition 2.14.

Definition 2.17 (Communication composition). Let $P$ and $Q$ be two component contracts, and ic and oc two communication channels, such that:

i. ic $\in \mathcal{C}_{P} \wedge$ oc $\in \mathcal{C}_{Q}$;

ii. $\mathcal{C}_{P} \cap \mathcal{C}_{Q}=\emptyset$, and;

iii. the port protocols $P \uparrow i c\left[\left[R_{I O}^{i c \rightarrow o c}\right]\right]$ and $Q \uparrow o c\left[\left[R_{I O}^{o c \rightarrow i c}\right]\right]$ are $I / O$ confluent strong compatible and satisfy the finite output property.

Then, the communication composition of $P$ and $Q$ (namely $P[i c \leftrightarrow o c] Q$ ) via ic and oc is defined as follows:

$$
P[i c \leftrightarrow o c] Q=P_{\langle i c\rangle} \asymp\left\langle{ }_{\langle o c\rangle} Q\right.
$$

The next two compositions allow the link of two complementary channels of a same component by means of asynchronous unary composition. The feedback composition provides the possibility of creating safe cycles for components with a tree topology. It achieves this by, among others conditions, ensuring that the channels being connected are decoupled.

Definition 2.18 (Feedback composition). Let $P$ be a component contract, and ic and oc two communication channels, such that:

i. the protocols $\operatorname{Prot}_{I M P}(P, i c)\left[\left[R_{I O}^{i c \rightarrow o c}\right]\right]$ and $\operatorname{Prot}_{I M P}(P, o c)\left[\left[R_{I O}^{o c \rightarrow i c}\right]\right]$ are I/O confluent strong compatible and satisfy the finite output property, and;

ii. $\{$ ic,$o c\} \subseteq \mathcal{C}_{P}$ and ic and oc are decoupled in $P$.

Then, the feedback composition $P(P[o c \hookrightarrow i c])$ hooking oc to ic is defined as follows:

$$
P[o c \hookrightarrow i c]=P \asymp \mid \begin{aligned}
& \langle i c\rangle \\
& \langle o c\rangle
\end{aligned}
$$

The last composition rule, reflexive, is more general than the feedback composition, since its required conditions are implied by those needed to connect components using feedback. It assumes the protocols exhibited through the channels being connected are self-injection 
compatible, which is weaker than assuming these channels are decoupled. However, it is also more costly regarding verification, since it is able to assemble dependent channels (feedback assembles only independent channels), and so in general it requires a global analysis to ensure deadlock freedom. On the other hand, reflexive composition allows to connect channels in a cyclic topology, whereas feedback is restricted to tree topologies.

Definition 2.19 (Reflexive composition). Let $P$ be a component contract, and ic and oc two communication channels, such that:

i. $\{i c, o c\} \subseteq \mathcal{C}_{P}$, and;

ii. $P \nmid\{i c, o c\}$ is buffering self-injection compatible and satisfies the finite output property.

Then, the reflexive composition $P$ (namely $P\left[\right.$ oc $^{-}$ic $]$) hooking oc to ic is defined as follows:

$$
P[i c \stackrel{-}{\hookrightarrow} o c]=P \asymp \mid \begin{aligned}
& \langle i c\rangle \\
& \langle o c\rangle
\end{aligned}
$$

Any system developed with the $\mathcal{B R J C}$ composition rules is deadlock-free. Theorem 2.1 is proved in (RAMOS, 2011).

Theorem 2.1 (Deadlock-free Component Systems). Any system $S$ in normal form, built from deadlock-free components, is deadlock-free.

$$
S::=P \quad|\quad S[|| \mid] S \quad| \quad S\left[c_{1} \leftrightarrow c_{2}\right] S \quad\left|\quad S\left[c_{1} \hookrightarrow c_{2}\right] \quad\right| \quad S\left[c_{1} \hookrightarrow c_{2}\right]
$$

These rules can express any parallel composition that can be made with component I/O processes in CSP and more, because they allow inner connections, which is not directly supported by the synchronous CSP communication model.

\section{Component inheritance and refinement}

Class inheritance, in the object-oriented paradigm, is a well-established concept (LISKOV; WING, 1994; WEGNER; ZDONIK, 1988; AMERICA, 1991). In these works, inheritance is driven by the substitutability principle: an instance of the subtype (subclass) can be used wherever an instance of the supertype (superclass) was expected, without any noticeable difference.

The substitutability principle has also driven the development of inheritance relations in the context of process algebras (CSP, CCS (MILNER, 1982), PAT (SUN; LIU; DONG, 2008)), and more concrete operational models like I/O labelled transition systems. Some of the most well known works about this topic (WEHRHEIM, 2003; FISCHER, 1997) have used the failures behavioural model of CSP to define process inheritance relations, which satisfy in different 
degrees, the substitutability principle. Following the same approach, but with a special focus on code reuse and algebraic equivalence, our previous work (DIHEGO; ANTONINO; SAMPAIO, 2013) has developed a set of algebraic laws for process subtyping in the OhCircus language (CAVALCANTI; SAMPAIO; WOODCOCK, 2005), an integration of Z (SPIVEY, 1989), CSP and object-oriented concepts.

Inheritance relations for process algebras cannot be dissociated from refinement. In fact, refinement is the strongest form of relationship between CSP processes that fulfils the substitutability principle (ROSCOE, 1998). Given the importance of CB-MDD, a formal approach for implementing and reasoning about components must have, in a development and evolutionary perspective, notions for component refinement and inheritance. This is where we concentrate our efforts by proposing definitions of component refinement and inheritance, and how they relate. Although we express our results in the $\mathcal{B R J C}$ component model, they can be applied in any approach for CB-MDD provided behaviour can be equivalently expressed as an I/O labelled transition system. Inputs are under environment's control and outputs are the sole responsibility of the component generating it. It represents a considerable class of systems, which encompasses many industrial client-server applications.

Component refinement is not a new concept, as we can see in rCOS (Refinement of Component and Object System) (LIU; JIFENG; LI, 2005), a CB-MDD approach, whose semantics is defined using Hoare and He's Unifying Theories of Programming (UTP). It supports interfaces, contracts, protocols, components, compositions, component publication and refinement. Although similar to $\mathcal{B} \mathcal{R J C}$, it does not differentiate inputs from outputs and does not provide trustworthy composition rules. The component model developed in (HENNICKER; JANISCH; KNAPP, 2010) specifies behaviour as I/O transition systems, and although it differentiates inputs from outputs, compositions and behavioural properties are not related.

The major contribution of our work concerns component inheritance. As far as the authors are aware, this is the first time component inheritance relations are developed for a formal and sound CB-MDD approach. We also relate it with component refinement, showing that every specification can, as an alternative, evolve via inheritance. This contribution relies on top of a novice concept called behavioural convergence: a relation between I/O labelled transition systems (I/O processes, a subclass of CSP processes) where a sub-process, by deciding to behave in its own way, is always able to converge to its parent's behaviour, as we will see in more detail in Chapter 4. Our relations satisfy the substitutability principle, which attains the general definition but has some adaptations for our component model, where behavioural properties are guaranteed by construction, with a special focus on deadlock freedom. 


\section{3 3}

\section{BRIC semantics and refinement}

According to the principle of type substitutability (LISKOV; WING, 1994), an instance of the subtype should be usable wherever an instance of the supertype is expected, without a client being able to tell the difference. The strongest relation that fulfils this principle is refinement (ROSCOE, 1998). In CSP, we have, for each denotational model, a refinement relation that obeys substitutability in different degrees; such a notion is absent in $\mathcal{B R J C}$. We contribute with a refinement relation for $\mathcal{B} \mathcal{R J \mathcal { C }}$ stated on a denotational semantic model. We also prove monotonicity of our relation with respect to the $\mathcal{B R J C}$ composition rules. This chapter is based on our results presented in (Oliveira, M. and Sampaio, A. and Antonino,P. and Dihego, J. and Filho, M. C. and Bryans, J., 2014).

\section{BRIC semantics}

In order to reason about the meaning of a program, a formal semantics is required. This can be given in the denotational, algebraical or operational style. Whatever we choose (driven by project restrictions or taste), the properties satisfied in one model must hold in the others as well (congruence of semantics). As we already mentioned, CSP has three main denotational models: traces $(\mathcal{T})$, stable failures $(\mathcal{F}$, or just failures) and failures-divergences $(\mathcal{N})$. Depending on the properties we are interested in, one model is more suitable or efficient (model checking time) than others. For example, if what a process can do (or refuse to do) at a certain point of this execution is relevant, it is preferable the adoption of the failures model $\mathcal{F}$, since it copes with refusals (and consequently with acceptances). The use of a denotational model in this work allows the reuse important results from CSP (ROSCOE, 1998), particularly those on the traces and failures denotational models.

We start by proposing a function $\mathcal{S} \llbracket \cdot \rrbracket$ from a $\mathcal{B} \mathcal{R J C}$ component to an underpinning mathematical model. Consider the component $T:\langle\mathcal{B}, \mathcal{R}, \mathcal{J}, \mathcal{C}\rangle$; the semantics of $T$ is given by $\mathcal{S} \llbracket T \rrbracket$, such that: 


$$
\mathcal{S} \llbracket T \rrbracket=(\text { failures }(\mathcal{B}),\{(c, \text { failures }(\mathcal{B} \nmid c)) \mid c \in \mathcal{C}\})
$$

Each declaration of channel $c$ has an interface $i \in \mathcal{J}$ (a type comprising a set of values and operations), and it implies that $c \mathcal{R} i$. From this point of view, although $\mathcal{R}$ and $\mathcal{J}$ do not appear explicitly in $\mathcal{S} \llbracket T \rrbracket$ they are implicitly there through $c$ 's declaration. This semantics captures the relevant properties of a $\mathcal{B R J C}$ component: its overall behaviour and those exhibited through its channels, which are crucial in composition rules. Note that $\mathcal{C}$ stands for a set of names and each pair $(c$,failures $(\mathcal{B} \uparrow c)$ ) (for all $c \in \mathcal{C}$ ) is constructed by retrieving $c$ 's interface from $\mathcal{R}$.

It is possible to extend the channel's interface of a component without changing its semantics. In Lemma 3.1 (BRJE equivalence notion) the interface $i$ stands for the channel $c$ data type. It states that we can always replace $i$ by one of its conservative extensions (set containment relation), say $i^{\prime}$ (or vice-versa).

Lemma 3.1 (Interface extension). Consider two $\mathcal{B R J C}$ components $T:\langle\mathcal{B}, \mathcal{R} \cup\{($ ch, $i)\}, \mathcal{J} \cup\{i\}, \mathcal{C}\rangle$ and $T^{\prime}:\left\langle\mathcal{B}, \mathcal{R} \cup\left\{\left(\right.\right.\right.$ ch,$\left.\left.\left.i^{\prime}\right)\right\}, \mathcal{J} \cup\left\{i^{\prime}\right\}, \mathcal{C}\right\rangle$, such that $\left\{i, i^{\prime}\right\} \cap \mathcal{J}=\emptyset$. We say that $T \equiv_{\mathcal{B}} T^{\prime}\left(\mathcal{S} \llbracket T \rrbracket=\mathcal{S} \llbracket T^{\prime} \rrbracket\right)$ if and only if $i \subseteq i^{\prime}$ or $i^{\prime} \subseteq i$.

Proof.

$$
\begin{aligned}
& {\left[\mathcal{S} \llbracket T \rrbracket=\mathcal{S} \llbracket T^{\prime} \rrbracket\right]} \\
& \mathcal{S} \llbracket T \rrbracket=(\text { failures }(\mathcal{B}),\{(c, \text { failures }(\mathcal{B} \nmid c)) \mid c \in \mathcal{C}\} \cup\{(\text { ch,failures }(\mathcal{B} \uparrow\{\text { ch.v } \mid v \in i\}))\}) \wedge \\
& \mathcal{S} \llbracket T^{\prime} \rrbracket=\left(\text { failures }(\mathcal{B}),\{(\text { c, failures }(\mathcal{B} \nmid c)) \mid c \in \mathcal{C}\} \cup\left\{\left(\text { ch,failures }\left(\mathcal{B} \uparrow\left\{\text { ch.v } \mid v \in i^{\prime}\right\}\right)\right)\right\}\right) \wedge \\
& \{(\text { ch,failures }(\mathcal{B} \nmid\{\text { ch.v } \mid v \in i\}))\}=\left\{\left(\text { ch,failures }\left(\mathcal{B} \uparrow\left\{\text { ch.v } \mid v \in i^{\prime}\right\}\right)\right)\right\} \\
& \equiv[\text { set theory }] \\
& \text { failures } \left.(\mathcal{B} \uparrow\{\text { ch.v|v } \in i\}))=\operatorname{failures}\left(\mathcal{B} \uparrow\left\{\text { ch.v } \mid v \in i^{\prime}\right\}\right)\right)
\end{aligned}
$$

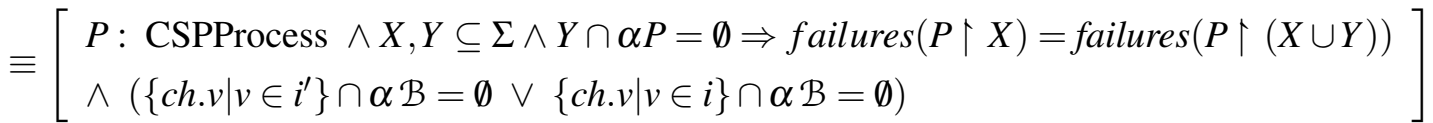

$$
\begin{aligned}
& \{\text { ch. } v \mid v \in i\} \subseteq\left\{c h . v \mid v \in i^{\prime}\right\} \vee\left\{c h . v \mid v \in i^{\prime}\right\} \subseteq\{c h . v \mid v \in i\} \\
& \equiv[\text { set theory }] \\
& i \subseteq i^{\prime} \vee i^{\prime} \subseteq i
\end{aligned}
$$

\section{BRIC refinement}

The notion of $\mathcal{B R J \mathcal { C }}$ refinement plays a central role in our theory. It guarantees many properties required in component compositions, fulfilling the substitutability principle. All the composition operators are monotonic with respect to refinement, as formally established in the end of this section. 
Definition 3.1 ( $\mathcal{B R J C}$ refinement based on failures). Consider two components $T$ and $T^{\prime}$. If we consider $\mathcal{S} \llbracket T \rrbracket=(f, f p)$ and $\mathcal{S} \llbracket T^{\prime} \rrbracket=\left(f^{\prime}, f p^{\prime}\right)$, then we say that $T^{\prime}$ refines $T\left(T \sqsubseteq_{\mathcal{B}} T^{\prime}\right)$, if and only if:

$$
\left(f^{\prime} \subseteq f\right) \wedge\left(\operatorname{dom} f p=\operatorname{dom} f p^{\prime}\right) \wedge\left(\forall c \in \operatorname{dom} f p \bullet f p^{\prime}(c) \subseteq f p(c)\right)
$$

This definition ensures that $T$ and $T^{\prime}$ have the same interaction points. Moreover, it guarantees that the component behaviour of $T^{\prime}$ refines that of $T$, which is equivalent to:

$$
\left(\mathcal{B}_{T} \sqsubseteq_{\mathrm{F}} \mathcal{B}_{T^{\prime}}\right) \wedge\left(\mathcal{C}_{T}=\mathcal{C}_{T^{\prime}}\right) \wedge\left(\forall c: \mathcal{C}_{T} \bullet \mathcal{R}_{T}(c) \subseteq \mathcal{R}_{T^{\prime}}(c)\right)
$$

As addressed in the next section, the $\mathcal{B R J C}$ composition operators are all monotonic with respect to this refinement relation. Therefore, the substitutability principle is fulfilled by the composition rules. To prove this result, we use some straightforward lemmas about the $\mathcal{B R J C}$ refinement relation. The following lemmas show that this relation is transitive and reflexive.

Lemma 3.2 ( $\mathcal{B R J \mathcal { C }}$ refinement is transitive). Consider three $\mathcal{B R J C}$ components $T:\langle\mathcal{B}, \mathcal{R}, \mathcal{J}, \mathcal{C}\rangle$, $T^{\prime}:\left\langle\mathcal{B}^{\prime}, \mathcal{R}^{\prime}, \mathcal{J}^{\prime}, \mathcal{C}^{\prime}\right\rangle$ and $T^{\prime \prime}:\left\langle\mathcal{B}^{\prime \prime}, \mathcal{R}^{\prime \prime}, \mathcal{J}^{\prime \prime}, \mathcal{C}^{\prime \prime}\right\rangle$. If $T \sqsubseteq_{\mathcal{B}} T^{\prime}$ and $T^{\prime} \sqsubseteq_{\mathcal{B}} T^{\prime \prime}$ then $T \sqsubseteq_{\mathcal{B}} T^{\prime \prime}$.

Proof.

$$
\begin{aligned}
& {\left[T \sqsubseteq_{\mathcal{B}} T^{\prime} \wedge T^{\prime} \sqsubseteq_{\mathcal{B}} T^{\prime \prime}\right]} \\
& \text { failures }\left(\mathcal{B}^{\prime}\right) \subseteq \text { failures }(\mathcal{B}) \wedge \mathcal{C}=\mathcal{C}^{\prime} \wedge \forall c \in \mathcal{C} \bullet \mathcal{R}(c) \subseteq \mathcal{R}^{\prime}(c) \wedge \\
& \text { failures }\left(\mathcal{B}^{\prime \prime}\right) \subseteq \text { failures }\left(\mathcal{B}^{\prime}\right) \wedge \mathcal{C}^{\prime}=\mathcal{C}^{\prime \prime} \wedge \forall c \in \mathcal{C}^{\prime} \bullet \mathcal{R}^{\prime}(c) \subseteq \mathcal{R}^{\prime \prime}(c) \\
& \Rightarrow[\text { set theory: transitivity }] \\
& \text { failures }\left(\mathcal{B}^{\prime \prime}\right) \subseteq \text { failures }(\mathcal{B}) \wedge \mathcal{C}=\mathcal{C}^{\prime \prime} \wedge \forall c \in \mathcal{C} \bullet \mathcal{R}(c) \subseteq \mathcal{R}^{\prime \prime}(c) \\
& \Rightarrow[\text { Definition 3.1] } \\
& T \sqsubseteq_{\mathcal{B}} T^{\prime \prime}
\end{aligned}
$$

Lemma 3.3 ( $\mathcal{B} \mathcal{R J C}$ refinement is reflexive). For any $\mathcal{B} \mathcal{R J C}$ component $T:\langle\mathcal{B}, \mathcal{R}, \mathcal{J}, \mathcal{C}\rangle, T \sqsubseteq_{\mathcal{B}} T$. Proof.

[BRJU semantics]

$\mathcal{S} \llbracket T \rrbracket=($ failures $(\mathcal{B}),\{(c$, failures $(\mathcal{B} \nmid c)) \mid c \in \mathcal{C}\})$

$\Rightarrow[$ set theory and failures refinement $\wedge$ Definition 3.1]

$T \sqsubseteq_{\mathcal{B}} T$ 
If a $\mathcal{B R J C}$ component refines a deadlock-free component then it is deadlock-free too. Lemma 3.4 formalises this fact.

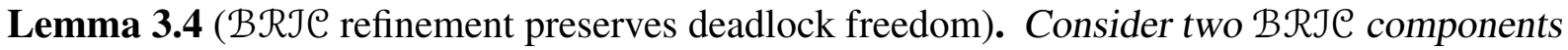
$T:\langle\mathcal{B}, \mathcal{R}, \mathcal{J}, \mathcal{C}\rangle$ and $T^{\prime}:\left\langle\mathcal{B}^{\prime}, \mathcal{R}^{\prime}, \mathcal{J}^{\prime}, \mathcal{C}^{\prime}\right\rangle$. If $T \sqsubseteq_{\mathcal{B}} T^{\prime}$ and $T$ is a deadlock-free contract, so is $T^{\prime}$.

Proof.

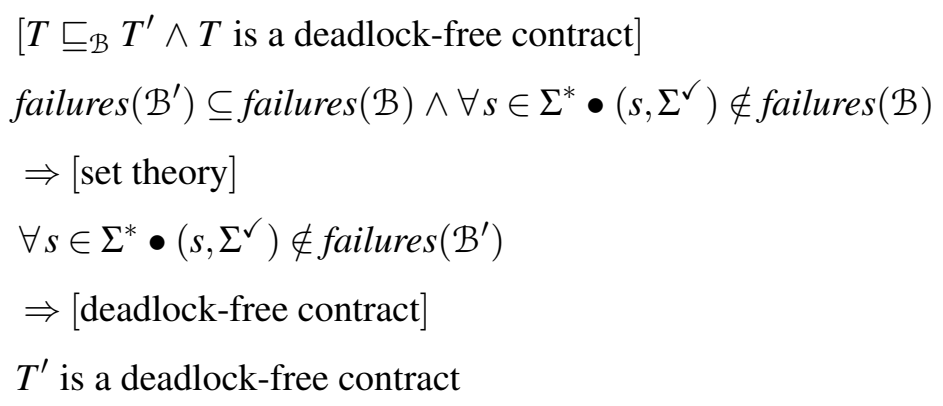

The behaviour of a $\mathcal{B R J C}$ component is given by an I/O process and, since our relation relies on the CSP failures model, it is important to stablish what behavioural properties are preserved by refinement in this model. If an I/O process refines an I/O confluent process then it is $\mathrm{I} / \mathrm{O}$ confluent too. Theorem 3.1 formalises this fact.

Theorem 3.1 (Failures refinement preserves I/O confluence). Consider two I/O processes $T$ and $T^{\prime}$. If $T$ is $I / O$ confluent and $T \sqsubseteq_{\mathrm{F}} T^{\prime}$ then so is $T^{\prime}$.

Proof.

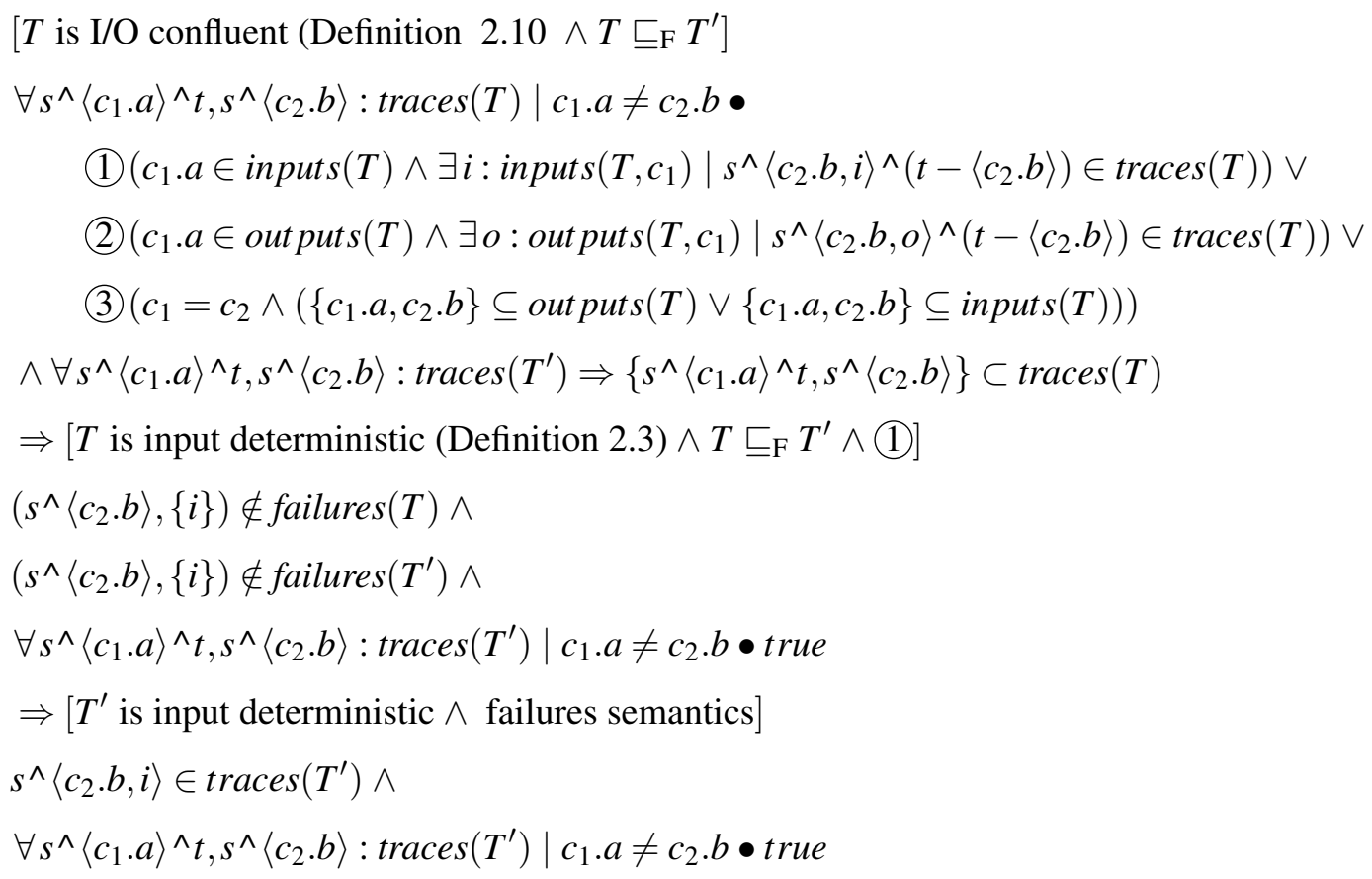




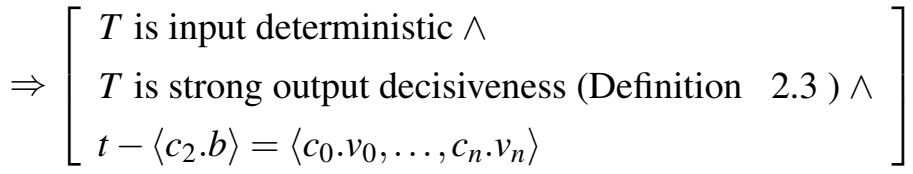

$$
\begin{aligned}
& \left(s^{\wedge}\left\langle c_{2} . b, i\right\rangle,\left\{c_{0} \cdot v_{0}\right\}\right) \notin \text { failures }(T) \vee \\
& \left(\left(s^{\wedge}\left\langle c_{2} . b, i\right\rangle, \text { outputs }\left(c_{0}, T\right)\right) \notin \text { failures }(T) \wedge\right. \\
& \left.\left(s^{\wedge}\left\langle c_{2} \cdot b, i\right\rangle, \text { out puts }\left(c_{0}, T\right) \backslash\left\{c_{0} \cdot v_{0}\right\}\right) \in \text { failures }(T)\right) \wedge \\
& \forall s^{\wedge}\left\langle c_{1} . a\right\rangle^{\wedge} t, s^{\wedge}\left\langle c_{2} . b\right\rangle: \operatorname{traces}\left(T^{\prime}\right) \mid c_{1} . a \neq c_{2} . b \bullet \text { true } \\
& \Rightarrow \text { [by induction on elements of } t-\left\langle c_{2} . b\right\rangle \wedge T \sqsubseteq_{\mathrm{F}} T^{\prime} \text { ] } \\
& s^{\wedge}\left\langle c_{2} \cdot b, i, c_{0} \cdot v_{0}\right\rangle \in \operatorname{traces}\left(T^{\prime}\right) \wedge \\
& s^{\wedge}\left\langle c_{2} \cdot b, i\right\rangle^{\wedge}\left(t-\left\langle c_{2} \cdot b\right\rangle\right) \in \operatorname{traces}\left(T^{\prime}\right) \\
& \Rightarrow \forall s^{\wedge}\left\langle c_{1} . a\right\rangle^{\wedge} t, s^{\wedge}\left\langle c_{2} . b\right\rangle: \operatorname{traces}\left(T^{\prime}\right) \mid c_{1} . a \neq c_{2} . b \bullet \\
& \left(c_{1} . a \in \operatorname{inputs}\left(T^{\prime}\right) \wedge \exists i: \operatorname{inputs}\left(T^{\prime}, c_{1}\right) \mid s^{\wedge}\left\langle c_{2} . b, i\right\rangle^{\wedge}\left(t-\left\langle c_{2} . b\right\rangle\right) \in \operatorname{traces}\left(T^{\prime}\right)\right) \vee \text { true } \\
& \Rightarrow \text { [ applying the same previous reasoning for outputs } \wedge \text { (2)] } \\
& s^{\wedge}\left\langle c_{2} . b, o\right\rangle^{\wedge}\left(t-\left\langle c_{2} . b\right\rangle\right) \in \operatorname{traces}\left(T^{\prime}\right) \\
& \Rightarrow \forall s^{\wedge}\left\langle c_{1} . a\right\rangle^{\wedge} t, s^{\wedge}\left\langle c_{2} . b\right\rangle: \operatorname{traces}\left(T^{\prime}\right) \mid c_{1} . a \neq c_{2} . b \bullet \\
& \left(c_{1} . a \in \operatorname{inputs}\left(T^{\prime}\right) \wedge \exists i: \operatorname{inputs}\left(T^{\prime}, c_{1}\right) \mid s^{\wedge}\left\langle c_{2} . b, i\right\rangle^{\wedge}\left(t-\left\langle c_{2} \cdot b\right\rangle\right) \in \operatorname{traces}\left(T^{\prime}\right)\right) \vee \\
& \left(c_{1} . a \in \text { outputs }\left(T^{\prime}\right) \wedge \exists o: \text { outputs }\left(T^{\prime}, c_{1}\right) \mid s^{\wedge}\left\langle c_{2} . b, o\right\rangle^{\wedge}\left(t-\left\langle c_{2} . b\right\rangle\right) \in \operatorname{traces}\left(T^{\prime}\right)\right) \vee \text { true } \\
& \Rightarrow \text { [ choices between inputs and outputs are on different channels RAMOS (2011) } \wedge \text { (3)] } \\
& \left\{c_{1} \cdot a, c_{2} \cdot b\right\} \subseteq \operatorname{inputs}\left(T^{\prime}\right) \vee\left\{c_{1} \cdot a, c_{2} \cdot b\right\} \subseteq \text { outputs }\left(T^{\prime}\right) \\
& \Rightarrow \forall s^{\wedge}\left\langle c_{1} . a\right\rangle^{\wedge} t, s^{\wedge}\left\langle c_{2} . b\right\rangle: \operatorname{traces}\left(T^{\prime}\right) \mid c_{1} . a \neq c_{2} . b \bullet \\
& \left(c_{1} . a \in \operatorname{inputs}\left(T^{\prime}\right) \wedge \exists i: \operatorname{inputs}\left(T^{\prime}, c_{1}\right) \mid s^{\wedge}\left\langle c_{2} . b, i\right\rangle^{\wedge}\left(t-\left\langle c_{2} . b\right\rangle\right) \in \operatorname{traces}\left(T^{\prime}\right)\right) \vee \\
& \left(c_{1} . a \in \text { outputs }\left(T^{\prime}\right) \wedge \exists o: \text { outputs }\left(T^{\prime}, c_{1}\right) \mid s^{\wedge}\left\langle c_{2} \cdot b, o\right\rangle^{\wedge}\left(t-\left\langle c_{2} . b\right\rangle\right) \in \operatorname{traces}\left(T^{\prime}\right)\right) \vee \\
& \left(c_{1}=c_{2} \wedge\left(\left\{c_{1} \cdot a, c_{2} \cdot b\right\} \subseteq \text { outputs }\left(T^{\prime}\right) \vee\left\{c_{1} \cdot a, c_{2} \cdot b\right\} \subseteq \text { inputs }\left(T^{\prime}\right)\right)\right) \\
& \Rightarrow[\mathrm{I} / \mathrm{O} \text { confluence (Definition 2.10)] } \\
& T^{\prime} \text { is I/O confluent }
\end{aligned}
$$

A communication protocol is a deadlock-free I/O process that is generally associated to the behaviour exhibited by some $\mathcal{B R J C}$ components when restricted to communicate through a particular channel. Two communication protocols are conjugate if they can consume the outputs of each other when put in parallel. This is a relevant property to the composition rules (except interleaving) and it is preserved by failures refinement. Lemma 3.5 formalises this fact.

Lemma 3.5 (Failures refinement and protocol conjugation). Consider $T, R$ and $T^{\prime}$ three communication protocols. If $T$ and $R$ are conjugate and $T \sqsubseteq_{\mathrm{F}} T^{\prime}$ then $T^{\prime}$ and $R$ are also conjugate. 
Proof.

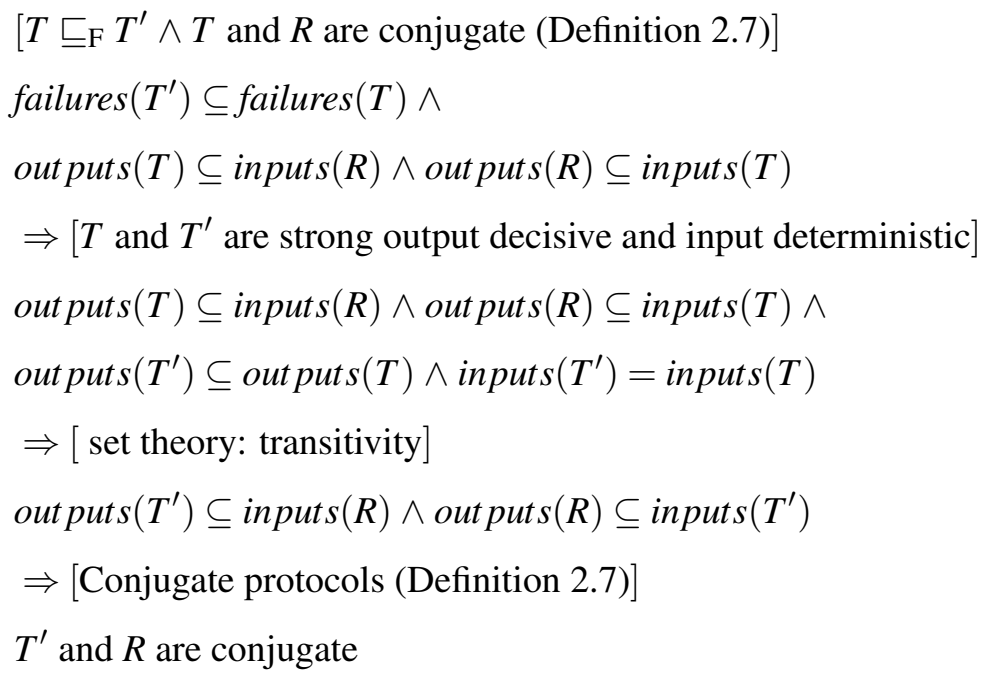

Two communication protocols are strong compatible if, when put in parallel, the outputs of each process are accepted by the other in all scenarios. This is a relevant property to the composition rules (except interleaving) and it is preserved by failures refinement. This is ensured by the following lemma.

Lemma 3.6 (Failures refinement and strong protocol compatibility). Consider $T, R$ and $T^{\prime}$ three communication protocols. If $T \approx R$ (strong protocol compatible) and $T \sqsubseteq_{\mathrm{F}} T^{\prime}$ then $T^{\prime} \approx R$.

Proof.

$$
\begin{aligned}
& {\left[T \approx R(\text { Definition } 2.9) \wedge T \sqsubseteq_{\mathrm{F}} T^{\prime}\right]} \\
& \forall s \in \operatorname{traces}\left(T^{\prime}\right) \cap \operatorname{traces}(R) \bullet s \in \operatorname{traces}(T) \wedge \\
& \forall s: \operatorname{traces}(T) \cap \operatorname{traces}(R) \bullet(\text { outputs }(T / s) \cup \text { outputs }(R / s) \neq \emptyset) \wedge \\
& \quad(\text { outputs }(T / s) \subseteq \operatorname{inputs}(R / s) \wedge \text { outputs }(R / s) \subseteq \operatorname{inputs}(T / s)) \\
& \left.\Rightarrow \quad\left[T \text { and } T^{\prime} \text { are input deterministic (Definition } 2.4\right)\right] \\
& \forall s \in \operatorname{traces}\left(T^{\prime}\right) \cap \operatorname{traces}(R) \bullet s \in \operatorname{traces}(T) \wedge \\
& \forall s: \operatorname{traces}(T) \cap \text { traces }(R) \bullet(\text { outputs }(T / s) \cup \text { outputs }(R / s) \neq \emptyset) \wedge \\
& \quad(\text { outputs }(T / s) \subseteq \text { inputs }(R / s) \wedge \text { outputs }(R / s) \subseteq \text { inputs }(T / s)) \\
& \wedge \text { inputs }(T / s)=\text { inputs }\left(T^{\prime} / s\right) \\
& \Rightarrow \quad\left[T \text { and } T^{\prime} \text { are strong output decisiveness }(\text { Definition } 2.4)\right] \\
& \forall s \in \operatorname{traces}\left(T^{\prime}\right) \cap \operatorname{traces}(R) \bullet s \in \operatorname{traces}(T) \wedge \\
& \forall s: \operatorname{traces}(T) \cap \text { traces }(R) \bullet(\text { outputs }(T / s) \cup \text { outputs }(R / s) \neq \emptyset) \wedge \\
& \quad(\text { outputs }(T / s) \subseteq \text { inputs }(R / s) \wedge \text { outputs }(R / s) \subseteq \text { inputs }(T / s))
\end{aligned}
$$




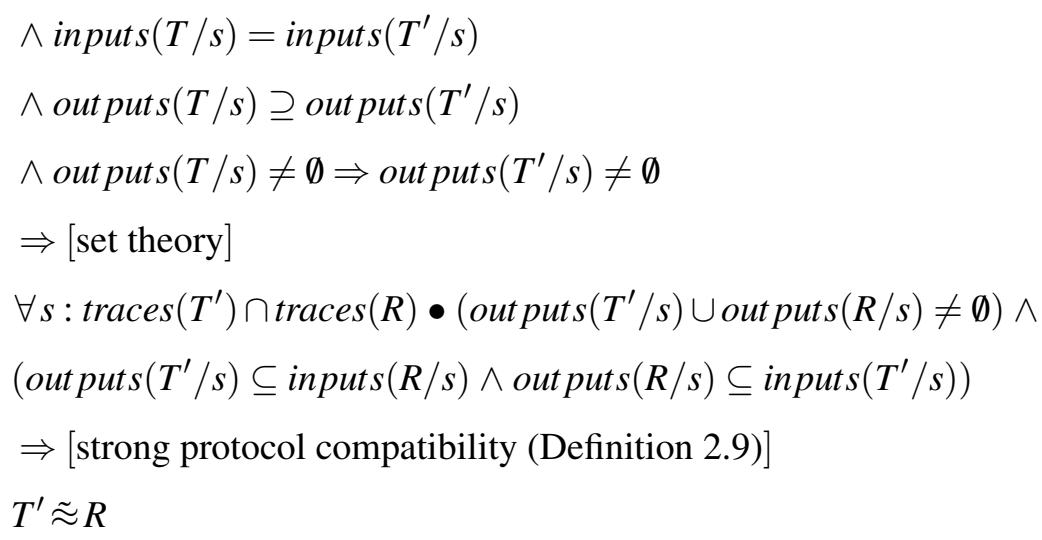

An I/O process has the Finite Output Property (FOP) when it cannot perform an infinite sequence of outputs without an input. This is a relevant property to the composition rules and it is preserved by failures refinement, as formalised by the following lemma.

Lemma 3.7 (Failures refinement preserves Finite Output Property). Consider $T$ and $T^{\prime}$ two $I / O$ processes and $\mathcal{C}$ and $\mathcal{C}^{\prime}$ the set of channels used in $T$ and $T^{\prime}$, respectively. If $T$ satisfies the Finite Output Property, $T^{\prime}$ is divergence free and $T \sqsubseteq_{\mathrm{F}} T^{\prime}$ then $T^{\prime}$ also satisfies the Finite Output Property.

Proof.

$\left[T\right.$ satisfies FOP (Finite Output Property, Definition 2.11) $\left.\wedge T \sqsubseteq_{\mathrm{F}} T^{\prime}\right]$

$\mathrm{C}^{\prime} \subseteq \mathcal{C} \wedge \forall c: \mathcal{C} \bullet T \backslash$ outputs $(T, c)$ is divergence free

$\Rightarrow$ [hide semantics (ROSCOE, 1998)]

$\mathcal{C}^{\prime} \subseteq \mathcal{C} \wedge \forall c: \mathcal{C} \bullet T \backslash$ outputs $(T, c)$ is divergence free $\wedge$

$\forall c: \mathcal{C}^{\prime} \bullet T \backslash$ outputs $(T, c) \sqsubseteq_{\mathrm{F}} T^{\prime} \backslash$ outputs $(T, c)$

$\Rightarrow\left[T\right.$ and $T^{\prime}$ satisfy strong output decisiveness (Definition 2.4)]

$\mathcal{C}^{\prime} \subseteq \mathcal{C} \wedge \forall c: \mathcal{C} \bullet T \backslash$ outputs $(T, c)$ is divergence free $\wedge$

$\forall c: \mathrm{C}^{\prime} \bullet T \backslash$ outputs $(T, c) \sqsubseteq_{\mathrm{F}} T^{\prime} \backslash$ outputs $(T, c) \wedge$

$\forall c: \mathcal{C}^{\prime} \bullet$ outputs $\left(T^{\prime}, c\right) \subseteq$ outputs $(T, c)$

$\Rightarrow\left[D(c)=\right.$ outputs $(T, c) \backslash$ outputs $\left.\left(T^{\prime}, c\right) \mid \operatorname{dom} D=\mathrm{C}\right]$

$\mathrm{C}^{\prime} \subseteq \mathcal{C} \wedge \forall c: \mathcal{C} \bullet T \backslash$ outputs $(T, c)$ is divergence free $\wedge$

$\forall c: \mathcal{C}^{\prime} \bullet T \backslash$ outputs $(T, c) \sqsubseteq_{\mathrm{F}} T^{\prime} \backslash$ outputs $\left(T^{\prime}, c\right) \cup D(c) \wedge$

$\forall c: \mathcal{C}^{\prime} \bullet$ outputs $\left(T^{\prime}, c\right) \subseteq$ outputs $(T, c)$

$\Rightarrow\left[P \backslash X \equiv_{\mathrm{F}} P \mid \alpha P \cap X=\emptyset \wedge P:\right.$ CSPProcess $\left.\wedge X \subseteq \Sigma\right]$

$\mathrm{C}^{\prime} \subseteq \mathcal{C} \wedge \forall c: \mathcal{C} \bullet T \backslash$ outputs $(T, c)$ is divergence free $\wedge$

$\forall c: \mathcal{C}^{\prime} \bullet T \backslash$ outputs $(T, c) \sqsubseteq_{\mathrm{F}} T^{\prime} \backslash$ outputs $\left(T^{\prime}, c\right)$ 
$\Rightarrow\left[T^{\prime}\right.$ is divergence free $\wedge$ divergence and refinement (ROSCOE, 1998)]

$\forall c: \mathrm{C}^{\prime} \bullet T^{\prime} \backslash$ outputs $\left(T^{\prime}, c\right)$ is divergence free

$\Rightarrow T^{\prime}$ satisfies the FOP

A buffering self injection I/O process can stablish a communication between two of its channels via an one-place-buffer without deadlock. This is a necessary condition for the reflexive composition rule. Theorem 3.2 formalises the fact that failures refinement preserves this property.

Theorem 3.2 (Failures refinement and self-injection compatibility). Consider two deadlock-free I/O processes $T$ and $T^{\prime}$. If $T \uparrow\{|c, z|\}$ is buffered self-injection compatible and $T \sqsubseteq_{\mathrm{F}} T^{\prime}$ then $T^{\prime} \uparrow\{|c, z|\}$ is also buffered self-injection compatible ( $c$ and $z$ are I/O channels).

Proof.

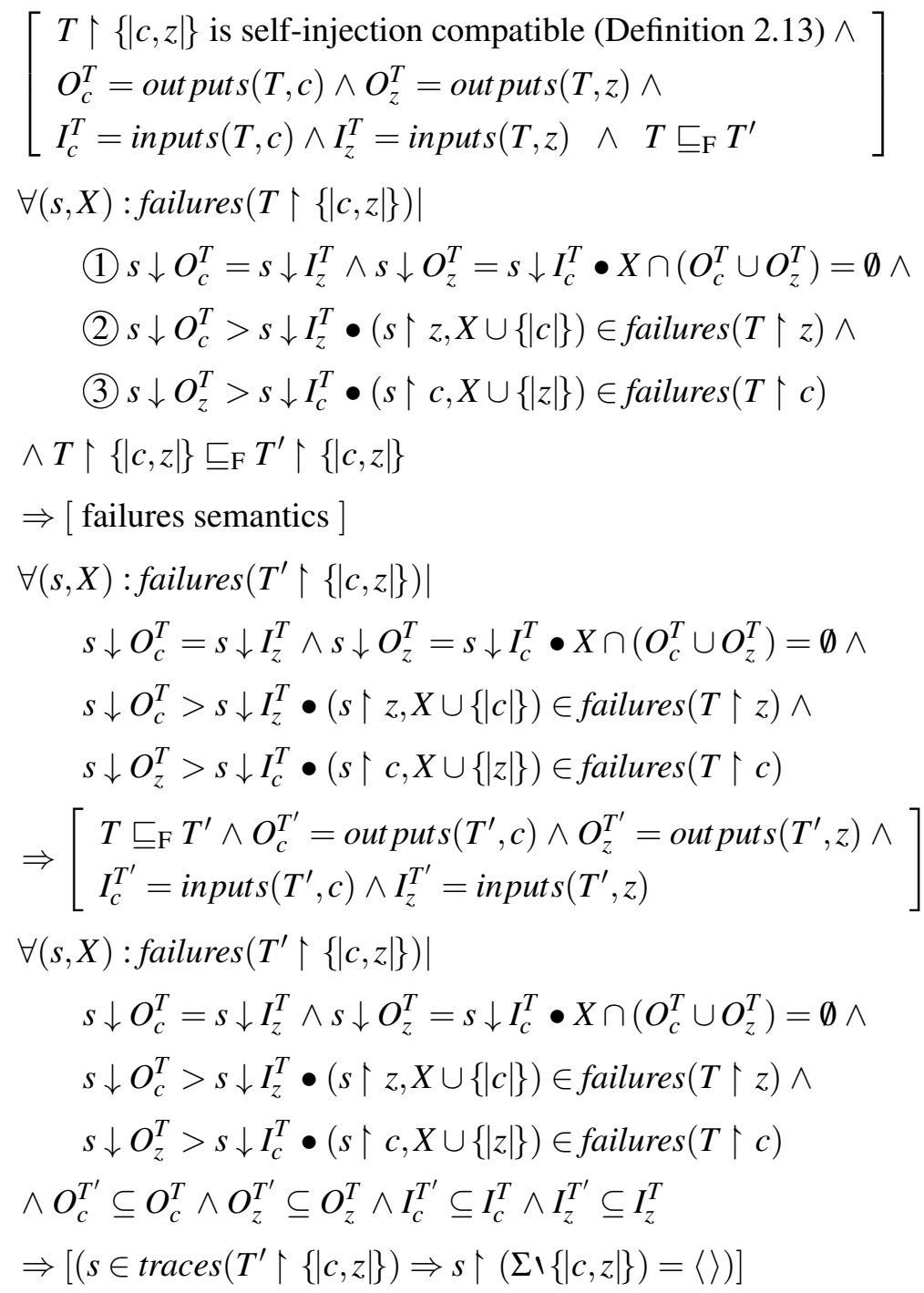




$$
\begin{aligned}
& \forall(s, X): \text { failures }\left(T^{\prime} \uparrow\{|c, z|\}\right) \mid \\
& s \downarrow O_{c}^{T}=s \downarrow I_{z}^{T} \wedge s \downarrow O_{z}^{T}=s \downarrow I_{c}^{T} \bullet X \cap\left(O_{c}^{T} \cup O_{z}^{T}\right)=\emptyset \wedge \\
& s \downarrow O_{c}^{T}>s \downarrow I_{z}^{T} \bullet(s \uparrow z, X \cup\{|c|\}) \in \text { failures }(T \nmid z) \wedge \\
& s \downarrow O_{z}^{T}>s \downarrow I_{c}^{T} \bullet(s \uparrow c, X \cup\{|z|\}) \in \text { failures }(T \uparrow c) \\
& \wedge O_{c}^{T^{\prime}} \subseteq O_{c}^{T} \wedge O_{z}^{T^{\prime}} \subseteq O_{z}^{T} \wedge I_{c}^{T^{\prime}} \subseteq I_{c}^{T} \wedge I_{z}^{T^{\prime}} \subseteq I_{z}^{T} \wedge \\
& \left(s \downarrow O_{c}^{T}=s \downarrow I_{z}^{T} \wedge s \downarrow O_{z}^{T}=s \downarrow I_{c}^{T}\right) \Rightarrow\left(s \downarrow O_{c}^{T^{\prime}}=s \downarrow I_{z}^{T^{\prime}} \wedge s \downarrow O_{z}^{T^{\prime}}=s \downarrow I_{c}^{T^{\prime}}\right) \wedge \\
& \left(X \cap\left(O_{c}^{T} \cup O_{z}^{T}\right)=\emptyset\right) \Rightarrow\left(X \cap\left(O_{c}^{T^{\prime}} \cup O_{z}^{T^{\prime}}\right)=\emptyset\right) \\
& \Rightarrow[1 \wedge \wedge \text { set theory] } \\
& \forall(s, X): \text { failures }\left(T^{\prime} \uparrow\{|c, z|\}\right) \mid \\
& s \downarrow O_{c}^{T^{\prime}}=s \downarrow I_{z}^{T^{\prime}} \wedge s \downarrow O_{z}^{T^{\prime}}=s \downarrow I_{c}^{T^{\prime}} \bullet X \cap\left(O_{c}^{T^{\prime}} \cup O_{z}^{T^{\prime}}\right)=\emptyset \wedge \\
& s \downarrow O_{c}^{T}>s \downarrow I_{z}^{T} \bullet(s \uparrow z, X \cup\{|c|\}) \in \text { failures }(T \nmid z) \wedge \\
& s \downarrow O_{z}^{T}>s \downarrow I_{c}^{T} \bullet(s \uparrow c, X \cup\{|z|\}) \in \text { failures }(T \uparrow c) \\
& \wedge O_{c}^{T^{\prime}} \subseteq O_{c}^{T} \wedge O_{z}^{T^{\prime}} \subseteq O_{z}^{T} \wedge I_{c}^{T^{\prime}} \subseteq I_{c}^{T} \wedge I_{z}^{T^{\prime}} \subseteq I_{z}^{T} \\
& \Rightarrow\left[(2) \wedge\left(s \downarrow O_{c}^{T}>s \downarrow I_{z}^{T} \Rightarrow s \downarrow O_{c}^{T^{\prime}}>s \downarrow I_{z}^{T^{\prime}}\right)\right] \\
& \forall(s, X): \text { failures }\left(T^{\prime} \uparrow\{|c, z|\}\right) \mid \\
& s \downarrow O_{c}^{T^{\prime}}=s \downarrow I_{z}^{T^{\prime}} \wedge s \downarrow O_{z}^{T^{\prime}}=s \downarrow I_{c}^{T^{\prime}} \bullet X \cap\left(O_{c}^{T^{\prime}} \cup O_{z}^{T^{\prime}}\right)=\emptyset \wedge \\
& s \downarrow O_{c}^{T^{\prime}}>s \downarrow I_{z}^{T^{\prime}} \bullet(s \uparrow z, X \cup\{|c|\}) \in \operatorname{failures}\left(T^{\prime} \uparrow z\right) \wedge \\
& s \downarrow O_{z}^{T}>s \downarrow I_{c}^{T} \bullet(s \uparrow c, X \cup\{|z|\}) \in \text { failures }(T \uparrow c) \\
& \wedge O_{c}^{T^{\prime}} \subseteq O_{c}^{T} \wedge O_{z}^{T^{\prime}} \subseteq O_{z}^{T} \wedge I_{c}^{T^{\prime}} \subseteq I_{c}^{T} \wedge I_{z}^{T^{\prime}} \subseteq I_{z}^{T} \\
& \Rightarrow\left[\begin{array}{l}
\left(3 \wedge\left(T \sqsubseteq_{\mathrm{F}} T^{\prime}\right) \Rightarrow\left(T \uparrow z \sqsubseteq_{\mathrm{F}} T^{\prime} \uparrow z\right) \wedge\right. \\
(s, X) \in \text { failures }\left(T^{\prime} \uparrow z\right) \Rightarrow(s, X \cup Y) \in \text { failures }\left(T^{\prime} \uparrow z\right) \mid Y \cap\{|z|\}=\emptyset
\end{array}\right] \\
& \forall(s, X): \text { failures }\left(T^{\prime} \uparrow\{|c, z|\}\right) \mid \\
& s \downarrow O_{c}^{T^{\prime}}=s \downarrow I_{z}^{T^{\prime}} \wedge s \downarrow O_{z}^{T^{\prime}}=s \downarrow I_{c}^{T^{\prime}} \bullet X \cap\left(O_{c}^{T^{\prime}} \cup O_{z}^{T^{\prime}}\right)=\emptyset \wedge \\
& s \downarrow O_{c}^{T^{\prime}}>s \downarrow I_{z}^{T^{\prime}} \bullet(s \uparrow z, X \cup\{|c|\}) \in \operatorname{failures}\left(T^{\prime} \uparrow z\right) \wedge \\
& s \downarrow O_{z}^{T}>s \downarrow I_{c}^{T} \bullet(s \uparrow c, X \cup\{|z|\}) \in \text { failures }(T \uparrow c) \\
& \wedge O_{c}^{T^{\prime}} \subseteq O_{c}^{T} \wedge O_{z}^{T^{\prime}} \subseteq O_{z}^{T} \wedge I_{c}^{T^{\prime}} \subseteq I_{c}^{T} \wedge I_{z}^{T^{\prime}} \subseteq I_{z}^{T} \\
& \wedge(s \uparrow z, X \cup\{|c|\}) \in \text { failures }\left(T^{\prime} \uparrow z\right) \\
& \Rightarrow \text { [applying the same reasoning for } s \downarrow O_{z}^{T}>s \downarrow I_{c}^{T} \text { ] } \\
& \forall(s, X): \text { failures }\left(T^{\prime} \uparrow\{|c, z|\}\right) \mid \\
& s \downarrow O_{c}^{T^{\prime}}=s \downarrow I_{z}^{T^{\prime}} \wedge s \downarrow O_{z}^{T^{\prime}}=s \downarrow I_{c}^{T^{\prime}} \bullet X \cap\left(O_{c}^{T^{\prime}} \cup O_{z}^{T^{\prime}}\right)=\emptyset \wedge \\
& s \downarrow O_{c}^{T^{\prime}}>s \downarrow I_{z}^{T^{\prime}} \bullet(s \uparrow z, X \cup\{|c|\}) \in \operatorname{failures}\left(T^{\prime} \uparrow z\right) \wedge \\
& s \downarrow O_{z}^{T^{\prime}}>s \downarrow I_{c}^{T^{\prime}} \bullet(s \uparrow c, X \cup\{|z|\}) \in \text { failures }\left(T^{\prime} \uparrow c\right) \\
& \Rightarrow \text { [buffer self-injection compatibility (Definition 2.13)] } \\
& T^{\prime} \uparrow\{|c, z|\} \text { is self-injection compatible }
\end{aligned}
$$


So far we have discussed the properties and relations between $\mathrm{I} / \mathrm{O}$ processes that are preserved by failures refinement. Since component behaviour is specified in terms of I/O processes and component refinement in terms of failures, these previous results play an important role in proving the monotonicity of $\mathcal{B R J C}$ refinement. A final result, at this time at the component level, is also needed to prove the monotonicity of $\sqsubseteq_{\mathcal{B}}$.

Two channels of a $\mathcal{B R J C}$ component are decoupled if the communication through them behaves as communication between channels of distinct processes. This is a necessary condition for the feedback composition rule. Lemma 3.8 formalises the fact that our refinement relation for $\mathcal{B} \mathcal{R J C}$ preserves this property.

Lemma 3.8 (BRJC refinement and decoupled channels). Consider $T:\langle\mathcal{B}, \mathcal{R}, \mathcal{J}, \mathcal{C}\rangle$ and $T^{\prime}$ : $\left\langle\mathcal{B}^{\prime}, \mathcal{R}^{\prime}, \mathcal{J}^{\prime}, \mathcal{C}^{\prime}\right\rangle$ two $\mathcal{B} \mathcal{R J C}$ components. If $T \sqsubseteq_{\mathcal{B}} T^{\prime}$ and $\left\{c_{1}, c_{2}\right\} \subseteq \mathcal{C}$ are decoupled in $T$ then $\left\{c_{1}, c_{2}\right\} \subseteq \mathcal{C}^{\prime}$ are decoupled in $T^{\prime}$.

Proof.

$$
\begin{aligned}
& {\left[T \sqsubseteq_{\mathcal{B}} T^{\prime} \wedge\left\{c_{1}, c_{2}\right\} \subseteq \mathcal{C} \text { are decoupled in } T \text { (Definition 2.12) }\right]} \\
& \mathcal{C}=\mathcal{C}^{\prime} \wedge\left\{c_{1}, c_{2}\right\} \subseteq \mathcal{C}^{\prime} \wedge \mathcal{B} \sqsubseteq_{\mathrm{F}} \mathcal{B}^{\prime} \wedge \\
& \mathcal{B} \uparrow\left\{c_{1}, c_{2}\right\} \equiv_{\mathrm{F}} \mathcal{B} \uparrow\left\{\left|c_{1}\right|\right\}|| \mathcal{B} \uparrow\left\{\left|c_{2}\right|\right\} \\
& \Rightarrow[\text { failures refinement semantics }] \\
& \mathcal{B}^{\prime} \uparrow\left\{\left|c_{1}, c_{2}\right|\right\} \equiv_{\mathrm{F}} \mathcal{B}^{\prime} \uparrow\left\{\left|c_{1}\right|\right\}|| \mathcal{B}^{\prime} \uparrow\left\{\left|c_{2}\right|\right\} \\
& \Rightarrow[\text { Definition 2.12] } \\
& \left\{c_{1}, c_{2}\right\} \subseteq \mathcal{C}^{\prime} \text { are decoupled in } T^{\prime}
\end{aligned}
$$

In this section we have established how our $\mathcal{B R J C}$ refinement relation, as well as failures refinement, relate with behavioural properties relevant for the composition rules. The next section systematically collects these results to prove that the $\mathcal{B R J C}$ composition operators are all monotonic with respect to the $\mathcal{B} \mathcal{R J C}$ refinement relation.

\section{Monotonicity of BRIC operators with respect to refinement}

The $\mathcal{B R J C}$ composition operators are monotonic with respect to refinement. Although refinement is based on set inclusion (failures and communication channels), properties such as self-injection compatibility (Theorem 3.2) and I/O confluence (Theorem 3.1), alongside the $\mathcal{B R J C}$ composition rules provisos are relevant to stablish monotonicity, which is proved by the following lemmas.

Lemma 3.9 (Interleave composition is monotonic with respect to $\mathcal{B} \mathcal{R J C}$ refinement). Consider $T, R$ and $T^{\prime}$ three component contracts, such that $\mathcal{C}_{T} \cap \mathcal{C}_{R}=\emptyset$. If $T$ and $R$ are deadlock free contracts and $T \sqsubseteq_{\mathcal{B}} T^{\prime}$, then $(T[|| \mid] R) \sqsubseteq_{\mathcal{B}}\left(T^{\prime}[\|||] R\right)$. 


\section{Proof.}

$\left[\mathcal{C}_{T} \cap \mathcal{C}_{R}=\emptyset \wedge T\right.$ and $R$ are deadlock free $\wedge T \sqsubseteq_{\mathcal{B}} T^{\prime} \wedge$ Lemma 3.4 $]$

$T^{\prime}$ is a deadlock free contract $\wedge\left(\mathcal{C}_{T}=\mathcal{C}_{T^{\prime}}\right) \wedge\left(\mathcal{C}_{T^{\prime}} \cap \mathfrak{C}_{R}=\emptyset\right)$

$\Rightarrow$ [interleave composition (Definition 2.16) $\wedge$ deadlock free interleave composition (Theorem 2.1)]

$T^{\prime}$ is a deadlock free contract $\wedge\left(\mathcal{C}_{T}=\mathcal{C}_{T^{\prime}}\right) \wedge$

$(T[\|\|] R)$ and $\left(T^{\prime}[\|\|] R\right)$ are deadlock free contracts

$\Rightarrow\left[\mathcal{B}_{(T[\|] R)}=\left(\mathcal{B}_{T} \mid \| \mathcal{B}_{R}\right) \wedge \mathcal{B}_{\left.\left(T^{\prime}[\|]\right] R\right)}=\left(\mathcal{B}_{T^{\prime}} \| \mid \mathcal{B}_{R}\right) \wedge\right.$ monotonicity of $\left.\sqsubseteq_{\mathrm{F}}\right]$

$\mathcal{B}_{(T[\|] \mid R)} \sqsubseteq_{\mathrm{F}} \mathcal{B}_{\left.\left(T^{\prime}[\|]\right] R\right)} \wedge\left(\mathcal{C}_{T}=\mathcal{C}_{T^{\prime}}\right) \wedge\left(\mathcal{C}_{T^{\prime}} \cap \mathcal{C}_{R}=\emptyset\right)$

$\Rightarrow$ [interleave composition (Definition 2.16) $\wedge$ set theory]

$\mathcal{B}_{(T[\|]] R)} \sqsubseteq_{\mathrm{F}} \mathcal{B}_{\left(T^{\prime}[\|\|] R\right)} \wedge \mathcal{C}_{(T[\|\|] R)}=\mathcal{C}_{\left(T^{\prime}[\|\|] R\right)}$

$\Rightarrow[$ refinement $($ Definition 3.1) $\wedge$ interleave composition (Definition 2.16) $]$

$(T[\|||]) \sqsubseteq_{\mathcal{B}}\left(T^{\prime}[\|\mid\|] R\right)$

This lemma requires, among other conditions, $T$ and $R$ to be deadlock-free with disjoint channels $\left(\mathcal{C}_{T} \cap \mathfrak{C}_{R}=\emptyset\right)$. These conditions guarantee that the composition of $T$ and $R$ communicates without interference from each other without introducing deadlock; these conditions comes from Definition 2.16 and are explained in detail by RAMOS (2011).

Lemma 3.10 (Communication composition is monotonic with respect to $\mathcal{B} \mathcal{R J C}$ refinement). Consider $T, R$ and $T^{\prime}$ three component contracts and $N_{I O}$ a bijection, such that $\mathcal{C}_{T} \cap \mathcal{C}_{R}=\emptyset$, $c \in \mathcal{C}_{T}, z \in \mathcal{C}_{R}$ and $T \uparrow c\left[\left[N_{I O}^{c \rightarrow z}\right]\right]$ and $R \uparrow z\left[\left[N_{I O}^{z \rightarrow c}\right]\right]$ are $I / O$ confluent strong compatible and satisfy the finite output property. If $T \sqsubseteq_{\mathcal{B}} T^{\prime}$, then $T([c \leftrightarrow z] R) \sqsubseteq_{\mathcal{B}}\left(T^{\prime}[c \leftrightarrow z] R\right)$.

Proof.

$\left[\mathcal{C}_{T} \cap \mathfrak{C}_{R}=\emptyset \wedge c \in \mathcal{C}_{T} \wedge z \in \mathcal{C}_{R} \wedge(T[c \leftrightarrow z] R)\right.$ is a $\mathcal{B} \mathcal{R J C}$ component $\left.\wedge T \sqsubseteq_{\mathcal{B}} T^{\prime}\right]$

$\left(\mathcal{B}_{T} \uparrow c\right) \sqsubseteq_{\mathrm{F}}\left(\mathcal{B}_{T^{\prime}} \uparrow c\right) \wedge \mathfrak{C}_{T}=\mathfrak{C}_{T^{\prime}} \wedge\left(\mathcal{C}_{T^{\prime}} \cap \mathfrak{C}_{R}\right)=\emptyset$

$\Rightarrow\left[N_{I O}^{c \rightarrow z}\right.$ is a bijection $\wedge$ failures refinement semantics $]$

$\left(\mathcal{B}_{T} \uparrow c\right) \sqsubseteq_{\mathrm{F}}\left(\mathcal{B}_{T^{\prime}} \uparrow c\right) \wedge \mathfrak{C}_{T}=\mathcal{C}_{T^{\prime}} \wedge \mathcal{C}_{T^{\prime}} \cap \mathfrak{C}_{R}=\emptyset \wedge$

$\left(\left(\mathcal{B}_{T} \uparrow c\right)\left[\left[N_{I O}^{c \rightarrow z}\right]\right]\right) \sqsubseteq_{\mathrm{F}}\left(\left(\mathcal{B}_{T^{\prime}} \uparrow c\right)\left[\left[N_{I O}^{c \rightarrow z}\right]\right]\right)$

$\Rightarrow\left[\Xi_{\mathrm{F}}\right.$ preserves I/O confluence (Theorem 3.1)]

$\left(\mathcal{B}_{T} \uparrow c\right) \sqsubseteq_{\mathrm{F}}\left(\mathcal{B}_{T^{\prime}} \uparrow c\right) \wedge \mathcal{C}_{T}=\mathcal{C}_{T^{\prime}} \wedge \mathcal{C}_{T^{\prime}} \cap \mathcal{C}_{R}=\emptyset \wedge$

$\left(\left(\mathcal{B}_{T^{\prime}}^{\uparrow} c\right)\left[\left[N_{I O}^{c \rightarrow z}\right]\right]\right)$ is I/O confluent

$\Rightarrow\left[\complement_{\mathrm{F}}\right.$ preserves strong protocol compatibility (Lemma 3.6) $\left.\wedge\left(\left(\mathcal{B}_{T} \uparrow c\right)\left[\left[N_{I O}^{c \rightarrow z}\right]\right]\right) \approx\left(\left(\mathcal{B}_{R} \uparrow z\right)\left[\left[N_{I O}^{z \rightarrow c}\right]\right]\right)\right]$

$\left(\mathcal{B}_{T} \uparrow c\right) \sqsubseteq_{\mathrm{F}}\left(\mathcal{B}_{T^{\prime}} \uparrow c\right) \wedge \mathcal{C}_{T}=\mathfrak{C}_{T^{\prime}} \wedge \mathcal{C}_{T^{\prime}} \cap \mathfrak{C}_{R}=\emptyset \wedge$

$\left(\left(\mathcal{B}_{T^{\prime}} \uparrow c\right)\left[\left[N_{I O}^{c \rightarrow z}\right]\right]\right)$ is I/O confluent $\wedge$

$\left(\left(\mathcal{B}_{T^{\prime}} \nmid c\right)\left[\left[N_{I O}^{c \rightarrow z}\right]\right]\right) \approx\left(\left(\mathcal{B}_{R}\lceil z)\left[\left[N_{I O}^{z \rightarrow c}\right]\right]\right)\right.$ 
$\Rightarrow\left[\sqsubseteq_{\mathrm{F}}\right.$ preserves finite output property (Lemma 3.7) $\wedge\left(\left(\mathcal{B}_{T} \uparrow c\right)\left[\left[N_{I O}^{c \rightarrow z}\right]\right]\right)$ satisfies FOP $]$

$\left(\mathcal{B}_{T} \uparrow c\right) \sqsubseteq_{\mathrm{F}}\left(\mathcal{B}_{T^{\prime}} \uparrow c\right) \wedge \mathcal{C}_{T}=\mathcal{C}_{T^{\prime}} \wedge \mathcal{C}_{T^{\prime}} \cap \mathcal{C}_{R}=\emptyset \wedge$

$\left(\left(\mathcal{B}_{T^{\prime}} \uparrow c\right)\left[\left[N_{I O}^{c \rightarrow z}\right]\right]\right)$ is I/O confluent $\wedge$

$\left(\left(\mathcal{B}_{T^{\prime}} \uparrow c\right)\left[\left[N_{I O}^{c \rightarrow z}\right]\right]\right) \approx\left(\left(\mathcal{B}_{R} \uparrow z\right)\left[\left[N_{I O}^{z \rightarrow c}\right]\right]\right) \wedge$

$\left(\left(\mathcal{B}_{T^{\prime}} \uparrow c\right)\left[\left[N_{I O}^{c \rightarrow z}\right]\right]\right)$ satisfies FOP

$\Rightarrow\left[\begin{array}{l}\text { deadlock free communication composition (Theorem 2.1) } \\ \text { communication composition (Definition 2.17) }\end{array}\right]$

$\left(\mathcal{B}_{T} \uparrow c\right) \sqsubseteq_{\mathrm{F}}\left(\mathcal{B}_{T^{\prime}} \uparrow c\right) \wedge \mathcal{C}_{T}=\mathcal{C}_{T^{\prime}} \wedge\left(\mathcal{C}_{T^{\prime}} \cap \mathcal{C}_{R}\right)=\emptyset \wedge\left(\left(\mathcal{B}_{T^{\prime}} \uparrow c\right)\left[\left[N_{I O}^{c \rightarrow z}\right]\right]\right)$ is I/O confluent $\wedge$

$\left(\left(\mathcal{B}_{T^{\prime}} \uparrow c\right)\left[\left[N_{I O}^{c \rightarrow z}\right]\right]\right) \approx\left(\left(\mathcal{B}_{R} \uparrow z\right)\left[\left[N_{I O}^{z \rightarrow c}\right]\right]\right) \wedge\left(\left(\mathcal{B}_{T^{\prime}} \uparrow c\right)\left[\left[N_{I O}^{c \rightarrow z}\right]\right]\right)$ satisfies FOP $\wedge$

$(T[c \leftrightarrow z] R)$ and $\left(T^{\prime}[c \leftrightarrow z] R\right)$ are deadlock-free contracts

$\Rightarrow\left[\begin{array}{l}\mathcal{B}_{\left(T^{\prime}[c \leftrightarrow z] R\right)}=\left(\mathcal{B}_{T} \| \mathcal{B}_{R}\right) \|_{\{|c, z|\}} B U F F_{I O}^{\infty}(c, z) \wedge \\ \mathcal{B}_{\left(T^{\prime}[c \leftrightarrow z] R\right)}=\left(\mathcal{B}_{T^{\prime}} \| \mathcal{B}_{R}\right)\|\|_{\{\mid c, z]\}} B U F F_{I O}^{\infty}(c, z) \wedge \\ \text { monotonicity of } \sqsubseteq_{\mathrm{F}}\end{array}\right]$

$\left(\left(\mathcal{B}_{T^{\prime}} \uparrow c\right)\left[\left[N_{I O}^{c \rightarrow z}\right]\right]\right)$ is I/O confluent $\wedge\left(\left(\mathcal{B}_{T^{\prime}} \uparrow c\right)\left[\left[N_{I O}^{c \rightarrow z}\right]\right]\right) \approx\left(\left(\mathcal{B}_{R}\lceil z)\left[\left[N_{I O}^{z \rightarrow c}\right]\right]\right) \wedge\right.$

$\left(\left(\mathcal{B}_{T^{\prime}}^{\uparrow} c\right)\left[\left[N_{I O}^{c \rightarrow z}\right]\right]\right)$ satisfies FOP $\wedge(T[c \leftrightarrow z] R)$ and $\left(T^{\prime}[c \leftrightarrow z] R\right)$ are deadlock-free contracts $\wedge$

$\mathcal{B}_{(T[c \leftrightarrow z] R)} \sqsubseteq_{\mathrm{F}} \mathcal{B}_{\left(T^{\prime}[c \leftrightarrow z] R\right)} \wedge \mathcal{C}_{(T[c \leftrightarrow z] R)}=\mathcal{C}_{\left(T^{\prime}[c \leftrightarrow z] R\right)}$

$\Rightarrow$ [refinement (Definition 3.1) $\wedge$ communication composition (Definition 2.17)]

$(T[c \leftrightarrow z] R) \sqsubseteq_{\mathcal{B}}\left(T^{\prime}[c \leftrightarrow z] R\right)$

The communication composition rule (Definition 2.17) assumes, among other conditions, that the deadlock free components $T$ and $R$ can be hooked by their channels $c$ and $z\left(c \in \mathcal{C}_{T}\right.$ and $\left.z \in \mathcal{C}_{R}\right)$ if $T \nmid c\left[\left[N_{I O}^{c \rightarrow z}\right]\right]$ and $R \nmid z\left[\left[N_{I O}^{z \rightarrow c}\right]\right]$ are I/O confluent, strong compatible and satisfy the finite output property. These conditions are, therefore, required by the previous lemma.

Lemma 3.11 (Feedback composition is monotonic with respect to $\mathcal{B} \mathcal{R J \mathcal { C }}$ refinement). Consider $T$ and $T^{\prime}$ two component contracts, such that $\{c, z\} \subseteq \mathcal{C}_{T}$ are decoupled, $(T \uparrow c)\left[\left[N_{I O}^{c \rightarrow z}\right]\right]$ and $(T \nmid z)\left[\left[N_{I O}^{z \rightarrow c}\right]\right]$ are I/O confluent, strong compatible and satisfy the finite output property. If $T \sqsubseteq_{\mathcal{B}} T^{\prime}$, then $T[c \hookrightarrow z] \sqsubseteq_{\mathcal{B}} T^{\prime}[c \hookrightarrow z]$.

Proof.

$\left[\{c, z\} \subseteq \mathcal{C}_{T}\right.$ are decoupled $\wedge T[c \hookrightarrow z]$ is a $\mathcal{B} \mathcal{R J} \mathcal{C}$ component $\left.\wedge T \sqsubseteq_{\mathcal{B}} T^{\prime}\right]$

$\left(\mathcal{B}_{T} \uparrow c\right) \sqsubseteq_{\mathrm{F}}\left(\mathcal{B}_{T^{\prime}} \uparrow c\right) \wedge\left(\mathcal{B}_{T} \uparrow z\right) \sqsubseteq_{\mathrm{F}}\left(\mathcal{B}_{T^{\prime}} \uparrow z\right) \wedge \mathcal{C}_{T}=\mathcal{C}_{T^{\prime}} \wedge\{c, z\} \subseteq \mathcal{C}_{T^{\prime}}$

$\Rightarrow\left[N_{I O}^{c \rightarrow z}\right.$ and $N_{I O}^{z \rightarrow c}$ are bijections $]$

$\{c, z\} \subseteq \mathcal{C}_{T^{\prime}} \wedge\left(\left(\mathcal{B}_{T}\lceil c)\left[\left[N_{I O}^{c \rightarrow z}\right]\right) \sqsubseteq_{\mathrm{F}}\left(\left(\mathcal{B}_{T^{\prime}} \uparrow c\right)\left[\left[N_{I O}^{c \rightarrow z}\right]\right) \wedge\left(\left(\mathcal{B}_{T} \uparrow z\right)\left[\left[N_{I O}^{z \rightarrow c}\right]\right) \sqsubseteq_{\mathrm{F}}\left(\left(\mathcal{B}_{T^{\prime}} \uparrow z\right)\left[\left[N_{I O}^{z \rightarrow c}\right]\right]\right)\right.\right.\right.\right.$

$\Rightarrow\left[\begin{array}{l}\sqsubseteq_{\mathrm{F}} \text { preserves I/O confluence (Theorem 3.1) } \\ \left(\mathcal{B}_{T} \uparrow c\right)\left[\left[N_{I O}^{c \rightarrow z}\right]\right] \text { and }\left(\mathcal{B}_{T} \uparrow z\right)\left[\left[N_{I O}^{z \rightarrow c}\right]\right] \text { are I/O confluent }\end{array}\right]$ 
$\{c, z\} \subseteq \mathcal{C}_{T^{\prime}} \wedge\left(\left(\mathcal{B}_{T}^{\uparrow} c\right)\left[\left[N_{I O}^{c \rightarrow z}\right]\right]\right) \sqsubseteq_{\mathrm{F}}\left(\left(\mathcal{B}_{T^{\prime}} \uparrow c\right)\left[\left[N_{I O}^{c \rightarrow z}\right]\right]\right) \wedge\left(\left(\mathcal{B}_{T} \uparrow z\right)\left[\left[N_{I O}^{z \rightarrow c}\right]\right) \sqsubseteq_{\mathrm{F}}\left(\left(\mathcal{B}_{T^{\prime}}\lceil z)\left[\left[N_{I O}^{z \rightarrow c}\right]\right]\right)\right.\right.$

$\wedge\left(\left(\mathcal{B}_{T^{\prime}} \uparrow c\right)\left[\left[N_{I O}^{c \rightarrow z}\right]\right]\right)$ and $\left(\left(\mathcal{B}_{T^{\prime}} \uparrow z\right)\left[\left[N_{I O}^{z \rightarrow c}\right]\right]\right)$ are I/O confluent

$\Rightarrow\left[\sqsubseteq_{\mathrm{F}}\right.$ preserves strong protocol compatibility (Lemma 3.6) $\wedge\left(\left(\mathcal{B}_{T} \uparrow c\right)\left[\left[N_{I O}^{c \rightarrow z}\right]\right]\right) \approx\left(\left(\mathcal{B}_{T} \uparrow z\right)\left[\left[N_{I O}^{z \rightarrow c}\right]\right)\right]$

$\{c, z\} \subseteq \mathcal{C}_{T^{\prime}} \wedge\left(\left(\mathcal{B}_{T} \uparrow c\right)\left[\left[N_{I O}^{c \rightarrow z}\right]\right]\right) \sqsubseteq_{\mathrm{F}}\left(\left(\mathcal{B}_{T^{\prime}} \uparrow c\right)\left[\left[N_{I O}^{c \rightarrow z}\right]\right]\right) \wedge\left(\left(\mathcal{B}_{T} \uparrow z\right)\left[\left[N_{I O}^{z \rightarrow c}\right]\right]\right) \sqsubseteq_{\mathrm{F}}\left(\left(\mathcal{B}_{T^{\prime}} \uparrow z\right)\left[\left[N_{I O}^{z \rightarrow c}\right]\right]\right)$

$\wedge\left(\left(\mathcal{B}_{T^{\prime}} \uparrow c\right)\left[\left[N_{I O}^{c \rightarrow z}\right]\right]\right)$ and $\left(\left(\mathcal{B}_{T^{\prime}} \uparrow z\right)\left[\left[N_{I O}^{z \rightarrow c}\right]\right]\right)$ are I/O confluent

$\left.\wedge\left(\left(\mathcal{B}_{T^{\prime}} \uparrow c\right)\left[\left[N_{I O}^{c \rightarrow z}\right]\right]\right) \approx\left(\left(\mathcal{B}_{T^{\prime}} \uparrow z\right)\left[\left[N_{I O}^{z \rightarrow c}\right]\right]\right)\right]$

$\Rightarrow\left[\sqsubseteq\right.$ F preserves FOP $($ Lemma 3.7) $)\left(\left(\mathcal{B}_{T} \uparrow c\right)\left[\left[N_{I O}^{c \rightarrow z}\right]\right]\right)$ and $\left(\left(\mathcal{B}_{T} \uparrow z\right)\left[\left[N_{I O}^{z \rightarrow c}\right]\right]\right)$ satisfy FOP $]$

$\{c, z\} \subseteq \mathcal{C}_{T^{\prime}} \wedge\left(\left(\mathcal{B}_{T} \uparrow c\right)\left[\left[N_{I O}^{c \rightarrow z}\right]\right]\right) \sqsubseteq_{\mathrm{F}}\left(\left(\mathcal{B}_{T^{\prime}} \uparrow c\right)\left[\left[N_{I O}^{c \rightarrow z}\right]\right]\right) \wedge\left(\left(\mathcal{B}_{T} \uparrow z\right)\left[\left[N_{I O}^{z \rightarrow c}\right]\right]\right) \sqsubseteq_{\mathrm{F}}\left(\left(\mathcal{B}_{T^{\prime}} \uparrow z\right)\left[\left[N_{I O}^{z \rightarrow c}\right]\right]\right)$

$\wedge\left(\left(\mathcal{B}_{T^{\prime}} \uparrow c\right)\left[\left[N_{I O}^{c \rightarrow z}\right]\right]\right)$ and $\left(\left(\mathcal{B}_{T^{\prime}} \uparrow z\right)\left[\left[N_{I O}^{z \rightarrow c}\right]\right]\right)$ are I/O confluent

$\left.\wedge\left(\left(\mathcal{B}_{T^{\prime}} \uparrow c\right)\left[\left[N_{I O}^{c \rightarrow z}\right]\right]\right) \approx\left(\left(\mathcal{B}_{T^{\prime}} \uparrow z\right)\left[\left[N_{I O}^{z \rightarrow c}\right]\right]\right)\right]$

$\wedge\left(\left(\mathcal{B}_{T^{\prime}} \uparrow c\right)\left[\left[N_{I O}^{c \rightarrow z}\right]\right]\right)$ and $\left(\left(\mathcal{B}_{T^{\prime}} \uparrow z\right)\left[\left[N_{I O}^{z \rightarrow c}\right]\right]\right)$ satisfies FOP

$\Rightarrow[\mathcal{B R J C}$ refinement preserves decoupled channels (Lemma 3.8) $\wedge\{c, z\}$ are decoupled in $T]$

$\{c, z\} \subseteq \mathcal{C}_{T^{\prime}} \wedge\left(\left(\mathcal{B}_{T} \uparrow c\right)\left[\left[N_{I O}^{c \rightarrow z}\right]\right]\right) \sqsubseteq_{\mathrm{F}}\left(\left(\mathcal{B}_{T^{\prime}} \uparrow c\right)\left[\left[N_{I O}^{c \rightarrow z}\right]\right]\right) \wedge\left(\left(\mathcal{B}_{T} \uparrow z\right)\left[\left[N_{I O}^{z \rightarrow c}\right]\right) \sqsubseteq_{\mathrm{F}}\left(\left(\mathcal{B}_{T^{\prime}} \uparrow z\right)\left[\left[N_{I O}^{z \rightarrow c}\right]\right]\right)\right.$

$\wedge\left(\left(\mathcal{B}_{T^{\prime}} \uparrow c\right)\left[\left[N_{I O}^{c \rightarrow z}\right]\right]\right)$ and $\left(\left(\mathcal{B}_{T^{\prime}} \uparrow z\right)\left[\left[N_{I O}^{z \rightarrow c}\right]\right]\right)$ are I/O confluent

$\left.\wedge\left(\left(\mathcal{B}_{T^{\prime}} \uparrow c\right)\left[\left[N_{I O}^{c \rightarrow z}\right]\right]\right) \approx\left(\left(\mathcal{B}_{T^{\prime}} \uparrow z\right)\left[\left[N_{I O}^{z \rightarrow c}\right]\right]\right)\right]$

$\wedge\left(\left(\mathcal{B}_{T^{\prime}} \uparrow c\right)\left[\left[N_{I O}^{c \rightarrow z}\right]\right]\right)$ and $\left(\left(\mathcal{B}_{T^{\prime}} \uparrow z\right)\left[\left[N_{I O}^{z \rightarrow c}\right]\right]\right)$ satisfies FOP

$\wedge\{c, z\}$ are decoupled in $T^{\prime}$

$\Rightarrow$ [deadlock free feedback composition (Theorem 2.1) $\wedge$ feedback composition (Definition 2.18) ]

$\{c, z\} \subseteq \mathcal{C}_{T^{\prime}} \wedge\left(\left(\mathcal{B}_{T^{\prime}} \uparrow c\right)\left[\left[N_{I O}^{c \rightarrow z}\right]\right]\right)$ and $\left(\left(\mathcal{B}_{T^{\prime}} \uparrow z\right)\left[\left[N_{I O}^{z \rightarrow c}\right]\right]\right)$ are I/O confluent

$\left.\wedge\left(\left(\mathcal{B}_{T^{\prime}} \uparrow c\right)\left[\left[N_{I O}^{c \rightarrow z}\right]\right]\right) \approx\left(\left(\mathcal{B}_{T^{\prime}} \uparrow z\right)\left[\left[N_{I O}^{z \rightarrow c}\right]\right]\right)\right]$

$\wedge\left(\left(\mathcal{B}_{T^{\prime}} \uparrow c\right)\left[\left[N_{I O}^{c \rightarrow z}\right]\right]\right)$ and $\left(\left(\mathcal{B}_{T^{\prime}} \uparrow z\right)\left[\left[N_{I O}^{z \rightarrow c}\right]\right]\right)$ satisfies FOP

$\wedge\{c, z\}$ are decoupled in $T^{\prime} \wedge(T[c \hookrightarrow z])$ and $\left(T^{\prime}[c \hookrightarrow z]\right)$ are deadlock-free contracts

$\Rightarrow\left[\begin{array}{l}\mathcal{B}_{(T[c \hookrightarrow z])}=\mathcal{B}_{T} \|_{\{\mid c, z]\}} B U F F_{I O}^{\infty}(c, z) \wedge \\ \mathcal{B}_{\left(T^{\prime}[c \hookrightarrow z]\right)}=\mathcal{B}_{T^{\prime}} \|_{\{c, z\}\}} B U F F_{I O}^{\infty}(c, z) \wedge \\ \text { monotonicity of } \sqsubseteq_{\mathrm{F}}\end{array}\right]$

$\{c, z\} \subseteq \mathcal{C}_{T^{\prime}} \wedge\left(\left(\mathcal{B}_{T^{\prime}} \uparrow c\right)\left[\left[N_{I O}^{c \rightarrow z}\right]\right]\right)$ and $\left(\left(\mathcal{B}_{T^{\prime}} \uparrow z\right)\left[\left[N_{I O}^{z \rightarrow c}\right]\right]\right)$ are I/O confluent

$\left.\wedge\left(\left(\mathcal{B}_{T^{\prime}} \uparrow c\right)\left[\left[N_{I O}^{c \rightarrow z}\right]\right]\right) \approx\left(\left(\mathcal{B}_{T^{\prime}} \uparrow z\right)\left[\left[N_{I O}^{z \rightarrow c}\right]\right]\right)\right]$

$\wedge\left(\left(\mathcal{B}_{T^{\prime}} \uparrow c\right)\left[\left[N_{I O}^{c \rightarrow z}\right]\right]\right)$ and $\left(\left(\mathcal{B}_{T^{\prime}} \uparrow z\right)\left[\left[N_{I O}^{z \rightarrow c}\right]\right]\right)$ satisfies FOP

$\wedge\{c, z\}$ are decoupled in $T^{\prime} \wedge(T[c \hookrightarrow z])$ and $\left(T^{\prime}[c \hookrightarrow z]\right)$ are deadlock-free contracts

$\wedge \mathcal{B}_{(T[c \hookrightarrow z])} \sqsubseteq_{\mathrm{F}} \mathcal{B}_{\left(T^{\prime}[c \hookrightarrow z]\right)} \wedge \mathcal{C}_{(T[c \hookrightarrow z])}=\mathcal{C}_{\left(T^{\prime}[c \hookrightarrow z]\right)}$

$\Rightarrow$ [refinement (Definition 3.1) $\wedge$ feedback composition (Definition 2.18)]

$T[c \hookrightarrow z] \sqsubseteq_{\mathcal{B}} T^{\prime}[c \hookrightarrow z]$

To make an internal connection between two channels $c$ and $z$ of a deadlock free compo- 
nent $T$ we need, according to Definition 2.18, to ensure that $(T \nmid c)\left[\left[N_{I O}^{c \rightarrow z}\right]\right]$ and $(T \nmid z)\left[\left[N_{I O}^{z \rightarrow c}\right]\right]$ are I/O confluent, strong compatible and satisfy the finite output property; additionally, $\{c, z\}$ must be decoupled in $T$. These conditions are necessary in the previous lemma because they are preserved by failures refinement $\left(\Xi_{\mathrm{F}}\right)$, which is the basis of $\mathcal{B} \mathcal{R J \mathcal { C }}$ refinement $\left(\sqsubseteq_{\mathcal{B}}\right)$.

Lemma 3.12 (Reflexive composition is monotonic with respect to $\mathcal{B} \mathcal{R J} \mathcal{C}$ refinement). Consider $T$ and $T^{\prime}$ two component contracts, such that $\{c, z\} \subseteq \mathcal{C}_{T}$ and $T \uparrow\{c, z\}$ is buffering self-injection compatible and satisfies the finite output property. If $T \sqsubseteq_{\mathcal{B}} T^{\prime}$, then $T\left[c \stackrel{\hookrightarrow}{\hookrightarrow} z \sqsubseteq_{\mathcal{B}} T^{\prime}[c \stackrel{\hookrightarrow}{\hookrightarrow} z]\right.$.

Proof.

$\left[\{c, z\} \subseteq \mathcal{C}_{T} \wedge T\left[c^{\hookrightarrow} \rightrightarrows z\right]\right.$ is a $\mathcal{B R J C}$ component $\left.\wedge T \sqsubseteq_{\mathcal{B}} T^{\prime}\right]$

$\left(\mathcal{B}_{T} \uparrow\{|c, z|\}\right) \sqsubseteq_{\mathrm{F}}\left(\mathcal{B}_{T^{\prime}} \uparrow\{|c, z|\}\right) \wedge \mathcal{C}_{T}=\mathcal{C}_{T^{\prime}} \wedge\{c, z\} \subseteq \mathcal{C}_{T^{\prime}}$

$\Rightarrow\left[\begin{array}{l}\sqsubseteq_{\mathrm{F}} \text { preserves self-injection compatibility (Theorem 3.2) } \\ \left(\mathcal{B}_{T} \uparrow\{|c, z|\}\right) \text { is buffering self injection compatible }\end{array}\right]$

$\left(\mathcal{B}_{T} \uparrow\{|c, z|\}\right) \sqsubseteq_{\mathrm{F}}\left(\mathcal{B}_{T^{\prime}} \uparrow\{|c, z|\}\right) \wedge\{c, z\} \subseteq \mathcal{C}_{T^{\prime}} \wedge$

$\left(\mathcal{B}_{T^{\prime}} \uparrow\{|c, z|\}\right)$ is buffering self injection compatible

$\Rightarrow\left[\sqsubseteq_{\mathrm{F}}\right.$ preserves FOP $\left(\right.$ Lemma 3.7) $\wedge\left(\mathcal{B}_{T} \uparrow\{|c, z|\}\right)$ satisfies FOP $]$

$\left(\mathcal{B}_{T} \uparrow\{|c, z|\}\right) \sqsubseteq_{\mathrm{F}}\left(\mathcal{B}_{T^{\prime}} \uparrow\{|c, z|\}\right) \wedge\{c, z\} \subseteq \mathcal{C}_{T^{\prime}} \wedge$

$\left(\mathcal{B}_{T^{\prime}} \uparrow\{|c, z|\}\right)$ is buffering self injection compatible $\wedge\left(\mathcal{B}_{T^{\prime}} \uparrow\{|c, z|\}\right)$ satisfies FOP

$\Rightarrow\left[\begin{array}{l}\text { deadlock free reflexive composition (Theorem 2.1) } \\ \text { reflexive composition (Definition 2.19) }\end{array}\right]$

$\left(\mathcal{B}_{T} \uparrow\{|c, z|\}\right) \sqsubseteq_{\mathrm{F}}\left(\mathcal{B}_{T^{\prime}} \uparrow\{|c, z|\}\right) \wedge\{c, z\} \subseteq \mathcal{C}_{T^{\prime}} \wedge$

$\left(\mathcal{B}_{T^{\prime}} \uparrow\{|c, z|\}\right)$ is buffering self injection compatible $\wedge$

$\left(\mathcal{B}_{T^{\prime}} \uparrow\{|c, z|\}\right)$ satisfies FOP $\wedge$

$\left(T\left[c^{\hookrightarrow} \vec{\hookrightarrow}\right]\right)$ and $\left(T^{\prime}\left[c^{\hookrightarrow} \rightrightarrows z\right]\right)$ are deadlock-free contracts

$\Rightarrow\left[\begin{array}{cc}\mathcal{B}_{(T[c \hookrightarrow z])}=\mathcal{B}_{T} \|_{\{\mid c, z]\}} B U F F_{I O}^{\infty}(c, z) \wedge \\ \mathcal{B}_{\left(T^{\prime}[c \hookrightarrow z]\right)}=\mathcal{B}_{T^{\prime}} \|_{\{\mid c, z]\}} B U F F_{I O}^{\infty}(c, z) \wedge \\ \text { monotonicity of } \sqsubseteq_{\mathrm{F}}\end{array}\right]$

$\{c, z\} \subseteq \mathcal{C}_{T^{\prime}} \wedge\left(\mathcal{B}_{T^{\prime}} \uparrow\{|c, z|\}\right)$ is buffering self injection compatible $\wedge$

$\left(\mathcal{B}_{T^{\prime}} \uparrow\{|c, z|\}\right)$ satisfies FOP $\wedge$

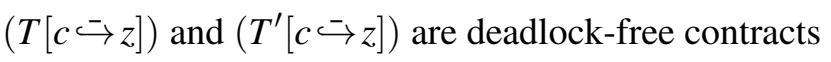

$\wedge \mathcal{B}_{T[c \hookrightarrow z]} \sqsubseteq \mathrm{F} \mathcal{B}_{T^{\prime}[c \hookrightarrow z]} \wedge \mathcal{C}_{T[c \hookrightarrow z]}=\mathcal{C}_{T^{\prime}[c \hookrightarrow z]}$

$\Rightarrow$ [refinement (Definition 3.1) $\wedge$ reflexive composition (Definition 2.19)]

$T[c \stackrel{\hookrightarrow}{\hookrightarrow}] \sqsubseteq_{\mathcal{B}} T^{\prime}[c \stackrel{-}{\hookrightarrow} z]$

A more general rule to hook two channels $c$ and $z$ inside a deadlock-free component $T$ 
is given by the reflexive composition rule (Definition 2.19). From this composition rule, the previous lemma requires $T \nmid\{c, z\}$ to be buffering self-injection compatible and to satisfy the finite output property.

Theorem 3.3 (BRJC composition is monotonic with respect to refinement). Consider $C$ a context (composite component) where BRJC components were assembled by interleaving, communication, feedback and reflexive composition rules. $C[T]$ denotes a context where the component $T$ is used. If $C[T]$ and $T$ are deadlock free contracts and $T \sqsubseteq_{\mathcal{B}} T^{\prime}$ then $C[T] \sqsubseteq_{\mathcal{B}} C\left[T^{\prime}\right]$ and $C\left[T^{\prime}\right]$ is a deadlock-free context.

Proof. It follows directly from Lemmas 3.9, 3.10, 3.11 and 3.12, where the pre-conditions respected by $T$ in the compositions inside the context $C$ are also satisfied by $T^{\prime}$, according to Definition 3.1.

We have demonstrated the monotonicity of the $\mathcal{B} \mathcal{R J C}$ composition operators with respect to refinement. This is an important result since it paves the way for the step- and component-wise evolution of $\mathcal{B R J \mathcal { C }}$ specifications, by the improvement of existing services but, nevertheless, we are not able to define new ones. This is a well know limitation of refinement, since, in general, new services imply in new events which augment the interfaces of existing components; this has motivated the study conducted in the next chapter: component inheritance relations and their connection with refinement. 


\section{4}

\section{BRIC inheritance based on convergence}

In this chapter we present the main contributions of this work: the development of inheritance relations for the $\mathcal{B} \mathcal{R J C}$ component model that supports evolution based on a set of composition rules and the modelling of systems behavioural and structural views, which is based on our results presented in (DIHEGO; SAMPAIO; OLIVEIRA, 2015; DIHEGO, 2015). Our relations are defined on top of a behavioural relation called convergence. It captures the idea that components can evolve by accepting new inputs or establishing a communication session after them but they are able to converge to the predicted behaviour exhibited by its abstractions. This is a concept that cannot be captured only by using abstraction (like that provided by the hiding operator) as can be found in other inheritance relations (WEHRHEIM, 2003).

The concept of inheritance in the object-oriented paradigm is a well known field with a comprehensive literature (LISKOV; WING, 1994). Recently, efforts have been made to extend this concept to process algebras as CSP. Notably, in (WEHRHEIM, 2003) the author proposes four types of behavioural inheritance relations using CSP-equivalent Labelled Transition Systems (LTS). Although very promising, these relations do not consider specifications that distinguish inputs from outputs, such as $\mathcal{B R J \mathcal { C }}$, in which behaviour is modelled as CSP I/O processes. Since I/O processes (Definition 2.4) must satisfy behaviour restrictions such as input determinism and strong output decisiveness, we need relations that can handle these restrictions. We base our approach on a concept of convergence: a convergent process is allowed to do the same or more inputs than its parent process, but is restricted to do the same or less outputs in convergent points. A convergent point represents a state reachable by both the original and the convergent process when doing two convergent sequences of events; these sequences differ only because the convergent process is allowed to do extra inputs (inputs not allowed by the original process) in converging points (see the motivating example in Section 2.2). First, we formalise the concept of convergent traces as follows. 


\section{I/O convergence}

In Definition 4.1 and others that follow, recall that: $\Sigma$ stands for the alphabet of all possible events, $\Sigma^{*}$ is the set of possible sequences of events from $\Sigma$, the input events are contained in $\Sigma$ (inputs $\subseteq \Sigma$ ) and $i n(T, t)$ is a function that yields the set of input events that can be communicated by the I/O process $T$ after some $t \in \mathcal{T}(T)$; therefore in has type I/OProcess $\times \Sigma^{*} \rightarrow$ inputs. Additionally if $t_{1} \leq t_{2}$, it means that $t_{1}$ is a subtrace of $t_{2}$.

A trace $t^{\prime}$ is I/O convergent to $t$ if they are equal or if $t=\mathbf{t}_{\mathbf{0}} \wedge t_{n}$ and $t^{\prime}=\mathbf{t}_{\mathbf{0}} \wedge\langle n e\rangle^{\wedge} t_{n}^{\prime}$ such that $n e \in \operatorname{in}\left(T^{\prime}, t_{0}\right)$ but $n e \notin i n\left(T, t_{0}\right)$ and $t$ is I/O convergent to $t_{0} \wedge t_{n}^{\prime}$. This recursive definition means that $t^{\prime}$ and $t$ differ because $T^{\prime}$ can do new-in-context inputs (inputs not allowed by $T$ ) where $T$ cannot, but despite that the trace $t^{\prime}$ of $T^{\prime}$ has always a counterpart $t$ of $T$. We highlight that these traces differ because of new-in-context inputs performed by $T^{\prime}$; such inputs can belong to the alphabet of $T$, but in $T^{\prime}$ they can appear in different contexts (such as up and down events after sett on TV_RC' , see Section 2.2). Also, because the CSP hiding operator renames events to $\tau$, no matter their context inside a process, it is not a suitable alternative to define convergence, where events have different meanings depending on the place they are communicated.

Definition 4.1 (I/O convergent traces). Consider two I/O processes $T$ and $T^{\prime}$. Let $t$ and $t^{\prime}$ be two respective traces. We say that $t^{\prime}$ is an $I / O$ convergent trace of $t\left(t^{\prime} \operatorname{cvg} t\right)$ if, and only if:

$$
\left.\left(t^{\prime}=t\right) \vee\left(\begin{array}{c}
\left(\# t^{\prime}>\# t\right) \wedge \exists t_{1}, t_{3}: \Sigma^{*}, \exists n e: \Sigma \mid \\
t^{\prime}=t_{1} \wedge\langle n e\rangle^{\wedge} t_{3} \wedge t_{1} \leq t \wedge \\
n e \in \text { inputs } \wedge n e \notin i n\left(T, t_{1}\right) \wedge \\
t_{1} \wedge t_{3} \operatorname{cvg} t
\end{array}\right)\right)
$$

Based on the definition of convergent traces, we are now able to define behavioural convergence.

Definition 4.2 (I/O convergent behaviour). Consider two $I / O$ process $T$ and $T^{\prime} . T^{\prime}$ is an I/O convergent behaviour of $T$ ( $T^{\prime}$ io_cvg $T$ ) if, and only if:

$$
\forall\left(t^{\prime}, X\right) \in \mathcal{F}\left(T^{\prime}\right), \exists(t, Y) \in \mathcal{F}(T) \bullet\left(\begin{array}{c}
t^{\prime} \operatorname{cvg} t \wedge \\
Y \cap \text { inputs } \supseteq X \cap \text { inputs } \wedge \\
Y \cap \text { outputs } \subseteq X \cap \text { outputs }
\end{array}\right)
$$

An I/O process is convergent to another if in each converging point of their execution $\left(t^{\prime} \operatorname{cvg} t\right)$ it can offer more or equal inputs ( $Y \cap$ inputs $\supseteq X \cap$ inputs) but is restricted to offer less or equal outputs ( $Y \cap$ out puts $\subseteq X \cap$ out puts). A convergent point relates two I/O processes stable states: one in $T$ after it communicates $t$ and, another in $T^{\prime}$ after it communicates $t^{\prime}$, such that $\left(t^{\prime} \operatorname{cvg} t\right)$. In what follows, we illustrate this definition. 
Figure 4.1: I/O convergent behaviours
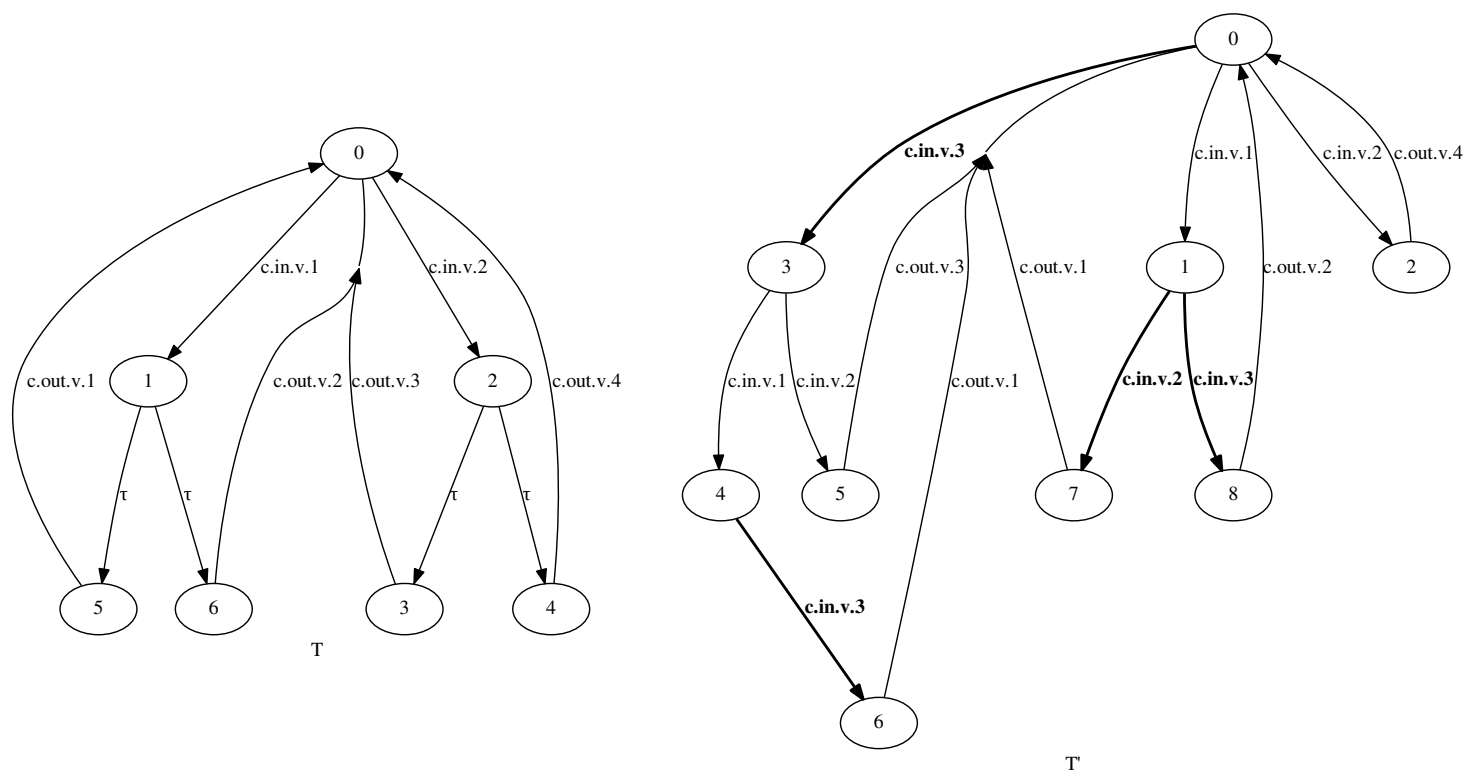

Source: José Dihego

Example 4.1 (I/O convergent behaviour).

Let us consider the I/O processes $T$ and $T^{\prime}$ (Figure 4.1). Based on Definition 4.2, we have that $T^{\prime}$ io_cvg $T$. To explain why this is the case, let $\left(t^{\prime}, X\right)$ and $(t, Y)$ be failures of $T^{\prime}$ and $T$, respectively, then:

- $\left(t^{\prime}, X\right)=(\langle$ c.in.v.3, c.in.v.1,c.in.v. 3$\rangle,\{|c|\} \backslash\{$ c.out.v. 1$\})$, then it is possible to find $(t, Y)=(\langle c . i n . v .1\rangle,\{|c|\} \backslash\{c . o u t . v .1\})$, and trivially $t^{\prime} \operatorname{cvg} t, Y \cap$ inputs $=X \cap$ inputs $=$ $\{|c . i n|\}$ and $Y \cap$ outputs $=X \cap$ outputs $=\{\mid$ c.out $\mid\} \backslash\{$ c.out.v. 1$\}$;

- $\left(t^{\prime}, X\right)=(\langle$ c.in.v. 1$\rangle,\{|c|\} \backslash\{c . i n . v \cdot 2$, c.in.v. 3$\})$, then it is possible to find $(t, Y)=$ $(\langle c . i n . v .1\rangle,\{|c|\} \backslash\{c . o u t . v .2\})$, and trivially $t^{\prime} \operatorname{cvg} t, X \cap$ inputs $=\{|c . i n|\} \backslash\{c . i n . v .2$, c.in.v. 3$\}, X \cap$ out puts $=\{\mid$ c.out $\mid\}, Y \cap$ inputs $=\{|c . i n|\}$ and $Y \cap$ out puts $=\{\mid$ c.out $\mid\}$ । $\{$ c.out.v. 2$\}$.

In Figure 4.1, we boldface input events (and their edges) that are new in context: they are accepted in some states of $T^{\prime}$, but refused on equivalent states of $T$, where equivalence is given by convergence.

Now consider processes $U$ and $U^{\prime}$ in Figure 4.2. By Definition 4.2, $U^{\prime}$ is not an I/O convergent behaviour of $U$ : after communicating c.in.v.3 it goes to State 3 (convergent with State 0 in $U), U^{\prime}$ fails to offer the expected inputs in State 0 in $U$ (c.in.v.1 and c.in.v.2) after the new-in-context input event $c . i n . v .3$ has been communicated. Initially, this is a not a problem for the existing $U$ clients, which can communicate with $U^{\prime}$ provided they do not exercise the behaviour trigged by c.in.v.3, but if a $U^{\prime}$ client is involved, then it can communicate the event 
Figure 4.2: I/O non-convergent behaviour
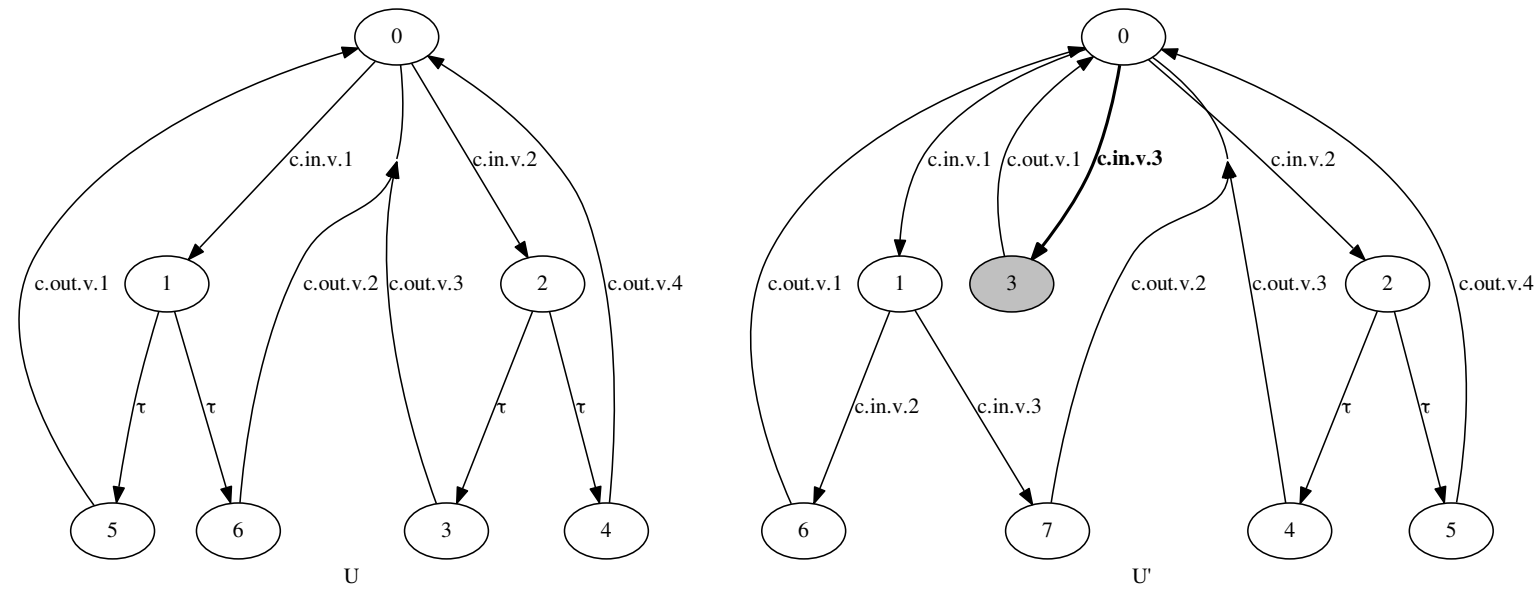

Source: José Dihego

c.in.v.3 leading to an unexpected behaviour from the $U$ clients perspective; therefore, it is not safe to compose $U$ and $U^{\prime}$ clients with a $U^{\prime} \mathrm{I} / \mathrm{O}$ process. We can prove $U^{\prime}$ is not I/O convergent to $U$ by analysing a failure $\left(t^{\prime}, X\right) \in \mathcal{F}\left(U^{\prime}\right)$, where $\left(t^{\prime}, X\right)=(\langle$ c.in.v. 3$\rangle,\{|c|\} \backslash\{$ c.out.v. 1$\})$. The unique convergent trace to $t^{\prime}$ in $\mathcal{T}(U)$ is the empty trace, which raises the unique failure pair $(t, Y)=(\langle\rangle,\{|c|\} \backslash\{c . i n . v .1, c . i n . v .2\}) \in \mathcal{F}(U)$. Therefore, $X \cap$ inputs $=\{|c . i n|\}$ and $Y \cap$ inputs $=$ $\{|c . i n|\} \backslash\{$ c.in.v.1,c.in.v.2\} which does not satisfy the condition of Definition 4.2. Note that State 3 is drawn in gray to highlight the point where the inputs expected by $T$ after trace $\langle$ c.in.v. 3$\rangle$ were not offered.

\section{I/O extended convergence}

A convergent I/O process can engage in more inputs to take more deterministic decisions on what to output. Nevertheless, it can be useful to offer other events after a new input and before converging to its counterpart according to the relation. This extension to convergence allows convergent processes to add more implementation details. We define this relation in the traces and failures behavioural models in Definitions 4.3 and 4.4, respectively.

Definition 4.3 (I/O extended convergent traces). Consider two I/O processes $T$ and $T^{\prime}$. Let $t$ and $t^{\prime}$ be two of their traces, respectively. We say that $t^{\prime}$ is an I/O extended convergent trace of $t$ $\left(t^{\prime} \operatorname{ecvg} t\right)$ if and only if:

$$
\left(t^{\prime}=t\right) \vee\left(\begin{array}{c}
\left(\# t^{\prime}>\# t\right) \wedge \exists t_{1}, t_{2}, t_{3}: \Sigma^{*}, \exists n e \in \Sigma \mid \\
t^{\prime}=t_{1} \wedge\langle n e\rangle^{\wedge} t_{2} \wedge t_{3} \wedge t_{1} \leq t \wedge \\
n e \in \text { inputs } \wedge n e \notin \operatorname{in}\left(T, t_{1}\right) \wedge \\
\operatorname{set}\left(t_{2}\right) \cap\left(\text { in }\left(T, t_{1}\right) \cup \operatorname{out}\left(T, t_{1}\right)\right)=\emptyset \wedge \\
t_{1} \wedge t_{3} \operatorname{ecvg} t
\end{array}\right)
$$


A trace $t^{\prime}$ is I/O extended convergent to $t$ if they are the same or if its possible to equate them by concealing each event $n e$ that is possible for $T^{\prime}$ but not for $T$ after a common subtrace, say $t_{1}$, of $t$ and $t^{\prime}$ (ne $\notin i n\left(T, t_{1}\right)$, but $n e \in i n\left(T^{\prime}, t_{1}\right)$ ); furthermore, since we allow more events after a new input $n e$ we also conceal them $\left(\operatorname{set}\left(t_{2}\right) \cap\left(\operatorname{in}\left(T, t_{1}\right) \cup\right.\right.$ out $\left.\left.\left(T, t_{1}\right)\right)=\emptyset\right)$.

Definition 4.4 (I/O extended convergent behaviour). Consider two I/O process $T$ and $T^{\prime}$. We say that $T^{\prime}$ is an I/O extended convergent behaviour of $T$ ( $T^{\prime}$ io_ecvg $T$ ), if and only if:

$$
\left.\forall\left(t^{\prime}, X\right) \in \mathcal{F}\left(T^{\prime}\right), \exists(t, Y) \in \mathcal{F}(T) \bullet t^{\prime} \operatorname{ecvg} t \wedge\left(\begin{array}{c}
Y \cap \text { inputs } \supseteq X \cap \text { inputs } \wedge \\
Y \cap \text { out puts } \subseteq X \cap \text { outputs }
\end{array}\right)\right)
$$

Definition 4.4 is very similar to Definition 4.2, but allows the extended convergent process $T^{\prime}$ to accept any event not expected by $T(\Sigma \backslash Y \subseteq X)$ in an extended convergent point of their execution $\left(t^{\prime} \operatorname{ecvg} t\right)$, provided a new input $n e$ (see Definition 4.3) has happened, marking the start of the extended convergent behaviour of $T^{\prime}$. We illustrate this definition with an example.

Example 4.2 (I/O extended convergent behaviour).

Consider the processes $T$ and $T^{\prime}$ (Figure 4.3), where $T^{\prime}$ io_ecvg $T$. To gather some evidence that this is the case, we analyse a couple of failures of these processes. Assume that $\left(t^{\prime}, X\right) \in \mathcal{F}\left(T^{\prime}\right)$ and $(t, Y) \in \mathcal{F}(T)$, then if:

- $\left(t^{\prime}, X\right)=(\langle$ c.in.v.3, c.in.v. 4$\rangle,\{|c|\} \backslash\{$ c.out.v. 1$\})$, we can find $(t, Y)=(\langle\rangle,\{|c|\} \backslash\{$ c.in. v.1,c.in.v.2\}), such that $t^{\prime} \operatorname{ecvg} t, \Sigma \backslash Y=\{$ c.in.v.1,c.in.v.2 $\}$ and, therefore $\Sigma \backslash Y \subseteq X$;

- $\left(t^{\prime}, X\right)=(\langle$ c.in.v.3, c.in.v. 4$\rangle,\{|c|\} \backslash\{$ c.out.v. 2$\})$, we can find $(t, Y)=(\langle\rangle,\{|c|\} \backslash\{$ c.in. v.1,c.in.v.2\}), such that $t^{\prime} \operatorname{ecvg} t, \Sigma \mid Y=\{c . i n . v .1, c . i n . v .2\}$ and, therefore $\Sigma \mid Y \subseteq X$;

- $\left(t^{\prime}, X\right)=(\langle$ c.in.v.1, c.in.v. 3$\rangle,\{|c|\} \backslash\{$ c.out.v. 2$\})$, we can find $(t, Y)=(\langle$ c.in.v. 1$\rangle,\{|c|\}$ I $\{$ c.out.v. 2$\})$, such that $t^{\prime} \operatorname{ecvg} t, X=Y$ and, therefore Definition 4.4 holds;

- $\left(t^{\prime}, X\right)=(\langle$ c.in.v. 2$\rangle,\{|c|\} \backslash\{$ c.out.v. 4$\})$, we can find $(t, Y)=(\langle$ c.in.v. 2$\rangle,\{|c|\} \backslash\{$ c.out. $v .4\})$ and trivially Definition 4.4 holds.

As we expect, extended convergence is a generalisation of convergence. Lemmas 4.1 and 4.2 prove this fact.

Lemma 4.1 (cvg $\subseteq$ ecvg). Consider two I/O processes $T$ and $T^{\prime}$, and $t$ and $t^{\prime}$ two of its traces, respectively $\left(t \in \mathcal{T}(T)\right.$ and $\left.t^{\prime} \in \mathcal{T}\left(T^{\prime}\right)\right)$. If $t^{\prime} \operatorname{cvg} t$ then $t^{\prime} \operatorname{ecvg} t$. 
Figure 4.3: I/O extended convergent behaviours

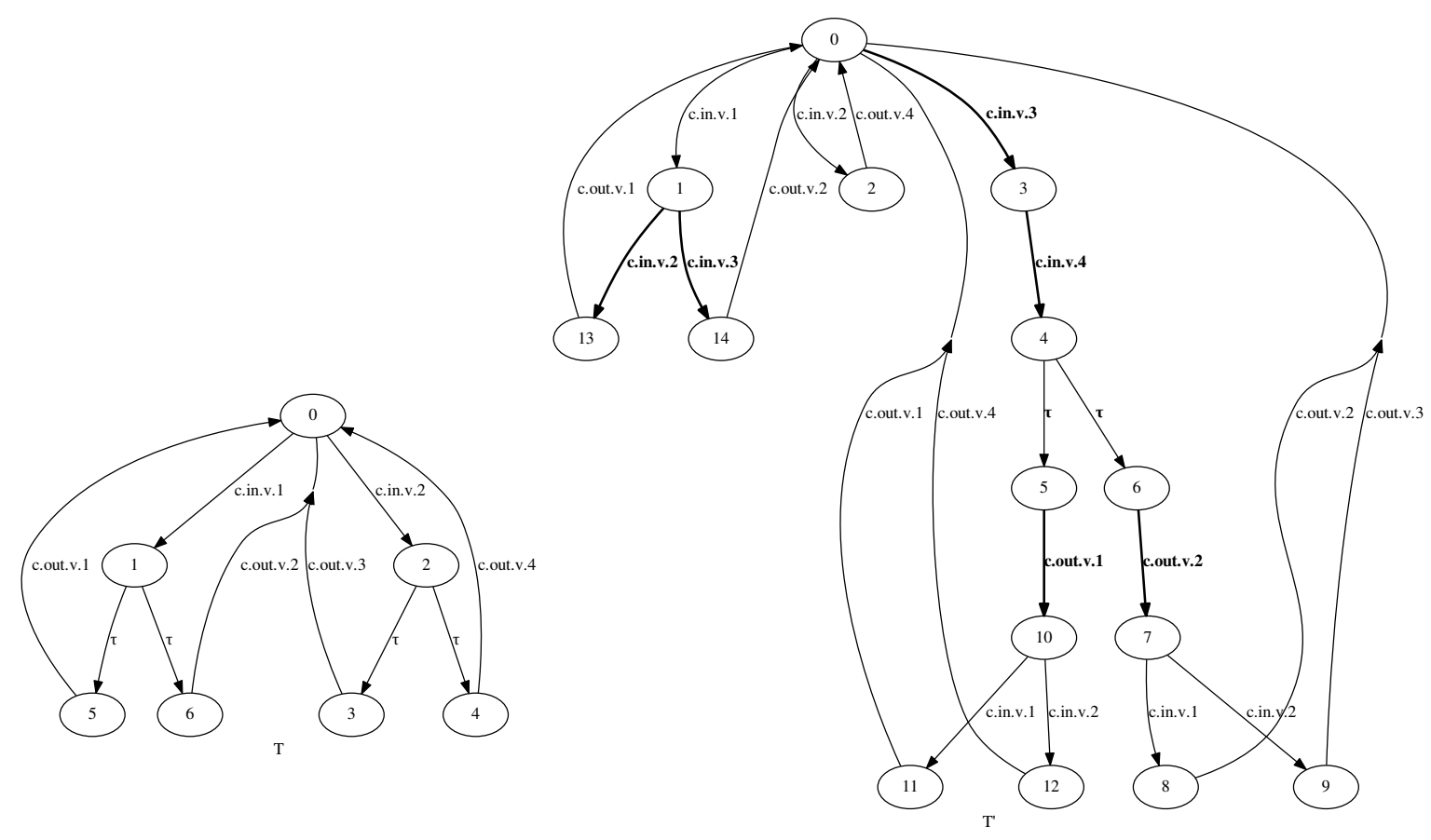

Source: José Dihego

Proof.

$\left[t^{\prime} \operatorname{cvg} t\right]$

$$
\begin{aligned}
& \left(t^{\prime}=t\right) \vee\left(\begin{array}{c}
\left(\# t^{\prime}>\# t\right) \wedge \exists t_{1}, t_{3}: \Sigma^{*}, \exists n e: \Sigma \mid \\
t^{\prime}=t_{1} \wedge\langle n e\rangle^{\wedge} t_{3} \wedge t_{1} \leq t \wedge \\
n e \in \text { inputs } \wedge n e \notin i n\left(T, t_{1}\right) \wedge \\
t_{1} \wedge t_{3} \operatorname{cvg} t
\end{array}\right) \\
& \Rightarrow\left[t_{2}=\langle\rangle\right] \\
& \left(t^{\prime}=t\right) \vee\left(\begin{array}{c}
\left(\# t^{\prime}>\# t\right) \wedge \exists t_{1}, t_{3}: \Sigma^{*}, \exists n e \in \Sigma \mid \\
t^{\prime}=t_{1} \wedge\langle n e\rangle^{\wedge} t_{2} \wedge t_{3} \wedge t_{1} \leq t \wedge \\
n e \in \text { inputs } \wedge n e \notin \text { in }\left(T, t_{1}\right) \wedge \\
\operatorname{set}\left(t_{2}\right) \cap\left(\text { in }\left(T, t_{1}\right) \cup \operatorname{out}\left(T, t_{1}\right)\right)=\emptyset \wedge \\
t_{1} \wedge t_{3} \mathrm{cvg} t
\end{array}\right)
\end{aligned}
$$

$\Rightarrow$ [induction over the predicate $\left.t_{1} \wedge t_{3} \operatorname{cvg} t \mid t_{2}=\langle\rangle\right]$

$\left(t^{\prime}=t\right) \vee\left(\begin{array}{c}\left(\# t^{\prime}>\# t\right) \wedge \exists t_{1}, t_{3}: \Sigma^{*}, \exists n e \in \Sigma \mid \\ t^{\prime}=t_{1} \wedge\langle n e\rangle^{\wedge} t_{2} \wedge t_{3} \wedge t_{1} \leq t \wedge \\ n e \in \text { inputs } \wedge n e \notin \text { in }\left(T, t_{1}\right) \wedge \\ \operatorname{set}\left(t_{2}\right) \cap\left(\text { in }\left(T, t_{1}\right) \cup \operatorname{out}\left(T, t_{1}\right)\right)=\emptyset \wedge \\ t_{1} \wedge t_{3} \operatorname{ecvg} t\end{array}\right)$

$\Rightarrow[\mathrm{I} / \mathrm{O}$ extended convergent traces (Definition 4.3)]

$t^{\prime} \operatorname{ecvg} t$ 
Lemma 4.2 (io_cvg $\subseteq$ io_ecvg). Consider two $I / O$ processes $T$ and $T^{\prime}$. If $T^{\prime}$ io_cvg $T$ then $T^{\prime}$ io_ecvg $T$.

Proof.

$$
\begin{aligned}
& {\left[T^{\prime} \text { io_cvg } T\right]} \\
& \forall\left(t^{\prime}, X\right) \in \mathcal{F}\left(T^{\prime}\right), \exists(t, Y) \in \mathcal{F}(T) \bullet\left(\begin{array}{c}
t^{\prime} \text { cvg } t \wedge \\
Y \cap \text { inputs } \supseteq X \cap \text { inputs } \wedge \\
Y \cap \text { outputs } \subseteq X \cap \text { out puts }
\end{array}\right) \\
& \equiv\left[t^{\prime} \operatorname{cvg} t,\right. \text { Lemma 4.1] } \\
& \forall\left(t^{\prime}, X\right) \in \mathcal{F}\left(T^{\prime}\right), \exists(t, Y) \in \mathcal{F}(T) \bullet\left(\begin{array}{c}
t^{\prime} \text { ecvg } t \wedge \\
Y \cap \text { inputs } \supseteq X \cap \text { inputs } \wedge \\
Y \cap \text { outputs } \subseteq X \cap \text { out puts }
\end{array}\right) \\
& \Rightarrow[\mathrm{I} / \mathrm{O} \text { extended convergent behaviour (Definition } 4.4)] \\
& T^{\prime} \text { io_ecvg } T
\end{aligned}
$$

In Section 2.2 we we have motivated the need for behavioural convergence through a simple specification of a TV remote control extension; we took that opportunity to compare convergence with other relations such as ioco (input-output conformance) (BIJL; RENSINK; TRETMANS, 2004) and behavioural subtyping as proposed by (WEHRHEIM, 2003).

To conclude that discussion we need to consider another alternative to introduce new events when extending behavioural specifications: stuttering (LAMPORT, 1994; BOITEN, 2014). In Definitions 4.2 and 4.4, a new-in-context input marks the start of two possible kinds of extensions: the introduction of new functionalities (recall the adjustment of brightness and contrast in the TV remove control example in Section 2.2) and, the realisation (implementation) of existing functionalities (for example, the implementation of the withdraw operation in the bank system we present in Section 5.1). Otherwise, as pointed by (LAMPORT, 1994), stuttering is a mechanism to refine the grain of atomicity of abstract events, explaining them by a sequence of finer grain events. Therefore, the stuttering focus is on implementation, while convergence goes beyond and allows the introduction of new functionalities, which may also use existing events with a different meaning (in distinct contexts).

To put this difference into a concrete shape, consider the clock process Clock ( $\mathrm{hh}, \mathrm{mm}$ ), where $\mathrm{hh}$ and $\mathrm{mm}$ stand by the current time in terms of hours and minutes, respectively. Clock updates hh and mm by communicating the events nextHH and nextMM, respectively. An implementation of Clock can be given by the process $\mathrm{Clock}^{\prime}(\mathrm{hh}, \mathrm{mm}, \mathrm{ss}$ ), which also registers the seconds on the state variable s s by communicating the event next SS. Stuttering relation identifies an uninterrupted sequence of sixty nextSS as an implementation of the event nextMM. Therefore, Clock' does not extend the capabilities of Clock, but realize nextMM by a sequence of stuttering steps (nextSS events). On the other hand, the relation 
between TV_RC' and TV_RC (Section 2.2) cannot be captured by stuttering because the former introduces new functionalities in regard of the latter. Both relations (between Clock and Clock' and between TV_RC and TV_RC' ) can be captured by convergence, what makes it more general than stuttering, and still capable of differentiating inputs from outputs and to guarantee substitutability as we prove on the rest of this chapter.

Now that we have motivated, defined and exemplified convergence, we define inheritance relations for $\mathcal{B R J C}$ based on the notion of convergence.

\section{BRIC inheritance}

Our definition of inheritance deals with component structural and behavioural aspects (Definition 4.7). Structurally, it guarantees that the inherited component preserves at least its parent's channels and their types $\left(\mathcal{R}_{T} \subseteq \mathcal{R}_{T^{\prime}}\right)$. Regarding behaviour, they are related by convergence. Additionally, it guarantees, for substitutability purposes, that the inherited component must refine the protocols exhibited by common channels (default channel congruence, as in Definition 4.5) or that additional inputs (new in context, see Definitions 4.24.4) over common channels are not exercised by any possible client of its parent (input channel congruence, Definition 4.6).

Definition 4.5 (Default channel congruence). An I/O process $T_{d c}$ has a default congruent channel to another $I / O$ process $T$, lets say $c\left(T_{d c} \mathbf{d e f}_{-\operatorname{cong}_{\{c\}}} T\right)$, when there is a failures refinement relation between their projections over $c$, formally:

$$
\mathcal{F}\left(T_{d c} \uparrow c\right) \subseteq \mathcal{F}(T \uparrow c)
$$

Definition 4.6 (Input channel congruence). An I/O process $T_{i c}$ has an input congruent channel to an $I / O$ process $T$, say $c\left(T_{i c}\right.$ inp-cong $\left._{\{c\}} T\right)$, if after a common trace $t$, such that $(t, X) \in \mathcal{F}\left(T_{i c}\right)$ and $(t, Y) \in \mathcal{F}(T)$, the following holds: $T$ refuses to input (inputs $\subseteq Y$ ) and $T_{i c}$ refuses to input over $c(\{|c . i n|\} \subset X)$ or $T$ refuses to input over $c(\{|c . i n|\} \subseteq Y)$ and $T_{i c}$ refuses the events on $c$ that can be communicated by $T\left(\{|c . i n|\} \backslash\left(\alpha T_{i c} \backslash \alpha T\right) \subset X\right)$. Formally we have:

$$
\forall(t, X) \in \mathcal{F}\left(T_{i c}\right) \bullet \exists(t, Y) \in \mathcal{F}(T) \Rightarrow\left(\begin{array}{c}
\text { inputs } \subseteq Y \Rightarrow\{|c . i n|\} \subset X \\
\vee \\
\{\mid c . \text { in } \mid\} \subseteq Y \Rightarrow\{|c . i n|\} \backslash\left(\alpha T_{i c} \backslash \alpha T\right) \subset X
\end{array}\right)
$$

\section{provided}

$(t, X)$ and $(t, Y)$ are maximal failures

$$
\equiv\left(\exists\left(t, X^{\prime}\right) \in \mathcal{F}\left(T_{i c}\right) \Rightarrow X^{\prime} \subseteq X\right) \wedge\left(\exists\left(t, Y^{\prime}\right) \in \mathcal{F}(T) \Rightarrow Y^{\prime} \subseteq Y\right)
$$

By Definition 2.4, I/O processes offer inputs in external choice and outputs in internal 
choice. It restricts the specification of the clients of (I/O processes that can be connected with) these processes. Channel congruence aims at paving a safe way to extend a specification by using convergence, but without making it incompatible with (generating deadlock when in parallel with) its clients; it does not reduce possible inputs, but disciplines the way in which existing inputs can be used in convergent extensions. The most simple, but restrictive, form of achieving this is by guaranteeing that the protocol over a channel must be refined, in the failures classical sense (Definition 4.5). A more flexible way is given by Definition 4.6: I/O processes $T_{i c}$ and $T$ are input congruent on an I/O channel $c$ if, after both have done the same trace $t$, either of the following is true:

(a) if $T$ cannot input, then $T_{i c}$ cannot input on $c$ : it avoids $T$ 's clients of deadlocking when interacting with $T_{i c}$, since these clients do not communicate (after the trace $t$ ) outputs on $c$ to $T$, otherwise they deadlock; therefore $T_{i c}$, as $T$, cannot expect inputs on $c$ after the trace $t$;

(b) if $T$ is not able to input on $c, T_{i c}$ can only do it for events outside $T$ 's alphabet: it avoids $T$ 's clients to engage in a possible unexpected communication over $c$, that can (but not necessarily) lead to deadlock. It is worth saying that in places where $T$ can input over $c, T_{i c}$ can also input new-in-context events over $c$; it is possible because they are offered in external choice, so $T$ 's clients will not be able to communicate these new-in-context inputs offered by $T_{i c}$. This happens because $T$ 's clients are not ready to engage in these new-in-context inputs, otherwise, this would mean that their composition with $T$ deadlock, because $T$ is not able to offer such new-in-context inputs.

The intent of the provided clause in the following definition is to guarantee that, when interacting with $T^{\prime}$, a component originally designed to interact with $T$ will not engage in $T^{\prime}$ extensions trigged by inputs also used by $T$, which in $T^{\prime}$ have different meaning. For example, recall the behaviour of TV_RC' (Section 2.2), the input events up and down, which appear after sett, have a different meaning of those offered initially by TV_RC' (and also by TV_RC), but these events are preceded bysett followed by brig or cont events; since Granpa (which only knows TV_RC) cannot communicate sett, he can never be trapped by the new usages for up and down events.

Definition 4.7 (BRIC inheritance). Consider $T$ and $T^{\prime}$ two $\mathcal{B} \mathcal{R J C}$ components, such that $\mathcal{R}_{T} \subseteq$ $\mathcal{R}_{T^{\prime}}$. We say that $T^{\prime}$ inherits from $T$ :

- by convergence: $T \leftarrow_{{ }_{\text {cvg }}} T^{\prime} \Leftrightarrow \mathcal{B}_{T^{\prime}}$ io_cvg $\mathcal{B}_{T}$

- by extended convergence: $T \leftarrow_{\text {ecvg }} T^{\prime} \Leftrightarrow \mathcal{B}_{T^{\prime}}$ io_ecvg $\mathcal{B}_{T}$

provided

$\forall c: \mathcal{C}_{T} \bullet\left(\mathcal{B}_{T^{\prime}}\right.$ def-cong $\left.\{c\} \mathcal{B}_{T}\right) \vee\left(\mathcal{B}_{T^{\prime}}\right.$ inp-cong $\left._{\{c\}} \mathcal{B}_{T}\right)$ 
Theorem 4.1 states that the refinement and inheritance relations form a hierarchy (Figure 4.4). Component refinement is the strongest relation between $\mathcal{B R J C}$ components; moreover, it implies inheritance.Therefore we can see refinement as the strongest form of inheritance. As expected, inheritance by convergence is a more strict form of inheritance than extended convergence, as their names suggest.

Theorem 4.1 (Hierarchy). The relations $\sqsubseteq_{\mathcal{B}}, \leftarrow_{c v g}$ and $\leftarrow_{e c v g}$ form a hierarchy: $\sqsubseteq_{\mathcal{B}} \subseteq{ }_{c v g} \subseteq \triangleleft_{e c v g}$. Assume in the following proof $T$ and $T^{\prime}$ are components.

Proof.

\section{[First part]}

$\left[T \sqsubseteq_{\mathcal{B}} T^{\prime}\right.$ (Definition 3.1)]

$\mathcal{B}_{T} \sqsubseteq_{\mathrm{F}} \mathcal{B}_{T^{\prime}} \wedge \mathcal{C}_{T}=\mathcal{C}_{T^{\prime}} \wedge \forall c: \mathcal{C}_{T} \bullet \mathcal{R}_{T}(c) \subseteq \mathcal{R}_{T^{\prime}}(c)$

$\Rightarrow$ [failures semantics $\wedge$ set theory $\wedge$ hiding semantics]

$\mathcal{B}_{T} \sqsubseteq_{\mathrm{F}} \mathcal{B}_{T^{\prime}} \wedge \mathcal{C}_{T}=\mathcal{C}_{T^{\prime}} \wedge$

$\mathcal{R}_{T} \subseteq \mathcal{R}_{T^{\prime}} \wedge \forall c: \mathcal{C}_{T} \bullet \mathcal{B}_{T} \uparrow c \sqsubseteq_{\mathrm{F}} \mathcal{B}_{T^{\prime}} \uparrow c$

$\Rightarrow$ [default channel congruence (Definition 4.5)]

$\mathcal{B}_{T} \sqsubseteq \mathrm{F} \mathcal{B}_{T^{\prime}} \wedge \mathcal{C}_{T}=\mathcal{C}_{T^{\prime}} \wedge$

$\forall c: \mathcal{C}_{T} \bullet \mathcal{B}_{T^{\prime}}$ def-cong $\{c\} \mathcal{B}_{T}$

$\Rightarrow[\mathrm{I} / \mathrm{O}$ convergent behaviour (Definition 4.2) $\wedge$ failures semantics $]$

$\mathcal{B}_{T^{\prime}}$ io_cvg $\mathcal{B}_{T} \wedge \mathcal{C}_{T}=\mathcal{C}_{T^{\prime}} \wedge$

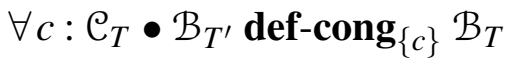

$\Rightarrow[\mathcal{B R J \mathcal { C }}$ inheritance (Definitions 4.7)]

$T \leftarrow{ }_{c v g} T^{\prime}$

\section{[Second part]}

$\left[T \leftarrow_{c v g} T^{\prime}\right.$ (Definition 4.7)]

$\mathcal{B}_{T^{\prime}}$ io_cvg $\mathcal{B}_{T} \wedge \mathcal{R}_{T} \subseteq \mathcal{R}_{T^{\prime}} \wedge \forall c: \mathcal{C}_{T} \bullet\left(\mathcal{B}_{T^{\prime}}\right.$ def-cong $\left.\{c\} \mathcal{B}_{T}\right) \vee\left(\mathcal{B}_{T^{\prime}}\right.$ inp-cong $\left._{\{c\}} \mathcal{B}_{T}\right)$

$\Rightarrow[$ io_cvg $\subseteq$ io_ecvg (Lemma 4.2)]

$\mathcal{B}_{T^{\prime}}$ io_ecvg $\mathcal{B}_{T} \wedge \mathcal{R}_{T} \subseteq \mathcal{R}_{T^{\prime}} \wedge \forall c: \mathcal{C}_{T} \bullet\left(\mathcal{B}_{T^{\prime}}\right.$ def-cong $\left.\{c\} \mathcal{B}_{T}\right) \vee\left(\mathcal{B}_{T^{\prime}}\right.$ inp-cong $\left.\{c\} \mathcal{B}_{T}\right)$

$\Rightarrow[\mathcal{B R J \mathcal { C }}$ inheritance (Definition 4.7)]

$T \leftarrow_{e c v g} T^{\prime}$ 
Figure 4.4: Hierarchy of $\mathcal{B R J C}$ relations

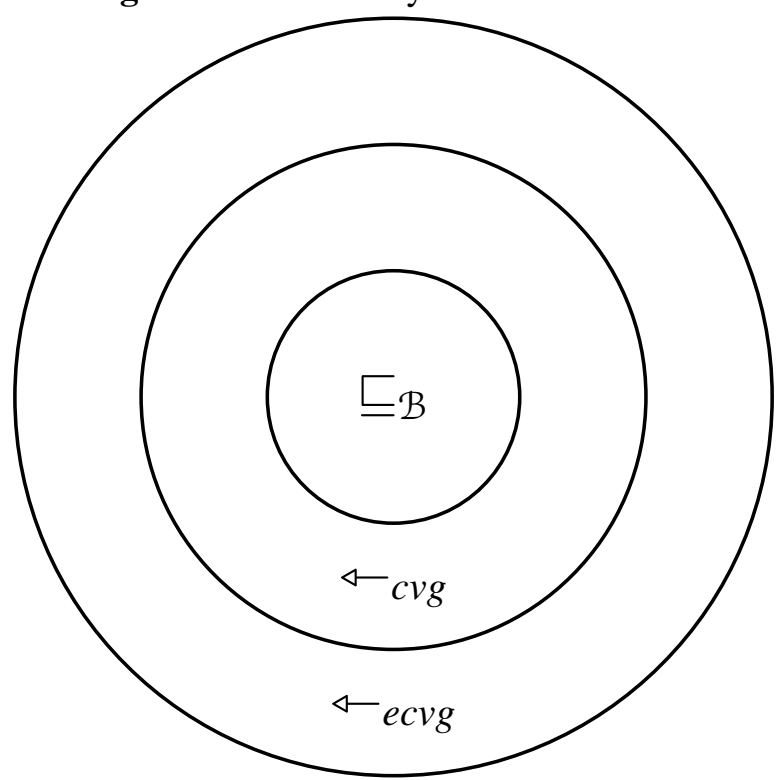

Source: José Dihego

The next lemma allows us to relate refinement (Definition 3.1) and inheritance (Definition 4.7), see Figure 4.5. Suppose one has specified a component $T$ that meets the initial requirements of a specification; we have two ways to evolve $T$ : (1) refining its abstract behaviour into a more concrete one (by the component $T_{c o n}$ ), and then extracting, by inheritance, reusable components from it (lets say $T_{a b s}$ ), or (2) by extracting, trough inheritance, at first, an abstraction from $T$ (lets say $U$ ) and then refining such an abstraction as the component $T_{a b s}$. This lemma deals with the first case: if we have some component $T$, which is refined by $T_{c o n}$, from where we identify, by inheritance, an abstraction $T_{a b s}$, we can guarantee that there is some component $U$ that is refined by $T_{a b s}$ and is extended by $T$ (the second case is analogous).

We highlight that, in the general case, inheritance does not preserve refinement nor the other way round. Again consider the components $T, U, T_{c o n}$ and $T_{a b s}$ in Figure 4.5, such that $U \sqsubseteq_{\mathcal{B}} T_{a b s}, U \triangleleft_{e c v g} T$ and $T_{a b s} \triangleleft_{e c v g} T_{c o n}$ : it is not always true that $T \sqsubseteq_{\mathcal{B}} T_{\text {con }}$. Similarly, it is not the case that refinement preserves inheritance in general. Therefore, given the limitations of our relations, Lemma 4.3 presents a way to interchange refinement and inheritance to evolve component specifications in BRJE (Figure 4.5).

Lemma 4.3 (Inheritance and Refinement). Let $T, T_{c o n}$ and $T_{a b s}$ be component contracts, such that $T \sqsubseteq_{\mathcal{B}} T_{\text {con }}$ and $T_{a b s} \leftarrow_{e c v g} T_{\text {con }}$, then there must be some component $U$, such that $U \sqsubseteq_{\mathcal{B}} T_{a b s}$ and $U \leftarrow_{e c v g} T$.

Proof. This proof consists in defining $U$ based on $T, T_{c o n}$ and $T_{a b s}$ and their relations $T \sqsubseteq \mathcal{B} T_{c o n}$ and $T_{a b s} \leftarrow_{e c v g} T_{c o n}$.

$\left[\begin{array}{l}T \sqsubseteq_{\mathcal{B}} T_{\text {con }} \text { (Definition 3.1) } \wedge T_{a b s} \triangleleft{ }_{e c v g} T_{\text {con }} \text { (Definition 4.7) } \\ \wedge \mathcal{B}_{T_{a b s}} \text { strong output decisiveness (Definition 2.4) }\end{array}\right]$ 
$\mathcal{C}_{U}=\mathcal{C}_{T_{a b s}} \wedge \mathcal{R}_{U}=\mathcal{R}_{T_{a b s}} \wedge \forall s: \mathcal{T}\left(\mathcal{B}_{T_{a b s}}\right) \bullet$ out $\left(\mathcal{B}_{T_{a b s}}, s\right) \subseteq$ out $\left(\mathcal{B}_{U}, s\right)$

$\Rightarrow\left[\mathcal{B}_{T_{a b s}}\right.$ input determinism (Definition 2.4)]

$\mathcal{C}_{U}=\mathcal{C}_{T_{a b s}} \wedge \mathcal{R}_{U}=\mathcal{R}_{T_{a b s}} \wedge \forall s: \mathcal{T}\left(\mathcal{B}_{T_{a b s}}\right) \bullet$ out $\left(\mathcal{B}_{T_{a b s}}, s\right) \subseteq$ out $\left(\mathcal{B}_{U}, s\right) \wedge \operatorname{in}\left(\mathcal{B}_{T_{a b s}}, s\right)=\operatorname{in}\left(\mathcal{B}_{U}, s\right)$

$\Rightarrow\left[\mathcal{B}_{U}\right.$ is an I/O process (Definition 2.4) $\wedge \mathcal{B R J \mathcal { C }}$ refinement (Definition 3.1)]

$U \sqsubseteq_{\mathcal{B}} T_{a b s}\left(T_{a b s}\right.$ can be , potentially, more deterministic than $U$ )

$\Rightarrow\left[T \sqsubseteq_{\mathcal{B}} T_{\text {con }} \wedge T_{\text {abs }} \leftarrow_{\text {ecvg }} T_{\text {con }}\right]$

$U \sqsubseteq_{\mathcal{B}} T_{a b s} \wedge \forall(t, X): \mathcal{F}\left(\mathcal{B}_{T_{c o n}}\right) \bullet(t, X) \in \mathcal{F}\left(\mathcal{B}_{T}\right) \wedge \forall(t, X): \mathcal{F}\left(\mathcal{B}_{T_{a b s}}\right) \bullet(t, X) \in \mathcal{F}\left(\mathcal{B}_{U}\right) \wedge$

$\left.\forall(t, X) \in \mathcal{F}\left(\mathcal{B}_{T_{\text {con }}}\right), \exists(s, Y) \in \mathcal{F}\left(\mathcal{B}_{T_{\text {abs }}}\right) \bullet t \operatorname{ecvg} s \wedge\left(\begin{array}{c}Y \cap \text { inputs } \supseteq X \cap \text { inputs } \wedge \\ Y \cap \text { outputs } \subseteq X \cap \text { outputs }\end{array}\right)\right)$

$\Rightarrow\left[\begin{array}{l}\text { I/O process strong output decisiveness and input determinism (Definition 2.4), } \\ \text { default channel congruence (Definition 4.5), input channel congruence (Definition 4.6) }\end{array}\right]$

$U \sqsubseteq_{\mathcal{B}} T_{a b s} \wedge \forall(t, X): \mathcal{F}\left(\mathcal{B}_{T_{c o n}}\right) \bullet(t, X) \in \mathcal{F}\left(\mathcal{B}_{T}\right) \wedge \forall(t, X): \mathcal{F}\left(\mathcal{B}_{T_{a b s}}\right) \bullet(t, X) \in \mathcal{F}\left(\mathcal{B}_{U}\right) \wedge$

$\forall(t, X) \in \mathcal{F}\left(\mathcal{B}_{T_{\text {con }}}\right), \exists(s, Y) \in \mathcal{F}\left(\mathcal{B}_{T_{\text {abs }}}\right) \bullet t$ ecvg $s \wedge\left(\begin{array}{c}Y \cap \text { inputs } \supseteq X \cap \text { inputs } \wedge \\ Y \cap \text { outputs } \subseteq X \cap \text { out puts }\end{array}\right) \wedge$

$\mathcal{C}_{T}=\mathcal{C}_{T_{\text {con }}} \wedge \mathcal{R}_{T}=\mathcal{R}_{T_{\text {con }}} \wedge \forall s: \mathcal{T}\left(\mathcal{B}_{T_{\text {con }}}\right) \bullet \operatorname{out}\left(\mathcal{B}_{T_{\text {con }}}, s\right) \subseteq \operatorname{out}\left(\mathcal{B}_{T}, s\right) \wedge \operatorname{in}\left(\mathcal{B}_{T_{\text {con }}}, s\right)=\operatorname{in}\left(\mathcal{B}_{T}, s\right) \wedge$

$\forall c: \mathcal{C}_{T_{a b s}} \bullet\left(\mathcal{B}_{T_{c o n}}\right.$ def-cong $\left.\{c\} \mathcal{B}_{T_{a b s}}\right) \vee\left(\mathcal{B}_{T_{c o n}}\right.$ inp-cong $\left.\{c\} \mathcal{B}_{T_{a b s}}\right)$

$\Rightarrow$ set theory $\wedge \sqsubseteq_{\mathrm{F}}$ transitivity $]$

$\forall(t, X) \in \mathcal{F}\left(\mathcal{B}_{T}\right), \exists(s, Y) \in \mathcal{F}\left(\mathcal{B}_{U}\right) \bullet t \operatorname{ecvg} s \wedge\left(\begin{array}{c}Y \cap \text { inputs } \supseteq X \cap \text { inputs } \wedge \\ Y \cap \text { outputs } \subseteq X \cap \text { outputs }\end{array}\right) \wedge$

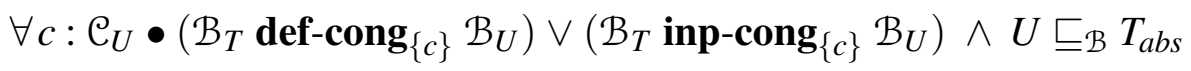

$\Rightarrow[\mathcal{B R J \mathcal { C }}$ inheritance (Definition 4.7)]

$\Rightarrow U \sqsubseteq_{\mathcal{B}} T_{a b s} \wedge U \leftarrow_{e c v g} T$

We prove, in Lemma 4.4, that $\mathcal{B R J \mathcal { C }}$ inheritance preserves deadlock freedom. A more interesting result, proved in Theorem 4.2, guarantees that a component $T^{\prime}$ can replace $T$, in any context produced by the $\mathcal{B} \mathcal{R J} \mathcal{C}$ composition rules, without introducing deadlock, provided $T^{\prime}$ inherits by convergence from $T$.

Lemma 4.4 (BRJe inheritance preserves deadlock freedom). Consider $T$ and $T^{\prime}$ two $\mathcal{B R J C}$ components, such that $T$ is deadlock free. If $T \leftarrow_{e c v g} T^{\prime}$ then $T^{\prime}$ is deadlock free. 
Figure 4.5: $\mathcal{B R J C}$ inheritance and refinement

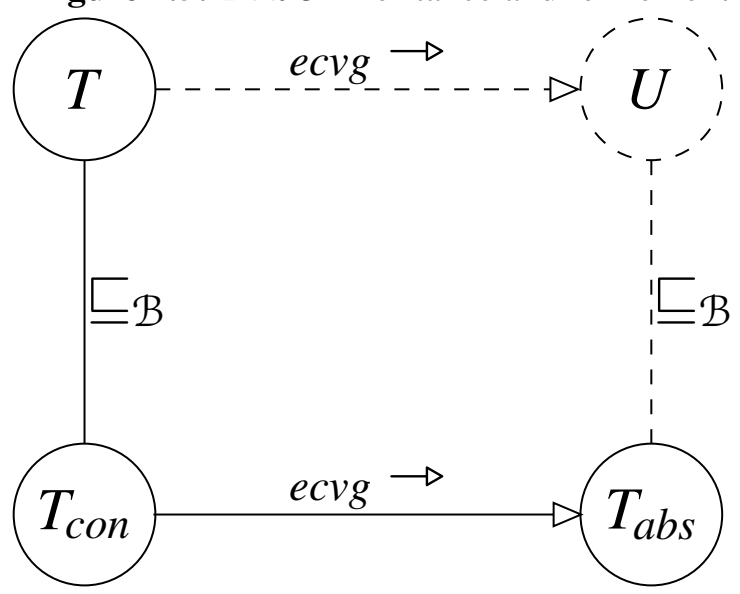

Source: José Dihego

Proof.

$\left[T \leftarrow_{e c v g} T^{\prime}\right.$ (Definition 4.7) $\wedge T$ is deadlock free $]$

$\mathcal{B}_{T^{\prime}}$ io_ecvg $\mathcal{B}_{T} \wedge \forall s \in \Sigma^{*} \bullet\left(s, \Sigma^{\checkmark}\right) \notin \operatorname{failures}\left(\mathcal{B}_{T}\right)$

$\Rightarrow[\mathrm{I} / \mathrm{O}$ extended convergent behaviour (Definition 4.4)]

$\forall\left(t^{\prime}, X\right) \in \mathcal{F}\left(\mathcal{B}_{T^{\prime}}\right), \exists(t, Y) \in \mathcal{F}\left(\mathcal{B}_{T}\right) \bullet t^{\prime} \operatorname{ecvg} t \wedge\left(\begin{array}{c}Y \cap \text { inputs } \supseteq X \cap \text { inputs } \wedge \\ Y \cap \text { outputs } \subseteq X \cap \text { outputs }\end{array}\right)$

$\wedge \forall s \in \Sigma^{*} \bullet\left(s, \Sigma^{\checkmark}\right) \notin$ failures $\left(\mathcal{B}_{T}\right)$

$\Rightarrow[$ set theory $]$

$\forall s \in \Sigma^{*} \bullet\left(s, \Sigma^{\checkmark}\right) \notin$ failures $\left(\mathcal{B}_{T^{\prime}}\right)$

$\Rightarrow$ [deadlock freedom (Section 2.1.2)]

$T^{\prime}$ is deadlock free

Theorem 4.2 (Substitutability). Let $T$, $T^{\prime}$ be two components such that $T \leftarrow_{\text {ecvg }} T^{\prime}$. Consider $S[T]$ a deadlock free component contract, where $T$ is a deadlock free component contract that appears within the context $S$, then $S\left[T^{\prime}\right]$ is deadlock free.

Proof. The proof follows by structural induction on the composition operators of $\mathcal{B R J C}$ (the trivial case where $S[T]=T$ is proved by Lemma 4.4). Assuming it holds for $T$, we prove that it holds for the following cases: $T$ [III] $Q, T[c \leftrightarrow z] Q, T[c \hookrightarrow z]$ and $T[c \hookrightarrow z]$, where $Q$ is a BRJC component. As $S[T]$ and $Q$ are deadlock free we know that when communicating with $T, Q$ never generates an output that cannot be consumed by the respective input of $T$, and vice versa, after both have done a common trace (common by appropriate bidirectional mapping functions, recall from Section 2.3.3). This prevents the emergence of ungranted requests, a common source of deadlock as described by (ROSCOE, 1998); the same reasoning applies when $T$ is self 
connected by two of its channels. We also assume $Q$ is capable of stimulating all expected inputs in any point of $T$, so after a common trace, $Q$ can output anything $P$ is able to handshake by its inputs. This is particularly important in our proof because it constitutes the worst scenario for substitutability: when $Q$, acting as a client, is able to exercise (when given the grant, by $P$ offering inputs in external choice) the largest possible number of execution flows of $P$. As $T \leftarrow_{e c v g} T^{\prime}$, we have that $\mathcal{C}_{T} \subseteq \mathcal{C}_{T^{\prime}}$, and to compose $T^{\prime}$ with $Q$ we require $\mathcal{C}_{T^{\prime}} \backslash \mathcal{C}_{T}$ and $\mathcal{C}_{Q}$ to be disjoint.

[First case $T[|| \mid] Q]$

$\left[\begin{array}{l}T \leftarrow_{\text {ecvg }} T^{\prime} \wedge\left(\left(\mathcal{C}_{T^{\prime}} \backslash \mathcal{C}_{T}\right) \cap \mathcal{C}_{Q}=\emptyset\right) \wedge \\ \text { inheritance preserves deadlock freedom (Lemma 4.4) }\end{array}\right]$

$\mathcal{C}_{T^{\prime}} \cap \mathcal{C}_{Q}=\emptyset \wedge T^{\prime}$ is a deadlock free contract

$\Rightarrow\left[\begin{array}{l}Q \text { is a deadlock free contract } \wedge \text { interleave composition (Definition 2.16) } \wedge \\ \text { deadlock free contract by construction (Theorem 2.1) }\end{array}\right]$

$T^{\prime}[|\||] Q$ is a deadlock free contract

[Second case $T[c \leftrightarrow z] Q]$

$\left[\begin{array}{l}T \leftarrow_{e c v g} T^{\prime} \wedge\left(\left(\mathcal{C}_{T^{\prime}} \backslash \mathcal{C}_{T}\right) \cap \mathcal{C}_{Q}=\emptyset\right) \wedge \text { communication composition (Definition 2.17) } \wedge \\ \text { inheritance preserves deadlock freedom (Lemma 4.4) }\end{array}\right]$

$\mathcal{C}_{T^{\prime}} \cap \mathcal{C}_{Q}=\emptyset \wedge T^{\prime}$ is a deadlock free contract

$\Rightarrow$ [asynchronous binary composition (Definition 2.14)]

$\mathcal{C}_{T^{\prime}} \cap \mathcal{C}_{Q}=\emptyset \wedge T^{\prime}$ is a deadlock free contract $\wedge$

$\mathcal{B}_{T[c \leftrightarrow z] Q}=\mathcal{B}_{T}\left\|_{\{|c|\}} B U F F_{I O}^{n}\left(R_{I O}^{c \rightarrow z}, R_{I O}^{z \rightarrow c}\right)\right\|_{\{|z|\}} \mathcal{B}_{Q}$

$\Rightarrow\left[\mathcal{B}_{T[c \leftrightarrow z] Q}\right.$ is deadlock free, hiding semantics $]$

$\mathcal{C}_{T^{\prime}} \cap \mathcal{C}_{Q}=\emptyset \wedge T^{\prime}$ is a deadlock free contract $\wedge$

$\forall t \in \mathcal{T}\left(\mathcal{B}_{T} \uparrow c\right) \bullet R_{I O}^{c \rightarrow z^{*}} t \in \mathcal{T}\left(\mathcal{B}_{Q} \uparrow z\right) \Rightarrow\left(\begin{array}{c}R_{I O}^{c \rightarrow z} \operatorname{out}\left(\mathcal{B}_{T} \uparrow c, t\right) \subseteq \operatorname{in}\left(\mathcal{B}_{Q} \uparrow z, R_{I O}^{c \rightarrow z^{*}} t\right) \\ \wedge \\ R_{I O}^{z \rightarrow c} \operatorname{out}\left(\mathcal{B}_{Q} \uparrow z, R_{I O}^{c \rightarrow z^{*}} t\right) \subseteq \operatorname{in}\left(\mathcal{B}_{T} \uparrow c, t\right)\end{array}\right)$

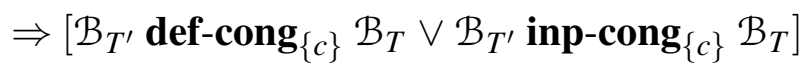

$\mathcal{C}_{T^{\prime}} \cap \mathcal{C}_{Q}=\emptyset \wedge T^{\prime}$ is a deadlock free contract $\wedge$

$\forall t: \Sigma^{*} \mid t \in \mathcal{T}(T \nmid c) \wedge t \in \mathcal{T}\left(T^{\prime} \uparrow c\right) \bullet$ out $\left(T^{\prime}, t\right) \subseteq$ out $(T, t) \wedge \operatorname{in}(T, t) \subseteq \operatorname{in}\left(T^{\prime}, t\right) \wedge$

$\mathcal{T}\left(\mathcal{B}_{T^{\prime}} \uparrow c\right) \cap \mathcal{T}\left(\mathcal{B}_{Q} \uparrow z\left[\left[R_{I O}^{z \rightarrow c}\right]\right]\right) \subseteq \mathcal{T}\left(\mathcal{B}_{T} \uparrow c\right) \cap \mathcal{T}\left(\mathcal{B}_{Q} \uparrow z\left[\left[R_{I O}^{z \rightarrow c}\right]\right]\right)$

$\Rightarrow$ [ communication composition (Definition 2.17)]

$T^{\prime}[c \leftrightarrow z] Q$ is a deadlock free contract 
[Third case $T[c \hookrightarrow z]]$

$\left[\begin{array}{c}T \leftarrow_{\text {ecvg }} T^{\prime} \wedge \text { feedback composition (Definition 2.18) } \\ \text { inheritance preserves deadlock freedom (Lemma 4.4) }\end{array}\right]$

$\mathrm{C}_{T} \subseteq \mathrm{C}_{T^{\prime}} \wedge T^{\prime}$ is a deadlock free contract

$\Rightarrow$ [asynchronous unary composition (Definition 2.15)]

$\mathrm{C}_{T} \subseteq \mathcal{C}_{T^{\prime}} \wedge T^{\prime}$ is a deadlock free contract $\wedge$

$\mathcal{B}_{T[c \hookrightarrow z]}=\mathcal{B}_{T}\|\|_{\{|c, z|\}} B U F F_{I O}^{n}\left(R_{I O}^{c \rightarrow z}, R_{I O}^{z \rightarrow c}\right)$

$\Rightarrow\left[\mathcal{B}_{T[c \hookrightarrow z]}\right.$ is deadlock free $\wedge \uparrow$ hiding semantics $]$

$\mathcal{C}_{T} \subseteq \mathcal{C}_{T^{\prime}} \wedge T^{\prime}$ is a deadlock free contract $\wedge$

$\forall t \in \mathcal{T}\left(\mathcal{B}_{T} \uparrow c\right) \bullet R_{I O}^{c \rightarrow z^{*}} t \in \mathcal{T}\left(\mathcal{B}_{T} \uparrow z\right) \Rightarrow\left(\begin{array}{c}R_{I O}^{c \rightarrow z} \operatorname{out}\left(\mathcal{B}_{T} \uparrow c, t\right) \subseteq \operatorname{in}\left(\mathcal{B}_{T} \uparrow z, R_{I O}^{c \rightarrow z^{*} t} t\right) \\ \wedge \\ R_{I O}^{z \rightarrow c} \operatorname{out}\left(\mathcal{B}_{T} \uparrow z, R_{I O}^{c \rightarrow z *} t\right) \subseteq \operatorname{in}\left(\mathcal{B}_{T} \uparrow c, t\right)\end{array}\right)$

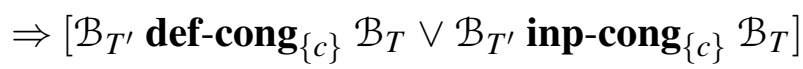

$\mathrm{C}_{T} \subseteq \mathrm{C}_{T^{\prime}} \wedge T^{\prime}$ is a deadlock free contract $\wedge$

$\forall t: \Sigma^{*} \mid t \in \mathcal{T}(T \uparrow c) \wedge t \in \mathcal{T}\left(T^{\prime} \uparrow c\right) \bullet \operatorname{out}\left(T^{\prime}, t\right) \subseteq$ out $(T, t) \wedge \operatorname{in}(T, t) \subseteq \operatorname{in}\left(T^{\prime}, t\right) \wedge$

$\mathcal{T}\left(\mathcal{B}_{T^{\prime}} \uparrow c\right) \cap \mathcal{T}\left(\mathcal{B}_{T} \uparrow z\left[\left[R_{I O}^{z \rightarrow c}\right]\right]\right) \subseteq \mathcal{T}\left(\mathcal{B}_{T} \uparrow c\right) \cap \mathcal{T}\left(\mathcal{B}_{T} \uparrow z\left[\left[R_{I O}^{z \rightarrow c}\right]\right]\right)$

$\Rightarrow$ [feedback composition (Definition 2.18)]

$T^{\prime}[c \hookrightarrow z]$ is a deadlock free contract

[ Fourth case $T\left[c^{\hookrightarrow} \rightarrow z\right]$ ] It is almost identical to the third part.

Note that this result also holds for the other two relations ( $\sqsubseteq_{\mathcal{B}}$ and $\leftarrow_{c v g}$ ), as a consequence of the hierarchy established in Theorem 4.1 .

Theorem 4.3 (Compositionality). Let $T$ and $T^{\prime}$ be two component contracts such that $T-$ ecvg $T^{\prime}$. Consider $S[T]$ a component contract, where $T$ appears within the context $S$, then $S[T] \triangleleft$ ecvg $S\left[T^{\prime}\right]$.

Proof. Taking the same structure of Theorem 4.2, this proof follows by structural induction on the composition operators of $\mathcal{B R J C}$. Assuming $T \triangleleft_{e c v g} T^{\prime}$, we consider four configurations for $S[T]: T[\|||] Q, T[c \leftrightarrow z] Q, T[c \hookrightarrow z]$ and $T\left[c_{\hookrightarrow}^{\hookrightarrow} z\right]$, where $Q$ is a $\mathcal{B R J C}$ component; we need to prove for each case that $S\left[T^{\prime}\right]$ inherits from $S[T]$. As $T \leftarrow_{e c v g} T^{\prime}$, we have that $\mathcal{C}_{T} \subseteq \mathcal{C}_{T^{\prime}}$, and to compose $T^{\prime}$ with $Q$ we require $\mathcal{C}_{T^{\prime}} \backslash \mathcal{C}_{T}$ and $\mathcal{C}_{Q}$ to be disjoint. 
$[$ First case $T[|| \mid] Q]$

$\left[\begin{array}{l}T \leftarrow_{\text {ecvg }} T^{\prime} \wedge\left(\mathcal{C}_{T^{\prime}} \cap \mathcal{C}_{Q}=\emptyset\right) \wedge T[\|\|] Q \text { is deadlock free, } \\ \text { substitutability of inheritance (Theorem 4.2) }\end{array}\right]$

$T^{\prime}[\|\|] Q$ is a deadlock free contract $\wedge$

$\forall\left(t^{\prime}, X\right) \in \mathcal{F}\left(\mathcal{B}_{T^{\prime}}\right), \exists(t, Y) \in \mathcal{F}\left(\mathcal{B}_{T}\right) \bullet t^{\prime} \operatorname{ecvg} t \wedge\left(\begin{array}{c}Y \cap \text { inputs } \supseteq X \cap \text { inputs } \wedge \\ Y \cap \text { outputs } \subseteq X \cap \text { outputs }\end{array}\right)$

$\Rightarrow$ [deadlock free process (ROSCOE, 1998)]

$\forall s \in \Sigma^{*} \bullet\left(s, \Sigma^{\checkmark}\right) \notin \mathcal{F}\left(\mathcal{B}_{T^{\prime}[\|\|] Q}\right) \wedge$

$\forall\left(t^{\prime}, X\right) \in \mathcal{F}\left(\mathcal{B}_{T^{\prime}}\right), \exists(t, Y) \in \mathcal{F}\left(\mathcal{B}_{T}\right) \bullet t^{\prime} \operatorname{ecvg} t \wedge\left(\begin{array}{c}Y \cap \text { inputs } \supseteq X \cap \text { inputs } \wedge \\ Y \cap \text { outputs } \subseteq X \cap \text { outputs }\end{array}\right)$

$\Rightarrow$ [interleave rule $[|l|]$ (Definition 2.16)]

$\forall s \in \Sigma^{*} \bullet\left(s, \Sigma^{\checkmark}\right) \notin \mathcal{F}\left(\mathcal{B}_{T^{\prime}[||]} Q\right) \wedge$

$\forall\left(t^{\prime}, X\right) \in \mathcal{F}\left(\mathcal{B}_{T^{\prime}}\right), \exists(t, Y) \in \mathcal{F}\left(\mathcal{B}_{T}\right) \bullet t^{\prime}$ ecvg $t \wedge\left(\begin{array}{c}Y \cap \text { inputs } \supseteq X \cap \text { inputs } \wedge \\ Y \cap \text { outputs } \subseteq X \cap \text { outputs } \\ \vee(\Sigma \backslash Y \subseteq X)\end{array}\right) \wedge$

$\mathcal{F}\left(\mathcal{B}_{T}|| \mathcal{B}_{Q}\right)=\bigcup\left\{\left(s_{0}|| \mid s_{1}, K \cup L\right) \mid K=L \wedge\left(s_{0}, K\right) \in \mathcal{F}\left(\mathcal{B}_{T}\right) \wedge\left(s_{1}, L\right) \in \mathcal{F}\left(\mathcal{B}_{Q}\right)\right\} \wedge$

$\mathcal{F}\left(\mathcal{B}_{T^{\prime}}|| \mathcal{B}_{Q}\right)=\bigcup\left\{\left(s_{0}|| \mid s_{1}, K \cup L\right) \mid K=L \wedge\left(s_{0}, K\right) \in \mathcal{F}\left(\mathcal{B}_{T^{\prime}}\right) \wedge\left(s_{1}, L\right) \in \mathcal{F}\left(\mathcal{B}_{Q}\right)\right\}$

$\Rightarrow$ [set theory, failures semantics]

$\forall\left(t^{\prime}, X\right) \in \mathcal{F}\left(\mathcal{B}_{T^{\prime}} \| \mid \mathcal{B}_{Q}\right), \exists(t, Y) \in \mathcal{F}\left(\mathcal{B}_{T} \| \mathcal{B}_{Q}\right) \bullet t^{\prime} \operatorname{ecvg} t \wedge\left(\begin{array}{c}Y \cap \text { inputs } \supseteq X \cap \text { inputs } \wedge \\ Y \cap \text { outputs } \subseteq X \cap \text { out puts }\end{array}\right)$

$\Rightarrow\left[\begin{array}{l}\text { default channel congruence (Definition 4.5), input channel congruence (Definition 4.6), } \\ \left(\mathcal{C}_{T^{\prime}} \backslash \mathcal{C}_{T}\right) \cap \mathcal{C}_{Q}=\emptyset, \mathrm{I} / \mathrm{O} \text { extended convergent behaviour (Definition 4.4) }\end{array}\right]$

$\mathcal{B}_{T^{\prime}}\left\|\mathcal{B}_{Q} \operatorname{ecvg} \mathcal{B}_{T}\right\| \mathcal{B}_{Q} \wedge$

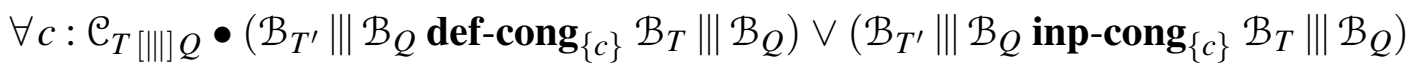

$\Rightarrow[\mathcal{B R J \mathcal { C }}$ inheritance (Definition 4.7)]

$T[|| \mid] Q \leftarrow_{e c v g} T^{\prime}[|| \mid] Q$

[Second case $T[c \leftrightarrow z] Q]$

$\left[\begin{array}{l}T \leftarrow_{e c v g} T^{\prime} \wedge\left(\mathcal{C}_{T^{\prime}} \cap \mathcal{C}_{Q}=\emptyset\right) \wedge T[c \leftrightarrow z] Q \text { is deadlock free, } \\ \text { substitutability of inheritance (Theorem 4.2) }\end{array}\right]$ 
$T^{\prime}[c \leftrightarrow z] Q$ is a deadlock free contract $\wedge$

$\forall\left(t^{\prime}, X\right) \in \mathcal{F}\left(\mathcal{B}_{T^{\prime}}\right), \exists(t, Y) \in \mathcal{F}\left(\mathcal{B}_{T}\right) \bullet t^{\prime} \operatorname{ecvg} t \wedge\left(\begin{array}{c}Y \cap \text { inputs } \supseteq X \cap \text { inputs } \wedge \\ Y \cap \text { outputs } \subseteq X \cap \text { outputs }\end{array}\right)$

$\Rightarrow$ [deadlock free process (ROSCOE, 1998)]

$\forall s \in \Sigma^{*} \bullet\left(s, \Sigma^{\checkmark}\right) \notin \mathcal{F}\left(\mathcal{B}_{T^{\prime}[c \leftrightarrow z] Q}\right) \wedge$

$\forall\left(t^{\prime}, X\right) \in \mathcal{F}\left(\mathcal{B}_{T^{\prime}}\right), \exists(t, Y) \in \mathcal{F}\left(\mathcal{B}_{T}\right) \bullet t^{\prime} \operatorname{ecvg} t \wedge\left(\begin{array}{c}Y \cap \text { inputs } \supseteq X \cap \text { inputs } \wedge \\ Y \cap \text { outputs } \subseteq X \cap \text { outputs }\end{array}\right)$

$\Rightarrow$ [communication rule (Definition 2.17)]

$\forall s \in \Sigma^{*} \bullet\left(s, \Sigma^{\checkmark}\right) \notin \mathcal{F}\left(\mathcal{B}_{T^{\prime}[c \leftrightarrow z] Q}\right) \wedge \mathcal{B}_{T[c \leftrightarrow z] Q}=\mathcal{B}_{T}\left\|\underset{\{|c|\}}{ } B U F F_{I O}^{n}\left(R_{I O}^{c \rightarrow z}, R_{I O}^{z \rightarrow c}\right)\right\|_{\{|z|\}} \mathcal{B}_{Q}$

$\forall\left(t^{\prime}, X\right) \in \mathcal{F}\left(\mathcal{B}_{T^{\prime}}\right), \exists(t, Y) \in \mathcal{F}\left(\mathcal{B}_{T}\right) \bullet t^{\prime} \operatorname{ecvg} t \wedge\left(\begin{array}{c}Y \cap \text { inputs } \supseteq X \cap \text { inputs } \wedge \\ Y \cap \text { outputs } \subseteq X \cap \text { outputs }\end{array}\right)$

$\Rightarrow\left[B U F F_{I O}^{n}\left(R_{I O}^{c \rightarrow z}, R_{I O}^{z \rightarrow c}\right)\right.$ does not reorder or lose events (Definition 2.14) (ROSCOE, 2005)] $\forall s \in \Sigma^{*} \bullet\left(s, \Sigma^{\checkmark}\right) \notin \mathcal{F}\left(\mathcal{B}_{T^{\prime}[c \leftrightarrow z] Q}\right) \wedge \mathcal{B}_{T[c \leftrightarrow z] Q}=\mathcal{B}_{T}\left\|B U F F_{I O}^{n}\left(R_{I O}^{c \rightarrow z}, R_{I O}^{z \rightarrow c}\right)\right\| \|_{\{|z|\}} \mathcal{B}_{Q} \wedge$ $\forall\left(t^{\prime}, X\right) \in \mathcal{F}\left(\mathcal{B}_{T^{\prime}}\right), \exists(t, Y) \in \mathcal{F}\left(\mathcal{B}_{T}\right) \bullet t^{\prime} \operatorname{ecvg} t \wedge\left(\begin{array}{c}Y \cap \text { inputs } \supseteq X \cap \text { inputs } \wedge \\ Y \cap \text { outputs } \subseteq X \cap \text { outputs }\end{array}\right) \wedge$

$\mathcal{F}\left(\mathcal{B}_{T[c \leftrightarrow z] Q}\right)=\left\{\left(s_{0} \|_{f} s_{1}, K \cup L\right) \mid K \backslash\{|f|\}=L \backslash\{|f|\} \wedge\left(s_{0}, K\right) \in \mathcal{F}\left(\mathcal{B}_{T}[[\right.\right.$ c.in/f.out, c.out $/ f$.in $\left.]]\right)$

$\left(s_{1}, L\right) \in \mathcal{F}\left(\mathcal{B}_{Q}[[z . i n / f\right.$.in,z.out $/$ f.out $\left.\left.]]\right)\right\}[[$ f.out $/$ c.in,$f$.in $/$ c.out,$f / z]] \wedge$

$\mathcal{F}\left(\mathcal{B}_{T^{\prime}[c \leftrightarrow z] Q}\right)=\left\{\left(s_{0} \|_{f} s_{1}, K \cup L\right) \mid K \backslash\{|f|\}=L \backslash\{|f|\} \wedge\left(s_{0}, K\right) \in \mathcal{F}\left(\mathcal{B}_{T^{\prime}}[[c\right.\right.$. in $/$ f.out, c.out $/ f$. in $\left.]]\right)$

$\left(s_{1}, L\right) \in \mathcal{F}\left(\mathcal{B}_{Q}[[z\right.$. in $/ f$.in,z.out $/ f$.out $\left.\left.]]\right)\right\}[[f$.out $/$ c.in,$f$.in $/$ c.out,$f / z]]$

$\Rightarrow$ [set theory, failures semantics, rename]

$\forall\left(t^{\prime}, X\right) \in \mathcal{F}\left(\mathcal{B}_{T^{\prime}[c \leftrightarrow z] Q}\right), \exists(t, Y) \in \mathcal{F}\left(\mathcal{B}_{T[c \leftrightarrow z] Q}\right) \bullet t^{\prime} \operatorname{ecvg} t \wedge\left(\begin{array}{c}Y \cap \text { inputs } \supseteq X \cap \text { inputs } \wedge \\ Y \cap \text { outputs } \subseteq X \cap \text { out puts }\end{array}\right)$

$\Rightarrow\left[\begin{array}{l}\text { default channel congruence (Definition 4.5), input channel congruence (Definition 4.6), } \\ \left(\mathcal{C}_{T^{\prime}} \backslash \mathcal{C}_{T}\right) \cap \mathcal{C}_{Q}=\emptyset, \mathrm{I} / \mathrm{O} \text { extended convergent behaviour (Definition 4.4) }\end{array}\right]$

$\mathcal{B}_{T^{\prime}[c \leftrightarrow z] Q} \operatorname{ecvg} \mathcal{B}_{T[c \leftrightarrow z] Q} \wedge$

$\forall c: \mathcal{C}_{T[c \leftrightarrow z]} \bullet\left(\mathcal{B}_{T^{\prime}[c \leftrightarrow z] Q}\right.$ def-cong $\left.\{c\} \mathcal{B}_{T[c \leftrightarrow z]}\right) \vee\left(\mathcal{B}_{T^{\prime}[c \leftrightarrow z] Q}\right.$ inp-cong $\left.\{c\} \mathcal{B}_{T[c \leftrightarrow z] Q}\right)$

$\Rightarrow[\mathcal{B R J C}$ inheritance (Definition 4.7)] 
$T[c \leftrightarrow z] Q \leftarrow_{e c v g} T^{\prime}[c \leftrightarrow z] Q$

[Third case $T[c \hookrightarrow z]]$

$\left[T \leftarrow_{e c v g} T^{\prime} \wedge T[c \hookrightarrow z]\right.$ is deadlock free, substitutability of inheritance (Theorem 4.2)]

$T^{\prime}[c \hookrightarrow z]$ is a deadlock free contract $\wedge$

$\forall\left(t^{\prime}, X\right) \in \mathcal{F}\left(\mathcal{B}_{T^{\prime}}\right), \exists(t, Y) \in \mathcal{F}\left(\mathcal{B}_{T}\right) \bullet t^{\prime} \operatorname{ecvg} t \wedge\left(\begin{array}{c}Y \cap \text { inputs } \supseteq X \cap \text { inputs } \wedge \\ Y \cap \text { outputs } \subseteq X \cap \text { outputs }\end{array}\right)$

$\Rightarrow$ [deadlock free process (ROSCOE, 1998)]

$\forall s \in \Sigma^{*} \bullet\left(s, \Sigma^{\checkmark}\right) \notin \mathcal{F}\left(\mathcal{B}_{T^{\prime}[c \hookrightarrow z]}\right) \wedge$

$\forall\left(t^{\prime}, X\right) \in \mathcal{F}\left(\mathcal{B}_{T^{\prime}}\right), \exists(t, Y) \in \mathcal{F}\left(\mathcal{B}_{T}\right) \bullet t^{\prime}$ ecvg $t \wedge\left(\begin{array}{c}Y \cap \text { inputs } \supseteq X \cap \text { inputs } \wedge \\ Y \cap \text { outputs } \subseteq X \cap \text { outputs }\end{array}\right)$

$\Rightarrow$ [feedback rule (Definition 2.18)]

$\forall s \in \Sigma^{*} \bullet\left(s, \Sigma^{\checkmark}\right) \notin \mathcal{F}\left(\mathcal{B}_{T^{\prime}[c \leftrightarrow z] Q}\right) \wedge \mathcal{B}_{T[c \hookrightarrow z]}=\mathcal{B}_{T} \|_{\{c, z]\}} B U F F_{I O}^{n}\left(R_{I O}^{c \rightarrow z}, R_{I O}^{z \rightarrow c}\right) \wedge$

$\forall\left(t^{\prime}, X\right) \in \mathcal{F}\left(\mathcal{B}_{T^{\prime}}\right), \exists(t, Y) \in \mathcal{F}\left(\mathcal{B}_{T}\right) \bullet t^{\prime} \operatorname{ecvg} t \wedge\left(\begin{array}{c}Y \cap \text { inputs } \supseteq X \cap \text { inputs } \wedge \\ Y \cap \text { outputs } \subseteq X \cap \text { outputs }\end{array}\right)$

$\Rightarrow\left[\begin{array}{l}B U F F_{I O}^{n}\left(R_{I O}^{c \rightarrow z}, R_{I O}^{z \rightarrow c}\right) \text { does not reorder or lose events (Definition 2.14), } \\ \text { buffer tolerance (ROSCOE, 2005) }\end{array}\right]$

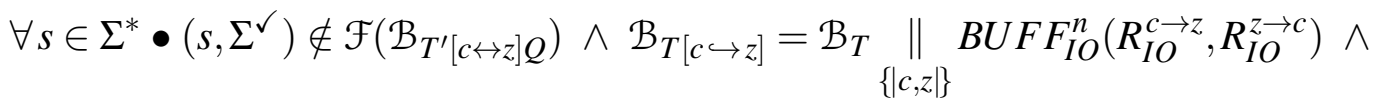

$\forall\left(t^{\prime}, X\right) \in \mathcal{F}\left(\mathcal{B}_{T^{\prime}}\right), \exists(t, Y) \in \mathcal{F}\left(\mathcal{B}_{T}\right) \bullet t^{\prime}$ ecvg $t \wedge\left(\begin{array}{c}Y \cap \text { inputs } \supseteq X \cap \text { inputs } \wedge \\ Y \cap \text { outputs } \subseteq X \cap \text { outputs } \\ \vee(\Sigma Y \subseteq X)\end{array}\right) \wedge$

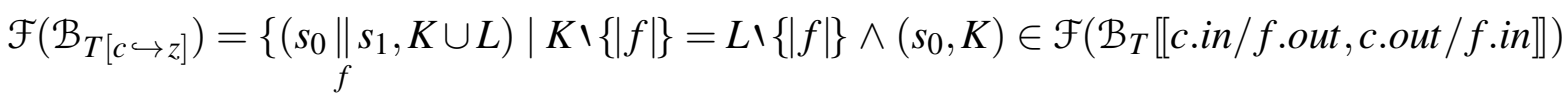

$\left(s_{1}, L\right) \in \mathcal{F}\left(\left(\mathcal{B}_{T} \uparrow z\right)[[z . i n / f . i n, z . o u t / f\right.$.out $\left.\left.]]\right)\right\}[[f$.out $/$ c.in, f.in $/$ c.out,$f / z] \rrbracket \wedge$

$\mathcal{F}\left(\mathcal{B}_{T^{\prime}[c \hookrightarrow z]}\right)=\left\{\left(s_{0} \|_{f} s_{1}, K \cup L\right) \mid K \backslash\{|f|\}=L \backslash\{|f|\} \wedge\left(s_{0}, K\right) \in \mathcal{F}\left(\mathcal{B}_{T^{\prime}}[[c . i n / f\right.\right.$.out, c.out $/$ f.in $\left.]]\right)$

$\left(s_{1}, L\right) \in \mathcal{F}\left(\left(\mathcal{B}_{T^{\prime}} \uparrow z\right)[[z . i n / f . i n, z . o u t / f\right.$. out $\left.\left.]]\right)\right\}[[f$.out $/$ c.in, f.in/c.out,$f / z]]$

$\Rightarrow$ [set theory, failures semantics, rename]

$\forall\left(t^{\prime}, X\right) \in \mathcal{F}\left(\mathcal{B}_{T^{\prime}[c \hookrightarrow z]}\right), \exists(t, Y) \in \mathcal{F}\left(\mathcal{B}_{T[c \hookrightarrow z]}\right) \bullet t^{\prime} \operatorname{ecvg} t \wedge\left(\begin{array}{c}Y \cap \text { inputs } \supseteq X \cap \text { inputs } \wedge \\ Y \cap \text { outputs } \subseteq X \cap \text { out puts }\end{array}\right)$ 
$\Rightarrow\left[\begin{array}{l}\text { default channel congruence (Definition 4.5), input channel congruence (Definition 4.6), } \\ \text { I/O extended convergent behaviour (Definition 4.4) }\end{array}\right]$

$\mathcal{B}_{T^{\prime}[c \hookrightarrow z]} \operatorname{ecvg} \mathcal{B}_{T[c \hookrightarrow z]} \wedge$

$\forall c: \mathcal{C}_{T[c \hookrightarrow z]} \bullet\left(\mathcal{B}_{T^{\prime}[c \hookrightarrow z]}\right.$ def-cong $\left.\{c\} \mathcal{B}_{T[c \hookrightarrow z]}\right) \vee\left(\mathcal{B}_{T^{\prime}[c \hookrightarrow z]}\right.$ inp-cong $\{c c\}$

$\Rightarrow[\mathcal{B R J C}$ inheritance (Definition 4.7)]

$T[c \hookrightarrow z] \triangleleft e c v g T^{\prime}[c \hookrightarrow z]$

[ Fourth case $T\left[c^{\hookrightarrow} \rightarrow z\right]$ ] It is almost identical to the third part.

\section{Checking convergence via refinement}

Behaviour convergence (Definitions 4.2 and 4.4) is the backbone of this work: it is used to define inheritance and an aspect-oriented theory for $\mathcal{B R J \mathcal { C }}$ (Chapter 6). Therefore, we must have an automated strategy to check whether two I/O processes are related by convergence, especially for component specifications with large state space. We start addressing this issue by choosing FDR3 (Failures-Divergence Refinement) (GIBSON-ROBINSON et al., 2014) as the model-checker to carry out the analysis; it seems a natural choice given the widespread use of FDR3 both in academy and industry, which makes it a de facto standard tool for analysing CSP specifications. Its method of establishing whether a property holds is to check for the refinement between CSP specifications, internally represented by labelled transition systems.

The problem is that we have not a built-in function to encode the convergence relations (Definitions 4.2 and 4.4) in FDR3. Our strategy DIHEGO (2015) is to construct, for each relation, a verification strategy of the form:

$$
\begin{aligned}
& P^{\prime} \text { io_cvg } P \Longleftrightarrow \text { Tester_cvg }(P) \sqsubseteq_{\mathrm{F}} P^{\prime} \\
& P^{\prime} \text { io_ecvg } P \Longleftrightarrow \text { Tester_ecvg }(P) \sqsubseteq_{\mathrm{F}} P^{\prime}
\end{aligned}
$$

To understand how the parametrised processes Tester_cvg $(P)$ and Tester_ecvg $(P)$ can be constructed, we need to present some additional background on failures refinement, convergence and $\mathrm{I} / \mathrm{O}$ process alternative representations. Consider $P$ an $\mathrm{I} / \mathrm{O}$ process, then $c v g^{+} P$ stands for a set of I/O processes such that: $\forall P^{\prime} \in c v g^{+} P \mid P^{\prime}$ io_cvg $P$; it contains every I/O processes convergent to $P$, including $P$ itself. The set $c v g^{+} P$ is infinite, which makes its use prohibitive for any implementation that aims to traverse it. A finite subset of $c v g^{+} P$ is given by $c v g^{+n} P$, which stands for the $P$ convergent processes, whose depth differ from that of $P$ by at most $n \in \mathbb{N}$, where $\mathrm{I} / \mathrm{O}$ process depth is given by the greatest number of events that makes a process return, only once, to its initial state or, by considering its LTS, the maximum number of transitions (labelled with visible events) from the initial state to itself. An I/O process depth can be equivalently expressed in two ways: (a) in terms of traces and failures-semantics or (b) based on its LTS 
representation, where $P \stackrel{t}{\Longrightarrow} P$ means that the I/O process $P$ comes back to its initial state after the trace $t$ :

(a) $\operatorname{depth}(P)=\max \left\{\# t \mid t \in \mathcal{T}(P) \wedge P \sqsubseteq_{\mathrm{F}} P / t \wedge\left(\nexists s<t \mid P \sqsubseteq_{\mathrm{F}} P / s\right)\right\}$

(b) $\operatorname{depth}(P)=\max \{\# t \mid P \stackrel{t}{\Longrightarrow} P \wedge(\nexists s<t \mid P \stackrel{s}{\Longrightarrow} P)\}$

The core of our strategy is to build a CSP process $L U B$, such that every member $Q$ of $c v g^{+n} P$ refines it, $L U B \sqsubseteq_{\mathrm{F}} Q$. It means that the process $L U B$ is the least upper bound (ENDERTON, 1977; ROSCOE, 1998) of the set $c v g^{+n} P \cup\{L U B\}$ under CSP failures refinement relation $\left(\sqsubseteq_{\mathrm{F}}\right)$. Our intention is to construct Tester_cvg $(P)$ to be failures equivalent to $L U B$, Tester_cvg $(P) \equiv_{\mathrm{F}} L U B$. Therefore, to verify if a process $P^{\prime}$ is convergent to $P$, i.e., if $P^{\prime}$ belongs to $c v g^{+n} P$, one must verify if Tester_cvg $(P) \sqsubseteq_{\mathrm{F}} P^{\prime}$; this is the kind of verification where FDR3 exceeds in efficiency. The same reasoning applies to the extended convergence. The next section details how we construct Tester_ecvg $(P)$ and Tester_cvg $(P)$ for an I/O process $P$.

\section{Building Tester_cvg(ecvg)}

Let $P$ and $P^{\prime}$ be $\mathrm{I} / \mathrm{O}$ processes that differ in depth by $n$. To test whether $P^{\prime}$ io_cvg $P$, we must build from $P$ a new process (Tester_cvg $(P)$ ), which must be able to do at most $n$ new-in-context inputs in every state of $P$. Such a process is, by Definition 4.2, convergent to $P$. As we said before, any process convergent to $P$ (which differs in depth by at most $n$ ) must refine in failures Tester_cvg $(P)$. An important practical question to build Tester_cvg $(P)$ is to compute the new-in-context inputs for any state of $P$. Given a state of $P$, the easiest way of computing the new-in-context inputs available is to know which inputs can be accepted in this state; the result will be its complement. The problem is that CSP does not have a native mechanism for backtracking a process execution: we cannot synchronise on an event and then go back to the state before it. A complicating factor is that we are dealing with new-in-context events, not new-in-alphabet events; if this was the case, alphabetised parallelism and hiding could be sufficient to compare the behaviours of the two components modulo the new events, as already demonstrated by (WEHRHEIM, 2002). To circumvent this problem we define an alternative representation for I/O processes, with a finite LTS. We serialise an I/O process as a sequence of tuples of the form $\left(e v, a \_e v, l\right)$, for a particular state, where the elements of the tuple stand for: the event $e v$ is possible from this state and if it happens the next state can accept the events in the sequence $a \_e v$, which is in the level $l+1$. The initial state's level is zero; each subsequent state has the level of its immediate predecessor increased by one. We use sequences instead of sets mainly for two reasons: firstly, if an I/O process offers an internal choice on the same output event, this information is lost by the set uniqueness property, which is not the case for sequences and, secondly, because the iteration/removal/partition operations on sequences are more directly 
available in CSP than their counterparts on sets.

Let us consider a practical example on how an I/O process can be serialised. For this consider the process $P$ in Figure 4.1. We define two special events start and end; these are used only as mark events, and will not be part of the Tester_* behaviour, but will play a role in its construction: start indicates that a process is ready to engage by offering its initial events; end marks the state where it is ready to come back to its initial state. Line 1 of P_serial (see below) indicates that $P$ can, initially (state level zero) accept c. in.v. 1 or C. in.v.2; if c. in.v. 1 happens (line 2, state level 1) then it can output (non-deterministically) c . out . v. 1 or c.out.v.2; if it outputs C.out.v.1 (line 3, state level 2) then it can only go back to its initial state (end). Note that we follow a nested-structural pattern, which allows us to backtrack an $\mathrm{I} / \mathrm{O}$ process by traversing its serial representation in a recursive manner.

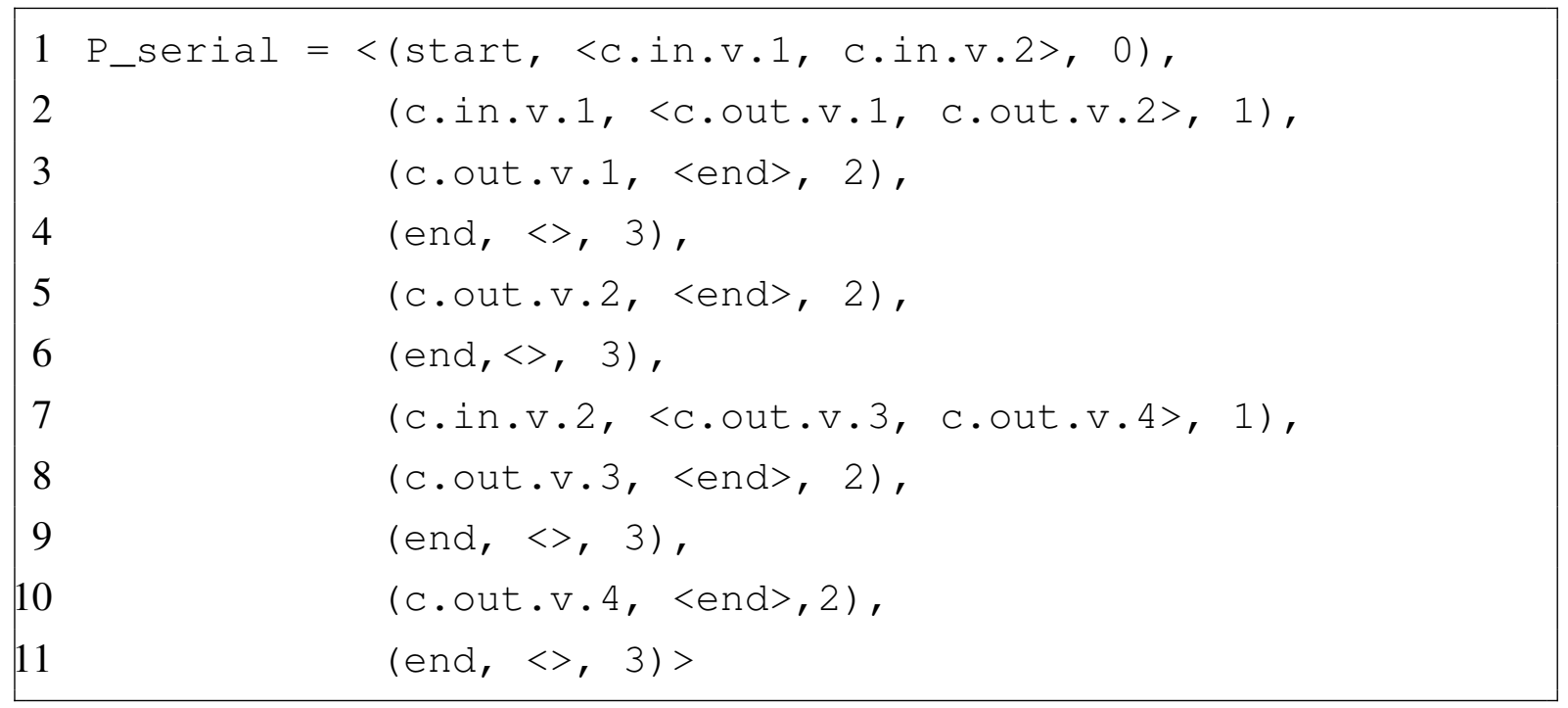

A question that arises is how to deal with parallelism in such a representation. As we have discussed in Chapter 2, any parallel process has a unique sequential representation in terms of $\rightarrow, \sqcap$ and $\square$ operators (ROSCOE, 1998).

We have decomposed Tester_cvg into a set of helper processes and their associated functions. The process $E X E C(e v s, n)$ offers in internal choice the events evs, up to $n$ times.

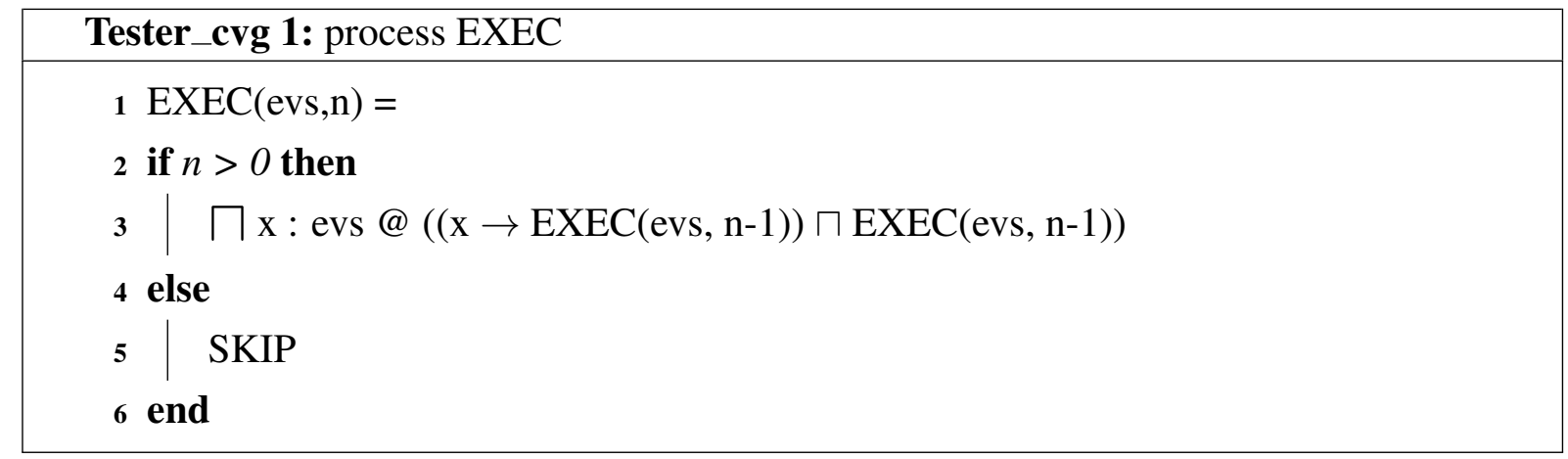

The process EXEC $D$ is quite similar to EXEC but differs as it offers evs in external 
choice.

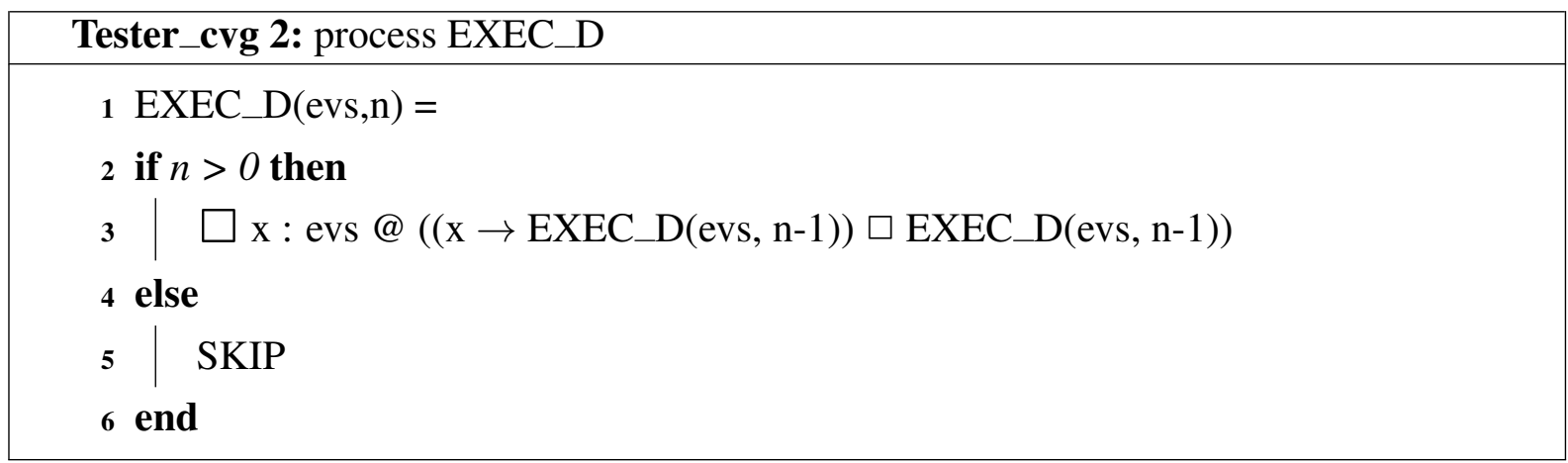

The process $E X E C \_Q$ combines EXEC and EXEC_D in parallel. Let evs 1 and evs 2 be sets of events such that evs $2 \subseteq e v s 1$, then $E X E C$ offers evs $1 \backslash e v s 2$ in internal choice and EXEC_D offers evs 1 in external choice up to $n$ times, synchronising on each step on the set evs1levs2.

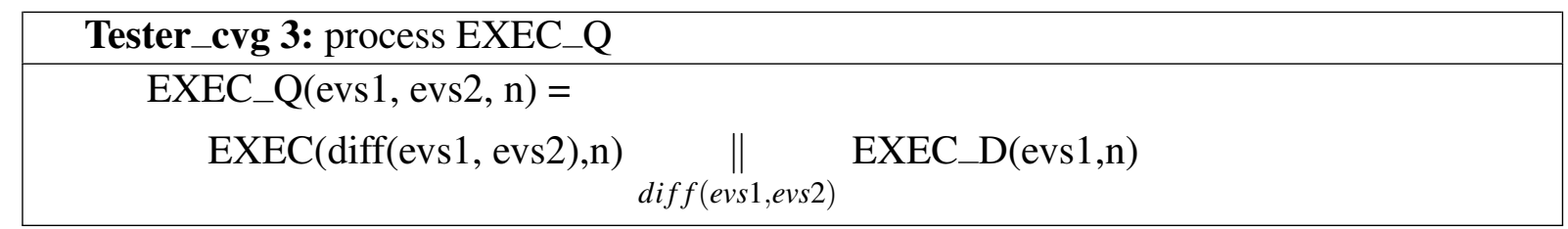

Given a 3-tuple of an I/O process serialised representation, the functions $e 1, e 2$ and $e 3$ are designed to access its respective positions. We declare the aforementioned channels start and $e n d$ and define the function $\operatorname{subset}(s 1, s 2)$ to check whether $s 1$ is a subset of $s 2$.

$$
\begin{aligned}
& \text { channel start, end } \\
& \mathrm{e} 1\left(\left(\mathrm{e},{ }_{-},-\right)\right)=\mathrm{e} \\
& \mathrm{e} 2\left(\left({ }_{-}, \mathrm{e},-\right)\right)=\mathrm{e} \\
& \mathrm{e} 3((-,,, \mathrm{e}))=\mathrm{e} \\
& \text { subset }(\mathrm{s} 1, \mathrm{~s} 2)=\operatorname{empty}(\operatorname{diff}(\mathrm{s} 1, \mathrm{~s} 2))
\end{aligned}
$$

The branch function yields a local view of a serialized I/O process: giving some event $k e y$ at a particular level $l$, it provides the tuples (a branch) reached from key (at level $l$ ) until the end mark (the event end). For example, considering P_serial, if we ask for the branch started with (a) the event c.in.v.1 at the level 1 or (b) the event c.out.v.2 at the level 2, we obtain, respectively:

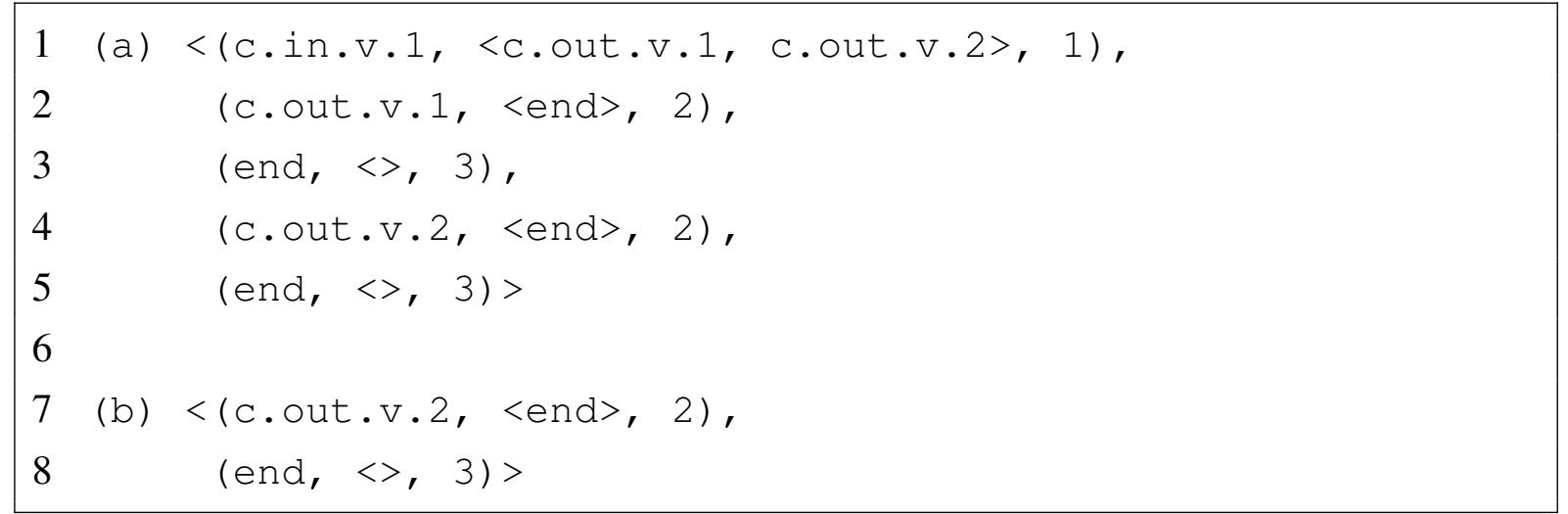

It works finding the tuples, in a sequence $s$, whose events are offered together, by looking 
for one of them, say key, in a specific level $l$. If $s$ is empty (line 2) we have finished the search and the result remains unchanged (line 3). Otherwise, we check if the current tuple has the event at the level we are looking for (line 5); if it is the case, we append this tuple to the result and call branch recursively, passing to it the remainder of the sequence and setting the parameter $b$ (marking the first discovery of a tuple with key) to true (line 6).

If we have found the key event ( $b$ is true, line 8 ), we must check if we have not reached another branch of the process LTS at the same level (line 9), which marks the end of our search (line 10), otherwise we append such a tuple to the result and call branch to the rest of the sequence (line 12). If we have not yet found the key, we maintain the result unchanged and recursively call branch to the rest of the sequence (line 15).

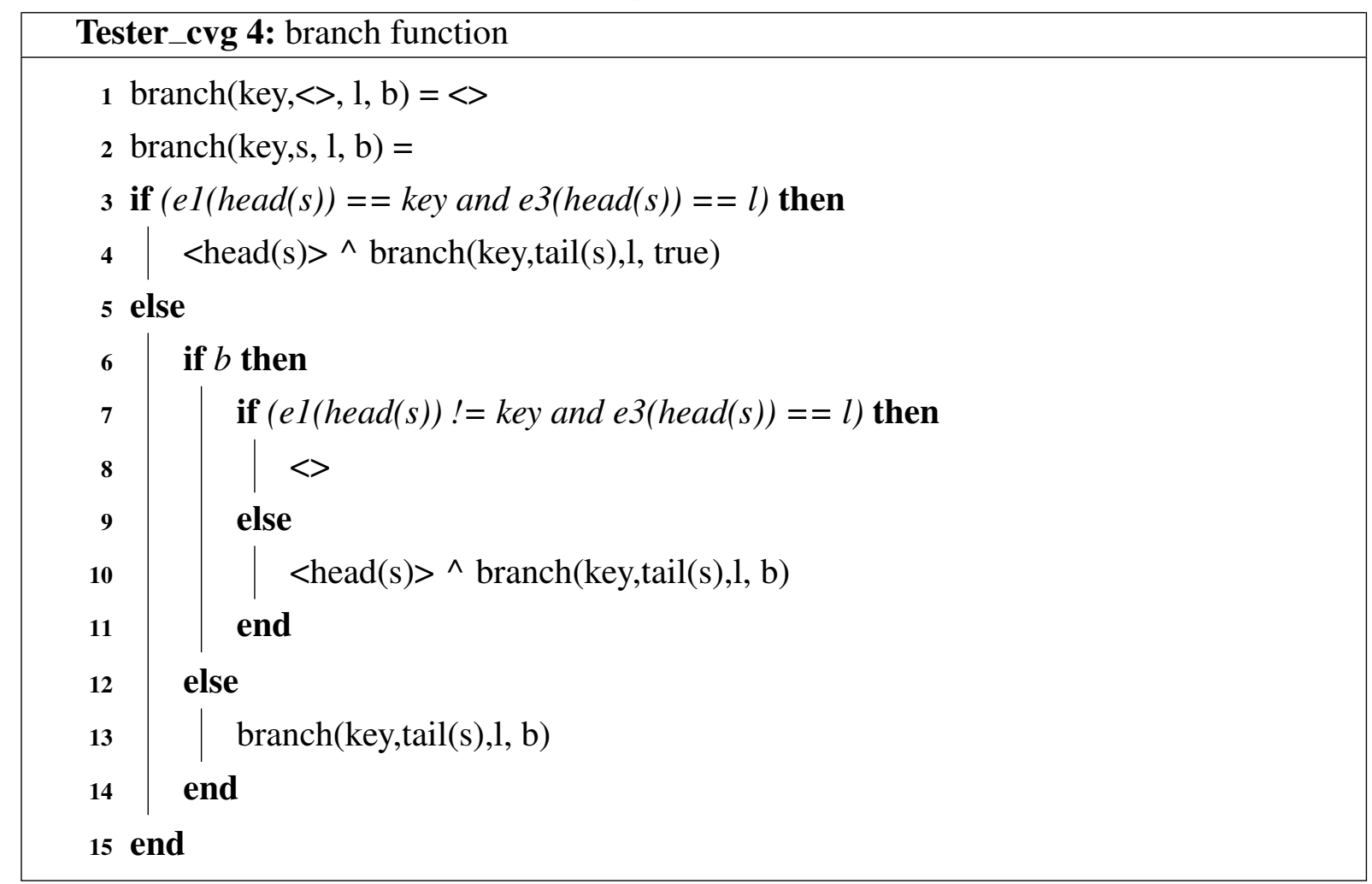

The process $C V G \_B U I L D E R$ coordinates the helper processes we have seen in the work of building Tester_cvg $(P)$ for an I/O processes $P$. Let $s r c$ be the serialised representation of $P$ and $c r t$ the serialisation of a $P$ 's current execution branch. Both are parameters of the $C V G \_B U I L D E R$ process and are equal initially. If we have found the end of a branch (line 2), $C V G \_B U I L D E R$ returns to $P$ 's initial state by making $c r t$ equals to $\operatorname{src}$ (line 3 ) again. Otherwise it must offer an external choice between inputs (line 5) or an internal choice between outputs (line 10). In the first case, it has the chance to execute up to $n(n=G A P \mid G A P \in \mathbb{N})$ new-in-context inputs (line 6) before converging to $P$ (lines 8 and 9 ). Note that only new in-context-inputs (inputs $\backslash \operatorname{set}(e 2($ head $(\mathrm{crt})))$ are allowed to be executed non-deterministically, as we cannot violate the external choice between expected inputs set $(e 2(h e a d(c r t))$; therefore we use the helper process $E X E C \_Q$ to do such a task. In the second case (line 10), before an internal choice between outputs is offered, $C V G \_B U I L D E R$ can do up to $n$ inputs (line 11) before converging 
to $P$ (lines 12 and 13 ).

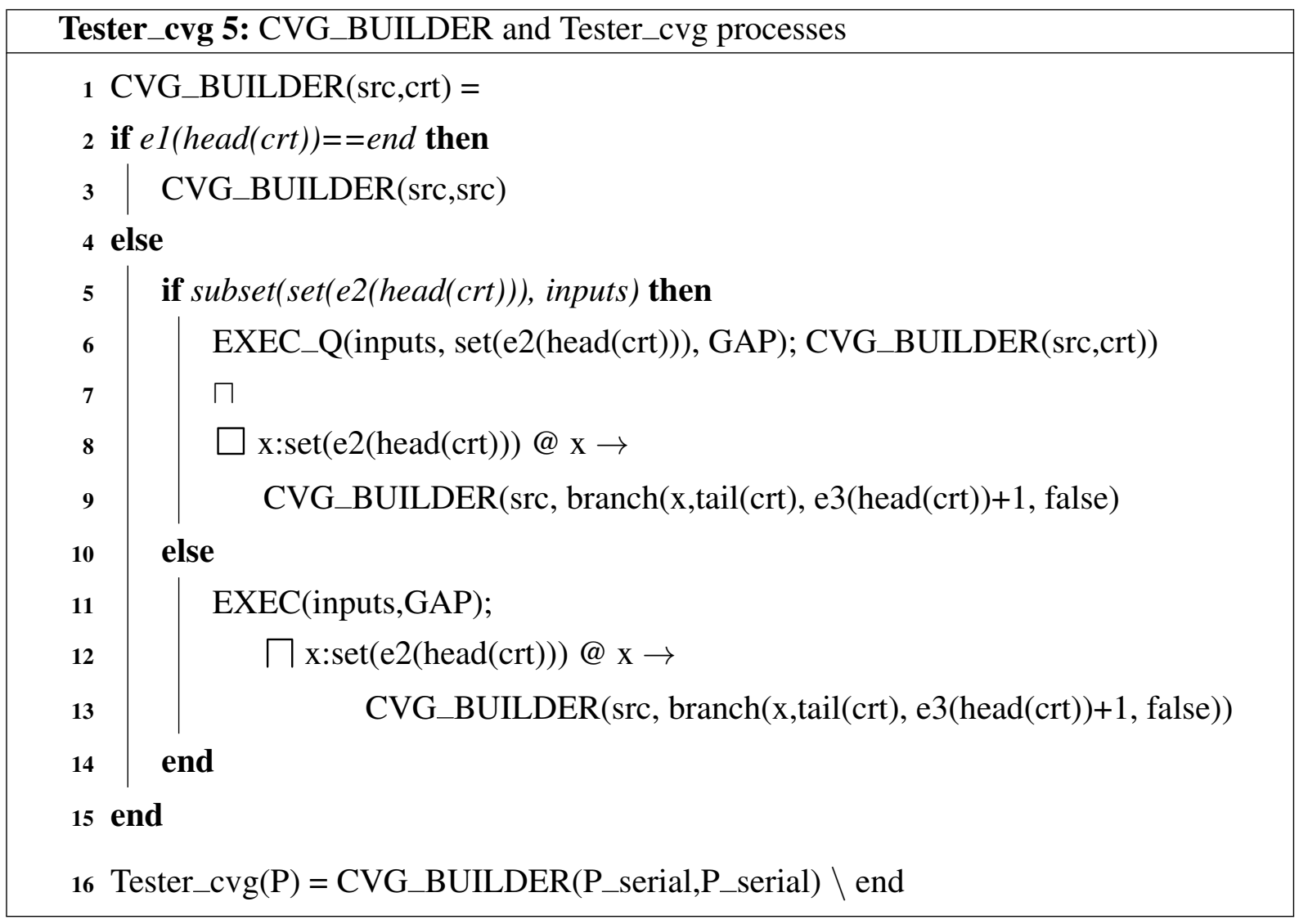

Therefore, if we consider the CSP processes $P$ and $P^{\prime}$, whose labelled transition systems are depicted in Figure 4.1, the following FDR3 assertions hold. The second assertion is particularly interesting; it is a consequence of Lemma 4.1, from where we know $P$ io_cvg $P$ because $P \sqsubseteq \mathrm{F} P$. Otherwise, if we consider the I/O processes of Figure 4.2, the first FDR assertion, as we expected, evaluates to false.

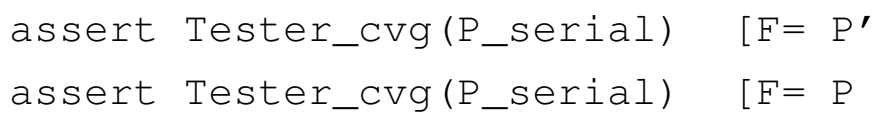

Theorem 4.4 (Tester_cvg soundness). Consider $P$ and $P^{\prime}$ two I/O processes, such that $P \_s e r i a l$ stands for the serialised representation of $P$. If $P^{\prime}$ ecvg $P$ then the following refinement holds Tester_cvg $\left(P \_\right.$serial $) \sqsubseteq_{\mathrm{F}} P^{\prime}$.

Proof. In this proof we use some equivalences derived from the denotational semantics of CSP, discussed in Section 2.1, and from its algebraic semantics, defined by (ROSCOE, 1998).

(a) $\operatorname{EXEC}($ evs, 0$)=S K I P$ $\langle$ Definition of EXEC (Tester_cvg 1)〉 $\operatorname{EXEC}(e v s, 1)=\sqcap x: e v s @((x \rightarrow E X E C(e v s, 0)) \sqcap \operatorname{EXEC}(e v s, 0))$ 
(b) $\operatorname{EXEC}($ evs, 1$)=\left(\prod x:\right.$ evs @ $\left.(x \rightarrow S K I P)\right) \sqcap S K I P$

$\langle\sqcap-\sqcap$-distributivity $\rangle$

$\operatorname{EXEC}(e v s, 2)=\sqcap x: e v s @((x \rightarrow \operatorname{EXEC}($ evs, 1$)) \sqcap \operatorname{EXEC}($ evs, 1$))$

$\langle$ Definition of EXEC (Tester_cvg 1)〉

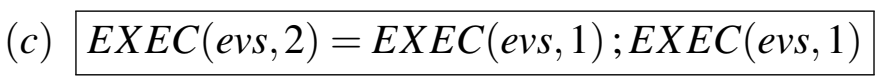

$\langle(b) ; \sqcap-П$-distributivity $\rangle$

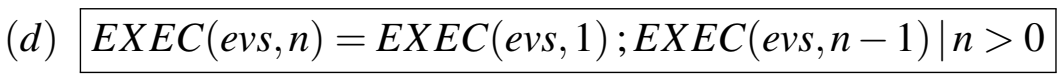

The following equivalences verify for the process $E X E C D$ :

(e) $E X E C D(e v s, 0)=S K I P$

$\langle$ Definition of EXEC_D (Tester_cvg 2) >

$E X E C \_D(e v s, 1)=\square x:$ evs @ $\left(\left(x \rightarrow E X E C \_D(e v s, 0)\right) \square E X E C \_D(e v s, 0)\right)$

$\langle$ Definition of EXEC_D (Tester_cvg 2) $\rangle$

$E X E C \_D($ evs, 1$)=\square x:$ evs @ $((x \rightarrow S K I P) \square S K I P)$

$(f) \quad E X E C \_$evs, 1$)=(\square x:$ evs @ $(x \rightarrow S K I P)) \square S K I P$

$\langle\square-\square$-distributivity $\rangle$

$E X E C \_D(e v s, 2)=\square x:$ evs @ $\left(\left(x \rightarrow E X E C \_D(e v s, 1)\right) \square E X E C \_D(e v s, 1)\right)$

$\langle$ Definition of EXEC_D (Tester_cvg 2) $\rangle$

(g) $E X E C \_D(e v s, 2)=E X E C \_D(e v s, 1) ; E X E C \_D(e v s, 1)$

$\langle(f) ; \square-\square$-distributivity $\rangle$

(h) $E X E C \_D($ evs,$n)=E X E C \_D($ evs, 1$) ; E X E C \_D($ evs,$n-1) \mid n>0$

$\langle(f) ;(g)\rangle$

The following equivalences and refinements verify for the process $E X E C \_Q$ :

$E X E C \_Q(e v s 1, e v s 2,0)=E X E C($ evs $1 \backslash e v s 2,0) \|_{\text {evs } 1 \backslash e v s 2} E X E C \_D(e v s 1,0)$

$\langle$ Definition of EXEC_Q $($ Tester_cvg 3) $\rangle$ 
$E X E C_{-} Q($ evs $1, e v s 2,0)=S K I P \underset{\text { evs } 1 \backslash e v s 2}{\|} S K I P$

(i) $E X E C \_Q(e v s 1, e v s 2,0)=S K I P$

$\langle S K I P ; \|\rangle$

$E X E C \_Q(e v s 1, e v s 2,1)=\left(E X E C(\right.$ evs $1 \backslash e v s 2,1) \|_{\text {evs } 1 \backslash e v s 2} E X E C \perp D($ evs 1,1$\left.)\right)$

$\langle$ Definition of EXEC_Q $($ Tester_cvg 3) $\rangle$

$E X E C \_Q(e v s 1, e v s 2,1)=$

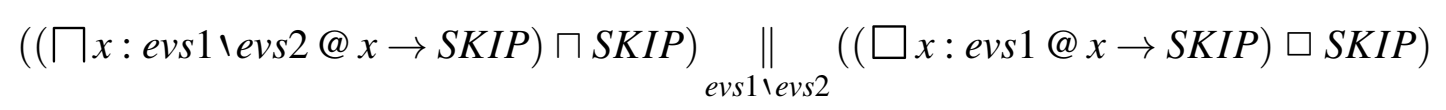

$(j)$

$$
\begin{aligned}
& \text { EXEC_Q }(\text { evs } 1, \text { evs } 2,1)= \\
& ((\square x: \text { evs } 2 @ x \rightarrow(S K I P \sqcap S T O P)) \square \text { SKIP }) \\
& \sqcap \quad((\square x: \text { evs } 2 @ x \rightarrow S K I P) \square \text { SKIP }) \\
& \sqcap \quad((\square x: \text { evs1 } 1 \text { evs } 2 @ x \rightarrow S K I P) \square \quad(\square x: \text { evs } 2 @ x \rightarrow \text { STOP }))
\end{aligned}
$$

$\langle$ SKIP; $\sqcap-П$-distributivity; $\square-\square$-distributivity; $\|\rangle$

(k) $E X E C_{-} Q($ evs $1, e v s 2,2) \sqsubseteq_{\mathrm{F}} E X E C_{-} Q($ evs $1, e v s 2,1) ; E X E C_{-} Q($ evs $1, e v s 2,1)$

$$
\langle(j) ; S K I P ; \| ; \sqcap ; \square\rangle
$$

(l) $E X E C_{-} Q(e v s 1, e v s 2, n) \sqsubseteq_{\mathrm{F}} E X E C_{-} Q(e v s 1, e v s 2,1) ; E X E C_{-} Q(e v s 1, e v s 2, n-1) \mid n>0$

$$
\langle(j) ;(k)\rangle
$$

In this proof we follow the behaviour of Tester_cvg $\left(P_{\_}\right.$serial $)$, which is defined as the CSP process $C V G \_B U I L D E R\left(P \_\right.$serial, $P \_$serial) (Listing 6). Our strategy is to prove that $P^{\prime}$ refines $C V G \_B U I L D E R\left(P \_s e r i a l, P \_s e r i a l\right)$, from its initial state to any subsequent one; otherwise, $P^{\prime}$ is not $\mathrm{I} / \mathrm{O}$ convergent to $P$. The I/O processes $P^{\prime}$ and $P$ can have the same depth, or differ from one up to $n$, so $G A P \in\{0 \ldots n \mid n \in \mathbb{N}\}$.

[First part]

$\left[G A P=0 \wedge C V G \_B U I L D E R\left(P \_s e r i a l, P \_\right.\right.$serial $)($Listing 6) $\wedge e 1$ definition (Listing 4) $]$

$e 1\left(\right.$ head $\left(P \_\right.$serial $\left.)\right) \neq$end

$\Rightarrow[\operatorname{initials}(P) \subseteq$ inputs $] \quad$ (First case) 
$C V G \_B U I L D E R\left(P \_\right.$serial,$P \_$serial $)=$

EXEC_Q $\left(\right.$ inputs, set $\left(e 2\left(\right.\right.$ head $\left(P \_\right.$serial $\left.\left.\left.\left.)\right)\right), 0\right) ; C V G \_B U I L D E R\left(P \_s e r i a l, P \_s e r i a l\right)\right)$

$\sqcap \quad\left(\square x: \operatorname{set}\left(e 2\left(\operatorname{head}\left(P \_\right.\right.\right.\right.$serial $\left.\left.)\right)\right) @ x \rightarrow$

$C V G \_B U I L D E R\left(P \_\right.$serial, $\operatorname{branch}\left(x, \operatorname{tail}\left(P \_\right.\right.$serial $), e 3\left(\right.$ head $\left.\left(P \_s e r i a l\right)\right)+1$, false $\left.)\right)$

$\equiv[$ equivalence $(i)]$

$C V G \_B U I L D E R\left(P \_\right.$serial,$P \_$serial $)=$

SKIP $; C V G \_B U I L D E R\left(P \_\right.$serial,$P \_$serial $)$

$\sqcap \quad\left(\square x: \operatorname{set}\left(e 2\left(\right.\right.\right.$ head $\left(P \_\right.$serial $\left.\left.)\right)\right) @ x \rightarrow$

$C V G \_B U I L D E R\left(P \_\right.$serial, $\operatorname{branch}\left(x, \operatorname{tail}\left(P \_\right.\right.$serial $), e 3\left(\right.$ head $\left(P \_\right.$serial $\left.)\right)+1$, false $\left.)\right)$

$\equiv[$ SKIP semantics $($ Section 2.1) $\wedge \quad ;$ unit law $($ ROSCOE, 1998) $]$

$C V G \_B U I L D E R\left(P \_\right.$serial,$P \_$serial $)=$

$C V G \_B U I L D E R\left(P \_s e r i a l, P \_s e r i a l\right) \quad \square \quad\left(\square x: \operatorname{set}\left(e 2\left(\right.\right.\right.$ head $\left.\left.\left(P \_s e r i a l\right)\right)\right) @ x \rightarrow$ $C V G \_B U I L D E R\left(P \_\operatorname{serial}, \operatorname{branch}\left(x, \operatorname{tail}\left(P \_\right.\right.\right.$serial $), e 3\left(\right.$ head $\left(P \_\right.$serial $\left.)\right)+1$, false $\left.)\right)$

$\Rightarrow$ [failures semantics (Section 2.1.2) $\wedge \quad \square, \square$ semantics (ROSCOE, 1998)]

$\left(\langle\rangle, \Sigma \backslash \operatorname{set}\left(e 2\left(\right.\right.\right.$ head $\left(P \_\right.$serial $\left.\left.\left.)\right)\right)\right) \in \mathcal{F}\left(C V G \_B U I L D E R\left(P \_\right.\right.$serial,$P \_$serial $\left.)\right)$

$\Rightarrow\left[P^{\prime}\right.$ ecvg $P($ Definition 4.2$\left.)\right]$

$\left(\langle\rangle, \Sigma \backslash \operatorname{set}\left(e 2\left(\right.\right.\right.$ head $\left.\left.\left.\left(P \_s e r i a l\right)\right)\right)\right) \in \mathcal{F}\left(C V G \_B U I L D E R\left(P \_\right.\right.$serial,$\left.\left.P \_s e r i a l\right)\right) \wedge$

$(\langle\rangle, X) \in \mathcal{F}\left(P^{\prime}\right) \mid X \subseteq \Sigma \Rightarrow$

$\exists(\langle\rangle, Y) \in \mathcal{F}(P) \mid Y \cap$ inputs $\supseteq X \cap$ inputs $\wedge Y \cap$ outputs $\subseteq X \cap$ outputs

$\Rightarrow\left[\begin{array}{l}\left.\operatorname{set}\left(e 2\left(\text { head }\left(P \_ \text {serial }\right)\right)\right)\right)=\text { initials }(P) \subseteq \text { inputs } \wedge G A P=0 \\ \text { input determinism }(\text { Definition } 2.4) \wedge \text { set theory }\end{array}\right]$

$\left(\langle\rangle, \Sigma \backslash \operatorname{set}\left(e 2\left(\right.\right.\right.$ head $\left(P \_\right.$serial $\left.\left.\left.)\right)\right)\right) \in \mathcal{F}\left(C V G \_B U I L D E R\left(P \_\right.\right.$serial,$\left.\left.P \_s e r i a l\right)\right), \mathcal{F}(P), \mathcal{F}\left(P^{\prime}\right)$

$\Rightarrow[$ initials $(P) \nsubseteq$ inputs $\wedge$ GAP $=0] \quad$ (Second case)

$\left(\langle\rangle, \Sigma \backslash \operatorname{set}\left(e 2\left(\operatorname{head}\left(P \_\right.\right.\right.\right.$serial $\left.\left.\left.)\right)\right)\right) \in \mathcal{F}\left(C V G \_B U I L D E R\left(P \_\right.\right.$serial,$P \_$serial $\left.)\right), \mathcal{F}(P), \mathcal{F}\left(P^{\prime}\right) \quad \vee$

$C V G \_B U I L D E R\left(P \_\right.$serial,$P \_$serial $)=$

EXEC(inputs, 0$) ;\left(П x: \operatorname{set}\left(e 2\left(\right.\right.\right.$ head $\left(P \_\right.$serial $\left.\left.)\right)\right) @ x \rightarrow$

$C V G \_B U I L D E R\left(P \_\operatorname{serial}, \operatorname{branch}\left(x, \operatorname{tail}\left(P \_\right.\right.\right.$serial $), e 3\left(\right.$ head $\left(P \_\right.$serial $\left.)\right)+1$, false $\left.\left.)\right)\right)$ 
$\equiv[$ equivalence $(a)]$

$\left(\langle\rangle, \Sigma \backslash \operatorname{set}\left(e 2\left(\right.\right.\right.$ head $\left(P \_\right.$serial $\left.\left.\left.)\right)\right)\right) \in \mathcal{F}\left(C V G \_B U I L D E R\left(P \_s e r i a l, P \_s e r i a l\right)\right), \mathcal{F}(P), \mathcal{F}\left(P^{\prime}\right) \quad \vee$

$C V G \_B U I L D E R\left(P \_\right.$serial,$P \_$serial $)=$

SKIP; $\left(П x: \operatorname{set}\left(e 2\left(\right.\right.\right.$ head $\left.\left.\left(P \_s e r i a l\right)\right)\right) @ x \rightarrow$

$C V G \_B U I L D E R\left(P \_s e r i a l, \operatorname{branch}\left(x, \operatorname{tail}\left(P \_\right.\right.\right.$serial $), e 3\left(\right.$ head $\left(P \_\right.$serial $\left.)\right)+1$, false $\left.\left.)\right)\right)$

$\equiv[S K I P$ semantics $($ Section 2.1) $\wedge ;$ unit law $($ ROSCOE, 1998) $]$

$\left(\langle\rangle, \Sigma \backslash \operatorname{set}\left(e 2\left(\right.\right.\right.$ head $\left(P \_\right.$serial $\left.\left.\left.)\right)\right)\right) \in \mathcal{F}\left(C V G \_B U I L D E R\left(P \_\right.\right.$serial,$P \_$serial $\left.)\right), \mathcal{F}(P), \mathcal{F}\left(P^{\prime}\right) \quad \vee$

$C V G \_B U I L D E R\left(P \_\right.$serial,$P \_$serial $)=\left(П x: \operatorname{set}\left(e 2\left(\right.\right.\right.$ head $\left(P \_\right.$serial $\left.\left.)\right)\right) @ x \rightarrow$

$C V G \_B U I L D E R\left(P \_\operatorname{serial}, \operatorname{branch}\left(x, \operatorname{tail}\left(P \_\right.\right.\right.$serial $), e 3\left(\right.$ head $\left(P \_\right.$serial $\left.)\right)+1$, false $\left.\left.)\right)\right)$

$\Rightarrow$ [failures semantics (Section 2.1.2) $\wedge \quad \sqcap$ semantics (ROSCOE, 1998)

$\left(\langle\rangle, \Sigma \backslash \operatorname{set}\left(e 2\left(\right.\right.\right.$ head $\left(P \_\right.$serial $\left.\left.\left.)\right)\right)\right) \in \mathcal{F}\left(C V G \_B U I L D E R\left(P \_\right.\right.$serial,$P \_$serial $\left.)\right), \mathcal{F}(P), \mathcal{F}\left(P^{\prime}\right) \quad \vee$

$(\langle\rangle, \Sigma \backslash e) \in \mathcal{F}\left(C V G \_B U I L D E R\left(P \_\right.\right.$serial,$P \_$serial $\left.)\right) \mid e \in \operatorname{set}\left(e 2\left(\right.\right.$ head $\left(P \_\right.$serial $\left.\left.)\right)\right)$

$\Rightarrow\left[P^{\prime} \operatorname{ecvg} P(\right.$ Definition 4.2$\left.)\right]$

$\left(\langle\rangle, \Sigma \backslash \operatorname{set}\left(e 2\left(\right.\right.\right.$ head $\left(P \_\right.$serial $\left.\left.\left.)\right)\right)\right) \in \mathcal{F}\left(C V G \_B U I L D E R\left(P \_\right.\right.$serial,$P \_$serial $\left.)\right), \mathcal{F}(P), \mathcal{F}\left(P^{\prime}\right) \quad \vee$

$(\langle\rangle, \Sigma \backslash e) \in \mathcal{F}\left(C V G \_B U I L D E R\left(P \_\right.\right.$serial,$P \_$serial $\left.)\right) \mid e \in \operatorname{set}\left(e 2\left(\right.\right.$ head $\left(P \_\right.$serial $\left.\left.)\right)\right)$

$\wedge(\langle\rangle, X) \in \mathcal{F}\left(P^{\prime}\right) \mid X \subseteq \Sigma \Rightarrow$

$\exists(\langle\rangle, Y) \in \mathcal{F}(P) \mid Y \cap$ inputs $\supseteq X \cap$ inputs $\wedge Y \cap$ outputs $\subseteq X \cap$ outputs

$\Rightarrow\left[\begin{array}{l}\left.\operatorname{set}\left(e 2\left(\text { head }\left(P_{\_} \text {serial }\right)\right)\right)\right)=\text { initials }(P) \subseteq \text { out puts } \wedge G A P=0 \\ \text { strong output decisiveness }(\text { Definition } 2.4) \wedge \text { set theory }\end{array}\right]$

$\left(\langle\rangle, \Sigma \backslash \operatorname{set}\left(e 2\left(\right.\right.\right.$ head $\left(P \_\right.$serial $\left.\left.\left.)\right)\right)\right) \in \mathcal{F}\left(C V G \_B U I L D E R\left(P \_\right.\right.$serial,$P \_$serial $\left.)\right), \mathcal{F}(P), \mathcal{F}\left(P^{\prime}\right) \vee \vee$

$(\langle\rangle, \Sigma \backslash e) \in \mathcal{F}\left(C V G \_B U I L D E R\left(P \_\right.\right.$serial,$P \_$serial $\left.)\right), \mathcal{F}(P), \mathcal{F}\left(P^{\prime}\right) \mid e \in \operatorname{set}\left(e 2\left(\right.\right.$ head $\left(P \_\right.$serial $\left.\left.)\right)\right)$

$\Rightarrow\left[\begin{array}{l}\text { induction over } C V G \_B U I L D E R\left(P \_ \text {serial }, P \_ \text {serial }\right) \\ \text { structure until } e 1(\text { head }(\text { crt }))==\text { end }(\text { Listing } 6)\end{array}\right]$

$\forall(t, X) \in \mathcal{F}\left(P^{\prime}\right) \Rightarrow(t, X) \mathcal{F}\left(C V G \_B U I L D E R\left(P \_\right.\right.$serial,$P \_$serial $\left.)\right) \mid G A P=0$ 
$\equiv[$ failures semantics $]$

$C V G \_B U I L D E R\left(P \_\right.$serial,$P \_$serial $) \sqsubseteq_{\mathrm{F}} P^{\prime} \mid G A P=0$

\section{[Second part]}

$\left[G A P=1 \wedge C V G \_B U I L D E R\left(P \_\right.\right.$serial,$P \_$serial $)($Listing 6$) \wedge e 1$ definition (Listing 4$\left.)\right]$

$e 1\left(\right.$ head $\left.\left(P \_s e r i a l\right)\right) \neq$ end

$\Rightarrow[$ initials $(P) \subseteq$ inputs $] \quad$ (First case)

$C V G \_B U I L D E R\left(P \_\right.$serial,$P \_$serial $)=$

EXEC_Q $\left(\right.$ inputs, set $\left(e 2\left(\right.\right.$ head $\left.\left.\left.\left.\left(P \_s e r i a l\right)\right)\right), 1\right) ; C V G \_B U I L D E R\left(P \_s e r i a l, P \_s e r i a l\right)\right)$

$\sqcap \quad\left(\square x: \operatorname{set}\left(e 2\left(\operatorname{head}\left(P \_\right.\right.\right.\right.$serial $\left.\left.)\right)\right) @ x \rightarrow$

$C V G \_B U I L D E R\left(P \_\right.$serial, $\operatorname{branch}\left(x, \operatorname{tail}\left(P \_\right.\right.$serial $), e 3\left(\right.$ head $\left(P \_\right.$serial $\left.\left.\left.)\right)+1, f a l s e\right)\right)$

$\equiv\left[\right.$ equivalence $(j) \wedge E_{\text {in }}=\operatorname{set}\left(e 2\left(\right.\right.$ head $\left(P \_\right.$serial $\left.\left.)\right)\right)($original inputs $\left.)\right]$

$C V G \_B U I L D E R\left(P \_\right.$serial,$P \_$serial $)=$

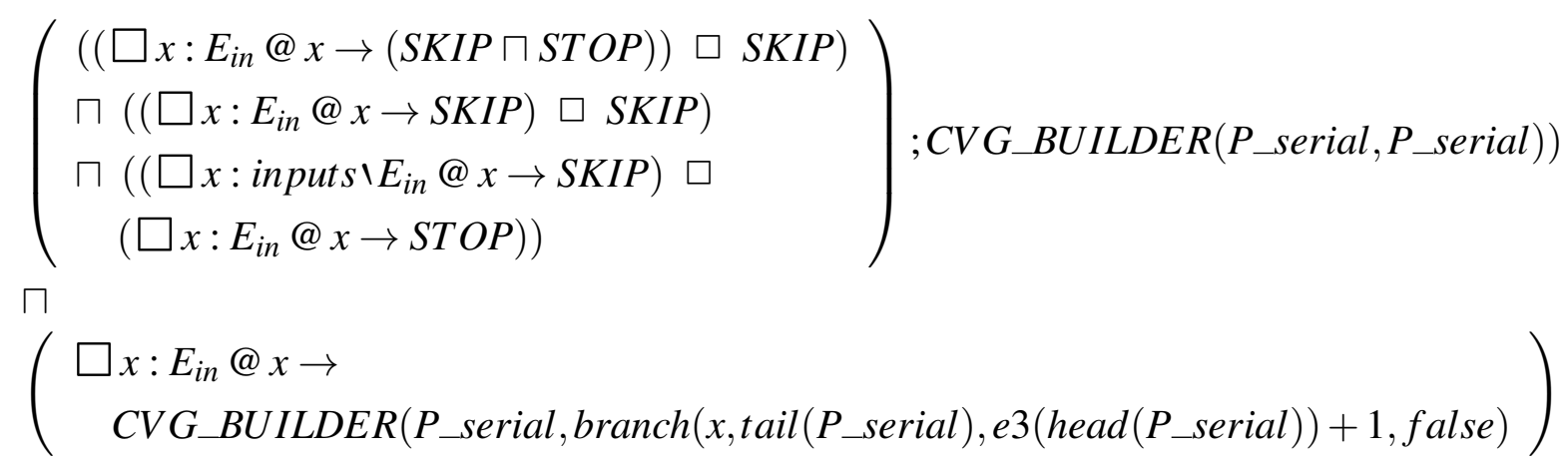

$\Rightarrow$ [failures semantics (Section 2.1.2) $\wedge \quad \square, \square$ semantics (ROSCOE, 1998)]

$\left(\langle\rangle, \Sigma \backslash\left(\right.\right.$ inputs $\left.\left.\backslash E_{\text {in }}\right)\right) \in \mathcal{F}\left(C V G \_B U I L D E R\left(P \_\right.\right.$serial,$P \_$serial $\left.)\right) \wedge$

$\left(\langle\rangle, \Sigma \backslash E_{\text {in }}\right) \in \mathcal{F}\left(C V G \_B U I L D E R\left(P \_\right.\right.$serial,$P \_$serial $\left.)\right)$

$\Rightarrow\left[P^{\prime} \operatorname{ecvg} P(\right.$ Definition 4.2$\left.)\right]$

$\left(\langle\rangle, \Sigma \backslash\left(\right.\right.$ inputs $\left.\left.\backslash E_{\text {in }}\right)\right) \in \mathcal{F}\left(C V G \_B U I L D E R\left(P \_\right.\right.$serial,$P \_$serial $\left.)\right) \wedge$

$\left(\langle\rangle, \Sigma \backslash E_{\text {in }}\right) \in \mathcal{F}\left(C V G \_B U I L D E R\left(P \_\right.\right.$serial,$P \_$serial $\left.)\right) \wedge$

$(\langle\rangle, X) \in \mathcal{F}\left(P^{\prime}\right) \mid X \subseteq \Sigma \Rightarrow$ 


$$
\begin{gathered}
\exists(\langle\rangle, Y) \in \mathcal{F}(P) \mid Y \cap \text { inputs } \supseteq X \cap \text { inputs } \wedge Y \cap \text { outputs } \subseteq X \cap \text { outputs } \\
\Rightarrow\left[\begin{array}{l}
E_{\text {in }}=\text { initials }(P) \subseteq \text { inputs } \wedge\left(\text { inputs } \backslash E_{\text {in }}\right) \cap \text { initials }(P)=\emptyset \wedge \\
G A P=1 \wedge \text { input determinism (Definition } 2.4) \wedge \text { set theory }
\end{array}\right] \\
\left\{\left(\langle\rangle, \Sigma \backslash\left(\text { inputs } \backslash E_{\text {in }}\right)\right),\left(\langle\rangle, \Sigma \backslash E_{\text {in }}\right)\right\} \subset \mathcal{F}\left(C V G \_B U I L D E R\left(P \_ \text {serial }, P \_ \text {serial }\right)\right) \wedge \\
\left(\langle\rangle, \Sigma \backslash E_{\text {in }}\right) \in \mathcal{F}(P) \wedge\left(\left(\langle\rangle, \Sigma \backslash\left(\text { inputs } \backslash E_{\text {in }}\right)\right) \in \mathcal{F}\left(P^{\prime}\right) \vee\left(\langle\rangle, \Sigma \backslash E_{\text {in }}\right) \in \mathcal{F}\left(P^{\prime}\right)\right) \\
\Rightarrow[\text { initials }(P) \nsubseteq \text { inputs } \wedge G A P=1] \text { (Second case) }
\end{gathered}
$$$$
\left\{\left(\langle\rangle, \Sigma \backslash\left(\text { inputs } \backslash E_{\text {in }}\right)\right),\left(\langle\rangle, \Sigma \backslash E_{\text {in }}\right)\right\} \subset \mathcal{F}\left(C V G \_B U I L D E R\left(P \_ \text {serial }, P \_ \text {serial }\right)\right) \wedge
$$$$
\left(\langle\rangle, \Sigma \backslash E_{\text {in }}\right) \in \mathcal{F}(P) \wedge\left(\left(\langle\rangle, \Sigma \backslash\left(\text { inputs } \backslash E_{\text {in }}\right)\right) \in \mathcal{F}\left(P^{\prime}\right) \vee\left(\langle\rangle, \Sigma \backslash E_{\text {in }}\right) \in \mathcal{F}\left(P^{\prime}\right)\right) \vee
$$

$C V G \_B U I L D E R\left(P \_\right.$serial,$P \_$serial $)=$

EXEC(inputs, 1$) ;\left(П x: \operatorname{set}\left(e 2\left(\right.\right.\right.$ head $\left(P \_\right.$serial $\left.\left.)\right)\right) @ x \rightarrow$

$C V G \_B U I L D E R\left(P \_\right.$serial, $\operatorname{branch}\left(x, \operatorname{tail}\left(P \_\right.\right.$serial $), e 3\left(\right.$ head $\left(P \_\right.$serial $\left.)\right)+1$, false $\left.\left.)\right)\right)$

$\equiv[$ equivalence $(b)]$

$\left\{\left(\langle\rangle, \Sigma \backslash\left(\right.\right.\right.$ inputs $\left.\left.\left.\backslash E_{\text {in }}\right)\right),\left(\langle\rangle, \Sigma \backslash E_{\text {in }}\right)\right\} \subset \mathcal{F}\left(C V G \_B U I L D E R\left(P \_\right.\right.$serial,$P \_$serial $\left.)\right) \wedge$

$\left(\langle\rangle, \Sigma \backslash E_{\text {in }}\right) \in \mathcal{F}(P) \wedge\left(\left(\langle\rangle, \Sigma \backslash\left(\right.\right.\right.$ inputs $\left.\left.\left.\backslash E_{\text {in }}\right)\right) \in \mathcal{F}\left(P^{\prime}\right) \vee\left(\langle\rangle, \Sigma \backslash E_{\text {in }}\right) \in \mathcal{F}\left(P^{\prime}\right)\right) \vee$

$C V G \_B U I L D E R\left(P \_\right.$serial,$P \_$serial $)=$

$((\sqcap x:$ inputs @ $(x \rightarrow S K I P)) \sqcap S K I P) ;\left(\sqcap x: \operatorname{set}\left(e 2\left(\right.\right.\right.$ head $\left(P \_\right.$serial $\left.\left.)\right)\right) @ x \rightarrow$ $C V G \_B U I L D E R\left(P \_\operatorname{serial}, \operatorname{branch}\left(x, \operatorname{tail}\left(P \_\right.\right.\right.$serial $), e 3\left(\right.$ head $\left(P \_\right.$serial $\left.\left.\left.\left.)\right)+1, f a l s e\right)\right)\right)$

$\Rightarrow$ [failures semantics (Section 2.1.2) $\wedge \quad \sqcap, \sqcap$ semantics (ROSCOE, 1998)]

$\left\{\left(\langle\rangle, \Sigma \backslash\left(\right.\right.\right.$ inputs $\left.\left.\left.\backslash E_{\text {in }}\right)\right),\left(\langle\rangle, \Sigma \backslash E_{\text {in }}\right)\right\} \subset \mathcal{F}\left(C V G \_B U I L D E R\left(P \_\right.\right.$serial,$P \_$serial $\left.)\right) \wedge$

$\left(\langle\rangle, \Sigma \backslash E_{\text {in }}\right) \in \mathcal{F}(P) \wedge\left(\left(\langle\rangle, \Sigma \backslash\left(\right.\right.\right.$ inputs $\left.\left.\left.\backslash E_{\text {in }}\right)\right) \in \mathcal{F}\left(P^{\prime}\right) \vee\left(\langle\rangle, \Sigma \backslash E_{\text {in }}\right) \in \mathcal{F}\left(P^{\prime}\right)\right) \vee$

$\{(\langle\rangle, \Sigma \backslash$ inputs $),(\langle\rangle, \Sigma \backslash e)\} \subset \mathcal{F}\left(C V G \_B U I L D E R\left(P \_\right.\right.$serial,$P \_$serial $\left.)\right) \mid e \in \operatorname{set}\left(e 2\left(\right.\right.$ head $\left(P \_\right.$serial $\left.\left.)\right)\right)$

$\Rightarrow\left[P^{\prime}\right.$ ecvg $P$ (Definition 4.2) $]$

$\left(\begin{array}{l}\left\{\left(\langle\rangle, \Sigma \backslash\left(\text { inputs } \backslash E_{\text {in }}\right)\right),\left(\langle\rangle, \Sigma \backslash E_{\text {in }}\right)\right\} \subset \mathcal{F}\left(C V G \_B U I L D E R\left(P \_ \text {serial }, P \_ \text {serial }\right)\right) \wedge \\ \left(\langle\rangle, \Sigma \backslash E_{\text {in }}\right) \in \mathcal{F}(P) \wedge\left(\left(\langle\rangle, \Sigma \backslash\left(\text { inputs } \backslash E_{\text {in }}\right)\right) \in \mathcal{F}\left(P^{\prime}\right) \vee\left(\langle\rangle, \Sigma \backslash E_{\text {in }}\right) \in \mathcal{F}\left(P^{\prime}\right)\right)\end{array}\right) \vee$ 


$$
\begin{aligned}
& \left(\begin{array}{c}
\{(\langle\rangle, \Sigma \backslash \text { inputs }),(\langle\rangle, \Sigma \backslash e)\} \subset \mathcal{F}\left(C V G \_B U I L D E R\left(P \_ \text {serial }, P \_ \text {serial }\right)\right) \mid \\
e \in \operatorname{set}\left(e 2\left(\text { head }\left(P \_ \text {serial }\right)\right)\right) \wedge \\
\exists(\langle\rangle, Y) \in \mathcal{F}(P) \mid Y \cap \text { inputs } \supseteq X \cap \text { inputs } \wedge Y \cap \text { out puts } \subseteq X \cap \text { outputs }
\end{array}\right) \\
& \Rightarrow\left[\begin{array}{l}
\left.\operatorname{set}\left(e 2\left(\text { head }\left(P \_ \text {serial }\right)\right)\right)\right)=\text { initials }(P) \subseteq \text { out puts } \wedge G A P=1 \\
\text { strong output decisiveness (Definition } 2.4) \wedge \text { set theory }
\end{array}\right] \\
& \left(\begin{array}{l}
\left\{\left(\langle\rangle, \Sigma \backslash\left(\text { inputs } \backslash E_{\text {in }}\right)\right),\left(\langle\rangle, \Sigma \backslash E_{\text {in }}\right)\right\} \subset \mathcal{F}\left(C V G \_B U I L D E R\left(P \_ \text {serial }, P \_ \text {serial }\right)\right) \wedge \\
\left(\langle\rangle, \Sigma \backslash E_{\text {in }}\right) \in \mathcal{F}(P) \wedge\left(\left(\langle\rangle, \Sigma \backslash\left(\text { inputs } \backslash E_{\text {in }}\right)\right) \in \mathcal{F}\left(P^{\prime}\right) \vee\left(\langle\rangle, \Sigma \backslash E_{\text {in }}\right) \in \mathcal{F}\left(P^{\prime}\right)\right)
\end{array}\right) \vee \\
& \left(\begin{array}{c}
\{(\langle\rangle, \Sigma \backslash \text { inputs }),(\langle\rangle, \Sigma \backslash e)\} \subset \mathcal{F}\left(C V G \_ \text {BUILDER }\left(P \_ \text {serial }, P \_ \text {serial }\right)\right) \mid \\
e \in \operatorname{set}(e 2(\text { head }(P \text { serial }))) \wedge \\
(\langle\rangle, \Sigma \backslash \text { inputs }) \in \mathcal{F}\left(P^{\prime}\right) \vee(\langle\rangle, \Sigma \backslash e) \text { in } \mathcal{F}\left(P^{\prime}\right) \mid e \in \operatorname{set}\left(e 2\left(\text { head }\left(P \_ \text {serial }\right)\right)\right)
\end{array}\right) \\
& \Rightarrow\left[\begin{array}{l}
\text { induction over } C V G \_B U I L D E R\left(P \_ \text {serial }, P \_ \text {serial }\right) \\
\text { structure until } e 1(\text { head }(\text { crt }))==\text { end }(\text { Listing } 6)
\end{array}\right] \\
& \forall(t, X) \in \mathcal{F}\left(P^{\prime}\right) \Rightarrow(t, X) \in \mathcal{F}\left(C V G \_B U I L D E R\left(P \_ \text {serial }, P \_ \text {serial }\right)\right) \mid G A P=1 \\
& \equiv[\text { failures semantics }]
\end{aligned}
$$

$C V G \_B U I L D E R\left(P \_\right.$serial,$P \_$serial $) \sqsubseteq_{\mathrm{F}} P^{\prime} \mid G A P=1$

\section{[Third part]}

$\left[G A P=n(n \geq 2) \wedge C V G \_B U I L D E R\left(P \_\right.\right.$serial,$P \_$serial $)($Listing 6) $\wedge e 1$ definition $($ Listing 4$)]$

$e 1\left(\right.$ head $\left(P \_\right.$serial $\left.)\right) \neq$end

$\Rightarrow[\operatorname{initials}(P) \subseteq$ inputs $] \quad$ (First case)

$C V G \_B U I L D E R\left(P \_\right.$serial,$P \_$serial $)=$

EXEC_Q $\left(\right.$ inputs, set $\left(e 2\left(\right.\right.$ head $\left(P \_\right.$serial $\left.\left.\left.)\right)\right), n\right) ; C V G \_B U I L D E R\left(P \_s e r i a l, P \_\right.$serial $\left.)\right)$

$\sqcap \quad\left(\square x: \operatorname{set}\left(e 2\left(\right.\right.\right.$ head $\left.\left.\left(P \_s e r i a l\right)\right)\right) @ x \rightarrow$

$C V G \_B U I L D E R\left(P \_\operatorname{serial}, \operatorname{branch}\left(x, \operatorname{tail}\left(P \_\right.\right.\right.$serial $), e 3\left(\right.$ head $\left(P \_\right.$serial $\left.)\right)+1$, false $\left.)\right)$

$\sqsubseteq_{F}\left[\right.$ refinements $(k)$ and $(l) \wedge E_{\text {in }}=\operatorname{set}\left(e 2\left(\right.\right.$ head $\left(P \_\right.$serial $\left.\left.)\right)\right)($original inputs $\left.)\right]$

$C V G \_B U I L D E R\left(P \_\right.$serial,$P \_$serial $)=$

$\left(\begin{array}{l}E X E C \_Q\left(\text { inputs }, E_{\text {in }}, 1\right) ; \\ E X E C_{-} Q\left(\text { inputs }, E_{\text {in }}, n-1\right)\end{array}\right) ; C V G \_B U I L D E R\left(P \_\right.$serial,$P \_$serial $\left.)\right)$

$\sqcap$

$\left(\begin{array}{l}\square x: E_{\text {in }} @ x \rightarrow \\ C V G \_B U I L D E R\left(P \_\operatorname{serial}, \operatorname{branch}\left(x, \operatorname{tail}\left(P \_ \text {serial }\right), e 3\left(\text { head }\left(P \_ \text {serial }\right)\right)+1, \text { false }\right)\right.\end{array}\right)$ 
$\Rightarrow$ [failures semantics (Section 2.1.2) $\wedge \quad \square, \square$ and; semantics (ROSCOE, 1998)]

$\left(\begin{array}{l}\left\{\left(\langle\rangle, \Sigma \backslash\left(\text { inputs } \backslash E_{\text {in }}\right)\right),\left(\langle\rangle, \Sigma \backslash E_{\text {in }}\right),\left(t, \Sigma \backslash\left(\text { inputs } \backslash E_{\text {in }}\right)\right)\right\} \subset \\ \mathcal{F}\left(C V G \_B U I L D E R\left(P \_ \text {serial }, P \_ \text {serial }\right)\right) \mid\left(t \in\left(\text { input } \backslash \backslash E_{\text {in }}\right)^{*} \wedge \# t<n\right)\end{array}\right)$

$\Rightarrow\left[P^{\prime}\right.$ ecvg $P$ (Definition 4.2$\left.)\right]$

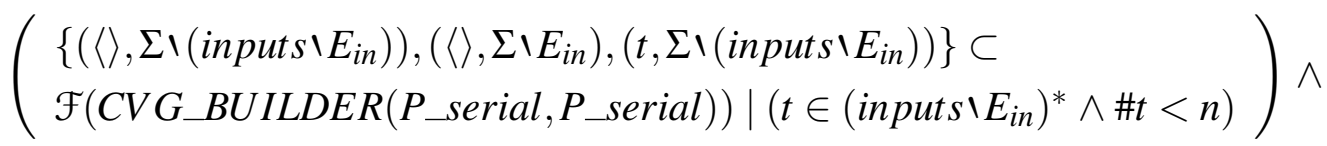

$(\langle\rangle, X) \in \mathcal{F}\left(P^{\prime}\right) \mid X \subseteq \Sigma \Rightarrow$

$\exists(\langle\rangle, Y) \in \mathcal{F}(P) \mid Y \cap$ inputs $\supseteq X \cap$ inputs $\wedge Y \cap$ outputs $\subseteq X \cap$ outputs

$\Rightarrow\left[\begin{array}{l}E_{\text {in }}=\text { initials }(P) \subseteq \text { inputs } \wedge\left(\text { inputs } \backslash E_{\text {in }}\right) \cap \text { initial } s(P)=\emptyset \wedge \\ G A P=n \wedge \text { input determinism }(\text { Definition } 2.4) \wedge \text { set theory }\end{array}\right]$

$\left(\begin{array}{l}\left\{\left(\langle\rangle, \Sigma \backslash\left(\text { inputs } \backslash E_{\text {in }}\right)\right),\left(\langle\rangle, \Sigma \backslash E_{\text {in }}\right),\left(t, \Sigma \backslash\left(\text { inputs } \backslash E_{\text {in }}\right)\right)\right\} \subset \\ \mathcal{F}\left(C V G \_ \text {BUILDER }\left(P \_ \text {serial }, P \_ \text {serial }\right)\right) \mid\left(t \in\left(\text { inputs } \backslash E_{\text {in }}\right)^{*} \wedge \# t<n\right)\end{array}\right) \wedge$

$\left(\langle\rangle, \Sigma \backslash E_{\text {in }}\right) \in \mathcal{F}(P) \wedge\left(\begin{array}{l}\left(\langle\rangle, \Sigma \backslash\left(\text { inputs } \backslash E_{\text {in }}\right)\right) \in \mathcal{F}\left(P^{\prime}\right) \vee\left(\langle\rangle, \Sigma \backslash E_{\text {in }}\right) \in \mathcal{F}\left(P^{\prime}\right) \vee \\ \left(t, \Sigma \backslash\left(\text { inputs } \backslash E_{\text {in }}\right)\right) \in \mathcal{F}\left(P^{\prime}\right) \mid\left(t \in\left(\text { input } \backslash E_{\text {in }}\right)^{*} \wedge \# t<n\right)\end{array}\right)$

$\Rightarrow[$ initials $(P) \nsubseteq$ inputs $\wedge G A P=n(n \geq 2) \wedge$ equivalences $(c)$ and $(d)]$

$\left(\begin{array}{l}\left(\begin{array}{l}\left\{\left(\langle\rangle, \Sigma \backslash\left(\text { inputs } \backslash E_{\text {in }}\right)\right),\left(\langle\rangle, \Sigma \backslash E_{\text {in }}\right),\left(t, \Sigma \backslash\left(\text { inputs } \backslash E_{\text {in }}\right)\right)\right\} \subset \\ \mathcal{F}\left(C V G \_ \text {BUILDER }\left(P_{\text {_serial }}, P_{\_} \text {serial }\right)\right) \mid\left(t \in\left(\text { inputs } \backslash E_{\text {in }}\right)^{*} \wedge \# t<n\right)\end{array}\right) \wedge \\ \left(\langle\rangle, \Sigma \backslash E_{\text {in }}\right) \in \mathcal{F}(P) \wedge\left(\begin{array}{l}\left(\langle\rangle, \Sigma \backslash\left(\text { inputs } \backslash E_{\text {in }}\right)\right) \in \mathcal{F}\left(P^{\prime}\right) \vee\left(\langle\rangle, \Sigma \backslash E_{\text {in }}\right) \in \mathcal{F}\left(P^{\prime}\right) \vee \\ \left(t, \Sigma \backslash\left(\text { input } \backslash \backslash E_{\text {in }}\right)\right) \in \mathcal{F}\left(P^{\prime}\right) \mid\left(t \in\left(\text { inputs } \backslash E_{\text {in }}\right)^{*} \wedge \# t<n\right)\end{array}\right)\end{array}\right) \vee$

$C V G \_B U I L D E R\left(P \_\right.$serial,$P \_$serial $)=$

EXEC(inputs, 1$) ; E X E C($ inputs,$n-1) ;\left(П x: \operatorname{set}\left(e 2\left(\right.\right.\right.$ head $\left(P \_\right.$serial $\left.\left.)\right)\right) @ x \rightarrow$ $C V G \_B U I L D E R\left(P \_\operatorname{serial}, \operatorname{branch}\left(x, \operatorname{tail}\left(P \_\right.\right.\right.$serial $), e 3\left(\right.$ head $\left(P \_\right.$serial $\left.)\right)+1$, false $\left.\left.)\right)\right)$

$\Rightarrow$ [failures semantics (Section 2.1.2) $\wedge \quad \square$ and ; semantics (ROSCOE, 1998)]

$\left\{\left(\langle\rangle, \Sigma \backslash\left(\right.\right.\right.$ inputs $\left.\left.\left.\backslash E_{\text {in }}\right)\right),\left(\langle\rangle, \Sigma \backslash E_{\text {in }}\right)\right\} \subset \mathcal{F}\left(C V G \_B U I L D E R\left(P \_\right.\right.$serial,$P \_$serial $\left.)\right) \wedge$ $\left(\begin{array}{c}\left(\begin{array}{c}\left\{\left(\langle\rangle, \Sigma \backslash\left(\text { inputs } \backslash E_{\text {in }}\right)\right),\left(\langle\rangle, \Sigma \backslash E_{\text {in }}\right),\left(t, \Sigma \backslash\left(\text { inputs } \backslash E_{\text {in }}\right)\right)\right\} \subset \\ \mathcal{F}\left(C V G \_B U I L D E R\left(P \_ \text {serial }, P \_ \text {serial }\right)\right) \mid\left(t \in\left(\text { inputs } \backslash E_{\text {in }}\right)^{*} \wedge \# t<n\right)\end{array}\right) \wedge \\ \left(\langle\rangle, \Sigma \backslash E_{\text {in }}\right) \in \mathcal{F}(P) \wedge\left(\begin{array}{l}\left(\langle\rangle, \Sigma \backslash\left(\text { inputs } \backslash E_{\text {in }}\right)\right) \in \mathcal{F}\left(P^{\prime}\right) \vee\left(\langle\rangle, \Sigma \backslash E_{\text {in }}\right) \in \mathcal{F}\left(P^{\prime}\right) \vee \\ \left(t, \Sigma \backslash\left(\text { inputs } \backslash E_{\text {in }}\right)\right) \in \mathcal{F}\left(P^{\prime}\right) \mid\left(t \in\left(\text { input } \backslash E_{\text {in }}\right)^{*} \wedge \# t<n\right)\end{array}\right)\end{array}\right) \vee$
$\left(\begin{array}{c}\{(\langle\rangle, \Sigma \backslash \text { inputs }),(\langle\rangle, \Sigma \backslash e),(t, \Sigma \backslash e)\} \subset \mathcal{F}\left(C V G \_B U I L D E R\left(P \_ \text {serial }, P \_ \text {serial }\right)\right) \\ \mid e \in \text { set }\left(e 2\left(\text { head }\left(P \_ \text {serial }\right)\right)\right) \wedge\left(t \in\left(\text { inputs } \backslash E_{\text {in }}\right)^{*} \wedge \# t<n\right)\end{array}\right)$ 
$\Rightarrow\left[P^{\prime}\right.$ ecvg $P($ Definition 4.2$\left.)\right]$

$\left\{\left(\langle\rangle, \Sigma \backslash\left(\right.\right.\right.$ inputs $\left.\left.\left.\backslash E_{\text {in }}\right)\right),\left(\langle\rangle, \Sigma \backslash E_{\text {in }}\right)\right\} \subset \mathcal{F}\left(C V G \_B U I L D E R\left(P \_\right.\right.$serial,$P \_$serial $\left.)\right) \wedge$

$\left(\begin{array}{c}\left(\begin{array}{l}\left\{\left(\langle\rangle, \Sigma \backslash\left(\text { inputs } \backslash E_{\text {in }}\right)\right),\left(\langle\rangle, \Sigma \backslash E_{\text {in }}\right),\left(t, \Sigma \backslash\left(\text { input } \backslash \backslash E_{\text {in }}\right)\right)\right\} \subset \\ \mathcal{F}\left(C V G \_B U I L D E R\left(P \_ \text {serial }, P \_ \text {serial }\right)\right) \mid\left(t \in\left(\text { inputs } \backslash E_{\text {in }}\right)^{*} \wedge \# t<n\right)\end{array}\right) \wedge \\ \left(\langle\rangle, \Sigma \backslash E_{\text {in }}\right) \in \mathcal{F}(P) \wedge\left(\begin{array}{l}\left(\langle\rangle, \Sigma \backslash\left(\text { inputs } \backslash E_{\text {in }}\right)\right) \in \mathcal{F}\left(P^{\prime}\right) \vee\left(\langle\rangle, \Sigma \backslash E_{\text {in }}\right) \in \mathcal{F}\left(P^{\prime}\right) \vee \\ \left(t, \Sigma \backslash\left(\text { input } \backslash E_{\text {in }}\right)\right) \in \mathcal{F}\left(P^{\prime}\right) \mid\left(t \in\left(\text { inputs } \backslash E_{\text {in }}\right)^{*} \wedge \# t<n\right)\end{array}\right)\end{array}\right) \vee$

$\left(\begin{array}{c}\{(\langle\rangle, \Sigma \backslash \text { inputs }),(\langle\rangle, \Sigma \backslash e),(t, \Sigma \backslash e)\} \subset \mathcal{F}\left(C V G \_B U I L D E R\left(P \_ \text {serial }, P \_ \text {serial }\right)\right) \\ \mid e \in \operatorname{set}\left(e 2\left(\text { head }\left(P \_ \text {serial }\right)\right)\right) \wedge\left(t \in\left(\text { input } \backslash \backslash E_{\text {in }}\right)^{*} \wedge \# t<n\right) \\ \wedge \exists(\langle\rangle, Y) \in \mathcal{F}(P) \mid Y \cap \text { inputs } \supseteq X \cap \text { inputs } \wedge Y \cap \text { out puts } \subseteq X \cap \text { outputs }\end{array}\right)$

$\Rightarrow\left[\begin{array}{l}\left.\operatorname{set}\left(e 2\left(\text { head }\left(P \_ \text {serial }\right)\right)\right)\right)=\text { initials }(P) \subseteq \text { out puts } \wedge G A P=n(n \geq 2) \\ \text { strong output decisiveness (Definition } 2.4) \wedge \text { set theory }\end{array}\right]$

(Second case)

$\left\{\left(\langle\rangle, \Sigma \backslash\left(\right.\right.\right.$ inputs $\left.\left.\left.\backslash E_{\text {in }}\right)\right),\left(\langle\rangle, \Sigma \backslash E_{\text {in }}\right)\right\} \subset \mathcal{F}\left(C V G \_B U I L D E R\left(P \_\right.\right.$serial,$P \_$serial $\left.)\right) \wedge$

$\left(\begin{array}{l}\left(\begin{array}{l}\left\{\left(\langle\rangle, \Sigma \backslash\left(\text { inputs } \backslash E_{\text {in }}\right)\right),\left(\langle\rangle, \Sigma \backslash E_{\text {in }}\right),\left(t, \Sigma \backslash\left(\text { input } \backslash E_{\text {in }}\right)\right)\right\} \subset \\ \mathcal{F}\left(C V G \_ \text {BUILDER }\left(P \_ \text {serial }, P \_ \text {serial }\right)\right) \mid\left(t \in\left(\text { inputs } \backslash E_{\text {in }}\right)^{*} \wedge \# t<n\right)\end{array}\right) \wedge \\ \left(\langle\rangle, \Sigma \backslash E_{\text {in }}\right) \in \mathcal{F}(P) \wedge\left(\begin{array}{l}\left(\langle\rangle, \Sigma \backslash\left(\text { input } \backslash E_{\text {in }}\right)\right) \in \mathcal{F}\left(P^{\prime}\right) \vee\left(\langle\rangle, \Sigma \backslash E_{\text {in }}\right) \in \mathcal{F}\left(P^{\prime}\right) \vee \\ \left(t, \Sigma \backslash\left(\text { inputs } \backslash E_{\text {in }}\right)\right) \in \mathcal{F}\left(P^{\prime}\right) \mid\left(t \in\left(\text { input } \backslash E_{\text {in }}\right)^{*} \wedge \# t<n\right)\end{array}\right)\end{array}\right) \vee$

$\left(\begin{array}{c}\{(\langle\rangle, \Sigma \backslash \text { inputs }),(\langle\rangle, \Sigma \backslash e),(t, \Sigma \backslash e)\} \subset \mathcal{F}\left(C V G \_B U I L D E R\left(P \_ \text {serial }, P \_ \text {serial }\right)\right) \\ \wedge\left((\langle\rangle, \Sigma \backslash \text { inputs }) \in \mathcal{F}\left(P^{\prime}\right) \vee(\langle\rangle, \Sigma \backslash e),(t, \Sigma \backslash e) \in \mathcal{F}\left(P^{\prime}\right)\right) \\ \mid e \in \operatorname{set}\left(e 2\left(\text { head }\left(P \_ \text {serial }\right)\right)\right) \wedge\left(t \in\left(\text { input } \backslash \backslash E_{\text {in }}\right)^{*} \wedge \# t<n\right)\end{array}\right)$

$\Rightarrow\left[\begin{array}{l}\text { induction over } C V G \_B U I L D E R\left(P \_ \text {serial }, P \_ \text {serial }\right) \\ \text { structure until } e 1(\text { head }(\text { crt }))==\text { end }(\text { Listing } 6)\end{array}\right]$

$\forall(t, X) \in \mathcal{F}\left(P^{\prime}\right) \Rightarrow(t, X) \mathcal{F}\left(C V G \_B U I L D E R\left(P \_\right.\right.$serial,$P \_$serial $\left.)\right) \mid G A P=n(n \geq 2)$

$\equiv[$ failures semantics $] \quad C V G \_B U I L D E R\left(P \_\right.$serial,$P \_$serial $) \sqsubseteq_{\mathrm{F}} P^{\prime} \mid G A P=n(n \geq 2)$

The construction of Tester_ecvg follows the same principles used to build Tester_cvg. The differences relate to the helper processes, mainly because they must be able to do, after a new-in-context input, a sequence of any new-in-context events, before converging (Definition 4.4). Processes $E X E C \_D$ and $E X E C \_Q$ remain as presented before.

As we need to detect an occurrence of a new-in-context input before allowing any new-incontext event we did a subtle change in the EXEC process: it acknowledges (by communicating the in_ack event) every time some event happens, making it possible to know when a new-incontext input was communicated.

Process $A F T \_I N$ acts like a watcher for in_acks events, reverberating them by commu- 
nicating the in_rdt event. Process EXEC_AFTER_IN is able to catch the in_rdt event and turn back to the $E X E C$ 's execution. Note that playing the role of watchers, both process cannot force anything to happen, so the successful termination, $S K I P$, is always a possibility.

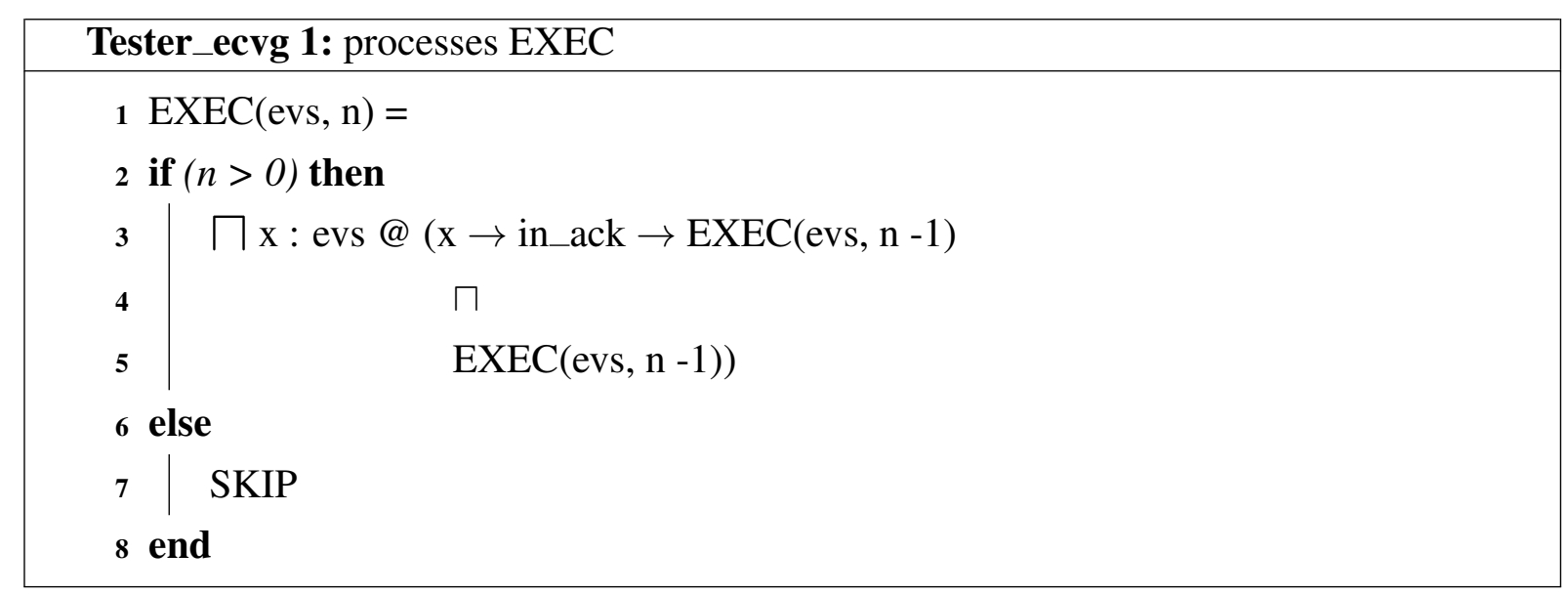

Tester_ecvg 2: processes AFT_IN and EXEC_AFTER_IN

AFT_IN $=$ in_ack $\rightarrow$ in_rdt $\rightarrow$ SKIP $\square$ SKIP

EXEC_AFTER_IN $($ evs,n $)=$ in $\_$rdt $\rightarrow$ EXEC(evs, n) $\square$ SKIP

Process $E X E C_{-} Q \_A F T$ acts like a wrapper to $E X E C_{-} Q$. It is parametrised by three sets of events evs 1 , evs 2 and evs 3 : the first two have the same intent of their counterparts in $E X E C \_Q$, where the latter was conceived to receive the set containing the events that can happen after a new-in-context input of evs1 levs2 (line 1). After an input, EXEC_Q synchronises on in_ack with $A F T \_I N$ (line 2), which in turn communicates $i n \_r d t$, at this time synchronising with $E X E C \_A F T E R \_I N$, which is responsible for offering up to $n$ times any evs 3 levs 2 (inputs or outputs) new-in-context events (line 4).

Process $E X E C \_A F T$ follows the same reasoning used in $E X E C \_Q \_A F T$, but it uses $E X E C$ instead of $E X E C \_Q$ : it is designed to be used before an internal choice among outputs, therefore there is no need to retain the external choice of expected inputs, as $E X E C_{-} Q$ does.

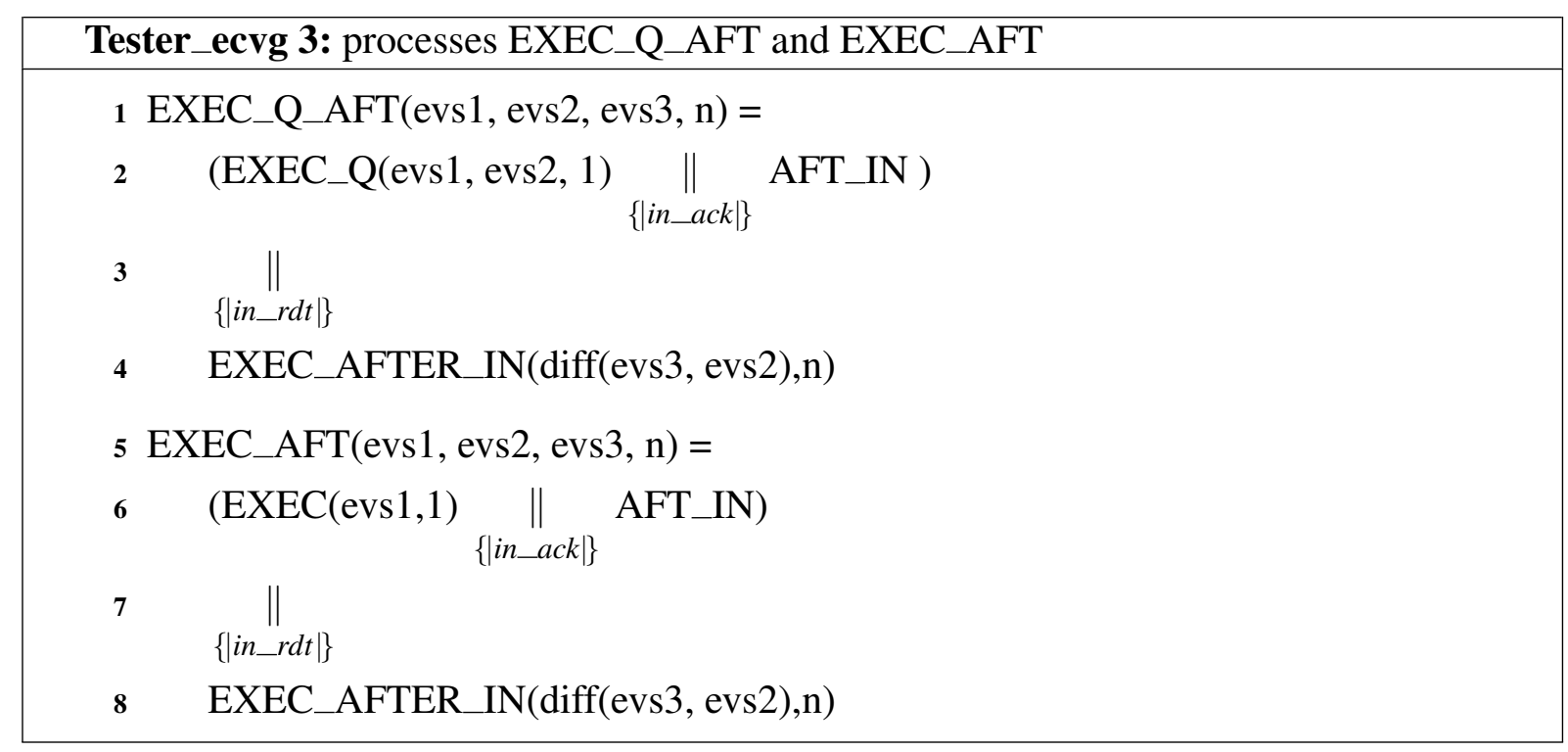


Process $E C V G \_B U I L D E R$ coordinates the helper processes we have seen in the work of building Tester_ecvg $(P)$ for an I/O processes $P$, based on its serialised representation src (line 1). It distinguishes from $C V G \_B U I L D E R$ by the use of the helper process $E X E C \_Q \_A F T$ and EXEC_AFT instead of EXEC_Q and EXEC, respectively. The process EXEC_Q $\_A F T$ (line 6) can execute up to $n$ (where $n=G A P$ ) new-in-context events (all=inputs $\cup$ outputs), but the first must be an input; as mentioned earlier, it differs from EXEC_AFT (line 12) as it preserves the external choice among expected inputs.

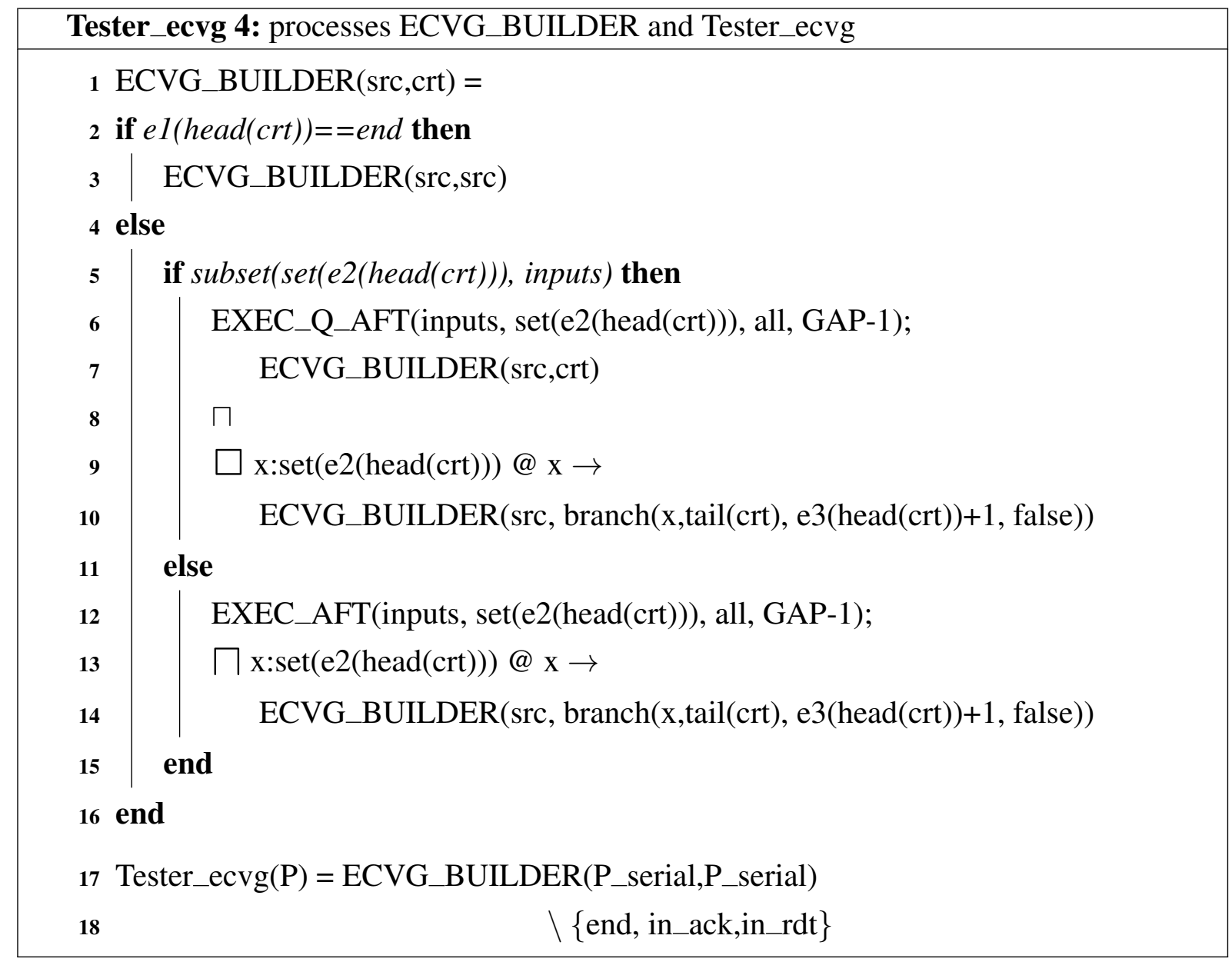

Now if we consider the CSP processes $P$ and $P^{\prime}$ in Figure 4.3, the following FDR assertions hold. As before the second assertion holds as a consequence of Lemma 4.1, from where we know $P$ io_ecvg $P$ because $P \sqsubseteq_{\mathrm{F}} P$.

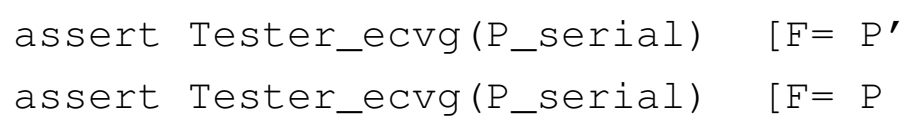




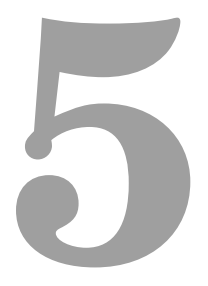

\section{Case Studies}

In this chapter we develop three case studies to show the applicability of our $\mathcal{B} \mathcal{R J C}$ refinement and inheritance relations. We show how to evolve, in a stepwise approach, componentbased specifications by the use of refinement to encompass implementation details, and by inheritance, which adds new functionalities or enhances the existing ones in a way complementary to refinement.

\section{Bank system}

In this case study we model a bank system, improved by the use of inheritance. The ATM process interacts with customers (CUS process) to provide services like withdraw, deposit, transfer and check balance, available after successful login. The ATM process responds to CUS requests non-deterministically, since it has no ability to communicate with a server. Therefore we developed the ATM2 process, that has the ability to handle user requests to a bank server, in our case study the BankServer process. All definitions bellow use $\operatorname{CSP}_{M}$, a CSP machine readable dialect, as introduced in Chapter 2.

We start by defining some data types: each account has a number (ACC_NUM), a pin (ACC_PIN), a balance (MONEY) and an associated card (CARD), which can be blocked. Success (SUCC_TYPES) and error (ERR_TYPES) values are also modelled.

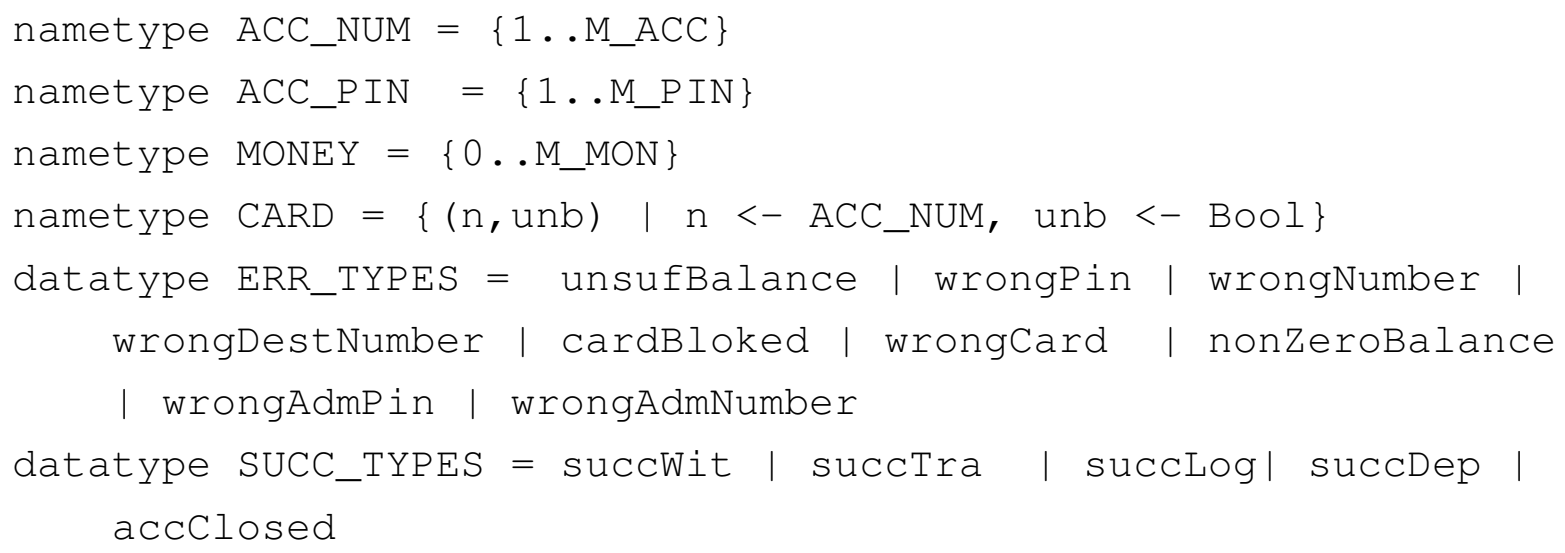


Input events for ATM are outputs for CUS and vice versa (ATM_IN and ATM_OUT stand for inputs and outputs of ATM). The ATM process communicates through channel atm_cus, whose type is I_DTATM_CUS. This tags ATM_IN and ATM_OUT with in and out labels, respectively, so we can distinguish inputs from outputs. The CUS process communicates though channel cus, whose type is I_DTCUS. Note that it labels values inversely to I_DTATM_CUS.

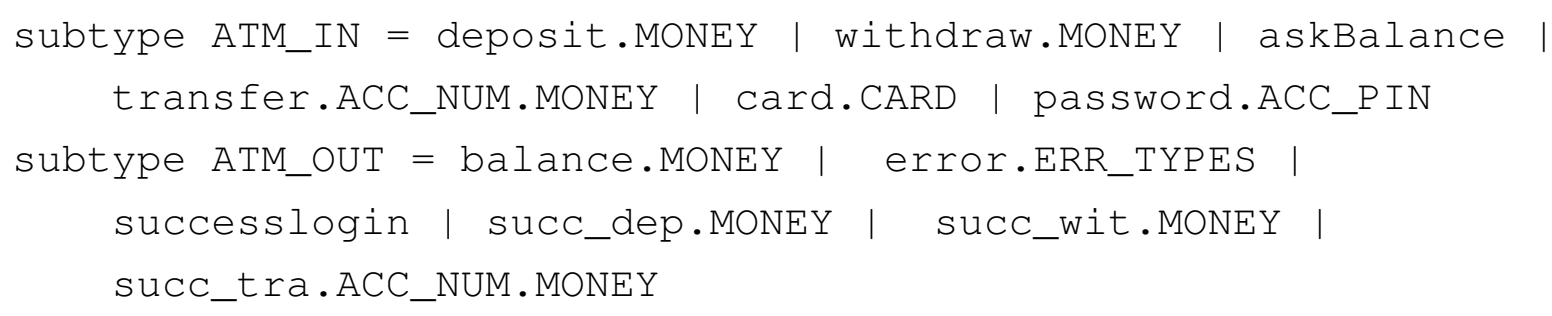

When a card is inserted in the ATM machine, the machine can ask for the account's password or throw an error if the card is blocked atm_cus.out.error!cardBloked. After receiving the password, it decides internally if either the user has entered a wrong pin (atm_cus.out.error.wrongPin), the card is unblocked but not recognised (atm_ cus.out.error!wron gCard) or the user has entered a correct pin (atm_cus.out.suc cesslogin). In the last case, the user may proceed by selecting one among deposit, withdraw, transfer and balance check operations. Note the non-determinism exhibited by the ATM when informing the balance ( $|\sim| \mathrm{Vb}:$ MONEY). It happens because ATM has no means to decide on a particular value, i.e., it is not connected to any bank server.

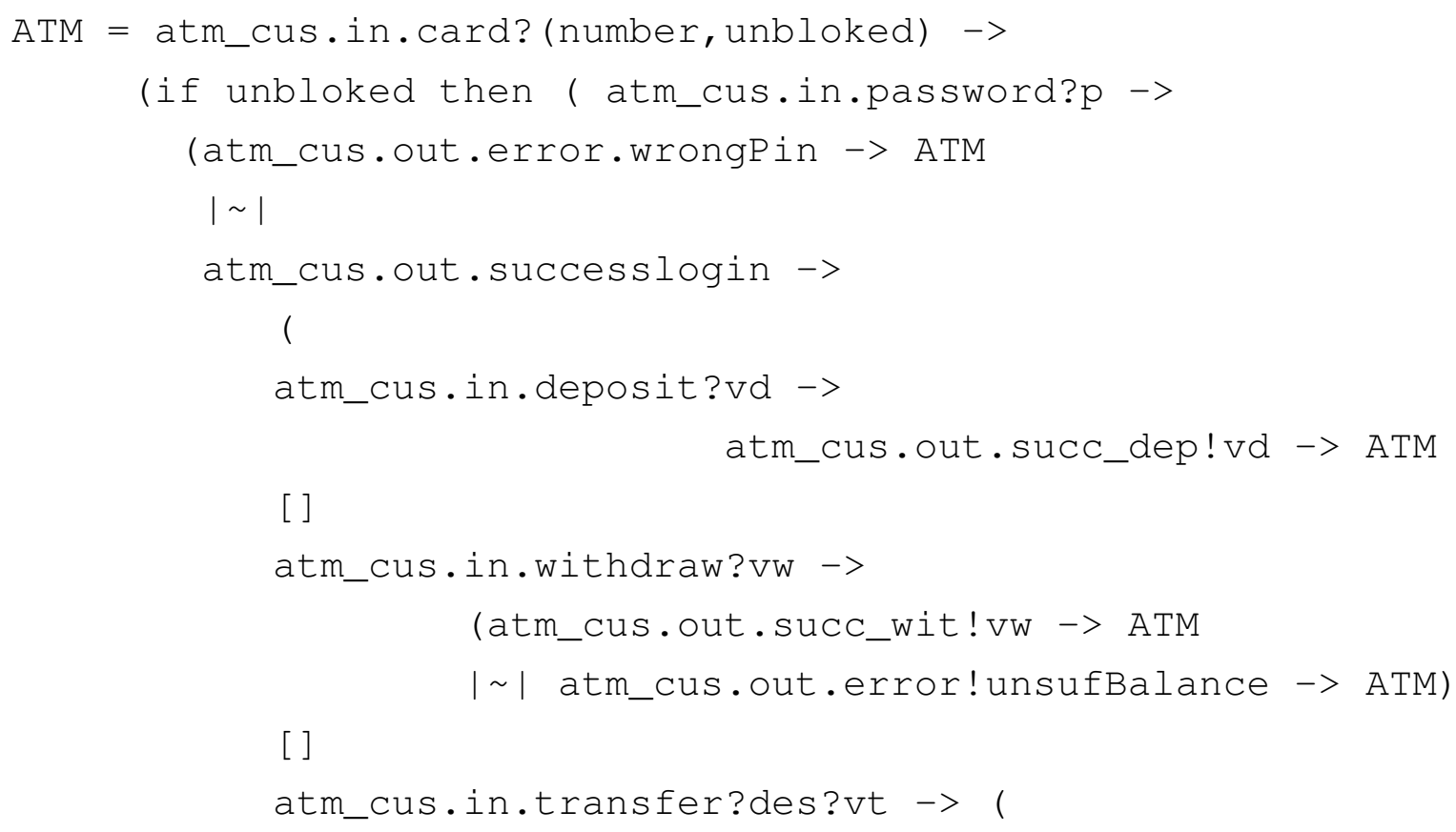




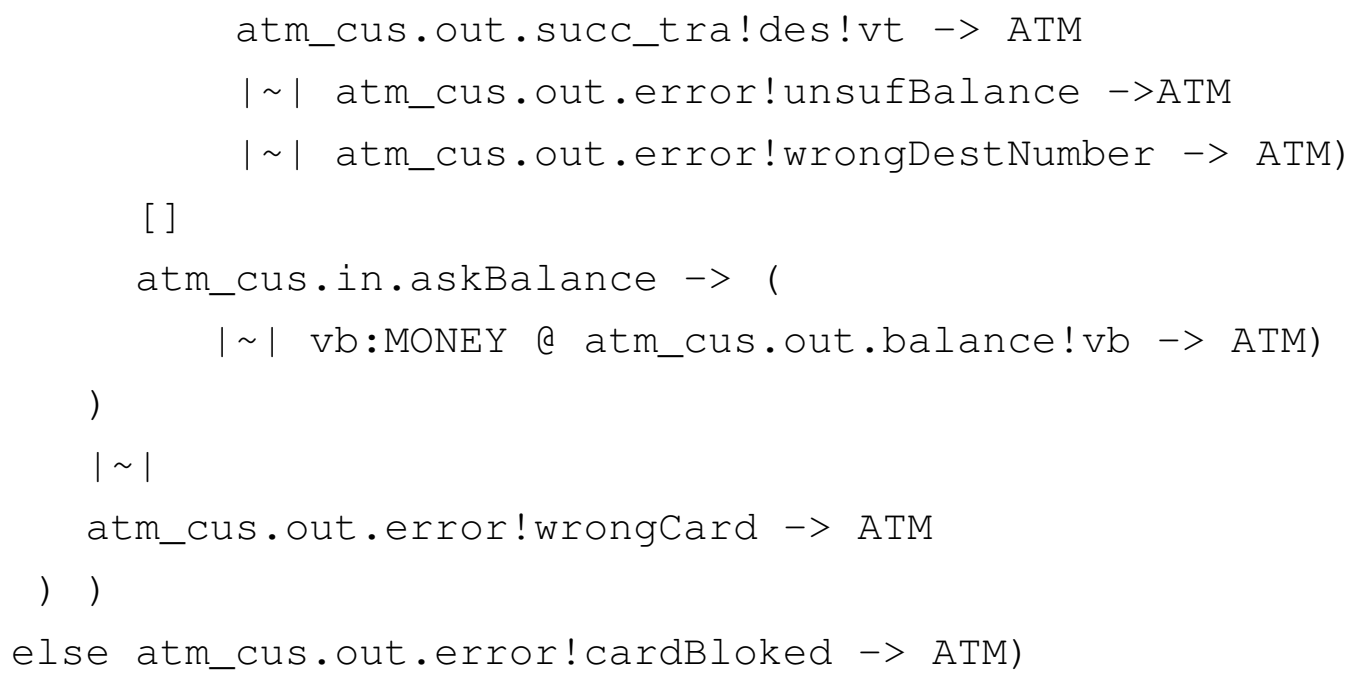

Initially, the customer CUS process communicates with ATM inserting his card. If unblocked, the user provides a password cus . out. pas sword! p. If correct (cus. in. succes slogin) it can proceed by choosing between deposit (cus. out.deposit. vd), withdraw (cus.out.withdraw.vw), transfer (cus.out. transfer!des!vt) or balance check (cus.out.askBalance).

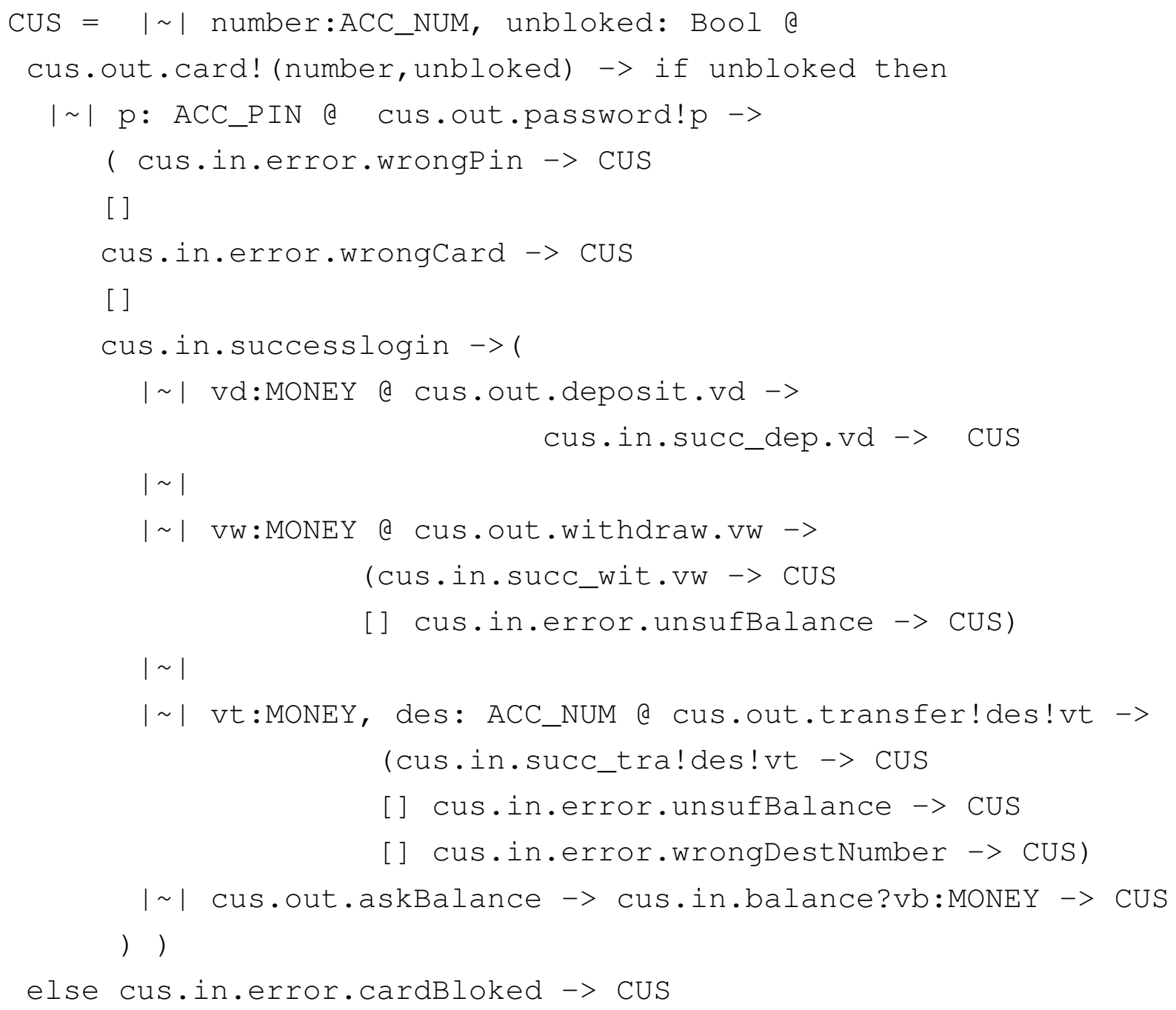


Figure 5.1: $\operatorname{Ctr}_{S Y S}=C t r_{A T M}\left[\mathrm{~atm} \_\right.$cus $\leftrightarrow$ cus $] C t r_{C U S}$

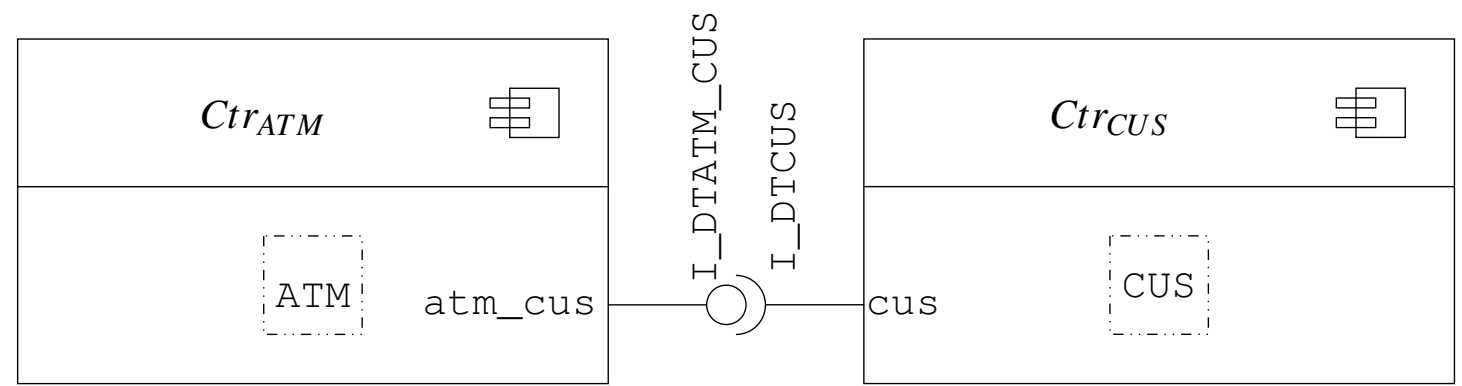

Source: José Dihego

In $\mathcal{B R J C}$, the processes ATM and CUS are encapsulated as the behaviour of the component contracts $C_{t} r_{A T M}$ and $\operatorname{Ctr}_{C U S}$.

$$
\begin{aligned}
& C t r_{A T M} \widehat{=}\left\langle\begin{array}{l}
\text { ATM, }\{\text { atm_cus } \mapsto \text { I_DTATM_CUS } \\
\{\text { I_DTATM_CUS }\},\{\text { atm_cus }\}
\end{array}\right\}, \\
& C t r_{C U S} \widehat{=}\langle\text { CUS, }\{\text { cus } \mapsto \text { I_DTCUS }\},\{\text { I_DTCUS }\},\{\text { cus }\}\rangle
\end{aligned}
$$

These can be connected by the channels atm_cus and cus generating Ctr $_{S Y S}$ (Figure 5.1), which, since it has been built from basic deadlock-free components and using the $\mathcal{B R J C}$ communication composition rule (COMM), is also deadlock free as we can check using FDR3.

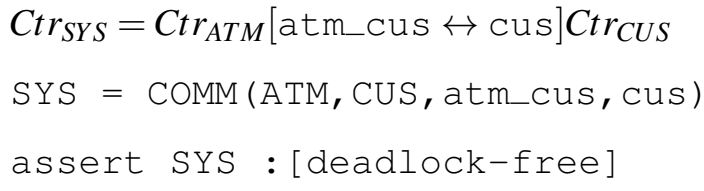

In this system, we can guarantee that the communications between ATMs and customers do not deadlock, but actually ATM does not provide reliable services to CUS, since, for example, the costumer might be charged an amount that is different from that dispensed in a withdraw. It happens because ATM does not have the ability to connect to any kind of server. We address such concerns by creating the process BankServer and by improving the ATM behaviour, as ATM2 process, which is able to communicate with the process BankServer. As we did before, we create two subsets of data types: SER_IN and SER_OUT. They stand, respectively, for the BankServer's inputs and outputs. Interface I_DTSER just tags these sets with in and out labels. Inputs for the BankServer will be outputs for ATM2, and vice-versa, so the interface I_DTATM tags values inversely to I_DTSER. The channels bk and atm_bk will be used, respectively, by BankServer and ATM2 to establish a communication medium between them. 


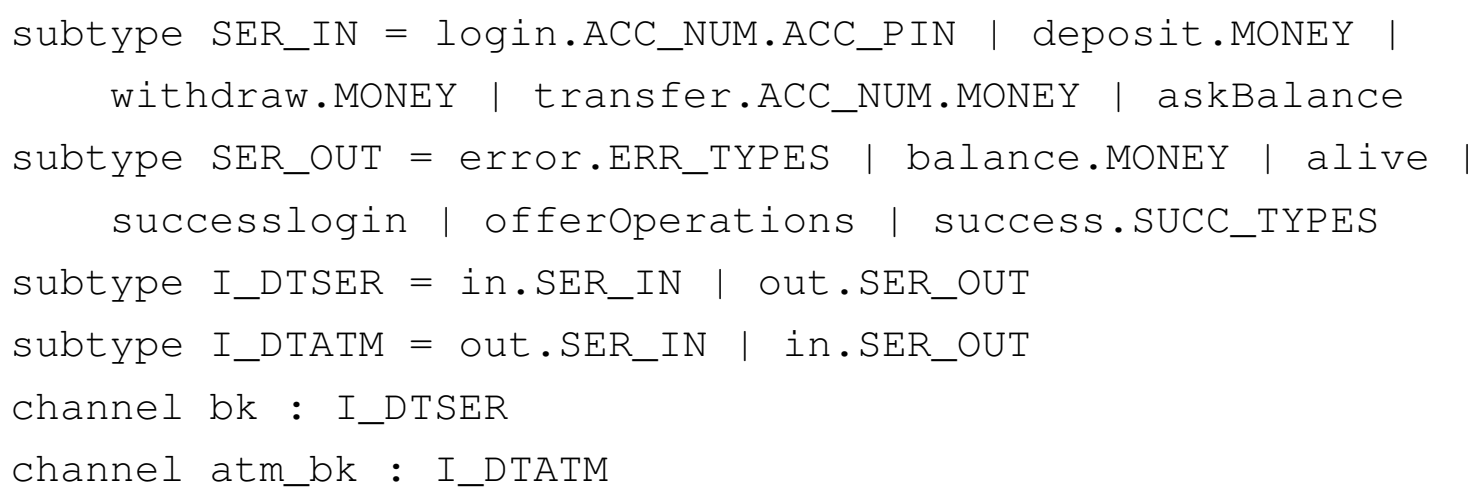

BankServer is parameterised by a set of accounts. When it is ready to interact (bk. out.alive), the server receives the user's credentials, (bk.in.login? num?pin) and, if either the account number is unknown (bk . out.error!wrongNumber) or a wrong pin (bk . out . error! wrongPin) is provided, the process goes back to its initial state, without any change. Otherwise, it acknowledges a successful login (bk . out. succe ss . succLog) and offers (bk . out . offeroperations) the aforementioned bank's operations.

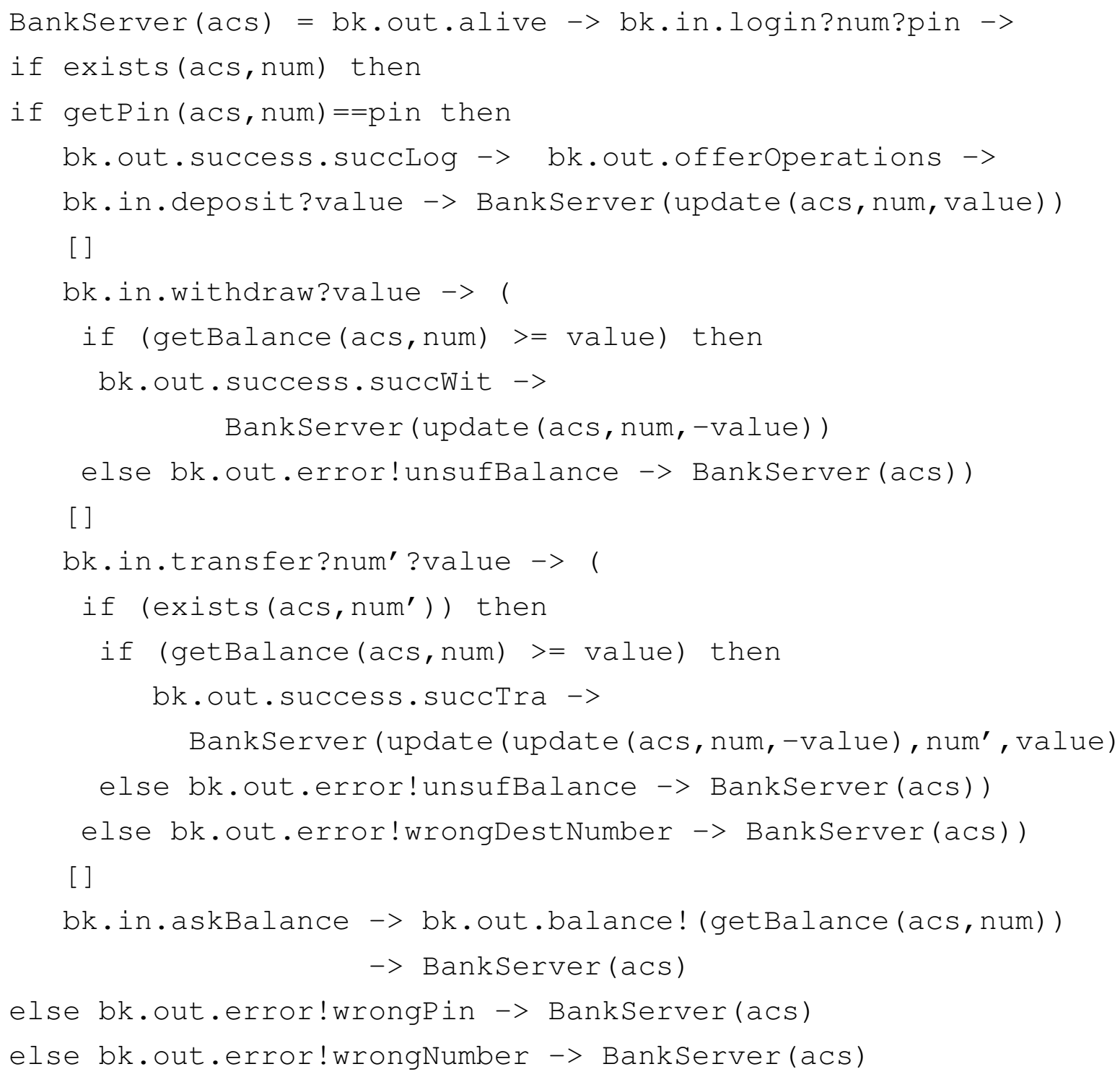


Process ATM2, from the CUS's perspective, continues to offer the same services offered by ATM, but now it has the ability to communicate with the BankServer process and deliver more deterministic and sound services. As well as ATM, ATM2 receives the user's card atm_cus.in.card, then waits for for his password. Nevertheless, instead of taking a nondeterministic decision about the correctness of login credentials, the process ATM2 waits for a server's alive acknowledge (atm_bk. in . a live), sends the user's credentials (atm_bk . out . $\log i n !$ number!p) to the server, and waits a server response informing either that the pin is wrong (atm_bk.in.error.wrongPin) or there is no account with this number (atm_bk. in.error.wrongNumber) or the user credentials are correct (atm_bk.in. success. succLog).

Whatever ATM2 receives form the BankServer it retransmits to CUS. This pattern is repeated for operations that follow a successful login.

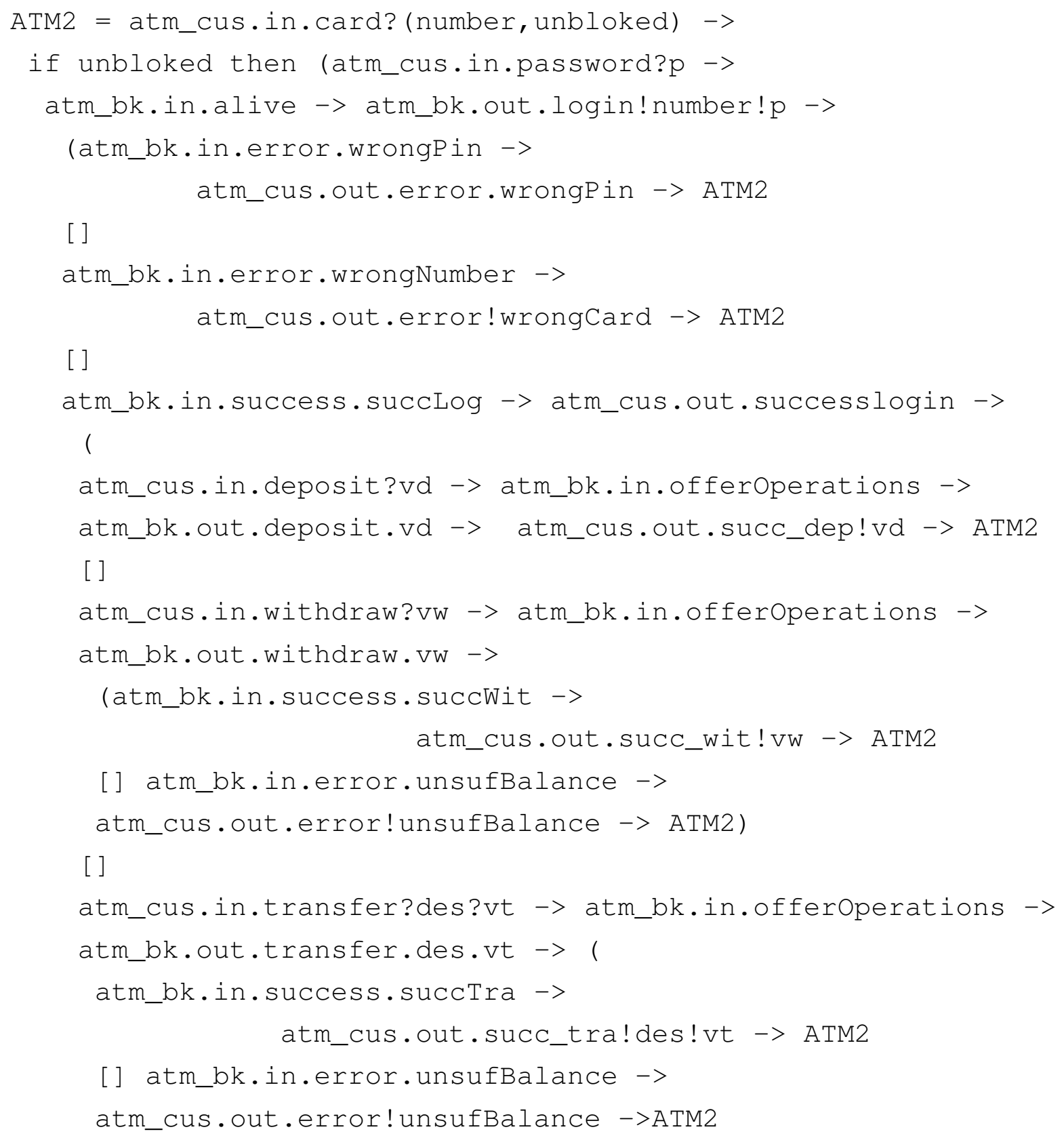




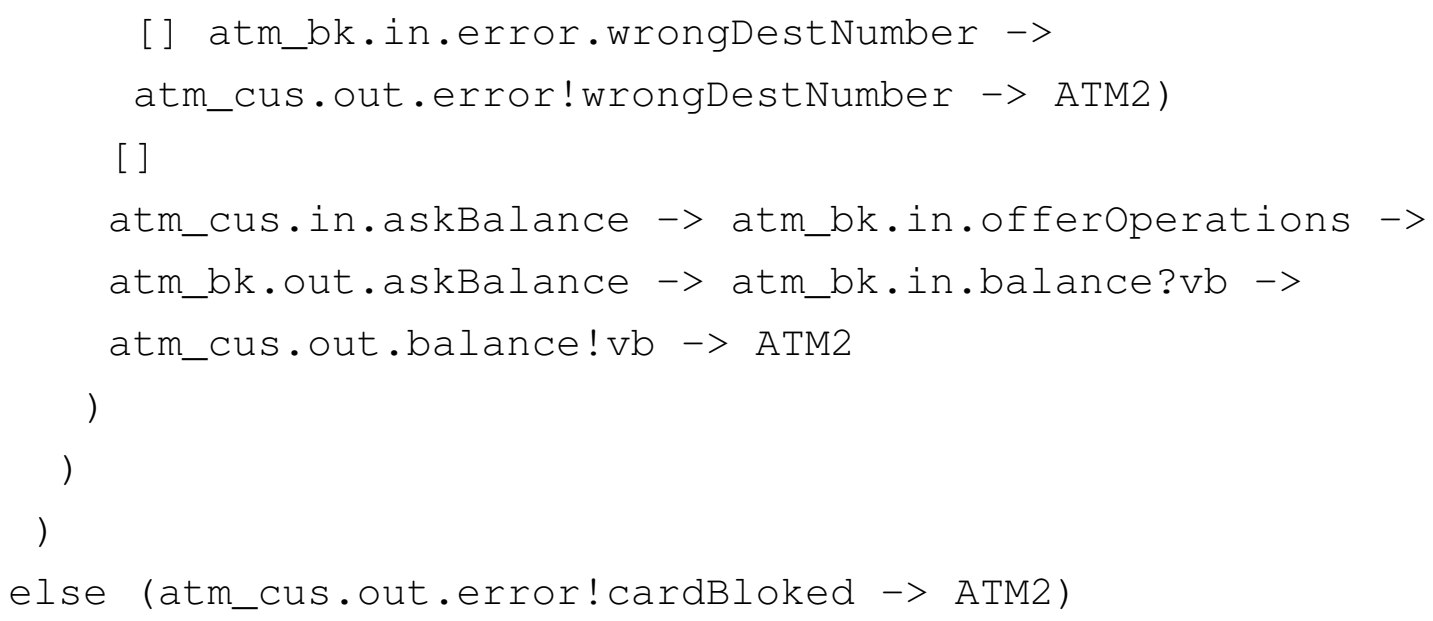

In the $\mathcal{B R J C}$ model, the processes ATM2 and BankServer (acs) are represented, respectively, by:

$$
\begin{aligned}
& C t r_{A T M 2} \widehat{=} \\
& \left\langle\begin{array}{l}
\text { ATM2, }\{\text { atm_cus } \mapsto \text { I_DTATM_CUS, atm_bk } \mapsto \text { I_DTATM } \\
\{\text { I_DTATM_CUS, I_DTATM }\},\{\text { atm_cus, atm_bk }\}
\end{array}\right\rangle \\
& \operatorname{Ctr}_{\text {BankServer(acs) }} \widehat{=} \\
& \left\langle\begin{array}{l}
\text { BankServer (accounts) },\{\mathrm{bk} \mapsto \text { I_DTSER }\} \\
\{\text { I_DTSER }\},\{\mathrm{bk}\}
\end{array}\right\rangle
\end{aligned}
$$

The most important result of the presented model is that, by Definition 4.7, we have that $C t r_{A T M} \triangleleft e_{e v g} C t r_{A T M 2}$ and by Theorem 4.2, the component contract $C t r_{S Y S 2}$, which is obtained from $C_{t r} r_{S Y 1}$ by replacing $C t r_{A T M}$ with $C t r_{A T M 2}$ (Figure 5.2) is deadlock free. In Appendix A (Section A.1), we present the automated verification (as an assertion in FDR3) which ensures that convergence holds between the I/O processes ATM and ATM2. This exemplifies that it is always possible replace a component by another that inherits from it, preserving the property of deadlock-freedom (Theorem 4.2).

$$
C t r_{S Y S 2}=C t r_{A T M 2}[\text { atm_cus } \leftrightarrow \text { cus }] C t r_{C U S}
$$

We complete our design by connecting $C t r_{\text {BankServer (acs) }}$ and $C t r_{S Y S 2}$ via their channels, bk and atm_bk, respectively (Figure 5.3). The resulting component is deadlock free by construction.

$$
\operatorname{Ctr}_{S Y S 2}\left[\mathrm{~atm} \_\mathrm{bk} \leftrightarrow \mathrm{bk}\right] \operatorname{Ctr}_{\text {BankServer(acs })}
$$


Figure 5.2: $C t r_{S Y S 2}=C \operatorname{tr}_{A T M 2}[\mathrm{~atm} \operatorname{cus} \leftrightarrow \mathrm{cus}] C t r_{C U S}$

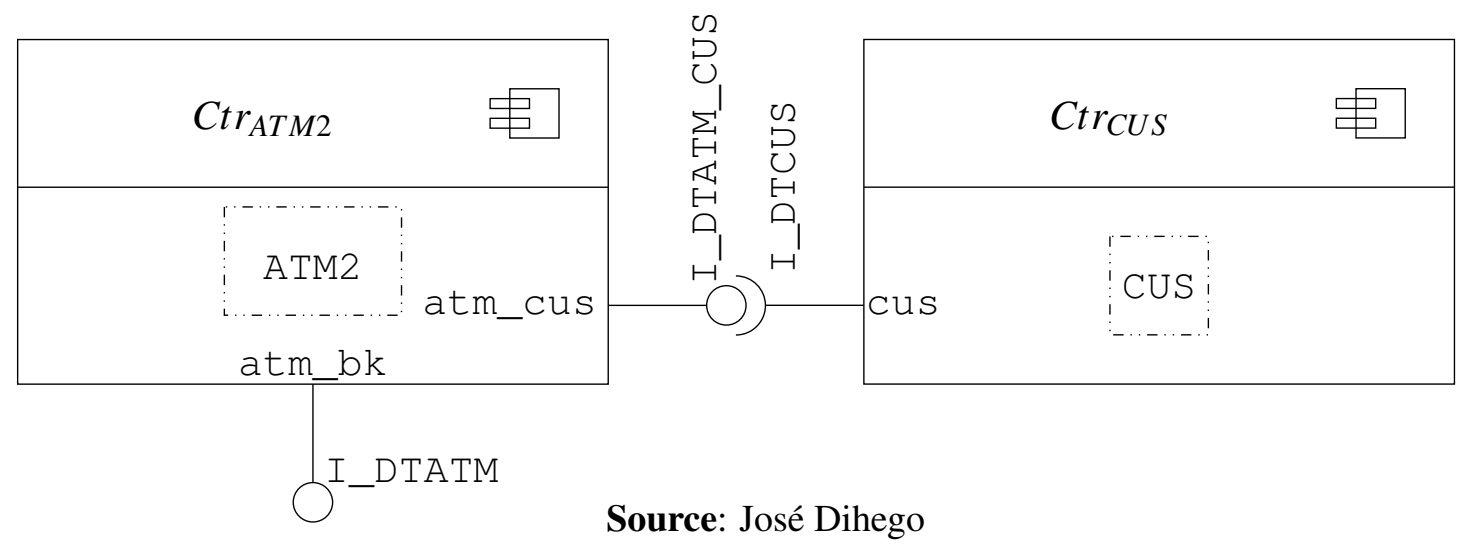

Figure 5.3: $C t r_{S Y S 2}\left[\mathrm{~atm} \_\mathrm{bk} \leftrightarrow \mathrm{bk}\right] \operatorname{Ctr}_{\text {BankServer(acs) }}$

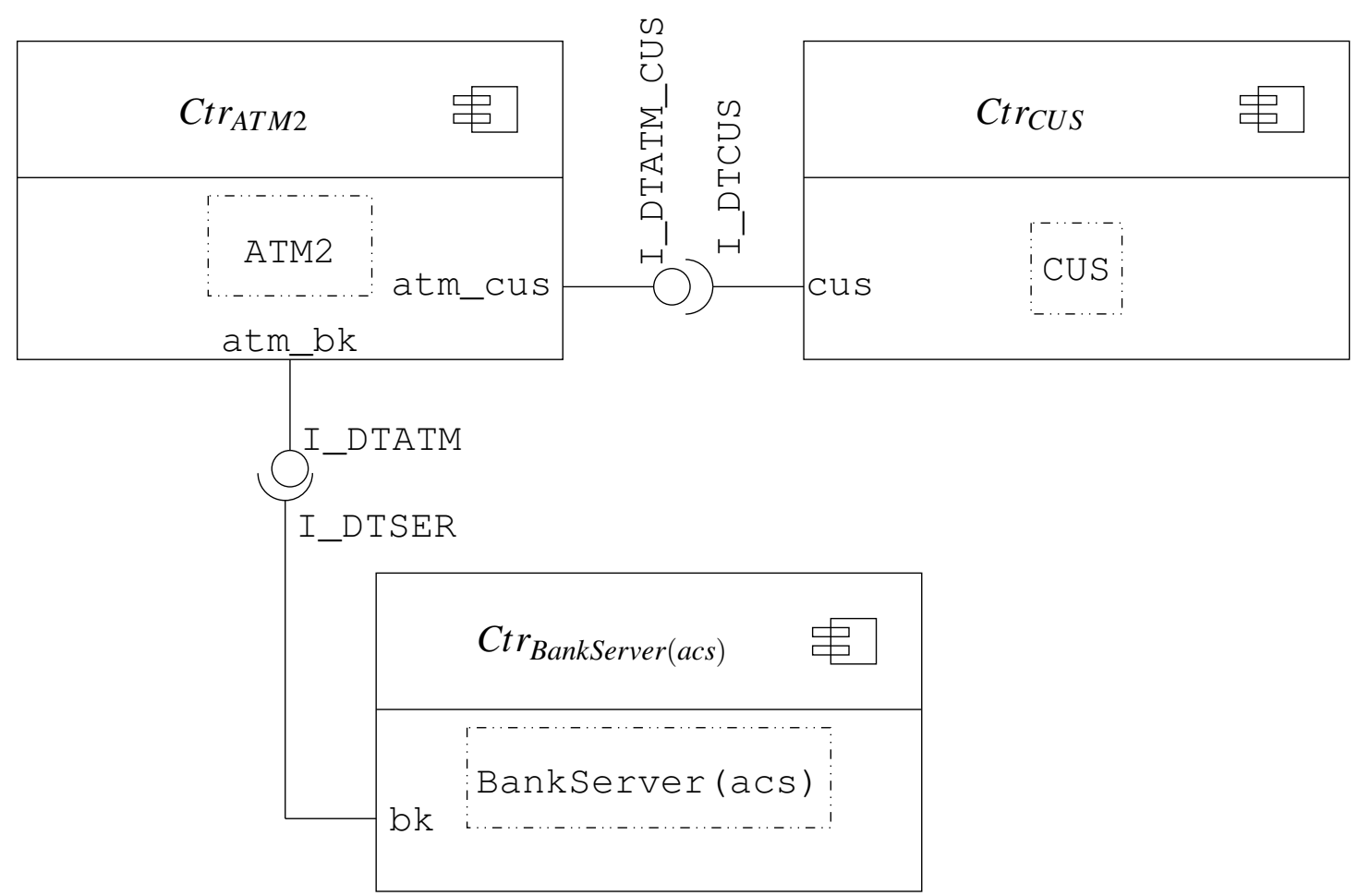

Source: José Dihego 


\section{An autonomous healthcare robot}

We model an autonomous healthcare robot that monitors and medicates patients, being able to contact the relevant individuals or systems in case of emergency. It receives data from a number of sensors and actuates by injecting intravenous drugs and/or by calling the emergency medical services and the patient's relatives or neighbours. We use the following data types: BI (breath intensity), BT (body temperature), DD (drug dose), BGL (blood glucose level), CL (call list, the relevant individuals to be called in the case of emergency), DRUG (the drugs in the robot's actuators), QUEST (the robot's question list, to ask the patient when its voice recognition module is used).

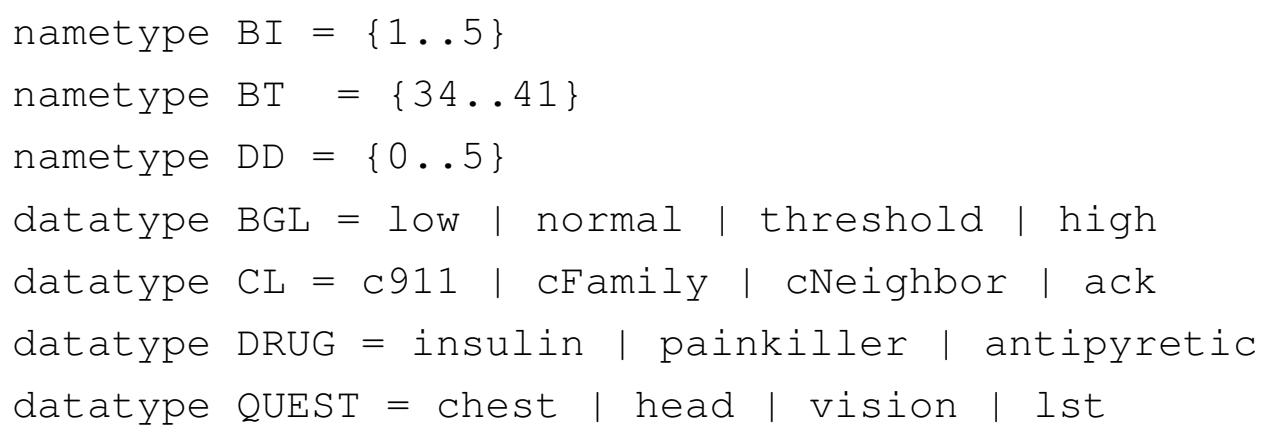

These types are composed into more elaborated ones whose data will be communicated through the channels used to connect sensors, actuators and phones to the robot. The set EVENTS encompasses the data sent though in and out of: the body attached sensors (breath.BI bodyTemp.BT and bloodGlucose.BGL), the vision recognition devices (numbnessface.Bool and fainting.Bool), the noise recognition device (cough.Bool and trouble Speaking.Bool), the voice interaction devices (visionTrouble.Bool, chestDiscomfort.Bool, headache. Bool and ask. QUEST), the phone interface (call .CL) and the intravenous injection actuator (administer.DRUG.DD).

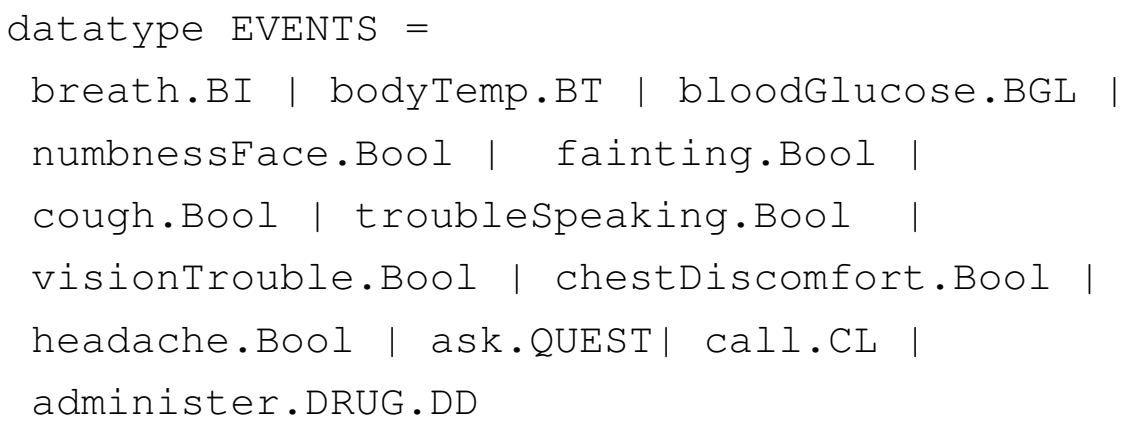

An event in EVENTS can be communicated as an output to a component and become an input to the other to which it connects by one of the BRJC composition rules (Section 2.3.3). Therefore we define IO as the set EVENTS where each value is tagged with in and out, which differentiates inputs from outputs.

datatype IO = out.EVENTS | in.EVENTS 
Each sensor/device communicates with the robot component via a specific channel, according to this schema: bodySen, the body attached sensors; imageRec, the vision recognition devices/sensors; voiceRec, the noise recognition devices/sensors; talk - the voice interaction devices/sensors; phone, the phone's interface and intravenousNeedle, the intravenous injection actuator. Each channel has its own type (a subset of IO) that involves only functionality related events. As an example, consider a channel with the I_BS type (I_BS $\subset$ IO and BS $\subset$ EVENTS), then it can communicate any event registered by the body attached sensors: breath, body temperature and blood glucose level.

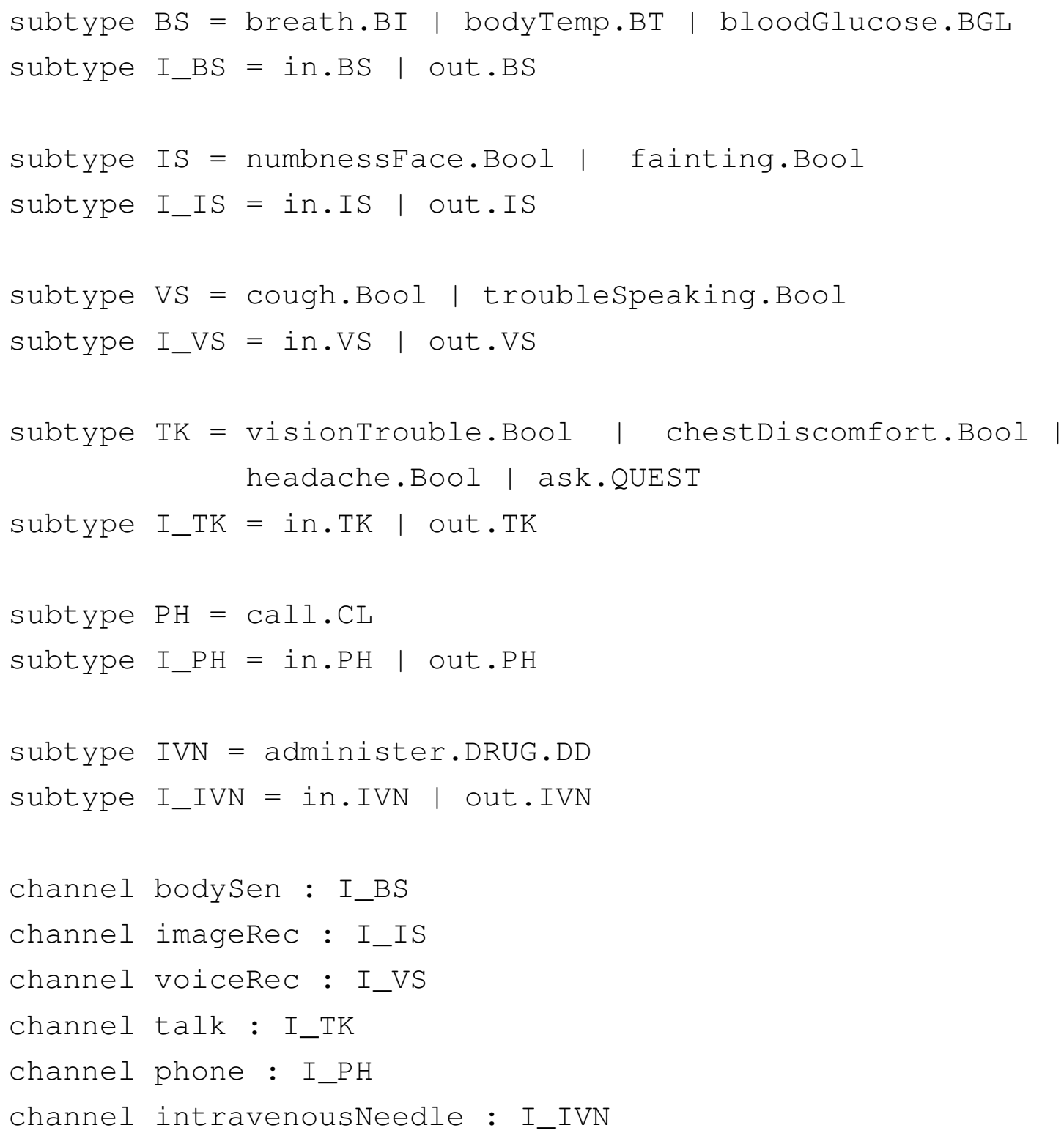

The behaviour of our healthcare robot is defined in terms of the I/O process HC_BOT. It waits for the breath level indicator; if this level is critical $(<3)$, then it behaves as MOD_CALL_P 1 (module phone call priority one), which contacts a patient's neighbour, the registered emergency service and relatives, in that order; then it waits for at least two of them to acknowledge before coming back to its initial state. Otherwise, the patient is breathing normally, and the 
robot reads the noise sensor to check whether she is coughing (voiceRec.in.cough?b). If so, it reads the body temperature (bodysen.in.bodyTemp?t) and blood glucose sensors (bodySen. in.bloodGlucose?g). If the body temperature exceeds 38 degrees Celsius, then it administers a dose of antipyretic (intravenousNeedle. out.administer. anti pyretic.d_ap). If the blood glucose level is in the threshold or high, it administers insulin (intravenousNeedle.out.administer.in-sulin.d_in), otherwise it just comes back to its initial state. After administrating any drug, and before coming back to its initial state, the robot must contact the patient's neighbour and relatives by behaving as MOD_CALL_P2 (module phone call priority two), in which case at least one of them must acknowledge.

If the patient is breathing normally but in silence, the robot asks the image recognition module to inform about: any unusual sign in his face (imageRec. in. numbnessFace?nf) or if he fainted (imageRec.in.fainting?f). If at least one condition holds, the robot administers a painkiller (intravenousNeedle. out. administer.painkiller.d_pk), calls the relevant individuals by behaving as MOD_CALL_P 1 . In any case, it goes to its initial state.

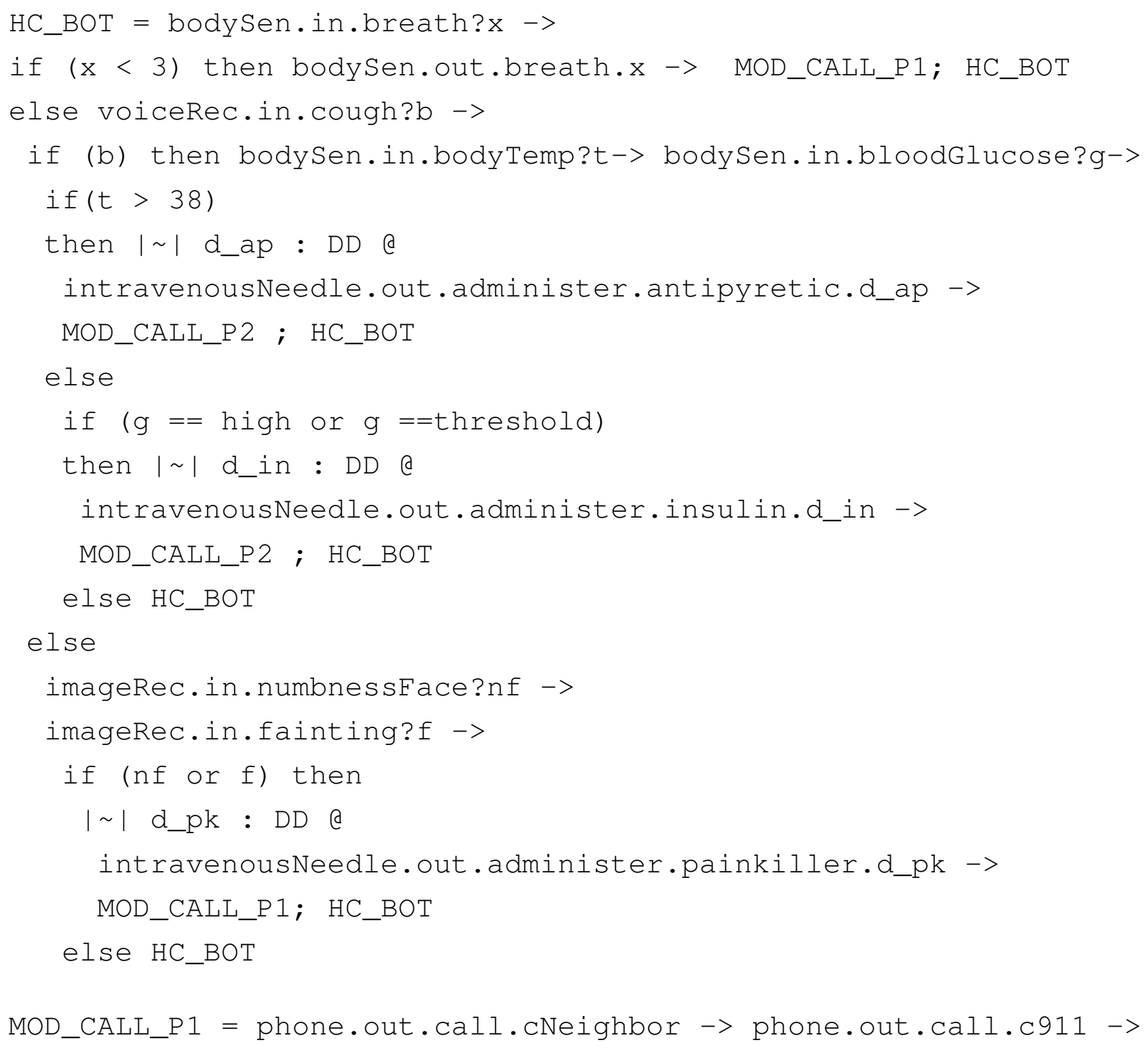


phone.out.call.cFamily $\rightarrow$ phone.in.call.ack ->

phone.in.call.ack $\rightarrow$ SKIP

MOD_CALL_P2 = phone.out.call.cNeighbor ->

phone.out.call.cFamily $\rightarrow$ phone.in.call.ack $\rightarrow$ SKIP

MOD_CALL_P3 = phone.out.call.cFamily ->

phone.in.call.ack $\rightarrow$ SKIP

In $\mathcal{B R J C}$, the healthcare robot is defined in terms of the $\mathrm{Ctr}_{H C \_B O T}$ contract. It behaves as HC_BOT and can interact with its environment by one of its visible communication channels: bodySen, imageRec, voiceRec, phone and intravenousNeedle.

$$
\begin{aligned}
& C t r_{H C \_B O T} \widehat{=}
\end{aligned}
$$

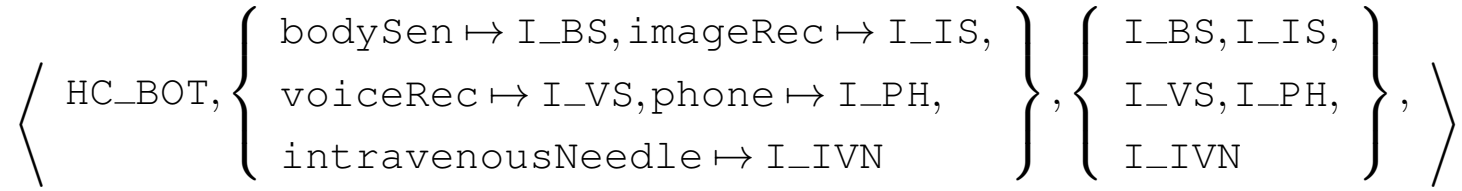

$$
\begin{aligned}
& \text { \{bodySen, imageRec, voiceRec, phone, intravenousNeedle\} }
\end{aligned}
$$

The robot $C t r_{H C \_B O T}$ is able to diagnose and select the appropriate drug to be administered. Nevertheless, there is no criteria to define an appropriate drug dose given the seriousness of the patient condition; in fact it is a non deterministic decision. For example, consider: | | ds : DD \& intravenousNeedle.out.administer.antipyretic.ds; no matter the seriousness of the fever, we cannot know what will be the dose ds to be administered to the patient. The I/O process HC_BOT_ACC addresses this question by using two criteria: each degree above 38 degrees Celsius will correspond to a unite of antipyretic (intravenousNeedle.out.administer.antipyretic.to37) and, the insulin dose will be one or two unities if the blood glucose level is on the threshold or greater than two, otherwise.

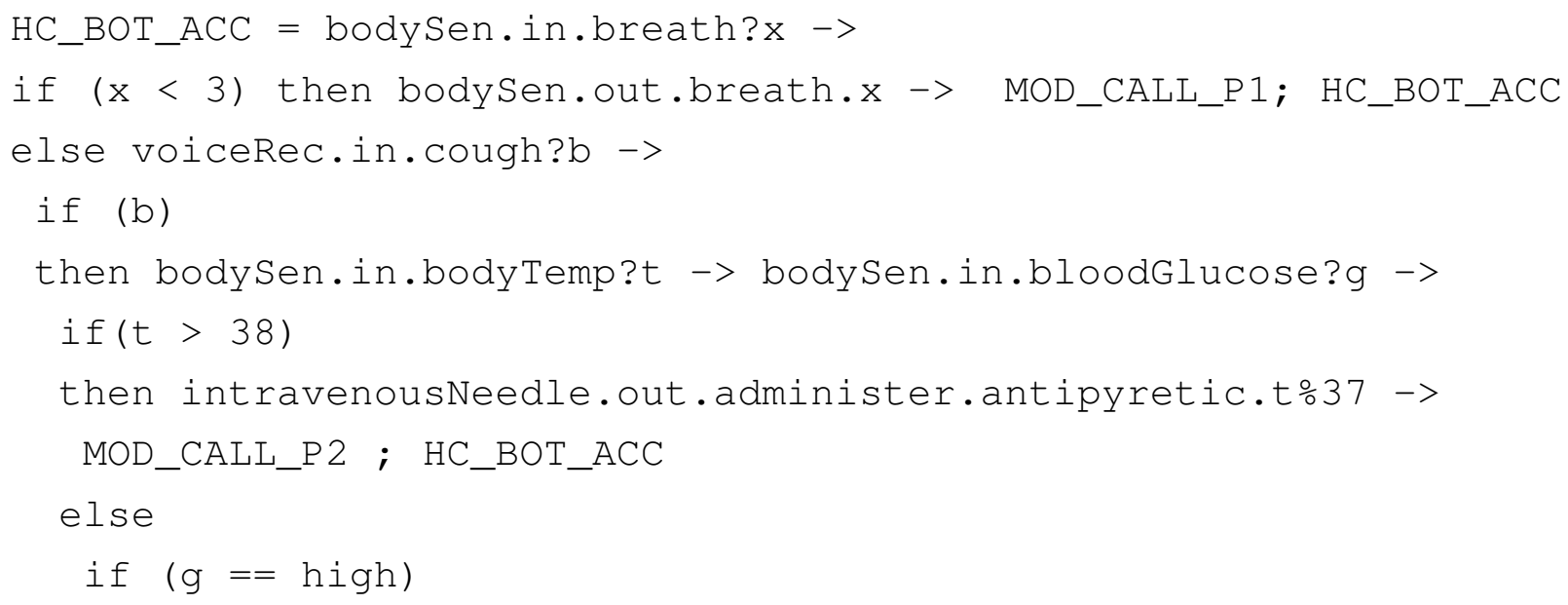




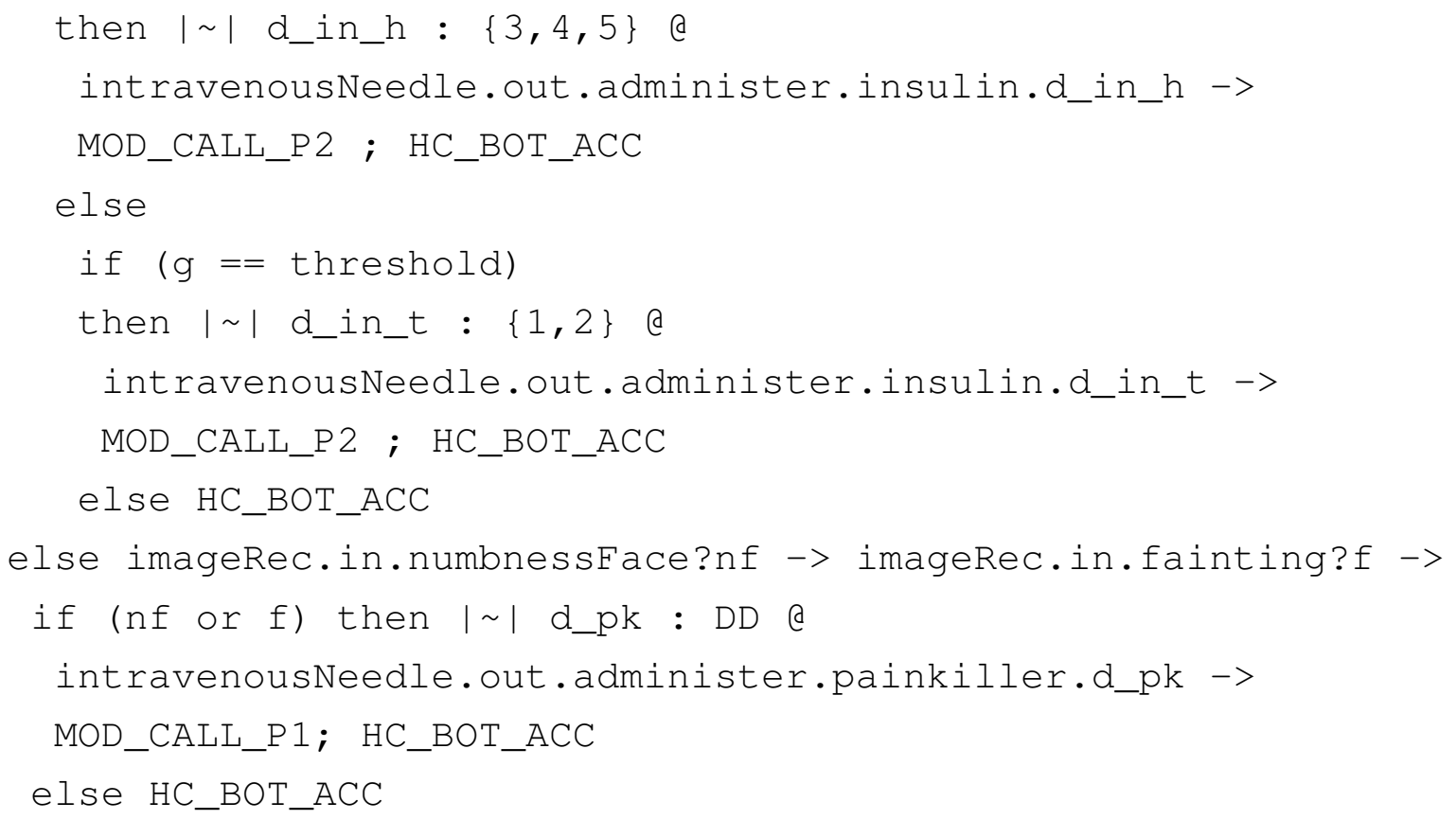

In $\mathcal{B R J} \mathcal{C}$, this extension is defined by the contract $C t_{H C \_B O T \_A C C}$. This healthcare robot version has a better (more deterministic) decision-making mechanism on the drug dose to be administered to the patient it monitors.

$$
\begin{aligned}
& C t r_{H C \_B O T \_A C C} \widehat{=} \\
& \left\langle\text { HC_BOT_ACC, }\left\{\begin{array}{l}
\text { bodysen } \mapsto \text { I_BS, imageRec } \mapsto \text { I_IS, } \\
\text { voiceRec } \mapsto \text { I_VS, phone } \mapsto \text { I_PH, } \\
\text { intravenousNeedle } \mapsto \text { I_IVN }
\end{array}\right\},\left\{\begin{array}{l}
\text { I_BS, I_IS, } \\
\text { I_VS, I_PH, } \\
\text { I_IVN }
\end{array}\right\},\right. \\
& \text { \{bodySen, imageRec, voiceRec, phone, intravenousNeedle\} }
\end{aligned}
$$

By Definition 3.1, we have that $C t r_{H C \_B O T} \sqsubseteq_{\mathcal{B}} C t r_{H C \_B O T \_A C C}$ : both components share the same channels with equivalent types (interfaces) and have their behaviours related by failures refinement $\mathrm{HC} \_\mathrm{BOT} \sqsubseteq_{\mathrm{F}} \mathrm{HC} \_\mathrm{BOT} \_\mathrm{ACC}$; it can be verified by the FDR assertion assert HC_BOT [F= HC_BOT_ACC.

The $C t r_{H C \_B O T \_A C C}$ brought some improvements to $C t r_{H C \_B O T}$. Nevertheless, the addition of new functionalities (or the enhancement of the existing ones) cannot be always addressed by refinement, even if we hide the implementation details before trying to establish such a relation. The component we present next, $C t r_{H C \_B O T \_T K}$, extends (inherits from) $C t r_{H C \_B O T \_A C C}$ with the addition of a talk module, which allows this robot to ask patients about their symptoms and thus can possibly better help them.

The I/O process HC_BOT_TK $\left(C t r_{H C \_B O T \_T K}\right.$ behaviour) improves HC_BOT_ACC by being able to interact with patients via the voice simulation/recognition device through the new channel talk. Together with the events bodySen.in.breath?x, it offers, initially, the possibility of behaving as MOD_TALK: it receives a chat request (talk.in.ask. lst), then 
collects information about chest discomfort (talk. in. chestDiscomfort?cd), headache (talk.in.headache? hd) and vision problems (talk.in.visionTrouble?vt). If the patient reports chest discomfort associated with headache or vision problems, the robot understands that a serious situation is under way and calls all the relevant individuals by behaving as MOD_CALL_P1. In any case, it goes to its initial state.

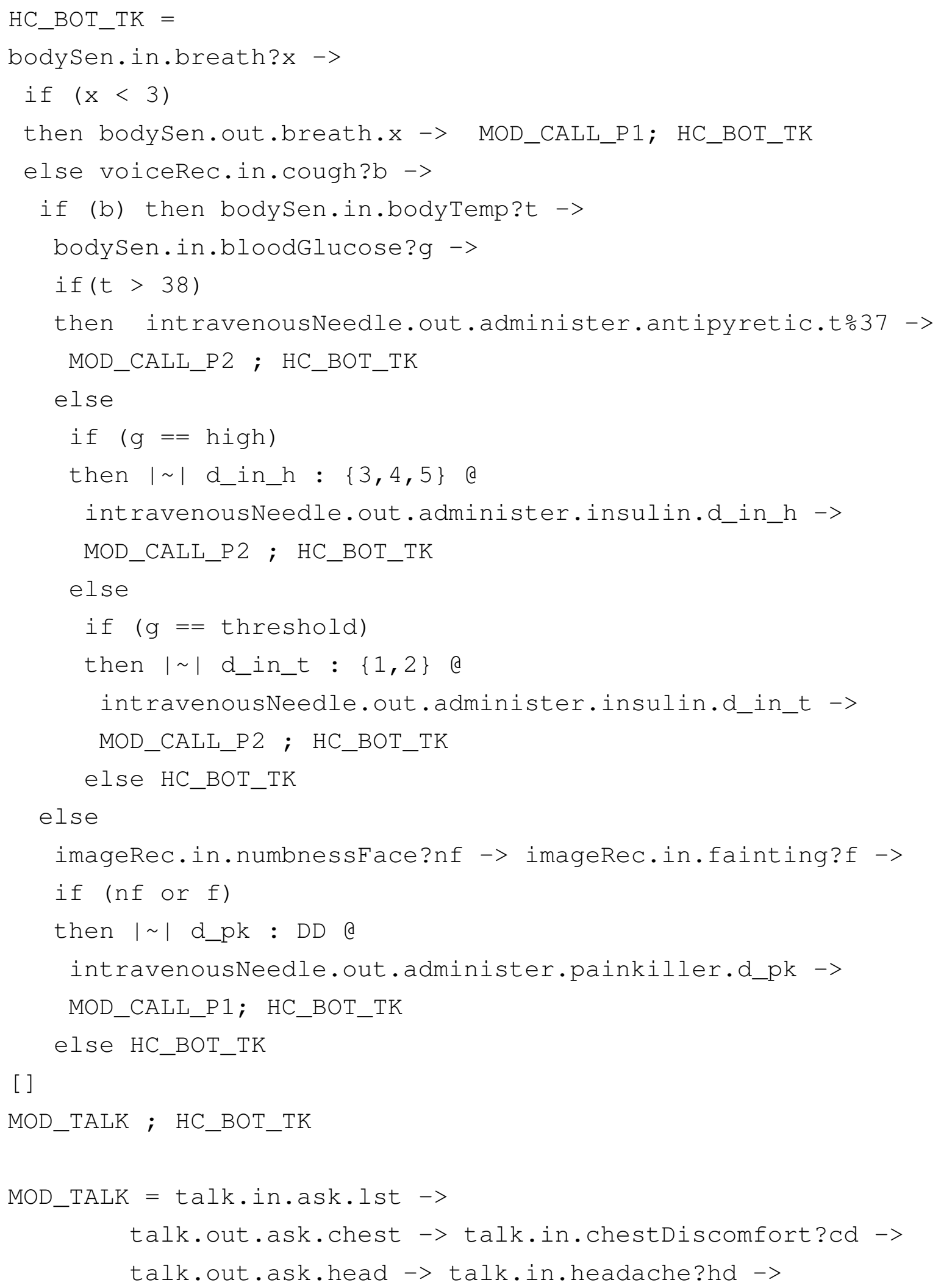




\section{talk.out.ask.vision -> talk.in.visionTrouble?vt -> if (cd and (hd or vt)) then MOD_CALL_PI else SKIP}

The $C t r_{H C \_B O T \_T K}$ contract is defined as bellow. By Definition 4.7, we have that $C t r_{H C \_B O T \_A C C}-_{e c v g} C t r_{H C \_B O T \_T K}$. Note that the attempt to stablish a failures relation between HC_BOT_ACC and HC_BOT_TK, provided the $\{|\mathrm{tal} \mathrm{k}|\}$ events are hidden on the latter, fail; it is shown by the FDR3 assertion HC_BOT_ACC $\left[\mathrm{F}=\mathrm{HC} \_\right.$BOT_TK $\backslash\{\mid$ talk $\mid\}$. This happens because the process C_BOT_TK $\backslash\{|\mathrm{talk}|\}$ can behave, initially, as the process MOD_CALL_P1, which is not possible for HC_BOT_ACC; therefore we have that the failure $(\langle\rangle, \Sigma \backslash\{$ phone.out.call.cNeighbor $\})$ belongs to $\mathcal{F}($ HC_BOT_TK $\backslash\{\mid$ talk $\mid\})$, but not to $\mathcal{F}\left(\mathrm{HC} \_B O T \_A C C\right)$. On the other hand, we know that HC_BOT_TK io_ecvg HC_BOT_ACC, which can be checked in FDR3 by the assertion Tester_ecvg (HC_BOT_ACC_serial) [F= HC_BOT_TK (Appendix A, Section A.2). This shows that convergence and inheritance, in the behavioural and component level perspectives, provide an entire new approach to evolve component-based specifications, whist preserving service conformance, which is stated in terms of a substitutability principle (WEGNER; ZDONIK, 1988).

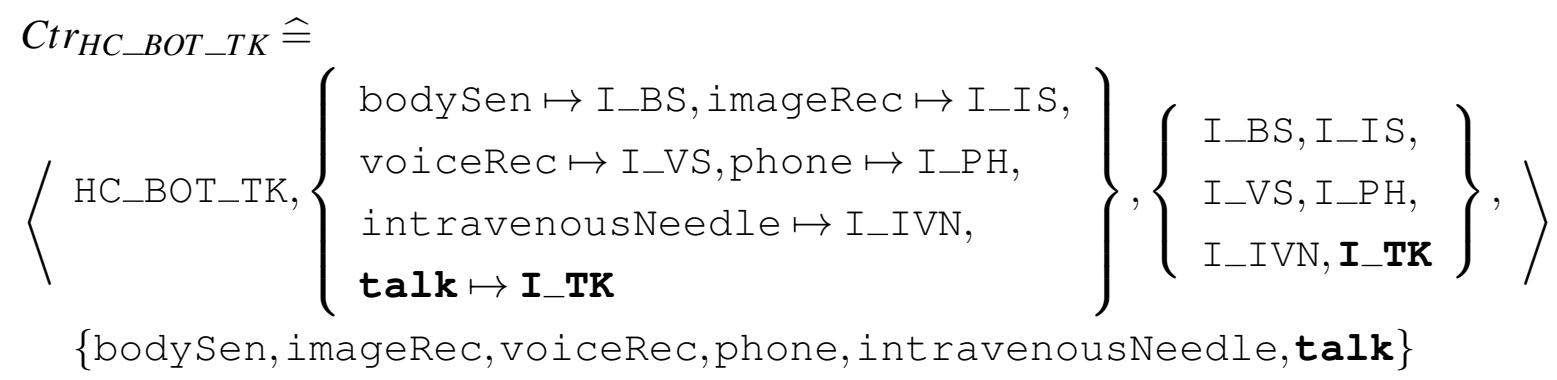

This case study is summarised by the component hierarchy depicted in Figure 5.4. In the next chapter, we revisit this specification to show how aspect oriented design can elegantly handle behavioural extensions, especially those scattered (crosscutting) through specifications, which is usually very hard to capture in conventional modelling techniques. 
Figure 5.4: The healthcare autonomous system hierarchy

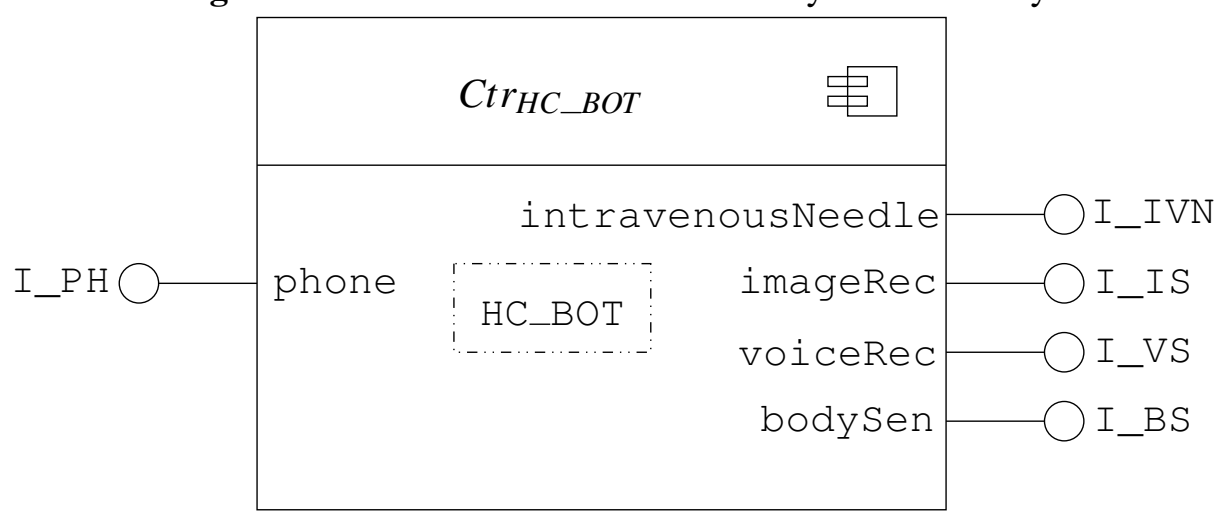

$\sqsubseteq_{\mathcal{B}}$

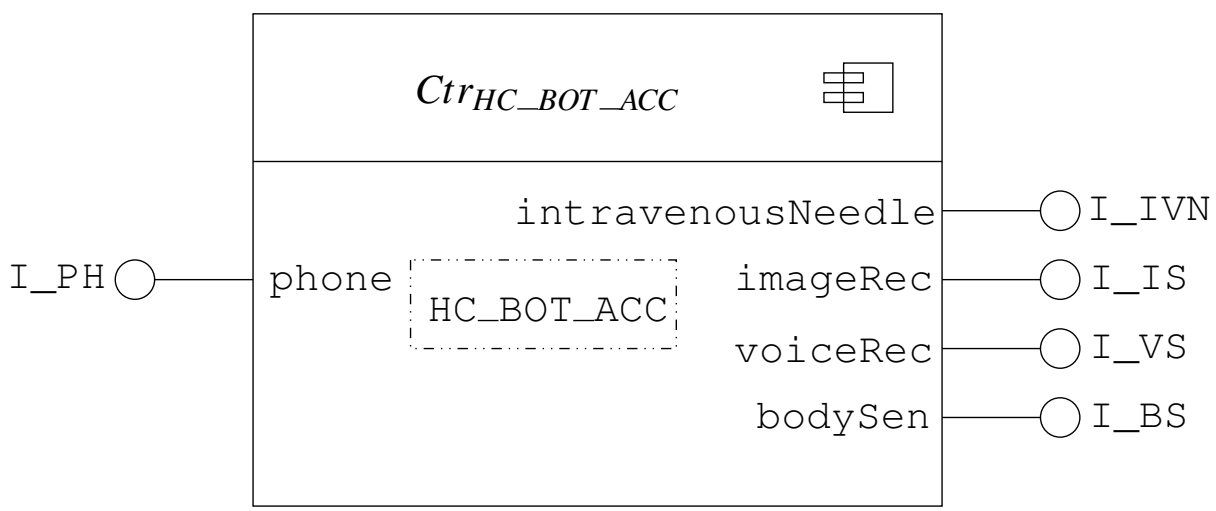

$\frac{1}{2}$

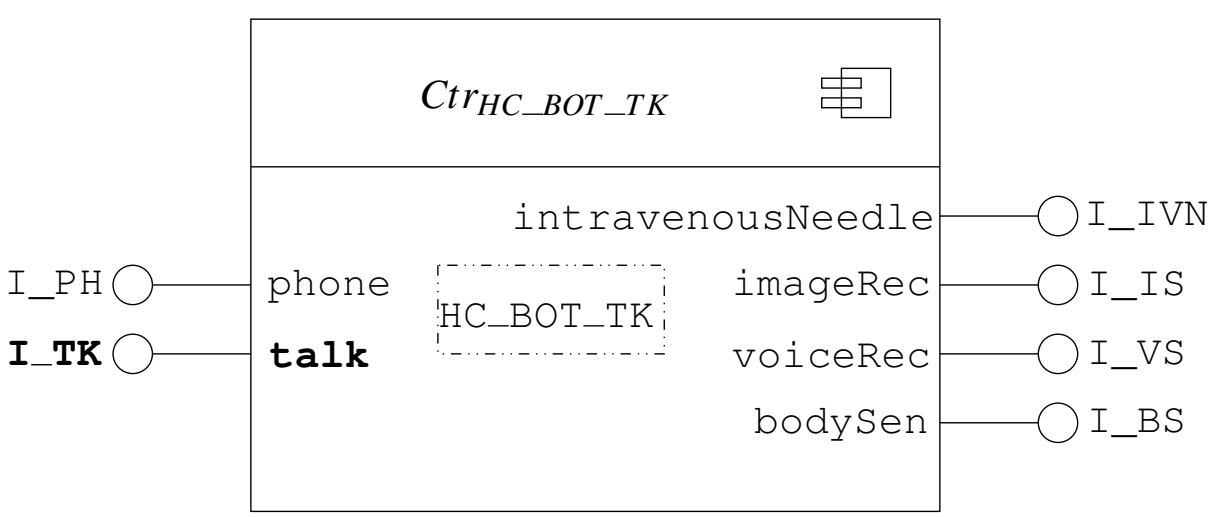

Source: José Dihego 


\section{P2P: Gnutella protocol}

A P2P (peer-to-peer) network is a set of interconnected nodes that alternate between the roles of client and server, with a decentralized control (SCHOLLMEIER, 2001; STEINMETZ; WEHRLE, 2005); nodes can join and leave the network freely (dynamic membership). The way in which nodes are connected defines the network topology, roughly hierarchical organisation (nodes form clusters based on their capabilities) or flattered (nodes are treated equally, despite their power of processing, storage and bandwidth).

Gnutella (CLIP2, 2001) is a well known implementation of a completely decentralised, flattered P2P network, designed primarily for file sharing; files are discovered by a distributed search protocol. In the current version of Gnutella (KLINGBERG; MANFREDI, June 2002), nodes perform tasks normally associated with both clients and servers, by which they are named servents (SERVer and cliENT). Gnutella is highly fault-tolerant, so if servents leave or enter the network, it keeps functional, although the resources diminish or increase, respectively.

The Gnutella protocol (CLIP2, 2001; KLINGBERG; MANFREDI, June 2002) defines four types of messages (payload type) servents can use to stablish connections, to request and send file shares:

- ping: used to actively discover hosts on the network. A servent receiving a ping is expected to respond with at least one pong;

- pong: the response to a ping. It includes the address of a connected Gnutella servent;

- query: the primary mechanism for searching the distributed network. A servent receiving a query will respond with a queryHit if a match is found against its local data set or will forward it to its neighbours;

- queryHits: the response to a query. It provides the recipient with enough information to acquire the data matching the corresponding query.

Quite briefly, a servent candidate $S$ joins the network by sending ping messages to servents in that network; a subset of them, say $S S$, can acknowledge with pongs, then $S$ can choose $S S^{\prime} \subseteq S S$ as its neighbours. If $S$ wants a file share, it sends a query to each $S S^{\prime}$ servents, they can respond with the information needed to acquire the requested file share, if they own it; or retransmit such request to its respective neighbours, and so on. This forward is bounded: a package has a defined time-to-live ( $T T L$ : the number of times the package will be forwarded by Gnutella servents before it is removed from the network) and can take a limited number of HOPS (the number of times the package has been forwarded). Therefore, a forward decrements $T T L$ and increases $H O P S$, according to:

$$
\begin{aligned}
& \operatorname{TTL}_{(i)}+\operatorname{HOPS}_{(i)}=T T L_{(0)} \\
& \operatorname{TTL}_{(i+1)}<T T L_{(i)}
\end{aligned}
$$




$$
\operatorname{HOPS}_{(i+1)}>\operatorname{HOPS}_{(i)}
$$

where $T T L_{(i)}$ and $\operatorname{HOPS}_{(i)}$ represent the value of $T T L$ and HOPS in the $i^{- \text {th }}$ hop, respectively. Each active package is uniquely identifiable by a GUID, which comprises the servent id NODID (where the package was created) and the local message id MSGID (which identifies packages locally).

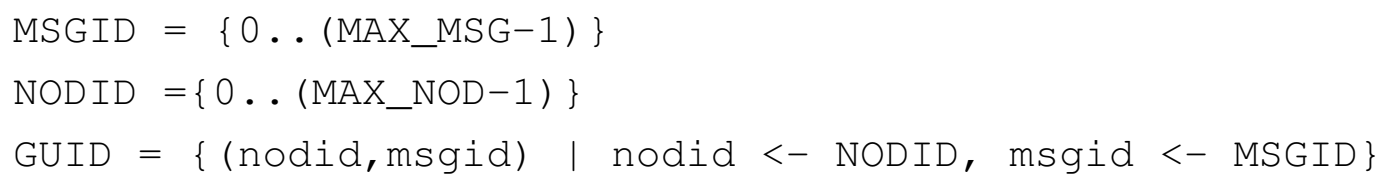

A package (PACK) has a header (HEADER) and a body message (MSGBODY), provided both have the same payload type (PayloadType). A package header has a unique identifier (GUID), a payload type (ping, pong, query or queryHit), the current timeto-live ttl (TTL) and the number of hopes hops (HOPS) it has taken so far, provided (ttl+hops) == MAX_TTL. The constant MAX_TTL must be respected by the servents in a Gnutella network; it guarantees fairness and enforce limits on traffic.

The message body caries a payload type (pingBody, pongBody,queryBody or queryHitBody) and maybe some associated data. The message body for a ping has no additional data; a NODID is sent in a pong to inform the source of such package; a query has the file id and share index it is looking for; a query hit carries the servent id destination and the requested share. This mechanism of acquiring a share is a simplification of the original protocol, where a query hit carries information needed to establish a direct connection between who asked for/provide the share. Therefore, in our simplified model, servents never communicate directly. PACK $=\{(h, m) \mid h<-$ HEADER, $m<-\operatorname{MSGBODY}, \operatorname{sameType}(h, m)\}$

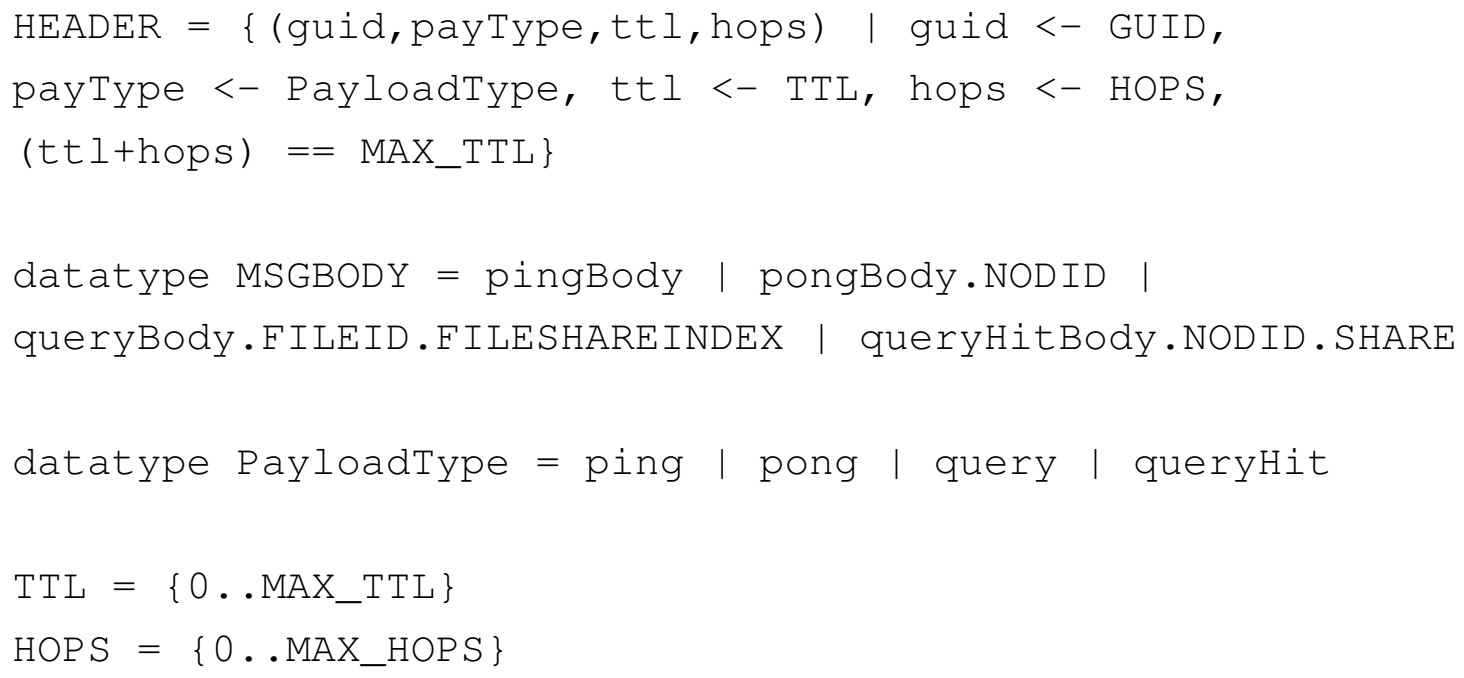

Files are divided into shares, which are the resource provided/requested by servents. A servent can access a file if it owns its shares on local storage. A share (SHARE) has the file id it belongs to (FILEID), an index (FILESHAREINDEX) and a content (CONTENT). 


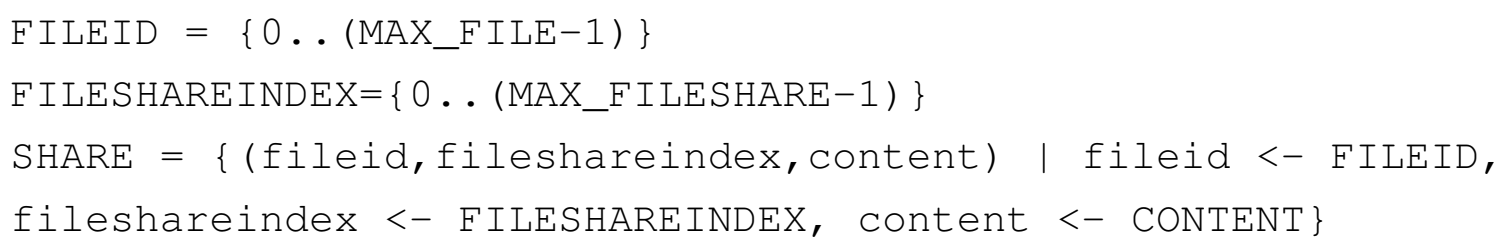

Servents communicate using two channels, send and receive. Before being sent, packages are tagged with out (OUT_PACK), and expected to be received with the tag in (IN_PACK). Through the channel status the servent communicates events like package drop (drop.PayloadType), income cache is full (full), it is blocked to send (bloked) or it is waiting for packs (waiting.PayloadType).

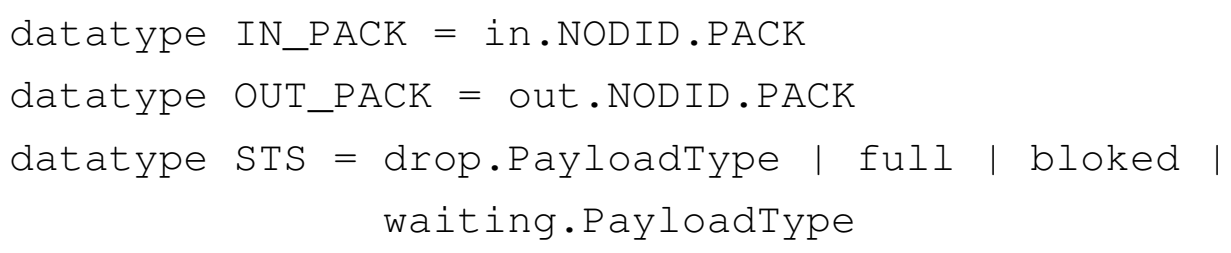

channel send : OUT_PACK

channel receive : IN_PACK

channel status : STS

Servents cache incoming packages, therefore there is no guarantee that a ping will be immediately responded with a pong, for example. We factor the Servent I/O process according to the states it can pass through, just to list two: (1) the servent will handle pings in its cache, responding to them with pongs; (2) the servent will examine cached queries, responding to them with a query hit when possible, forwarding them to its neighbours or dropping them because their time-to-live have reached zero. These auxiliary processes (including Servent itself) are parametrised by: a unique servent id ( $\mathrm{ni}$ ), a local package index (mi), a set of cached packages (ps), a set of local file shares ( $\mathrm{f} f \mathrm{~s}$ ) and how many other servents it knows are alive (alv). All functions used in the following processes can be found in Appendix B.

The I/O process SND_PING checks whether the servent has sent the maximum number of pings (MAX_MSG) it has the right to. If not it sends a ping package and behaves as Servent with package counter $\mathrm{mi}$ incremented (modulo MAX_MSG); otherwise it blocks from sending pings (status.bloked) and behaves as Servent.

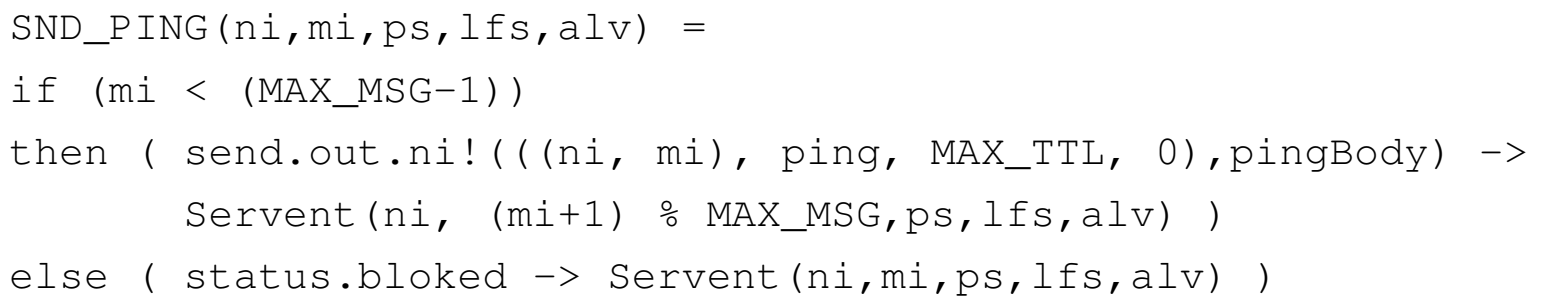

A servent can request shares by behaving as SND_QUERY. It sends a query package with the maximum time-to-live MAX_TTL and, conversely, the minimum value for hops, then behaves as Servent (package counter incremented modulo MAX_MSG); the missing shares 
queryset ( $l f s$ ) have the same priority of acquisition, therefore, they are given the same odds of being requested by SND_QUERY through a non deterministic choice. If it owns all file shares available in the network, SND_QUERY blocks from sending more queries and behaves as Servent.

SND_QUERY (ni, mi,ps, lfs, alv) =

if (not empty (queryset (lfs)))

then ( $|\sim|$ q : queryset (lfs) a

( send.out.ni!(( (ni,mi), query,MAX_TTL, 0), queryBody.q)

$->$ Servent (ni, $(m i+1)$ ․ MAX_MSG, ps, lfs,alv) ) )

else ( status.bloked $\rightarrow$ Servent (ni,mi,ps, lfs,alv) )

If a servent has cached pings (not empty ( $\operatorname{select}(\mathrm{ping}, \mathrm{ps})$ )), it might respond them with pongs. A pong package must be sent to ping p's source servent get_N $(p)$, with the maximum time-to-live MAX_TTL and, conversely, the minimum value for hops. If $\mathrm{p}$ has reached the furthest servent it is allowed to $\left(g e t \_T(p)==0\right.$ and get_H $(p)==$ MAX_HOPS), then it is dropped (status.drop.ping); otherwise it is forwarded (send.out.ni! fwd (p)) and then, in both cases, SND_PONG behaves as Servent with package p removed from its cache (diff (ps, $\{\mathrm{p}\}))$.

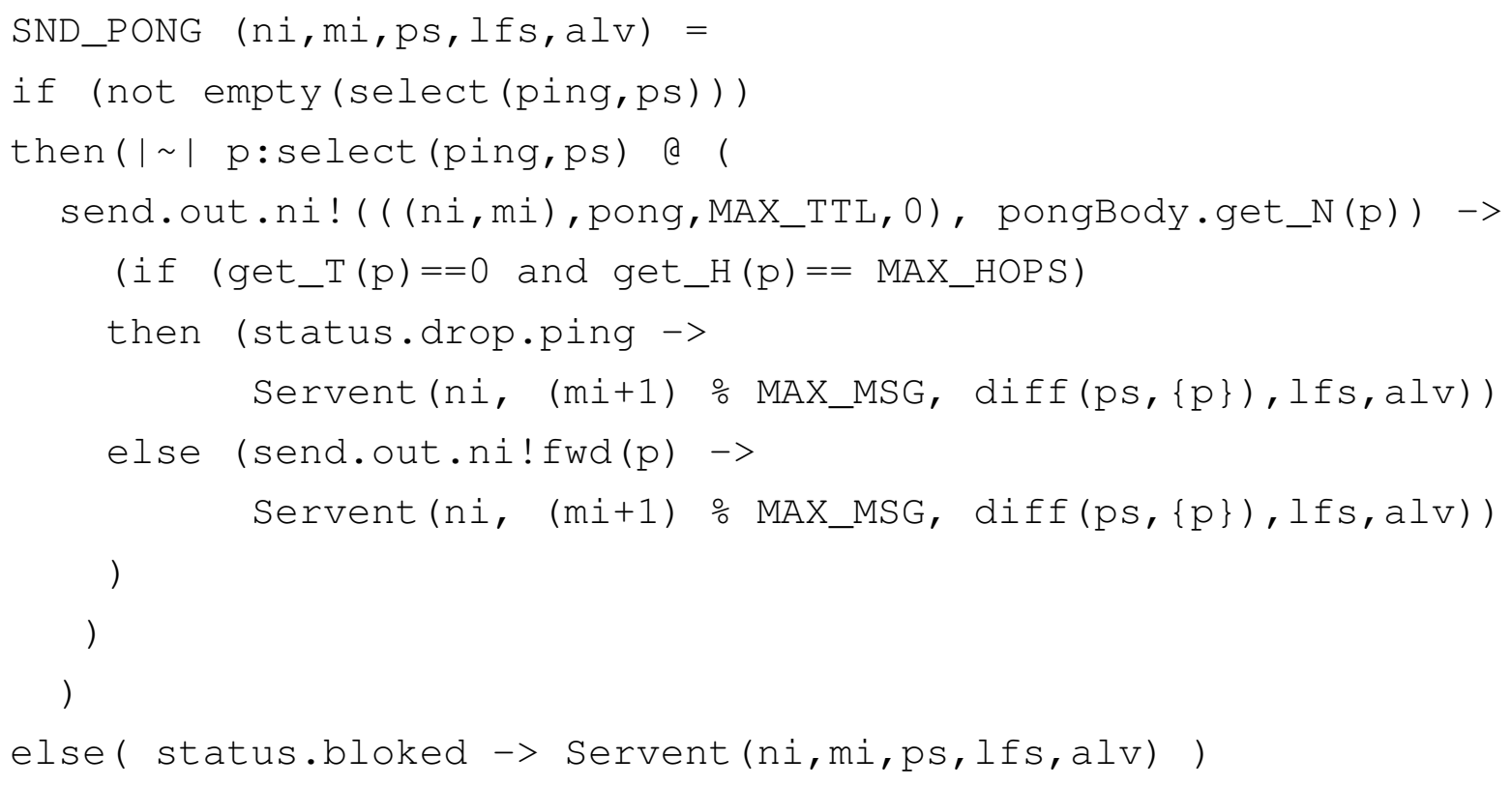

When a servent has queries to respond (not empty ( I/O process SND_QUERYHIT searches the local storage for a matching file share (search (get_QI (mb), get_QS (mb), Ifs) ) and replies with a query hit if it succeeds. Otherwise, it drops the query p (status. drop.query) because it has reached the furthest possible servent or time-to-live is not zero and SND_QUERYHIT forwards $p$ (send. out. $n i$ ! fwd $(p)$ ). In any case it behaves as Servent with package $p$ removed from its cache $(\operatorname{diff}(p s,\{p\}))$. SND_QUERYHIT (ni, mi,ps, lfs, alv) = if (not empty (select (query,ps))) 


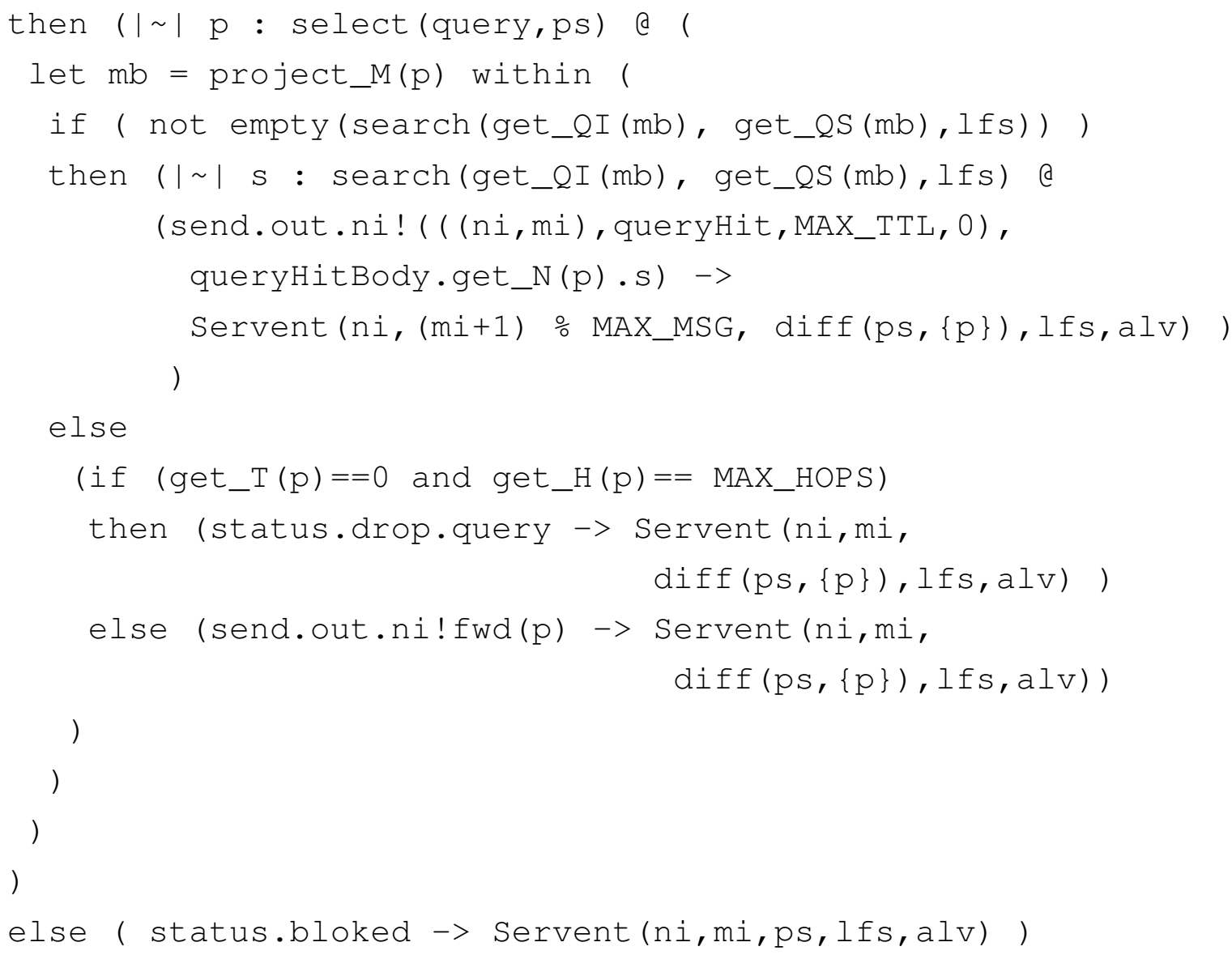

We handle the cached pongs with two I/O processes: PROC_PONG and FWD_PONG. The intent of PROC_PONG is to process pongs while counting the number of nodes that have responded to the servent pings (incrementing a lv counter). Apart from that, both processes behave similarly: they select a cached pong $\mathrm{p}$ and check whether it must be dropped because its time-tolive has reached zero (status.drop.pong) or if p must be forwarded; then, in both cases, FWD_PONG behaves as Servent with package $p$ removed from its cache $(\operatorname{diff}(p s,\{p\}))$.

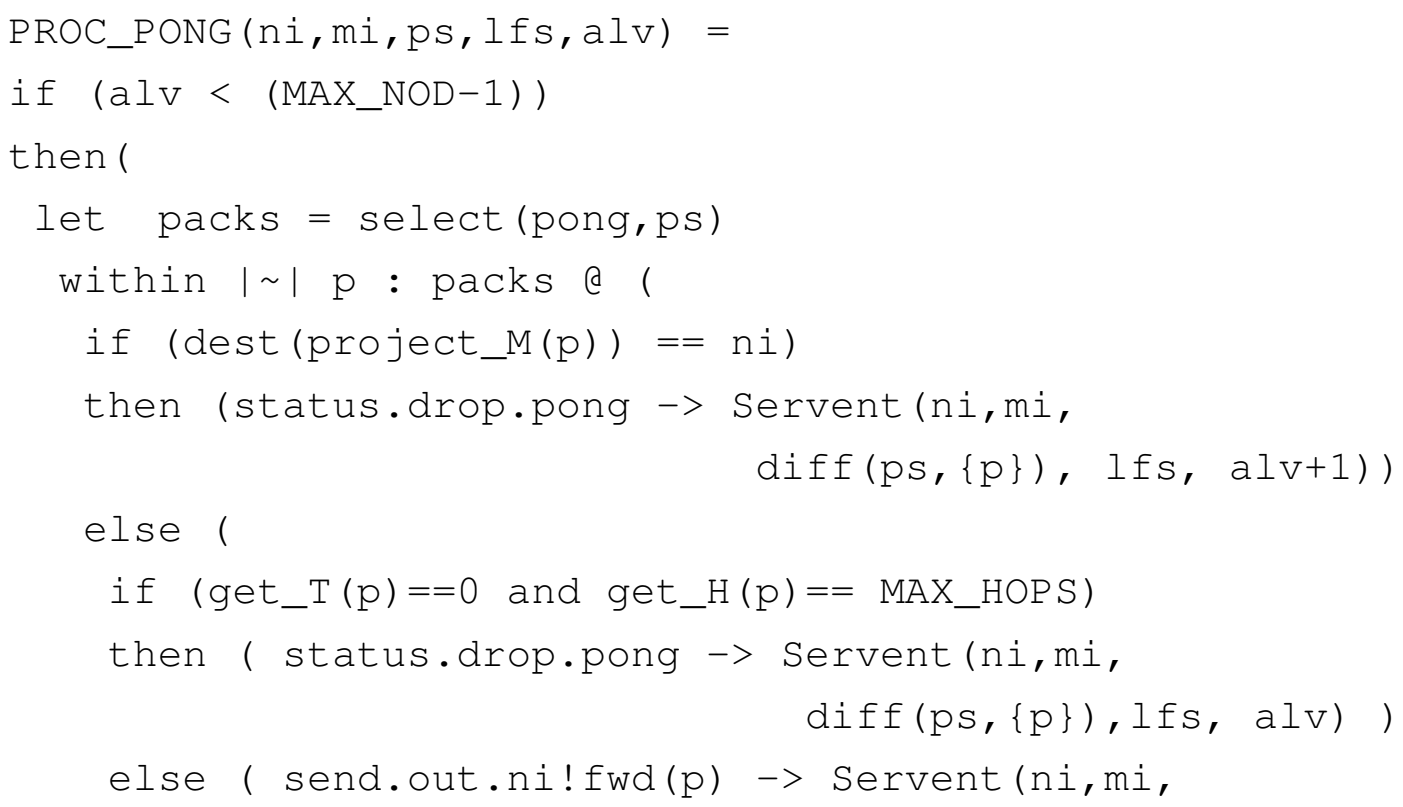




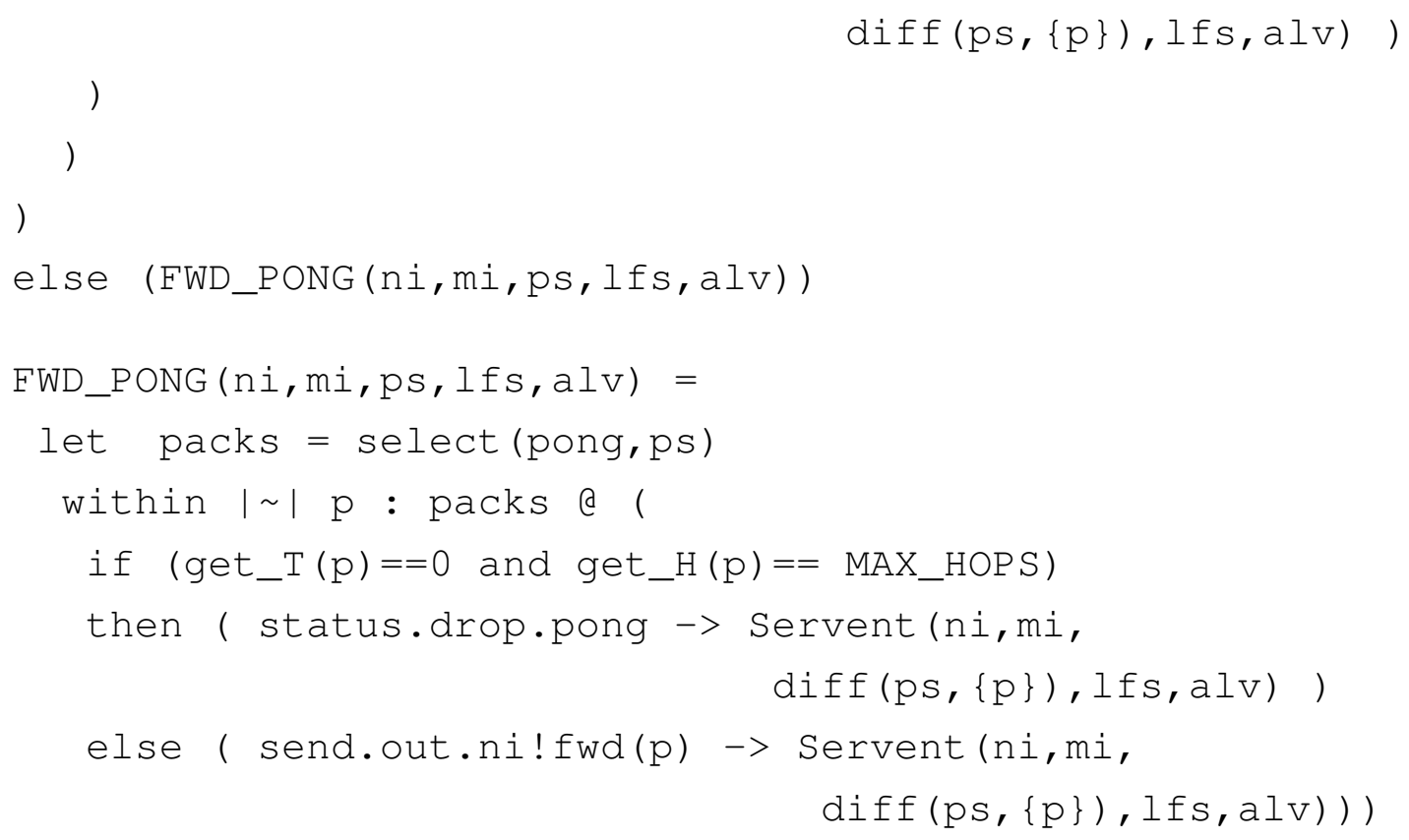

Queries are responded by query hits, which are processed by PROC_QUERYHIT. If servent $\mathrm{n} i$ is the final destination of a query hit $\mathrm{p}$ (dest (project_M $(\mathrm{p}))==\mathrm{n} i$ ), the file share component of $\mathrm{p}$ is extracted (get_SH (project_M (p)) ) and stored locally (union ( Ifs, \{get_SH (project_M(p)) \})). Otherwise, the package p is dropped (status. drop. queryHit) if it has no more time-to-live or, forwarded (end. out. ni ! fwd (p) ). In any case, PROC_QUERYHIT behaves as Servent with $\mathrm{p}$ removed from cache.

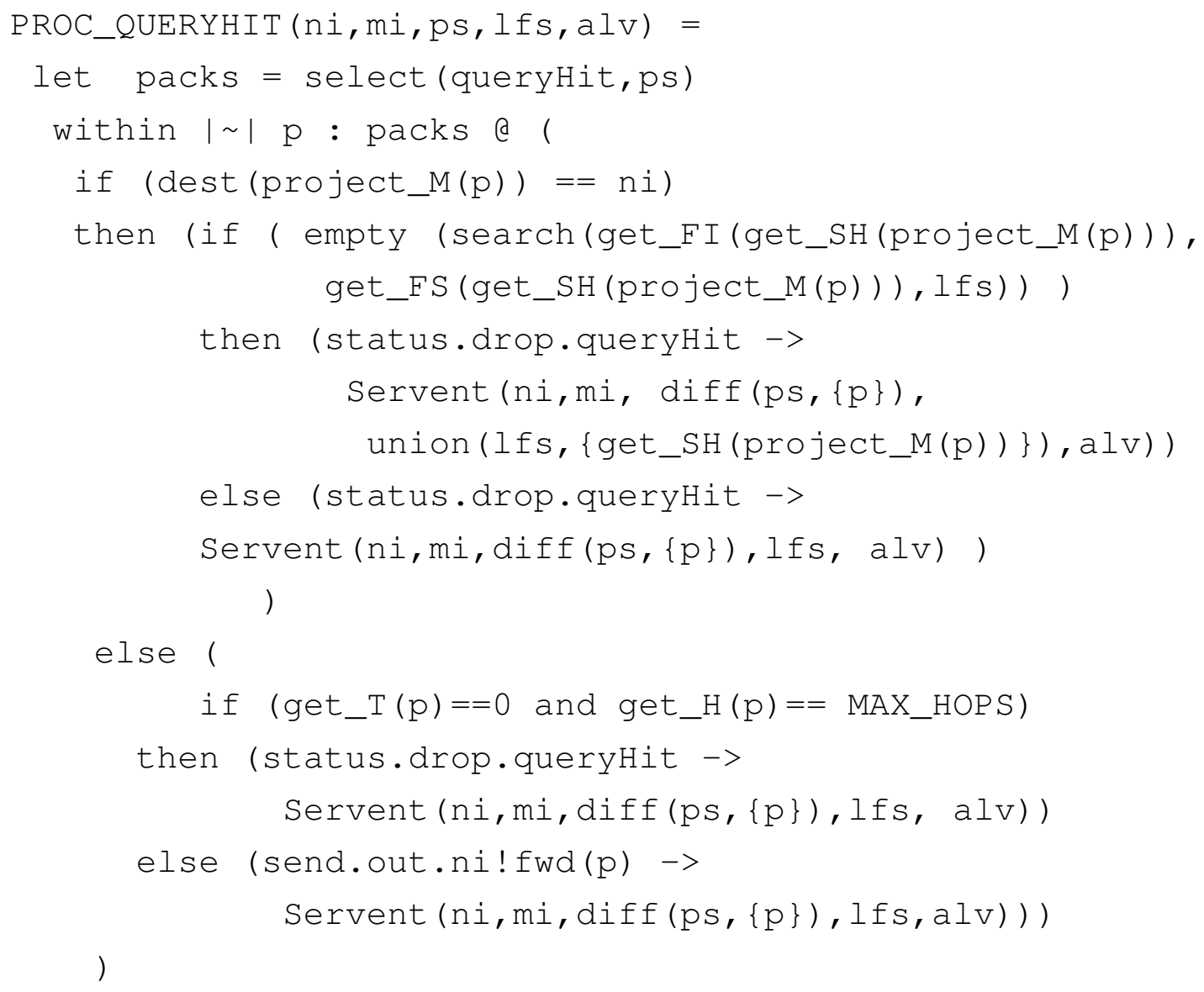


The role of receiving incoming packages is the responsibility of the I/O processes RCV_PING_PONG and RCV_ALL. In the set up phase, before the servent has reached a minimum quorum (quorum ( $(\mathrm{lv})$ ), RCV_PING_PONG receive only pings and pongs. If the minimum quorum of active servents is reached, RCV_ALL accepts any incoming package. Both processes accept packages provided the cache is not full ( $\operatorname{card}(\mathrm{ps})<\mathrm{CACHE}$ _SIZE).

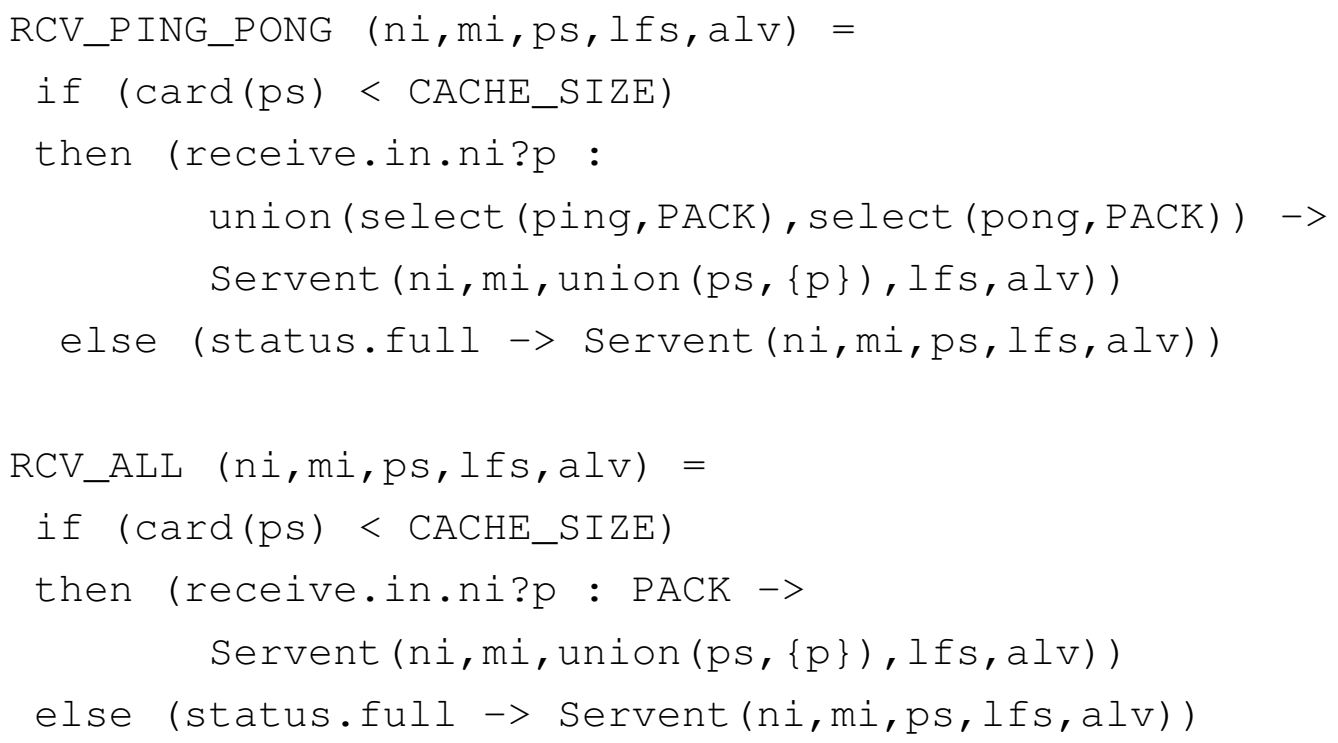

The I/O process Servent orchestrates the previous processes we introduced. In the set up phase (not quorum $(a l v)$ ) it can only send/receive pings or pongs, until it knows a minimum number of servents are active in the network. After the set up phase, Servent can (a) send queries (SND_QUERY), reply to pings with pongs (SND_PONG and FWD_PONG), reply to queries with query hits (SND_QUERYHIT) and update its local storage with incoming file shares (PROC_QUERYHIT) or (b) it can receive all kinds of incoming packages (RCV_ALL).

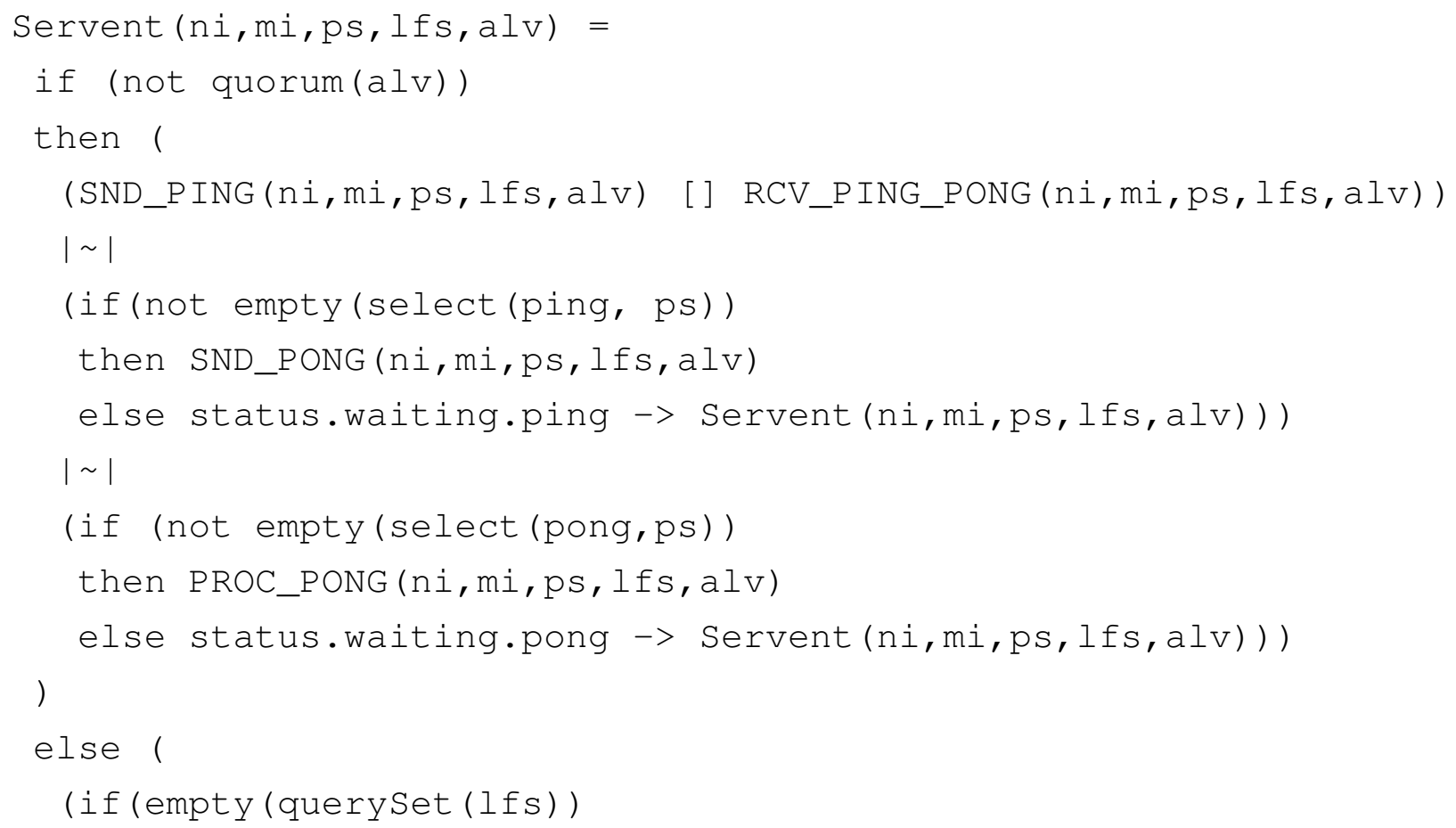




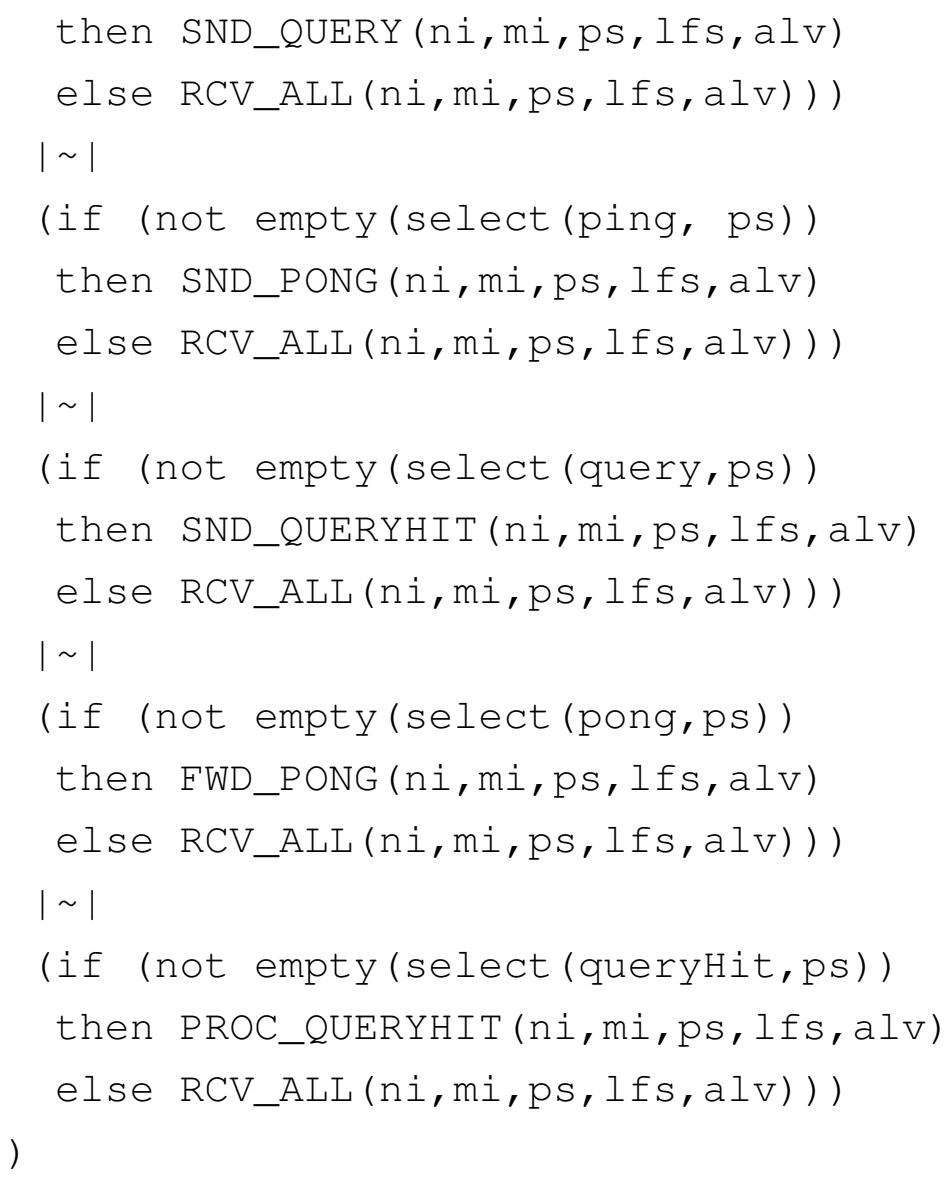

To become fully operational (capable of sending and receiving any king of packages), a servent must, in the set up phase, discover a minimum number of active servents in the network; if such a network is low connected and/or the time-to-live is under the optimal value (an optimal value allows all servents to become fully operational and satisfy their queries in a finite time), servents can benefit if they can send pings and forward pongs one more time than the expected, so they can have more chances to become fully operational.

We implement this mechanism by adding the input channel last Shot, and redefining (in a convergent way) the processes SND_PONG, PROC_PONG and Servent. When ready to drop a ping $\mathrm{p}$, the process SND_PONG' offers the input event last Shot and forwards one more time $\mathrm{p}$ (send.out. $\mathrm{ni}$ ! $\mathrm{fwd}(\mathrm{p})$ ). The same reasoning applies to the process PROC_PONG', where a pong can be, instead of dropped, forwarded a last time. The process Servent' is obtained from Servent by replacing the original helper processes for these primed versions.

channel last Shot

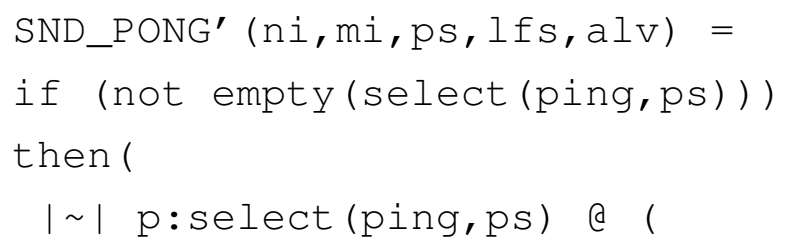




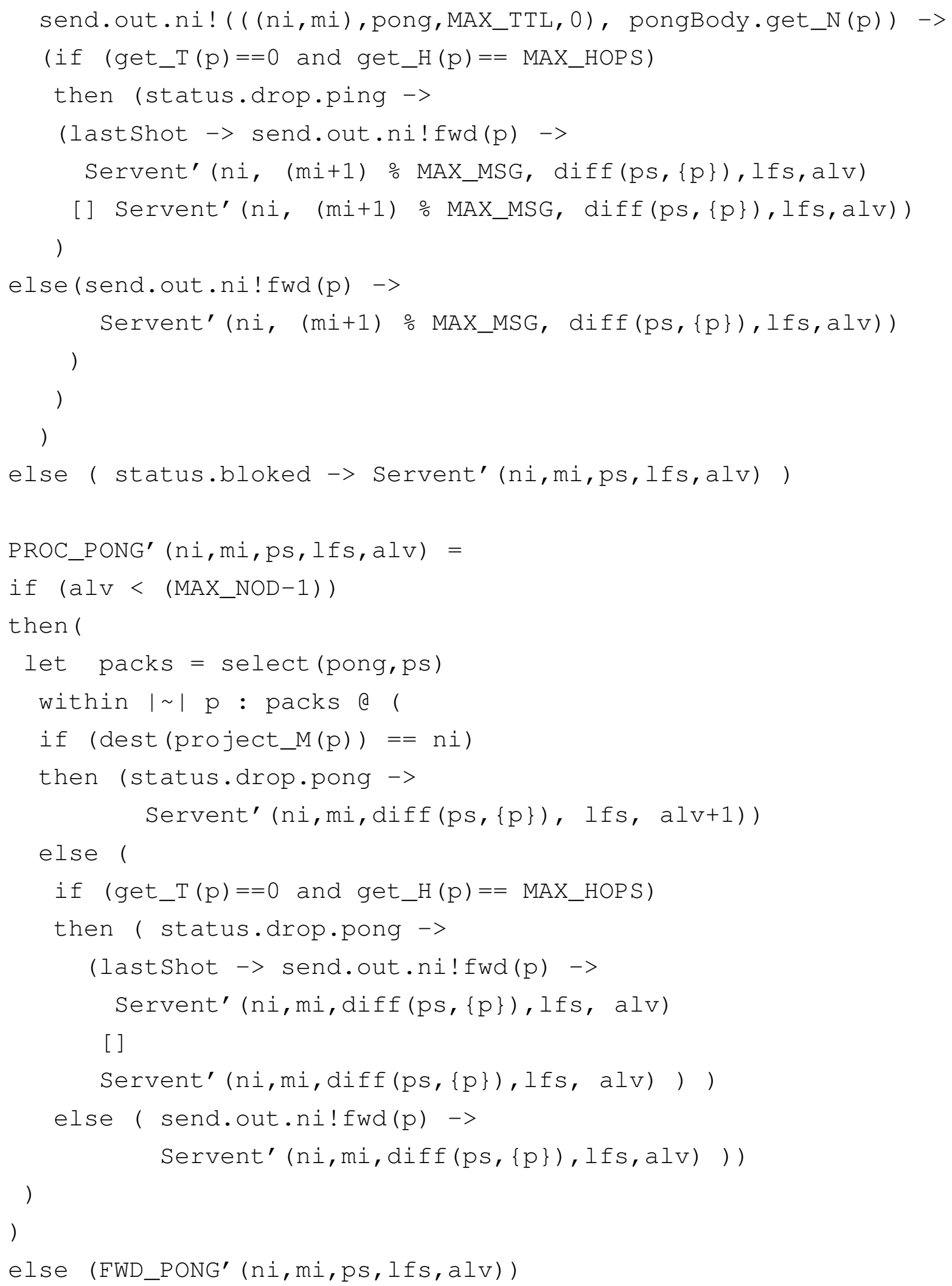

Figure 5.5 summarises and concludes this case study. The component $C_{t} r_{\text {Servent }}$ inherits

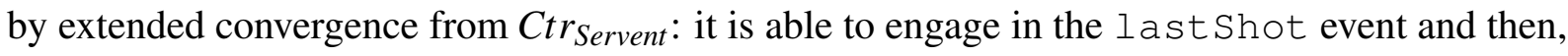
forward pings/pongs one more time than allowed by TTL, which increases the connections between nodes and decreases the overall traffic of query/queryHit packages. 

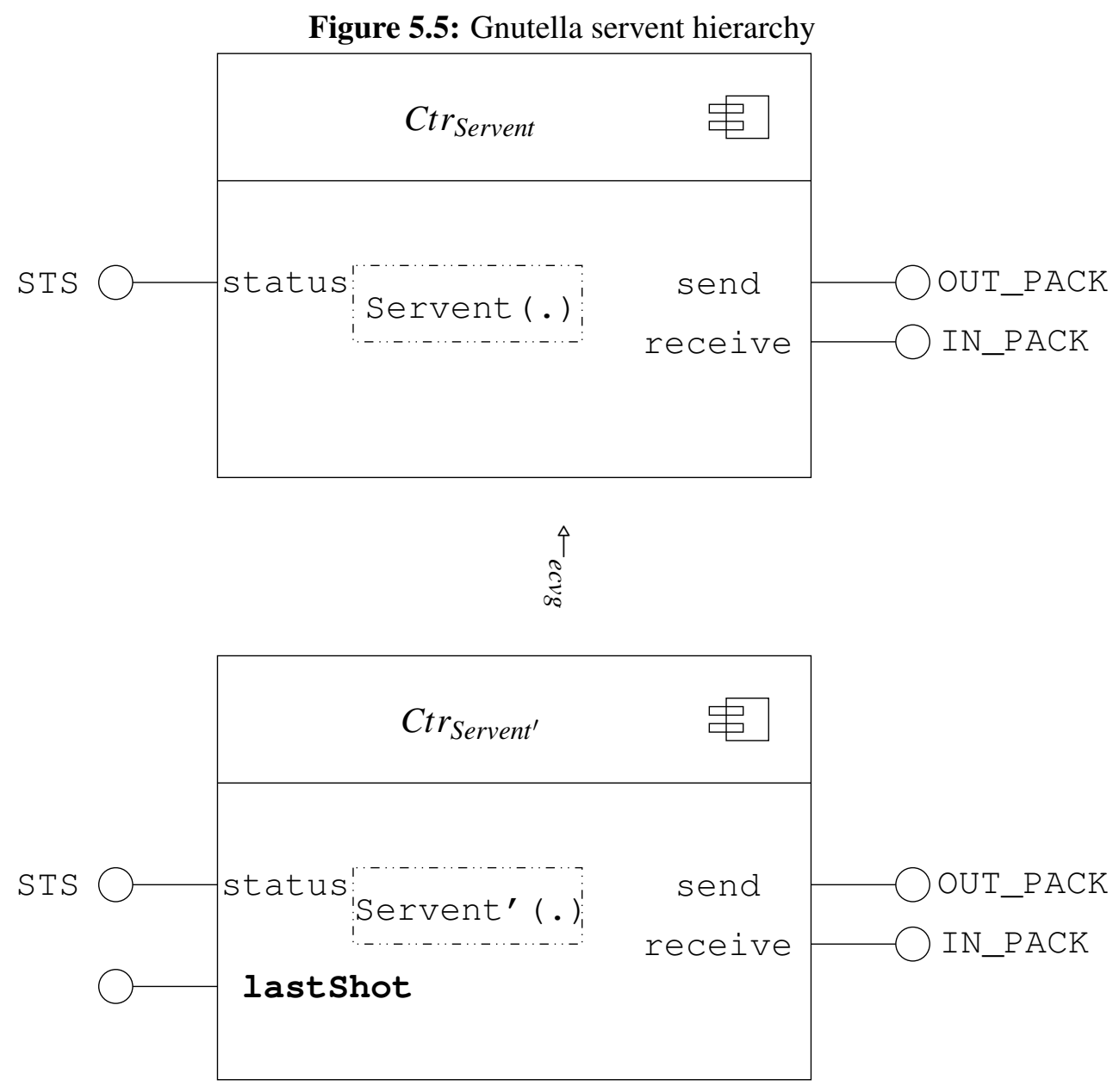

Source: José Dihego 


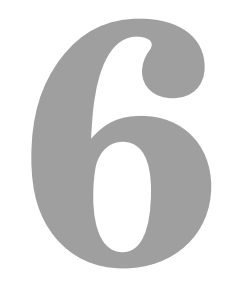

\section{Aspect-oriented development in BRIC}

Aspect-oriented programming (AOP) (KICZALES et al., 1997) has been presented as a theory to capture, define and modularise crosscutting concerns, which spread through applications. Aspects are hard to modularise by using conventional programming units, such as functions or classes, because they tend not to be units of the system's functional decomposition, but rather to be properties that affect the semantics of regular components (KICZALES et al., 1997). Some aspects are so recurring that have become classical examples of when and how applying AOP: context-sensitive behaviour, performance optimizations, monitoring, logging, persistence, distribution, security and transactional management, just to name a few. Successful AOP technologies are used both in industry and academy, as AspectJ (KICZALES et al., 2001) for Java and AspectC (COADY et al., 2001) for the C language.

Aspect-oriented programming is part of a broader development strategy, Aspect-Oriented Software Development (AOSD) (FILMAN et al., 2004), which brings aspect-oriented analyses to early phases of software development. The incorporation of aspect-oriented design (AOD) in the modelling phase creates a more accurate and natural correspondence between specification and implementation, benefiting costly development activities as testing and maintenance.

A promising scenario that we investigate here is the integration of AOSD and componentbased model driven development (CB-MDD) (SZYPERSKI, 1998), a well recognised approach to develop complex systems, which are built from simpler ones, called components, with welldefined interface and behaviour. As far as we are aware, there is no work that explores the integration between AOD and CB-MDD formal approaches, which is one of the goals of this thesis (DIHEGO; SAMPAIO, 2015).

We contribute by formalising how to capture, model and weave aspects with components in the $\mathcal{B R J C}$ component model (RAMOS; SAMPAIO; MOTA, 2009), which, as we have seen in Chapter 2, formalises the core CB-MDD concepts and, moreover, supports compositions, where behavioural properties, such as deadlock freedom, are ensured by construction. Our aspectoriented design (AOD) approach safely supports extensions of $\mathcal{B R J \mathcal { C }}$ components by preserving a notion of service conformance given in terms of our convergence relations (Definition 4.2 and 4.4) that are suitable to capture model evolution. 
Aspect-Oriented Design enhances CB-MDD by providing a modular mechanism to model orthogonal properties, such as, but not limited to, persistence, logging and access control. Without AOD such aspects tend to be scattered/replicated over components, which reduces maintainability, reuse and readability; these are the main reasons for integrating AOD to CBMDD.

To get an intuition how aspects can enhance CB-MDD approaches, specifically $\mathcal{B} \mathcal{R J C}$, recall the TV remote control specification in Section 2.2. There, we developed two I/O processes related by convergence, TV_RC' ecvg TV_RC, which, along with their channels and interfaces, can be encapsulated in $\mathcal{B R J C}$ components related by inheritance, $C t r_{\mathrm{TV} \_\mathrm{RC}} \leftarrow_{e c v g} C t r_{\mathrm{TV} \_\mathrm{RC}}$ (Definition 4.7). The component $C t r_{\mathrm{TV} \_ \text {RC' }}$ extends $C t r_{\mathrm{TV} \_\mathrm{RC}}$ by providing a menu for video settings adjustments (contrast and brightness). Suppose the TV cable provider adopts a new system where channels are classified based on viewers age (general, parent guidance and adult, for example). It decides to update the software on both its setup box and remote control, such that every time a user (Granpa or Boy) tries to access a adult channel, a warning message is displayed, giving some inadvertent child a chance to jump out that inappropriate content or to provide a password and watch.

The question is, how can we implement this new functionality on $C t r_{\mathrm{TV} \_\mathrm{RC}}$ and $C t r_{\mathrm{TV} \_\mathrm{RC}}$, such that the resulting components are still related by inheritance. More importantly, consider the context where the TV cable provider changes its mind and decides to expand its categories, embodying a system of channel ranking and user profile filters. With the current tools on the $\mathrm{CB}-\mathrm{MDD}$ approaches, specifically in the case of $\mathcal{B} \mathcal{R J C}$, the path is to update the behaviour of each component, merging new functionalities by hand and then rechecking inheritance. In our specific example, let $\mathrm{MC}$ be the list of adult channels and pass be the default TV provider password. We must identify each time TV_RC (and TV_RC') communicates $\mathrm{ch}$, followed by up or down and then check whether the next channel belongs to $\mathrm{MC}$ and, in such a case, ask for a password, which must be equal to pass.

The previous solution is poor maintainable, error prone and needs inheritance/refinement rechecking on each update. Suppose we have at our disposal a mechanism to define a behavioural pattern and the extensions we want to be placed every time our model matches such a pattern. In terms of the TV remote control model, we want to identify each time the I/O process TV_RC (and TV_RC') communicates ch followed by up or down, such that it navigates to a adult channel. In those points we want to display a warning message and ask for a password. If it is possible to pack this behaviour pattern and extension into a single unit and then integrate it with both $C t r_{\mathrm{TV} \_\mathrm{RC}}$ and $C t r_{\mathrm{TV} \_\mathrm{RC}}$, such that we preserve the inheritance relation between the resulting components, then we have a better solution in terms of maintainability. This scenario is what we present in this chapter: an aspect-oriented design to $\mathcal{B} \mathcal{R J C}$ in which we can capture and integrate extensions into our component models in a maintainable, reusable and compositional way.

This chapter is based on our results presented in (DIHEGO; SAMPAIO, 2015) and is 
organised as follows: in Section 6.1 we establish how to define and weave aspects into componentbased specifications. In Section 6.2 we prove that aspects guarantee service conformance by establishing how it relates with behavioural convergence and component inheritance. In Section 6.3 we illustrate our results by a case study of an autonomous healthcare system we have developed in Chapter 5.

\section{Aspect-oriented modelling for BRIC}

In the aspect oriented paradigm, a join point is a well-defined point in a program execution, established in terms of its control flow and/or predicates over its state space. A pointcut stands for a collection of join points grouped by a common predicate. We can consider a join point as the result of a behavioural pattern matching, a dynamic entity that serves the purpose of identifying the points where advices (behaviour modifiers) can be incorporated. An advice is an action (behaviour) that is designed to be offered on a set of related join points, grouped into a pointcut. The process of causing the relevant advice to be offered on join points is called weaving, which is by definition a dynamic process (WAND; KICZALES; DUTCHYN, 2004).

In our AOD approach for $\mathcal{B} \mathcal{R J C}$, a join point represents an I/O process (Definition 2.4) stable state, one without internal transitions leading out from it. A pointcut groups a set of related join points. An advice is an I/O process that is designed to be weaved with $\mathcal{B} \mathcal{R J C}$ components at a set of join points highlighted (marked) in their behaviour. An aspect is a pair formed of an advice and a pointcut, which expresses the intention to weave such an advice into the pointcut join points. The fact that we have a common language for describing component behaviour, pointcuts and advices has a tremendous impact on the complexity of our aspect-component weaver (ACW) (KICZALES et al., 1997). This task, ultimately, is reduced to combine CSP processes, which benefit from an extensive set of operators (ROSCOE, 1998).

A pointcut raises a set of join points that need to be marked, so the ACW (presented later in this section) is able to identify where advices must be plugged. We use as marks the events communicated through a special kind of channel, a check mark channel, say, $c$, which communicates only two events, $c . b f$ and $c . a f$; these are offered sequentially ( $c . b f$ then $c . a f$ ) acting as join point marks (Definition 6.1). The event $c . b f$ must be an input; so, according to the input determinism property (Definition 2.4), a decision to mark a join point is always predictable (deterministic). Intuitively, $c . b f$ stands by the event that appears just before the advice behaviour is offered and c.af, conversely, the event communicated immediately after it.

Definition 6.1 (Check mark channel). Consider an I/O channel $c$. We say that $c$ is a check mark channel if and only if: $\{|c|\}=\{c . b f, c . a f\} \wedge c . b f \in$ inputs.

To get an intuition how our AOD concepts integrate with $\mathcal{B R J \mathcal { C }}$, we consider the component $C \operatorname{tr}_{\mathrm{SRV}}=\left\langle\mathrm{SRV},\left\{\mathrm{SrV} \mapsto \mathrm{I} \_\mathrm{DTSER}\right\},\{\right.$ I_DTSER $\left.\},\{\mathrm{SrV}\}\right\rangle$. The CSP process SRV offers 
Figure 6.1: Marking join points
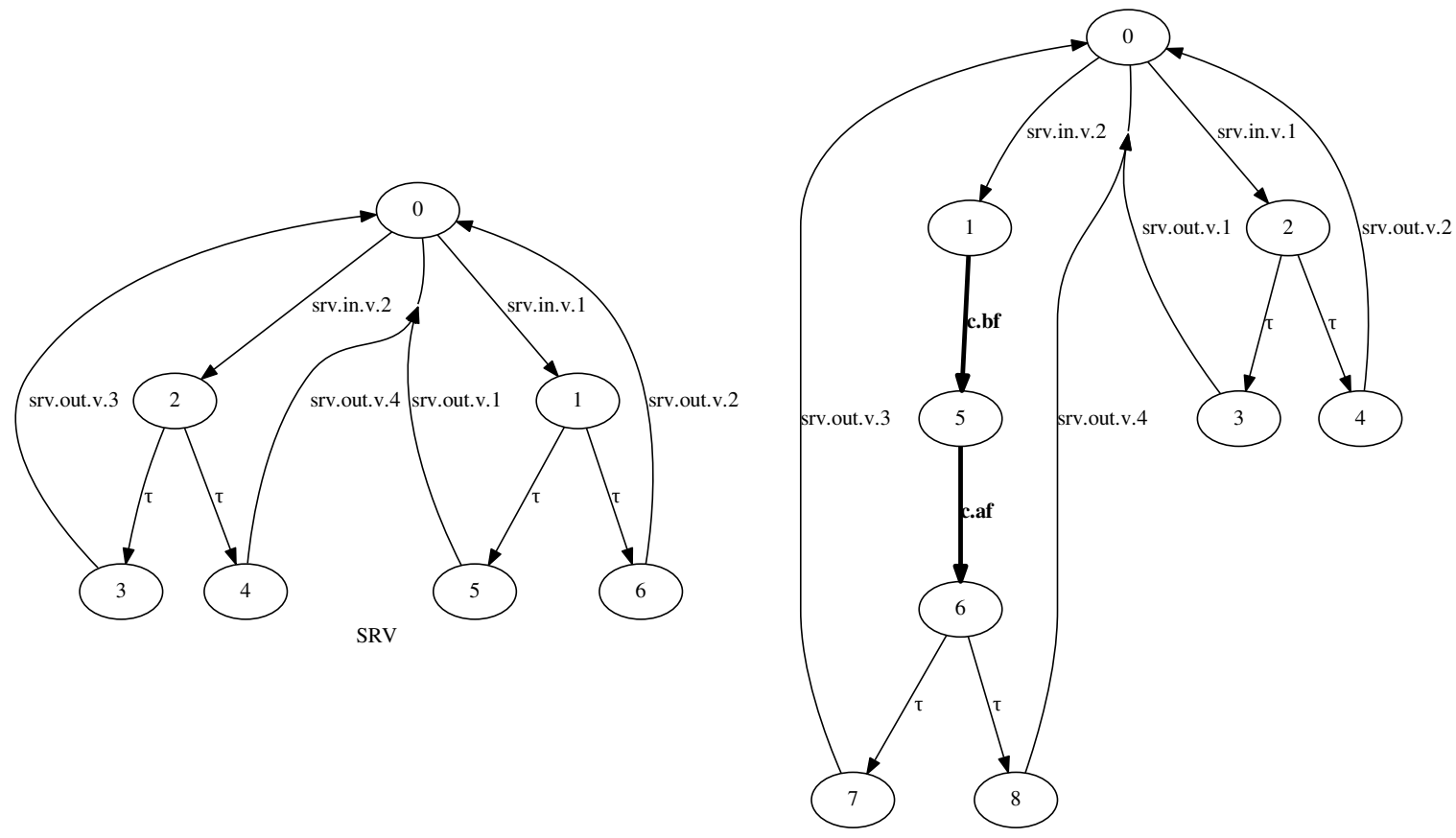

Source: José Dihego

the environment the choice between srv.in.v. 1 and srv.in.v.2; if it synchronises on the first then SRV communicates, non deterministically, srv. out.v. 1 or srv. out.v. 2 and recurses. The same reasoning applies in the case the environment synchronises on srv.in.v.2. The I/O channel srv has the type I_DTSER, where SUB_INT is a finite subset of $\mathbb{N}$; the tags in and out distinguish input from output events and $v$ indicates the communication of a SUB_INT value.

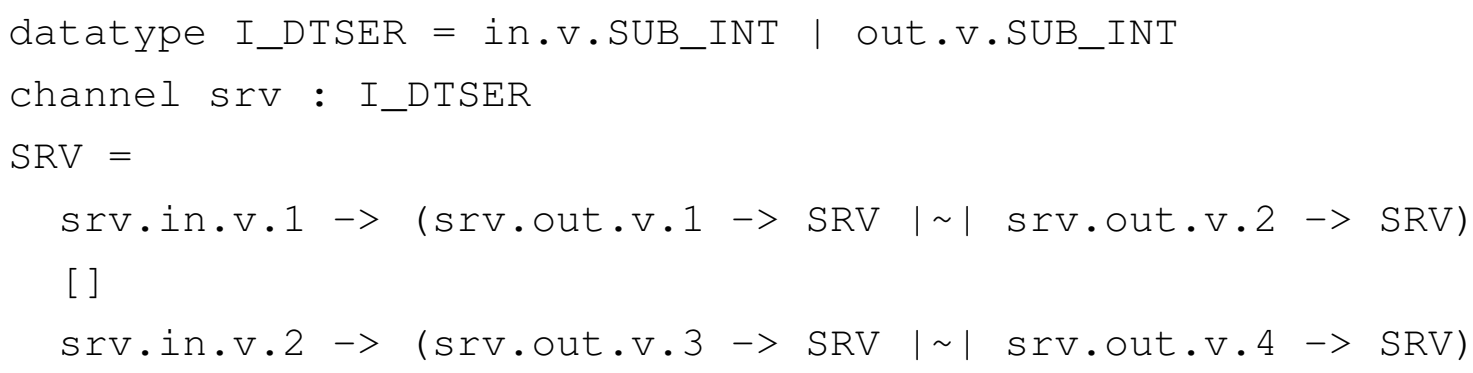

Suppose we want to mark, for logging purposes, every time SRV communicates SrV . in . v. 2 followed by srv.out.v. 3 or srv.out.v.4. Figure 6.1 shows the SRV's LTS (for visualisation purposes) and the resulting process after marking the described join point with the events communicated through the check mark channel $c:\{b f, a f\}$.

The pattern and the mark events used to define and signalise join points are encapsulated in a pointcut designator (or just a pointcut, Definition 6.2). It is defined by a CSP process $P$, which marks join points by offering the events of a check mark channel $c$. For example, consider the process $\mathrm{P} 1$ defined as follows: 


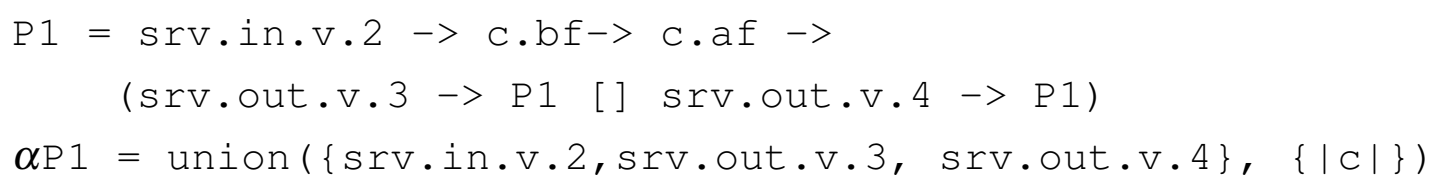

The LTS depicted on the right-hand side of Figure 6.1 is the result of the pointcut $p:\langle\mathrm{P} 1, \mathrm{c}\rangle$ application over SRV. After engaging in srv.in.v.2, P 1 does $\mathrm{c} . \mathrm{bf}$ and $\mathrm{c} \cdot \mathrm{af}$ sequentially, then offers the choice between srv.out.v.3 and srv.out.v.4, returning, in either case, to its initial state. Note that $\mathrm{P} 1$ is not an I/O process (it does not satisfy the strong output decisiveness property of Definition 2.4 by offering outputs in external choice), although its composition with SRV (SRV [|diff $(\alpha P 1,\{|c|\}) \mid] \quad P 1)$ is, as we can see by analysing the LTS of the composition on the right-hand side of Figure 6.1. The process $\mathrm{P} 1$ acts as a monitor of SRV; it synchronises with SRV on its alphabet ( $\alpha \mathrm{P} 1)$, except for the mark events $\{|\mathrm{C}|\}$, which are performed each time the monitored process matches the pointcut pattern described by $\mathrm{P} 1$.

Definition 6.2 (Pointcut designator). Consider a check mark channel $c$ and a CSP process $P$. We say that $p c d:\langle P, c\rangle$ is a pointcut designator if and only if:

(i) $P$ is deterministic and deadlock-free;

(ii) $\forall t \in \operatorname{traces}(P) \bullet t \downarrow c . a f \leq t \downarrow c . b f \leq t \downarrow c . a f+1$. P never communicates more $c . a f$ events than $c . b f$ events, and neither do they fall more than one behind;

(iii) $\exists t^{\wedge}\langle c . b f\rangle \in \operatorname{traces}(P) \Rightarrow\left(t^{\wedge}\langle c . b f\rangle, \Sigma \backslash\{c . a f\}\right) \in$ failures $(P)$. If $P$ communicates $c . b f$ then it rejects everything but c.af;

(iv) $\exists t^{\wedge}\langle c . b f\rangle \in \operatorname{traces}(P) \Rightarrow(t, \Sigma \backslash\{c . b f\}) \in$ failures $(P)$. If $P$ can communicate $c . b f$ then it rejects everything but $c . b f$.

According to Definition 6.2, given a component behaviour $\mathcal{B}$ (which is an I/O process by Definition 2.3), $P$ performs $c . b f$ and $c . a f$, sequentially, every time $\mathcal{B}$ synchronises on its behavioural pattern. The behavioural properties of I/O processes, especially strong output decisiveness and input determinism, associated with the pointcut well-formed conditions allow us to state that:

(i) if $P$ offers, initially, a set of input events iset, then $\mathcal{B}$ matches $P$, if it offers, initially, at least one element of $i$ set;

(ii) if $P$ offers, initially, a set of output events oset, then $\mathcal{B}$ matches $P$, if it offers, initially, only a non-empty subset of oset;

(iii) inductively, the above holds for any subsequent state of $P$ and $\mathcal{B}$, provided $P \backslash\{|c|\}$ and $\mathcal{B}$ have performed the same trace. 
The check mark channel $c$ should only be used to mark join points, so $\{|c|\}$ (the set of events communicated through $c$ ), iset and oset are disjoint. Since $P$ is deterministic (ROSCOE, 1998): (i) is a consequence of input determinism; (ii) an implication of the strong output decisiveness property; (iii) emerges by the fact that the events $\{|c|\}$ are business meaningless, so $\{|c|\} \cap \alpha \mathcal{B}=\emptyset$. Therefore, for $\mathcal{B}$, it does not matter if $P$ engages in any event via channel $c$, so $\mathcal{B}$ is concerned only with the process $P \backslash\{|c|\}$, which behaves as $P$, where all events from $\{|c|\}$ are hidden from the environment.

We have established the means to define, locate and mark join points. Next, we discuss how to define the behaviour we want to weave at them. An advice (Definition 6.3) comprises an I/O process $A$ (obeying some well-formed conditions), which is designed to be weaved at join points marked by a check mark channel $c$ and to interact with its environment through a set of I/O channels $C$.

Definition 6.3 (Advice). Consider that A, $C$ and $c$ stand, respectively, for a CSP process, a set of I/O channels and a check mark channel. We say that $a d v:\langle A, C, c\rangle$ is an advice if and only if:

(i) $A \backslash\{|c|\}$ is an I/O process.

(ii) $c \notin C$ and $\alpha(A \backslash\{|c|\}) \subseteq\{|C|\}$. The events performed by $A$ are signalled in its interface, except those communicated through $c$;

(iii) $\forall t \in \operatorname{traces}(A) \bullet t \downarrow c$.af $\leq t \downarrow c . b f \leq t \downarrow c$.af +1 . A never communicates more $c$.af's than $c . b f$ 's, and neither do they fall more than one behind;

(iv) $\exists t^{\wedge}\langle c . b f\rangle \in \operatorname{traces}(P) \Rightarrow(t, \Sigma \backslash\{c . b f\}) \in$ failures $(P)$. If $P$ can communicate $c . b f$ then it rejects everything but c.bf;

(v) $\exists t^{\wedge}\langle$ c.af $\rangle \in \operatorname{traces}(P) \Rightarrow(t, \Sigma \backslash\{c . a f\}) \in$ failures $(P)$. If $P$ can communicate $c$.af then it rejects everything but c.af.

Definition 6.3 requires the advice visible behaviour $(A \backslash\{|c|\})$ to be an I/O process, which is not enforced on the pointcuts behaviour (Definition 6.2). This is because advices are designed to change how a component behaves by being weaved into its behaviour, which must be, by Definition 2.3, an I/O process; on the other hand, pointcuts are designed to just mark join points into components behaviour, leaving unchanged their external behaviour (the behaviour exhibited though their interface), therefore they do not need to behave as I/O processes.

Following our running example, consider the join points marked over the component $C t r_{\mathrm{SRV}}$ by the pointcut $p:\langle\mathrm{P} 1, \mathrm{C}\rangle$ (on the right-hand side of Figure 6.1). The advice $a$ : $\langle A,\{d b\}, C\rangle$ comprises the logging I/O process $A$, which is designed to be weaved at each join point marked by $\mathrm{C}$ and to interact with its environment by the channel $\mathrm{db}$. By communicating c.bf and c.af it knows where A must be weaved. The I/O process A communicates the event $\mathrm{db}$. out. save.v.2 to log in a database the fact that its target server $C_{\text {SRV }}$ has performed 
Figure 6.2: Weaving advices into join points
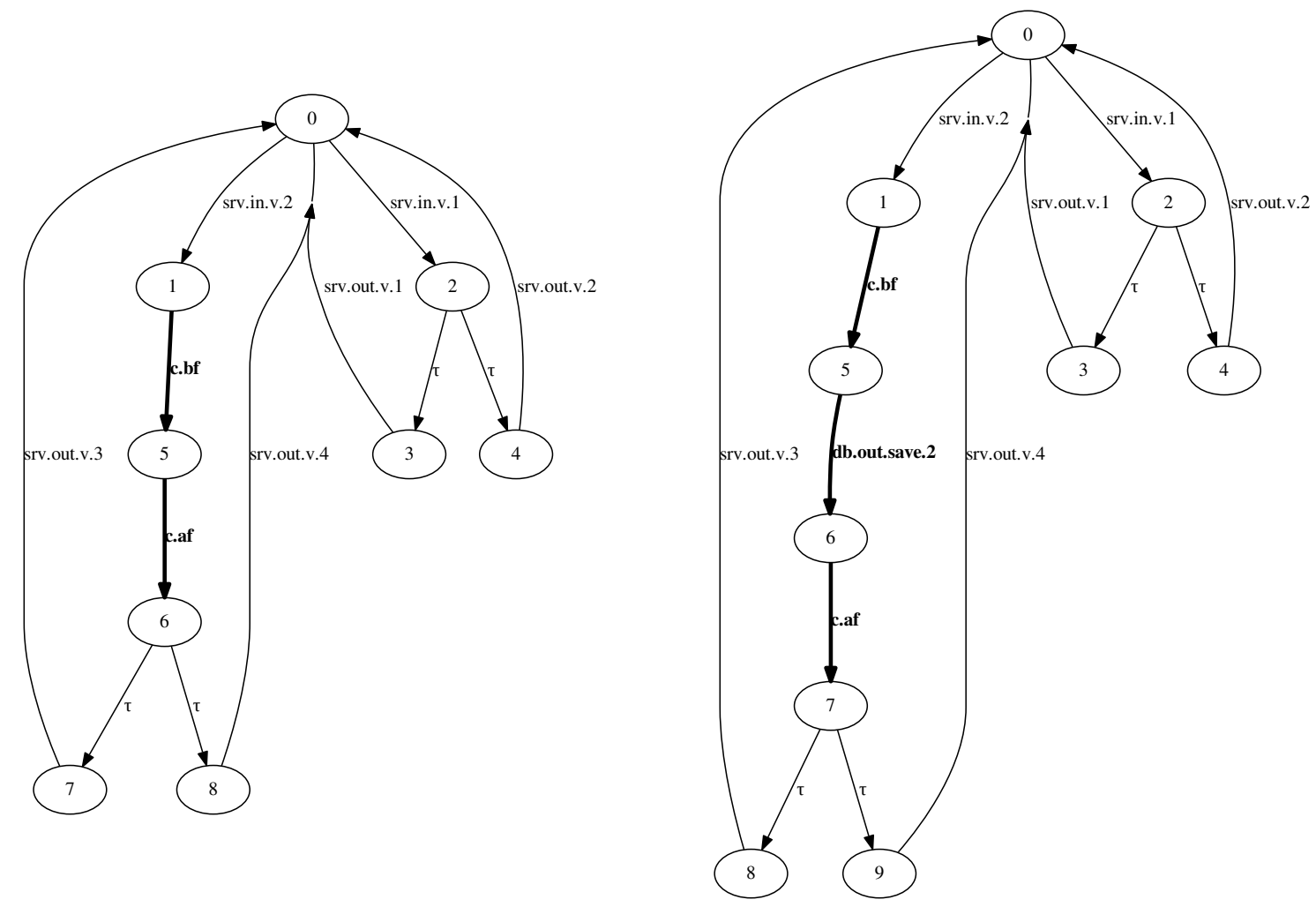

Source: José Dihego

srv.in.v.2, followed by srv.out.v. 3 or srv.out.v.4. Figure 6.2 shows the result component behaviour after weaving the advice $a$ (on the right-hand side) at the join point marked by the pointcut $p$ (on the left-hand side).

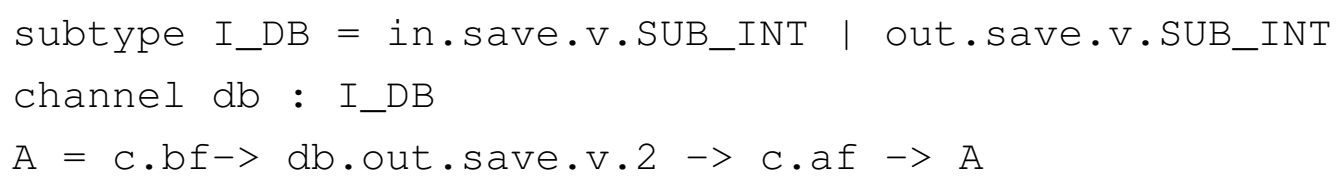

Advices can present different levels of encapsulation, depending on how its functional behaviour $(A \uparrow C)$ is positioned in relation to $c . b f$ and $c . a f$ marking events. We say that $a d v$ is self-contained if $A$, when projected over $C$, is confined between $c . b f$ and $c . a f$ (Definition 6.4). This is the case of $a:\langle\mathrm{A},\{\mathrm{db}\}, \mathrm{c}\rangle$.

Definition 6.4 (Self-contained advice). We say that an advice $a d v:\langle A, C, c\rangle$ is self-contained if and only if:

(i) $(\langle\rangle, \Sigma \backslash\{c . b f\}) \in$ failures $(A)$. Initially, A rejects everything but c.bf;

(ii) $\exists t^{\wedge}\langle$ c.af $\rangle \in \operatorname{traces}(A) \Rightarrow\left(t^{\wedge}\langle c . a f\rangle, \Sigma \backslash\{|c . b f|\}\right) \in$ failures $(A)$. If A performs $c . a f$, then it rejects everything but $c . b f$. 
Finally, we define an aspect as a tuple comprising a pointcut (Definition 6.2) and an advice (Definition 6.3). In Definition 6.5: (i) ensures $p c d$ and $a d v$ agree on the mark events used to signalise join points and (ii) requires the pcd.P and $a d v . A$ alphabets, except for $\{|c|\}$, to be disjoint; it avoids possible interferences between $a d v . A$ and $p c d . P$.

Definition 6.5 (Aspect). An aspect asp : $\langle p c d, a d v\rangle$ is a tuple consisting of an pointcut pcd and an advice $a d v$ such that:

(i) $a d v \cdot c=p c d . c$

(ii) $(\alpha \operatorname{pcd} . P \cap \alpha a d v . A) \subseteq\{|a d v . c|\}$

Recall from Definition 2.3 that a $\mathcal{B R J C}$ component is defined by a behaviour and a set of communication channels by which it interacts with its environment. Aspects act by merging their behaviour (advice) and structure (communication channels) with components. In general, an aspect acts in two ways when being weaved with a component: (behaviourally) it marks join points on component behaviour (maybe in more than one place) and joins its advice behaviour on them; (structurally) the aspect adds the channels used by its advice to the component communication channel set. Therefore, given an aspect $a s p:\langle p c d, a d v\rangle$ and a component $T:\langle\mathcal{B}, \mathcal{R}, \mathcal{J}, \mathcal{C}\rangle$, the task of our ACW (aspect-component weaver) is to search over $\mathcal{B}$ for all join points defined by $p c d$, mark them, weave the $a d v$ 's behaviour at these join points and, finally, extend $\mathcal{C}$ and J by the $a d v$ 's channels and their types, respectively. Definition 6.6 presents the ACW partial (not all aspects and components are weave-able) operator.

Definition 6.6 (Aspect-component weaver). Consider a $\mathcal{B R J \mathcal { C }}$ component $T:\langle\mathcal{B}, \mathcal{R}, \mathcal{J}, \mathcal{C}\rangle$ and an aspect asp : $\langle p c d, a d v\rangle$, such that $a d v:\langle A, C, c\rangle$ and pcd $:\langle P, c\rangle$. We define the ACW (infix) partial operator ${ }_{-} \times{ }_{-}:$As pect $\times \mathcal{B R J C} \rightarrow \mathcal{B R J C}:$

$\operatorname{asp}_{\times}^{\times} T=\left\langle(\mathcal{B}[|s s|] P)[|c|] A, \mathcal{R} \cup R^{\prime}, \mathcal{J} \cup I^{\prime}, \mathcal{C} \cup C\right\rangle$

where

$s s=\alpha P \backslash\{|c|\} \wedge I^{\prime}=\{\operatorname{type}($ ch $) \mid$ ch $\in C\} \wedge R^{\prime}=\{$ ch $\mapsto$ type $($ ch $) \mid$ ch $\in C\}$

provided

$\mathcal{C} \cap C=\emptyset \wedge(\mathcal{B}[|s s|] P) \backslash\{|c|\} \equiv_{\mathrm{F}} \mathcal{B}$

The provided clause above restricts the possible compositions between aspects and components: the act of discovering and marking join points does not change the overall behaviour of the target components ( $\mathcal{B}[|s s|] P \backslash\{|c|\} \equiv_{\mathrm{F}} \mathcal{B}$, where $\equiv_{\mathrm{F}}$ means failure equivalence). Therefore, the mark events $c . b f$ and $c . a f$ do not change, from the environment perspective, the behaviour of $T$; pcd marks at least one join point on $\mathcal{B}$, so $\mathcal{B}[|s s|] P$ deadlocks if both processes cannot agree on the behavioural pattern described by $P$ at least once (the previous failure equivalence 
implies $\mathcal{B}[|s s|] P$ is deadlock free); $(\mathcal{C} \cap C=\emptyset)$ the interaction points of $a d v$ and $T$ are disjoint, so when $A$ is weaved into $T$ there is no clash between their interfaces.

The behaviour of $\operatorname{asp}_{\times}^{\times} T$ is given by $(\mathcal{B}[|s s|] P)[|c|] A$, where $s s=\alpha P \backslash\{|c|\}$. The ACW operator $\times$ acts in two phases over $\mathcal{B}$ : by synchronising it with $P$ on $\alpha P \backslash\{|c|\}$, it marks join points, and then by putting this result in parallel with $A$ on $\{|c|\}$, it inlays $A$ at each join point (between $c . b f$ and $c . a f$ ) marked on $\mathcal{B}$. Concerning the result component structure, the interaction points of $\operatorname{asp}{ }_{\times}^{\times} T$ are $\mathcal{C} \cup C$, the types of $C\left(I^{\prime}\right)$ and $\mathcal{J}$ from its interface, and interaction points and interfaces are related by $\mathcal{R} \cup R^{\prime}$, where $R^{\prime}$ relates $C$ and $I^{\prime}$. Note that in Definition 6.6 we have assumed the behaviour of $a s p \times{ }_{\times}^{\times}$is an I/O process, which is proved by Lemma 6.1 .

Lemma 6.1 ( $\mathcal{B} \mathcal{R J C}$ is closed under ACW). Consider the $\mathcal{B} \mathcal{R J C}$ component $T:\langle\mathcal{B}, \mathcal{R}, \mathcal{J}, \mathcal{C}\rangle$ and the aspect asp : $\langle p c d, a d v\rangle$, such that $p c d:\langle P, c\rangle$ and $a d v:\langle A, C, c\rangle$. If $a s p{ }_{\times}^{\times} T$ is defined, then it is a $\mathcal{B R J C}$ component.

Proof.

\section{Part i (asp $\times$ × $\mathbf{T}$ structure)}

$\left[I^{\prime}=\{\right.$ type $($ ch $) \mid$ ch $\in C\}, R^{\prime}=\{$ ch $\mapsto$ type $($ ch $) \mid c h \in C\}$, Definition 6.6 $]$

$\mathcal{R}_{a s p \times T}^{\times}=\mathcal{R} \cup R^{\prime} \wedge \mathcal{J}_{a s p \times}^{\times} T=\mathcal{J} \cup I^{\prime} \wedge \mathcal{C}_{a s p \times T}=\mathcal{C} \cup C$

$\Rightarrow \mathcal{R}$ is a total function between $\mathcal{C}$ and $\mathcal{J} \wedge \mathcal{R}^{\prime}$ is a total function between $C$ and $I^{\prime}$

$\Rightarrow[\mathrm{C} \cap C=\emptyset]$

$\mathcal{R}_{a s p \times}^{\times}$is a total function between $\mathcal{C}_{a s p \times}{ }_{\times}$and $\mathcal{J}_{a s p \times}{ }_{\times} T$

\section{Part ii (asp $\stackrel{\times}{\times} \mathbf{T}$ behaviour)}

[Definition 6.6, ss $=\alpha P \backslash\{|c|\}]$

$\mathcal{B}_{\text {asp } \times T}=(\mathcal{B}[|s s|] P)[|c|] A \wedge(\mathcal{B}[|s s|] P) \backslash\{|c|\} \equiv_{\mathrm{F}} \mathcal{B}$

$\Rightarrow$ [Definition 2.4, $\equiv_{\mathrm{F}}$ semantics (ROSCOE, 1998)]

$(\mathcal{B}[|s s|] P) \backslash\{|c|\}$ is an $\mathrm{I} / \mathrm{O}$ process

$\Rightarrow[c . b f \in$ inputs , hiding semantics (ROSCOE, 1998),(i - iii - iv) of Definition 6.2)]

$\mathcal{B}[|s s|] P$ is an $\mathrm{I} / \mathrm{O}$ process

$\Rightarrow$ [Advice (Definition 6.3]

$(\mathcal{B}[|s s|] P)$ and $(A \backslash\{|c|\})$ are I/O processes

$\Rightarrow[c . b f \in$ inputs $\wedge$ input determinism (Definition 2.4) $]$

$(\mathcal{B}[|s s|] P)$ and $A$ are $\mathrm{I} / \mathrm{O}$ processes

$\Rightarrow[(\alpha(\mathcal{B}[|s s|] P) \cap \alpha A)=\{|c|\}$, parallelism semantics (ROSCOE, 1998), I/O process (Definition 2.4) $]$ $(\mathcal{B}[|s s|] P)[|c|] A$ is an $\mathrm{I} / \mathrm{O}$ process

By Definition 6.5 the aspect $L O G:\langle p, a\rangle$ modularises the logging feature we have developed alongside our aspect theory and, by Definition 6.6, the result of weaving $L O G$ with 
Figure 6.3: Weaving $L O G$ and $C t r_{\mathrm{SRV}}$

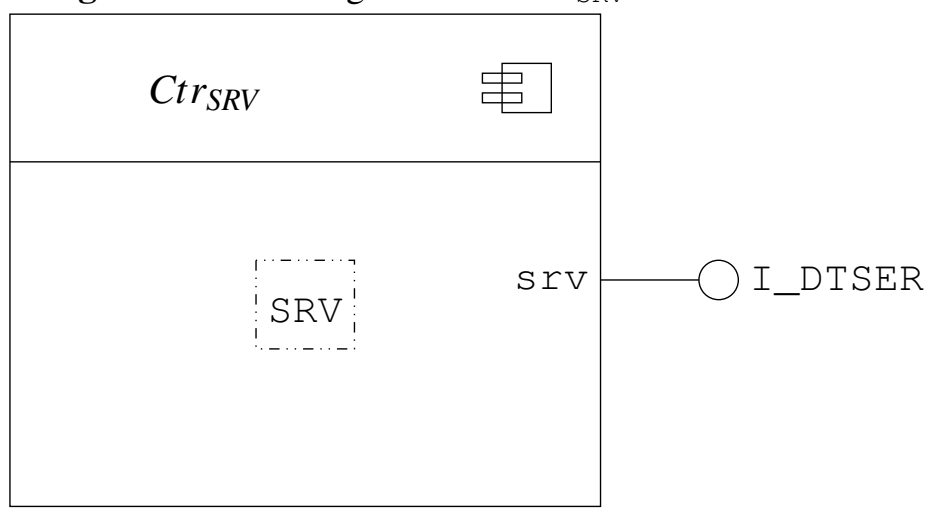

$\Downarrow L O G$ weaving

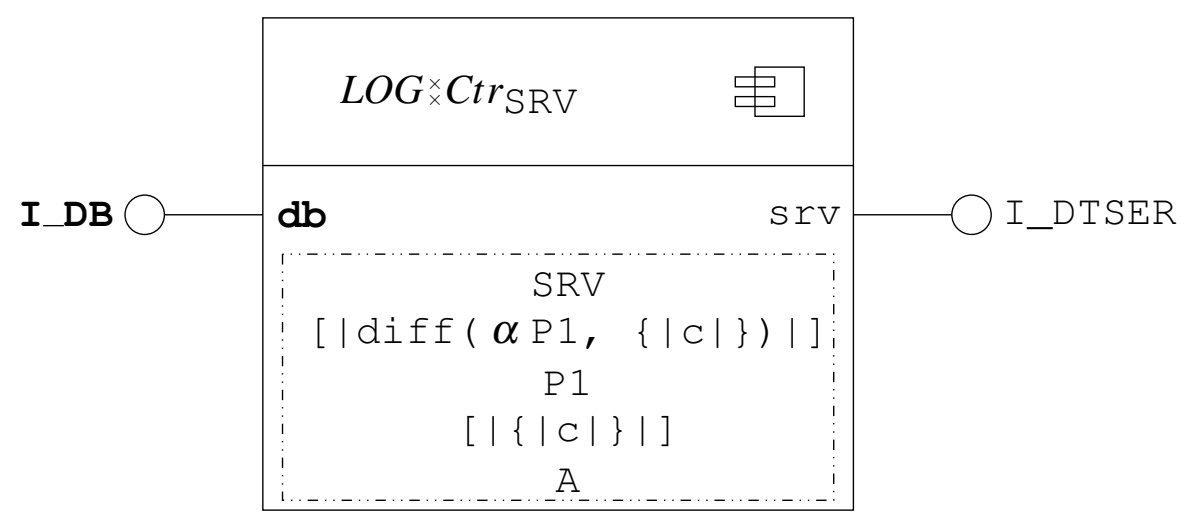

Source: José Dihego

$C t r_{\mathrm{SRV}}$ is the component $L O G_{\times}^{\times} \mathrm{Ctr}_{\mathrm{SRV}}$ bellow. We emphasise that it is possible to reuse both $p$ and $a$ with other pointcuts and advices to capture new aspects, given the loose coupling between them. Figure 6.3 summarises the effect of weaving $L O G$ with $C t r_{\mathrm{SRV}}$.

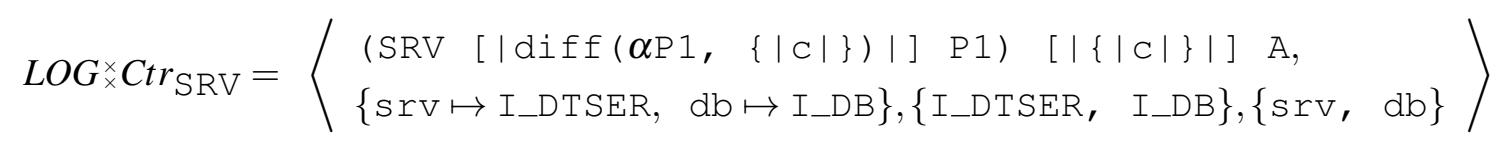

\section{Safely evolving BRIC components using aspects}

In this section we discuss how AOD can be used to safely evolve $\mathcal{B} \mathcal{R J C}$ specifications. In Chapter 4, we develop two $\mathcal{B R J C}$ extension relations based on the concept of behavioural convergence (and its extended version), which fulfil the substitutability principle (WEGNER; ZDONIK, 1988): a component extension should be usable wherever the original component was expected, without any other component, acting as a client, being able to tell the difference. In this section we prove that a(n) (extended) convergent component can be concisely and elegantly generated from an original component by using aspects in the style defined in the previous section; 
so the original and the (aspect oriented) evolved component satisfy the $\mathcal{B R J C}$ substitutability principle.

Convergence is a behavioural relation between I/O processes. An I/O process $\mathcal{B}^{\prime}$ is extended convergent to $\mathcal{B}$ ( $\mathcal{B}^{\prime}$ io_ecvg $\mathcal{B}$ ), according to Definition 4.4 , if in each converging point of their execution it can offer more or equal inputs but is restricted to offer less or equal outputs. It can also offer any other event after a new input and before converging to its original behaviour, adding more implementation details. A convergent point represents a state reachable by both the original and the convergent process when doing two convergent sequences of events; these sequences differ only because the convergent process is allowed to do extra new-in-contextinputs (inputs not allowed by the original process at that point) in converging points followed by any event not allowed by the original process.

Consider the I/O processes SRV (on the left-hand side of Figure 6.1) and SRV' (on the right-hand side of Figure 6.2). Based on Definition 4.4, we have that SRV' is extended convergent to SRV (SRV' io_ecvg SRV). To explain why this is the case, let $\left(t^{\prime}, X\right)$ and $(t, Y)$ be failures of SRV' and SRV, respectively. Then by a non-exhaustive analysis:

- If $\left(t^{\prime}, X\right)=(\langle\operatorname{srv} \cdot i n . v .2\rangle, \Sigma \backslash\{c . b f\})$, then we know that $t^{\prime}$ ecvg $t$, for $t=\langle$ srv.in.v. 2$\rangle$. The failure after $t$ is $(t, Y)=(\langle$ srv.in.v. $\rangle\rangle, \Sigma \backslash\{$ srv.out.v. 3$\})$, and $Y \cap$ inputs $\supset X \cap$ inputs and $Y \cap$ outputs $\subset X \cap$ outputs;

- If $\left(t^{\prime}, X\right)=(\langle$ srv.in.v. $2, c . b f\rangle, \Sigma \backslash\{$ db.out.save. 2$\})$, then we know $t^{\prime}$ ecvg $t$, for $t=$ $\langle$ srv.in.v. 2$\rangle$ because $c . b f \in$ inputs and $c . b f \in \operatorname{in}(\mathrm{SRV}, t)$. The failure after $t$ is $(t, Y)=$

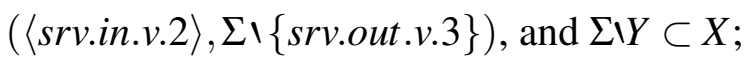

These pairs satisfy the extended convergence relation (Definition 4.4). In fact, for any member of $\mathcal{F}\left(S R V^{\prime}\right)$ we can find at least one convergence related failure in $\mathcal{F}(\mathrm{SRV})$. Indeed, we have proved this fact in FDR3 by checking the following assertion:

Tester_ecvg (SRV_serial) [F= SRV', where SRV_serial stands for the serialised version of SRV (see Section 4.3).

In the beginning of this section, we emphasised that convergence is a behavioural relation between I/O processes, not between components. In Chapters 3 and 4 we have defined refinement and inheritance relations for $\mathcal{B R J C}$ (Definition 4.7), which consider components structure (channels and interfaces) and behaviour (I/O processes).

A component inherits (by convergence) from another if: structurally, the inherited component preserves at least its parent's channels and their types. Regarding behaviour, they are related by convergence (or its extended version). Additionally, inheritance guarantees, for the purpose of substitutability, that the inherited component only refines the behaviour exhibited by common channels (default channel congruence, Definition 4.5) or that additional inputs over common channels are not exercised by any possible client of its parent (input channel congruence, Definition 4.6). 
We already know that SRV' io_ecvg SRV and $L O G_{\times}^{\times} C^{\circ} r_{\mathrm{SRV}}$ channels extends those of $C t r_{\mathrm{SRV}}$ (Figure 6.3). Additionally, SRV' is default channel congruent w.r.t SRV (on their common channels, Definition 4.5). Therefore, accordingly to Definition 4.7 we have:

$$
C t r_{\mathrm{SRV}} \leftarrow_{e c v g} L O G_{\times}^{\times} \operatorname{Ctr}_{\mathrm{SRV}}
$$

In fact, this relation between aspects and inheritance always holds as proved by Theorem 6.1.

Theorem 6.1 (aspects and inheritance). Let $T$ and asp stand for a component and an aspect, respectively. If $\operatorname{asp}{ }_{\times}^{\times} T$ is defined, then $T \leftarrow e c v g$ as ${ }_{\times}^{\times} T$, provided asp.adv is self-contained (see Definition 6.4).

Proof.

$$
\begin{aligned}
& T^{\prime} \\
& =\left[\begin{array}{l}
\text { Definition 6.6, } T:\langle\mathcal{B}, \mathcal{R}, \mathcal{J}, \mathcal{C}\rangle, \text { asp }:\langle\langle P, c\rangle,\langle A, C, c\rangle\rangle, \\
T^{\prime}=\operatorname{asp}{ }_{\times}^{\times} T, I^{\prime}=\{\operatorname{type}(\operatorname{ch}) \mid \operatorname{ch} \in C\}, R^{\prime}=\{c h \mapsto \text { type }(\text { ch }) \mid c h \in C\}
\end{array}\right] \\
& \left\langle(\mathcal{B}[|\alpha P \backslash\{|c|\}|] P)[|c|] A, \mathcal{R} \cup R^{\prime}, \mathcal{J} \cup I^{\prime}, \mathcal{C} \cup C\right\rangle \\
& \Rightarrow \text { [Definition 6.6, } \mathcal{C} \cap C=\emptyset \text {, set theory] } \\
& \text { (i) } \mathcal{R}_{T} \subseteq \mathcal{R}_{T^{\prime}} \\
& \Rightarrow\left[\begin{array}{l}
\text { Definitions 6.5, 6.6, 4.7 and 4.5, alphabetised parallelism semantics (Section 2.1), } \\
(\mathcal{B}[|\alpha P \backslash\{|c|\}|] P) \backslash\{|c|\} \equiv_{\mathrm{F}} \mathcal{B}, \alpha P \backslash\{|c|\} \subseteq \alpha \mathcal{B}, \alpha A \cap \alpha \mathcal{B}=\emptyset, \alpha A \cap \alpha P=\{|c|\}
\end{array}\right] \\
& \text { (ii) } \forall c: \mathcal{C}_{T} \bullet \mathcal{B}_{T^{\prime}} \text { def-cong }{ }_{\{c\}} \mathcal{B}_{T} \\
& \Rightarrow\left[\begin{array}{l}
\text { (iii - iv) of Definition 6.2, Definition 4.4, Definition 6.1 (c.bf } \in \text { inputs), parallelism } \\
\text { semantics, }(\mathcal{B}[|\alpha P \backslash\{|c|\}|] P) \backslash\{|c|\} \equiv_{\mathrm{F}} \mathcal{B}, \alpha P \backslash\{|c|\} \subseteq \alpha \mathcal{B}, \quad\{|c|\} \cap \alpha \mathcal{B}=\emptyset
\end{array}\right] \\
& \exists t^{\wedge}\langle c . b f\rangle \in \mathcal{T}(P) \Rightarrow\left(t^{\wedge}\langle c . b f\rangle, \Sigma \backslash\{c . a f\}\right) \in \mathcal{F}(P) \wedge(t, \Sigma \backslash\{c . b f\}) \in \mathcal{F}(P) \wedge \\
& \forall\left(t^{\prime}, X\right) \in \mathcal{F}(\mathcal{B}[|\alpha P \backslash\{|c|\}|] P), \exists(t, Y) \in \mathcal{F}(\mathcal{B}) \bullet t^{\prime} \operatorname{ecvg} t \wedge \\
& \left(\begin{array}{c}
Y \cap \text { inputs } \supseteq X \cap \text { inputs } \wedge \\
Y \cap \text { outputs } \subseteq X \cap \text { outputs }
\end{array}\right) \\
& \Rightarrow \mathcal{B}[|\alpha P \backslash\{|c|\}|] P \text { io_ecvg } \mathcal{B} \\
& \Rightarrow \text { [Definition 6.4] } \\
& \exists t^{\wedge}\langle c . a f\rangle \in \mathcal{T}(A) \Rightarrow\left(t^{\wedge}\langle c . a f\rangle, \Sigma \backslash\{|c . b f|\}\right) \in \mathcal{F}(A) \wedge(\langle\rangle, \Sigma \backslash\{c . b f\}) \in \mathcal{F}(A) \\
& \Rightarrow[\alpha A \cap \alpha \mathcal{B}[|\alpha P \backslash\{|c|\}|] P=\emptyset, \text { (i - iv - v) Definition 6.3] } \\
& (\mathcal{B}[|\alpha P \backslash\{|c|\}|] P)[|c|] A \text { io_ecvg } \mathcal{B}[|\alpha P \backslash\{|c|\}|] P \\
& \Rightarrow\left[(\mathcal{B}[|\alpha P \backslash\{|c|\}|] P) \backslash\{|c|\} \equiv_{\mathrm{F}} \mathcal{B}\right. \text {, Definition 6.6] } \\
& \text { (iii) }(\mathcal{B}[|\alpha P \backslash\{|c|\}|] P)[|c|] A \text { io_ecvg } \mathcal{B} \\
& \Rightarrow[(i), \quad(i i), \quad(i i i), \text { Definition 4.7] } \\
& \mathbf{T} \leftarrow_{\operatorname{ecvg}} \operatorname{asp} \times \mathbf{T}
\end{aligned}
$$


Considering the substitutability principle for $\mathcal{B R J C}$ (with focus on deadlock freedom, but not limited to it) as stated in Theorem 4.2 (Chapter 4) and Theorem 6.1 we enunciate the relevant Corollary 6.1.

Corollary 6.1 (Aspects preserve substitutability). Let $T$ and as $p$ stand for a component and an aspect, respectively. Consider $S[T]$ a deadlock free component contract, where $T$ is a basic component contract that was composed within $S$ using one of the $\mathcal{B R J C}$ composition rules. If $\operatorname{asp}{ }_{\times}^{\times} T$ is defined, then $S[a s p \times T]$ is deadlock free.

The above corollary follows direct from Theorems 6.1 and 4.2. As far as the authors are aware, this is the first time a relation between component inheritance and aspects-oriented design is established for a formal approach to CB-MDD. It offers two ways to evolve component specifications that, although distinct in concept, are related by their implementation mechanisms. It is important to make clear that aspects are designed to cope with crosscutting concerns that scatter through families of components by enlarging them, where inheritance is exclusively designed to create such families. It is fair to say that aspects operate horizontally and inheritance vertically over component family trees.

As a final remark, we highlight that our aspect-oriented design for $\mathcal{B R J C}$ is driven by the need to act over and produce deadlock-free component-based specifications. Therefore, more simpler aspect definitions, for example by considering non-deterministic join point marking processes, are not addressed in this work because they can, potentially, produce components outside the model, which can, perfectly, be accepted in less restrictive CB-MDD approaches such as UML-RT (SELIC; RUMBAUGH, 1998) or Rcos (JIFENG; LI; LIU, 2005).

\section{Case study}

In Chapter 5, we have developed the autonomous healthcare robot component $C \operatorname{tr}_{H C \_B O T}$ to monitor and medicate patients; it is also able to contact the relevant individuals or systems in case of emergency. It receives data from a number of sensors and actuates by injecting intravenous drugs and/or by calling the emergency medical services and the patient's relatives or neighbours.

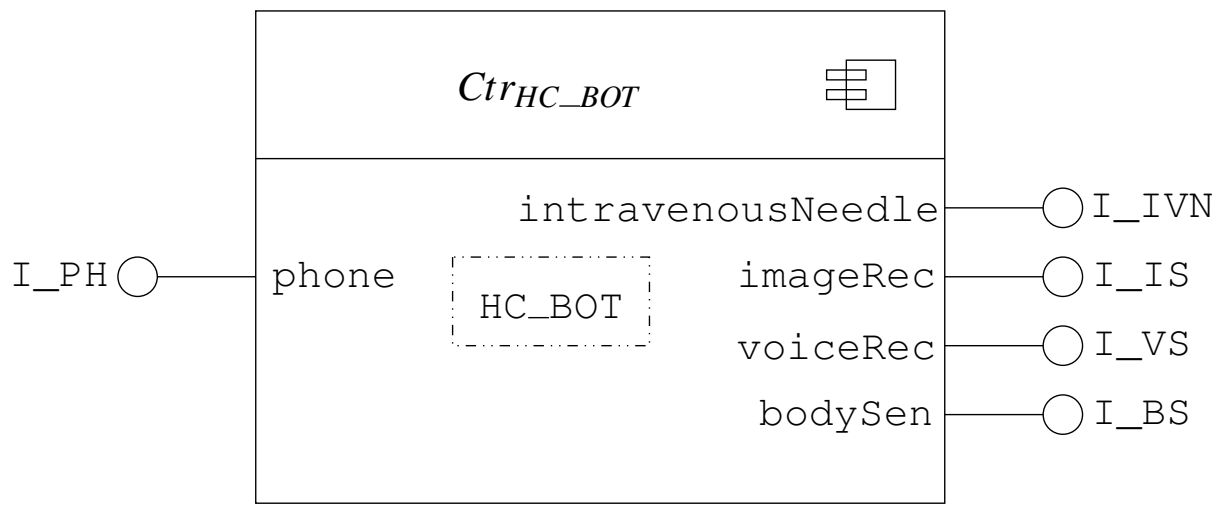


The robot $C \operatorname{tr}_{\text {HC_BOT }}$ is able to diagnose and select the appropriate drug to be administered. Nevertheless, there is no criteria to define an appropriate drug dose given the seriousness of the patient condition. This enhancement is defined by the contract $C_{t} r_{H C \_B O T \_A C C}$. It has a better (more deterministic) decision-making mechanism on the drug dose to be administered to the patient it monitors, which takes into account the body temperature and blood glucose values.

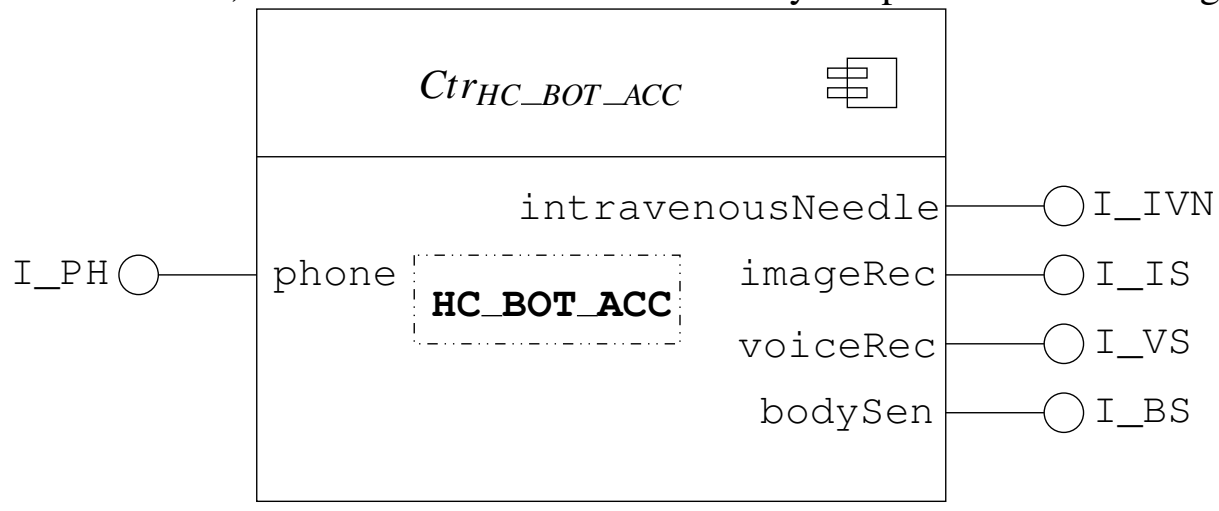

A major improvement was achieved with the component $C t r_{H C \_B O T \_T K}$; it extends (inherits from) $C t r_{H C \_B O T \_A C C}$ with the addition of a talk module, which allows this robot to ask patients about their symptoms and thus better help them.

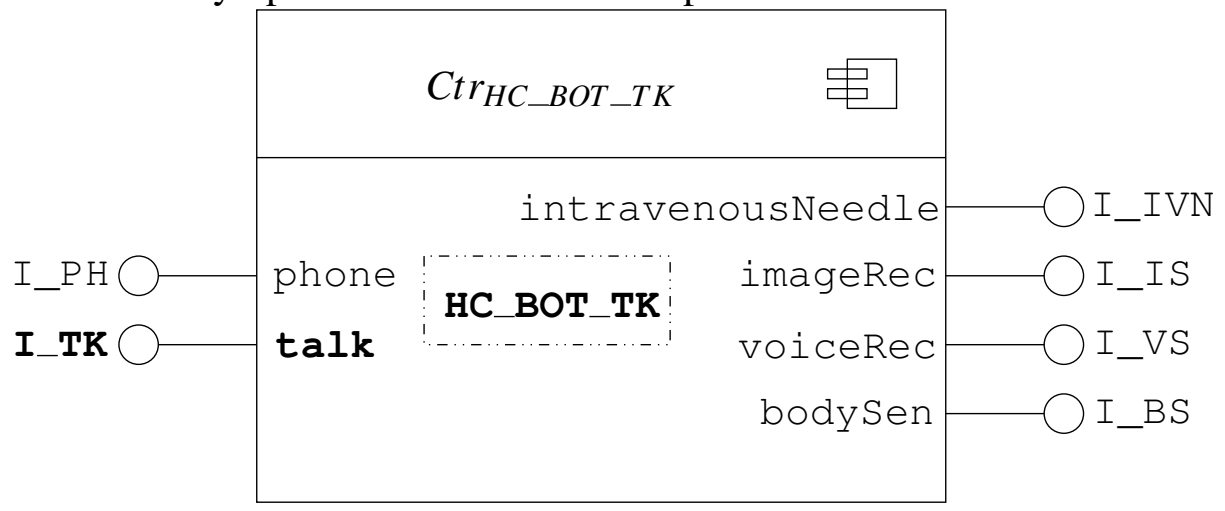

According to Definitions 4.7 and 3.1, we have that (see Figure 5.4):

$$
\begin{gathered}
C t r_{H C \_B O T} \sqsubseteq_{\mathcal{B}} C t r_{H C \_B O T \_A C C} \\
C t r_{H C \_B O T \_A C C} \longleftarrow_{e c v g} C t r_{H C \_B O T \_T K}
\end{gathered}
$$

We can have many kinds of robots attached to a patient, sharing an extremely critical resource, his/her intravenous access, a common point where drugs can be injected without doctors supervision. In life threatening scenarios, doctors can be compelled to intervene, but they will need to know which robot injected which drug and in which dose, so they can proceed with the appropriate intervention.

This is a classic problem, which has a well-known solution: logging. Therefore, we must, originally, redefine each component in our specification to add the logging feature. As new communication channels must be used to implement the logging functionality, we need to update component interfaces to refer to these new channels as well as their types. If we have many types of robots, we must face an exhaustive and error prone task in updating each 
of them and, worst, it must be done each time we need to change the logging and/or robots specifications. Thanks to our AOD approach to CB-MDD, we can solve this problem in a more modular and maintainable way by defining a logging aspect, which can enhance the $\mathrm{Ctr}_{\text {HC_BOT }}$, $C t r_{H C \_B O T \_A C C}$ and $C t r_{H C \_B O T \_T K}$ components with this feature.

We identify each robot with a unique identifier, a member of BOT_ID, whose elements range from one to TOP (a natural number). First we define a check mark channel and a pointcut to capture the condition we are interested on: a robot has administered a drug. We define the tags $\mathrm{b} f$ and $a f$, which are used by the check mark channel drug_mark. It communicates $\{\mathrm{b} f, \mathrm{a} f\}$ alongside the injected drug DRUG and its respective dose DD. The process DET_DRUD synchronises on any access to the intravenous needle (intravenousNeedle. out.administer. $\mathrm{dg} \cdot \mathrm{dose}$ ) and marks it as a join point by performing the events drug_mark.bf.dg.dose and drug_mark.af.dg.dose sequentially.

channel drug_mark : $\{\mathrm{bf}, \mathrm{af}\} . \mathrm{DRUG} . \mathrm{DD}$

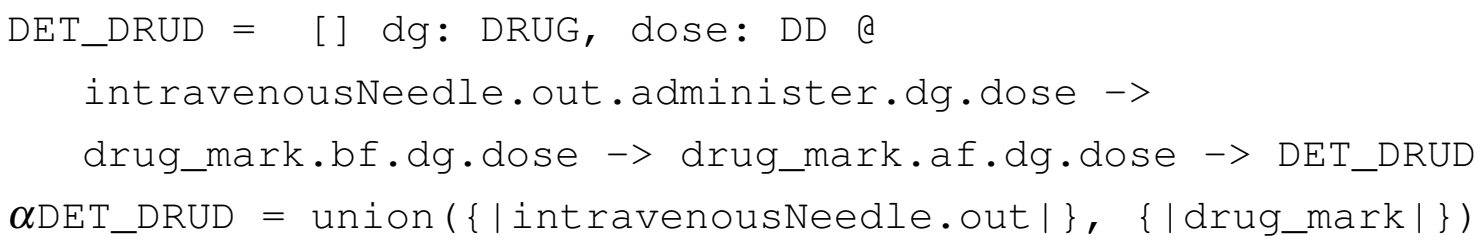

With the CSP process DET_DRUD and the check mark channel drug_mark, we define the pointcut $P D:\langle$ DET_DRUD, drug_mark $\rangle$, which can detect and mark every time a robot uses the patient intravenous access.

We want to log triples: which robot has administered, which drug, in which quantity. The channel drug_log will be used by our advice to log the fact that some robot (BOT_ID) has administered a dose of a given drug (DRUG.DD) to its target patient. The advice behaviour is given by the I/O process LOG_DRUG $(x)$; it synchronises on the check mark event drug_mark.bf.dg.dose, then logs the fact that the robot $x$ has injected the amount dose of the drug dg (drug_log.out.log.x.dg.dose). After, it agrees on drug_mark.af.dg.dose (the join point end mark) and goes to its initial state.

channel drug_log: in.log.BOT_ID.DRUG.DD|out.log.BOT_ID.DRUG.DD

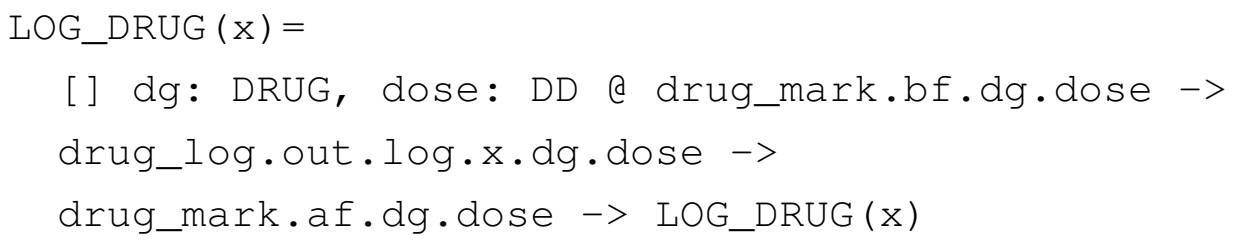

With the I/O process LOG_DRUG (x), the check mark channel drug_mark and the communication channel drug_mark we define an advice to search and modify each join point marked by $P D: A D$ : 〈LOG_DRUG $(\mathrm{x}),\{$ drug_log $\}$, drug_mark $\rangle$. Finally we define our intended logging drug aspect as $L O G \_D:\langle P D, A D\rangle$. It can be weaved (Definition 6.6) into 
$C t r_{H C \_B O T}, C t r_{H C \_B O T \_A C C}$ and $C t r_{H C \_B O T \_T K}$, resulting, respectively (from top to bottom), in the components depicted in Figure 6.4.

By Theorem 6.1, we can establish the following results, which present AOD as a proper way to introduce inheritance in component-based specifications.

$$
\begin{aligned}
& C t r_{H C \_B O T} \leftarrow_{e c v g} L O G \_D{ }_{\times}^{\times} C t r_{H C \_B O T} \\
& C t r_{H C \_B O T \_A C C} \leftarrow e c v g L O G \_D \times C t r_{H C \_B O T \_A C C} \\
& C t r_{H C \_B O T \_T K} \triangleleft e c v g\left[O G \_D \times C t r_{H C \_B O T \_T K}\right.
\end{aligned}
$$

Another important result comes from the monotonicity of the ACW operator (Definition 6.6) over component inheritance (Definition 4.7) and refinement (Definition 3.1).

$$
\begin{aligned}
& L O G \_D{ }_{\times}^{\times} C t r_{H C \_B O T} \sqsubseteq_{\mathcal{B}} L O G \_D{ }_{\times}^{\times} C t r_{H C \_B O T \_A C C} \\
& L O G \_D \times C t r_{H C \_B O T \_A C C} \leftarrow e c v g L O G \_D \times C t r_{H C \_B O T \_T K}
\end{aligned}
$$

To conclude this case study we present the following results. They come from the transitivity property of inheritance, the monotonicity of ACW over it and, by the fact that $\sqsubseteq_{\mathcal{B}} \subset \triangleleft_{\text {ecvg }}$ (see Figure 4.4):

$$
\begin{aligned}
& C t r_{H C \_B O T} \leftarrow e c v g L O G \_D \times C t r H C \_B O T \_T K \\
& L O G \_D \times C t r_{H C \_B O T} \leftarrow e c v g L O G \_D \times C t r_{H C \_B O T \_T K}
\end{aligned}
$$


Figure 6.4: ACW preserves the healthcare autonomous system hierarchy

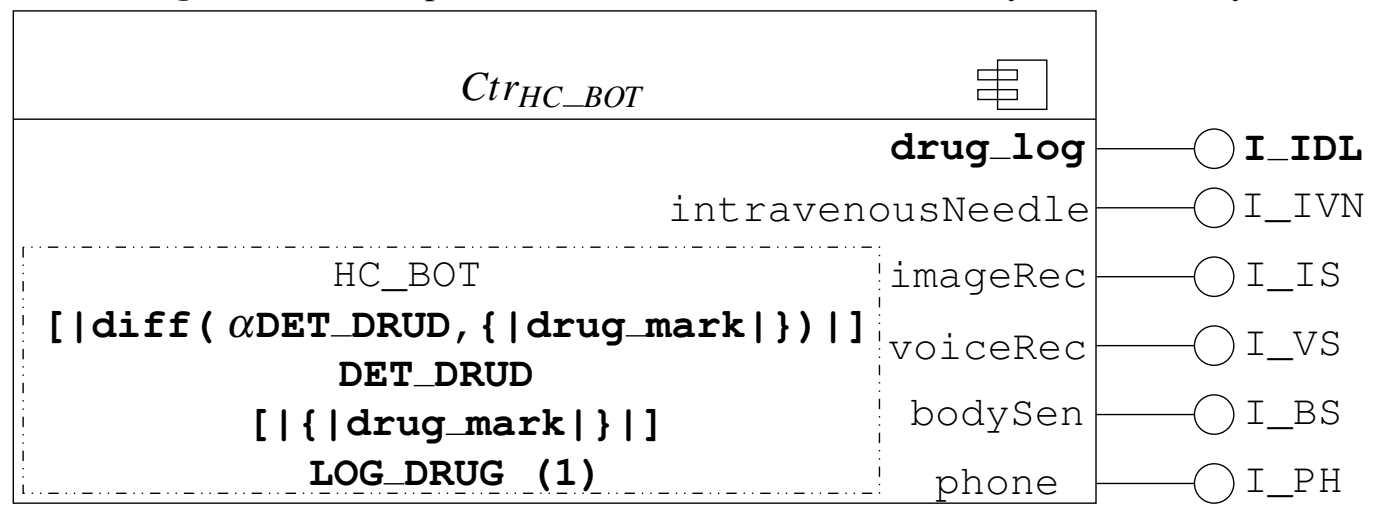

$\sqsubseteq \mathcal{B}$
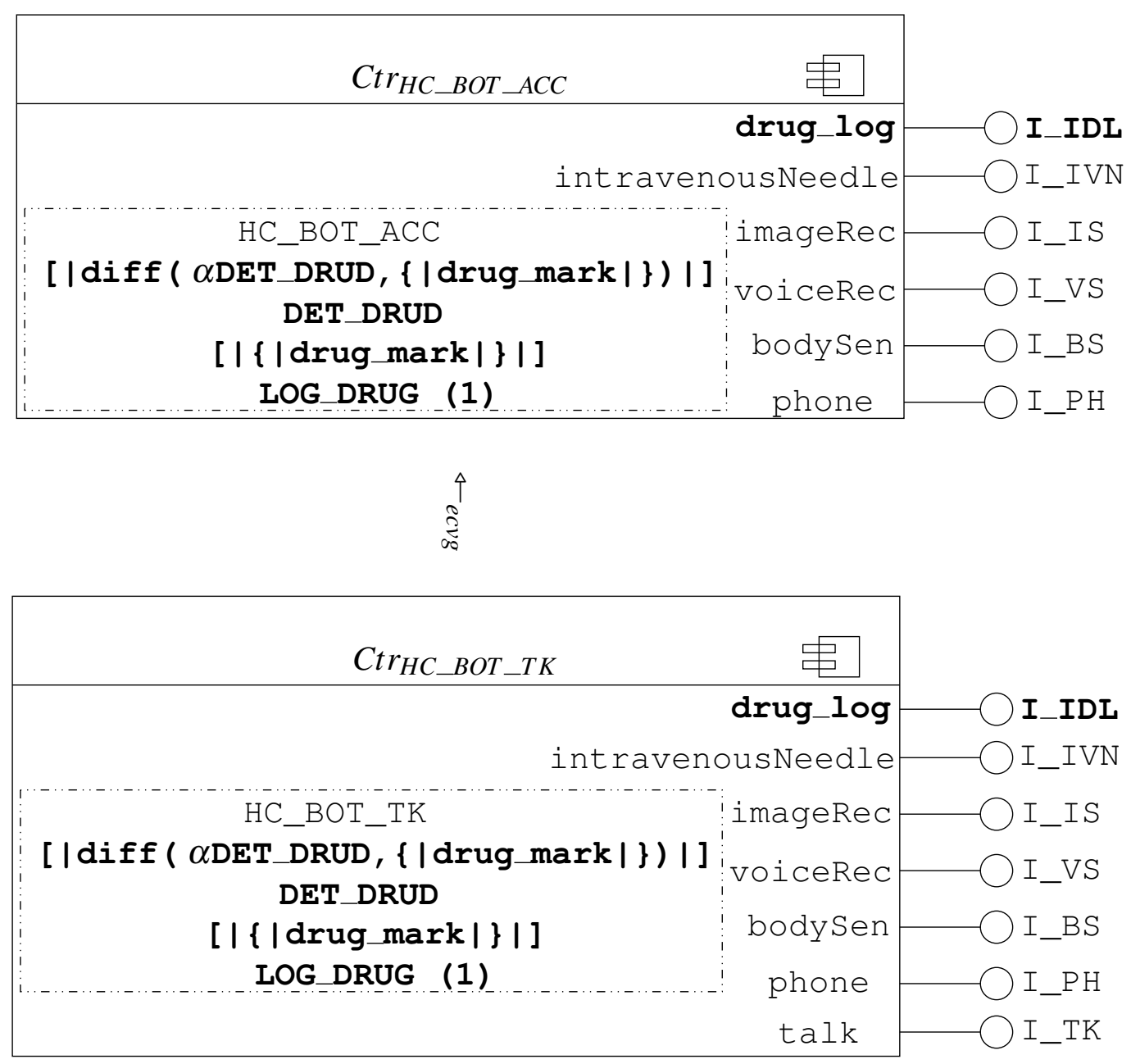

Source: José Dihego 


\section{7}

\section{Conclusions, related and future work}

In this chapter we present the main contributions of our work. Furthermore, we discuss related work on component refinement and inheritance. We conclude with the challenges and opportunities opened by this work.

\section{Contributions}

A crucial aspect of the specification/development languages is how they support system evolution, which typically involves enhancing existing services, meeting non-functional requirements or delivering new functionalities. This is also true for the approaches to component-based model driven development (CB-MDD) (SZYPERSKI, 1998). The BRJC component model includes important features like the formalisation of the CB-MDD core concepts and a set of composition rules that ensure deadlock freedom by construction. Nevertheless, it has not addressed how component models can evolve, through refinement and inheritance, for instance. This is what this work is about and the following summarises our main achievements:

BRIC semantics. We have defined a congruent semantics for $\mathcal{B} \mathcal{R J C}$ that considers component structure and behaviour. This first step makes it possible to understand the precise meaning of a component, and is the basis to define component refinement and inheritance.

BRIC refinement. Having a formal semantics, we have defined a refinement relation for the $\mathcal{B R J C}$ component model. It guarantees the relevant properties required by the $\mathcal{B R J C}$ component compositions, fulfilling the substitutability principle (a refinement should be usable wherever its abstraction is expected, without a client being able to tell the difference). We have proved that the $\mathcal{B} \mathcal{R J C}$ operators interleave, communication, feedback and reflexive compositions are monotonic with respect to refinement. We also have demonstrated it is a preorder relation and, moreover, preserves deadlock freedom; this last result was proved as a consequence of the results about failures refinement: it preserves I/O confluence, protocol conjugation, strong protocol compatibility, finite output property, decoupled channels and self-injection compatibility, which are the relevant properties assumed by the $\mathcal{B R J C}$ composition rules. 
BRIC inheritance. We have defined two inheritance relation for $\mathcal{B R J \mathcal { C }}$, both based on a novel concept called behavioural convergence. It captures the idea that components can evolve by accepting new inputs or establishing a communication session after these inputs, but then they must converge to the predicted behaviour exhibited by its abstraction.

Our definition of inheritance deals with component structural and behavioural aspects and guarantees substitutability, in the composition rules, preserving deadlock freedom. Therefore a component of a model can evolve by the reduction of non-determinism (refinement) or by the extension of functionality (inheritance) and still preserving, in the entire model, deadlock freedom and protocol compatibility. The protocol compatibility property is a direct consequence of our refinement relation, which relies upon I/O process failures refinement. The same result for inheritance, which is defined upon convergence, needs additional conditions enforced by default and input channel congruence, a concept drawn in this work to guarantee that the behaviour over a component channel is not distorted by an inherited component.

We have two forms of convergence and, consequently, two inheritance relations upon them. We have proved that one is a generalisation of the other. Indeed, we have proved our relations form a hierarchy, where component refinement is the strongest relation between $\mathcal{B R J \mathcal { C }}$ components. It is, in fact, the strongest form of inheritance and, additionally, inheritance by convergence is the most strict form of inheritance by extended convergence, as their names suggest.

We have developed an automated strategy for verifying convergence as refinement assertions using FDR3 (Failures-Divergence Refinement) (GIBSON-ROBINSON et al., 2014). We have systematised the construction of a least upper bound process (under the failures refinement relation), for which all convergent processes are stable-failure refinements. Based on this result, we have converted an assertion about convergence (and extended convergence) into a refinement verification, which can be carried out by FDR3. We have based the construction of this lower bound process on a serialised representation for $\mathrm{I} / \mathrm{O}$ processes, which is a structured sequence that allows backtracking traversal, a relevant aspect to build this process.

BRIC aspect-oriented modelling We defined a formal approach to introduce aspectoriented modelling into component-based specifications, where preservation of some properties is guaranteed by construction. We developed for the $\mathcal{B R J C}$ component model a proper way to characterise pointcuts, advices and aspects. We proved aspects preserve a conformance notion, given in terms of the substitutability principle (WEGNER; ZDONIK, 1988). We also established a connection between component inheritance and aspects, presenting them as interchangeable ways to safely extend component specifications.

We brought the recognised aspects benefits (crosscutting concerns modularisation, reuse and maintainability (KICZALES et al., 1997)) to a trustworthy component-base model, where behaviour properties can be compositionally verified (RAMOS; SAMPAIO; MOTA, 2009). We have not addressed the entire aspects theory, but created a formal basis to its adoption in CB-MDD. 
To illustrate how the design of component-based specifications can benefit from an aspect theory, we developed a case study of an autonomous healthcare system, which evolve by the addition of new functionalities via inheritance and by the modularisation of its crosscutting concerns in a reusable and maintainable manner with aspects. We show how these concepts can be put together benefiting design, clarity and maintainability.

Case studies. We concluded with three case studies that exemplify the use of inheritance to improve an autonomous healthcare robot, a bank system and a P2P network, by decreasing the level of abstraction and increasing determinism. At first, we have modelled an autonomous healthcare robot that monitors and proper medicates patients, being able to communicate with the relevant individuals or systems about the incidents or emergencies that might occur. We refine the initial model by improving the mechanism used to decide over the drug's dose to be administered to the patient. Then, through inheritance, we add a communication module to the reboot, which makes it able to talk with the patient and, by using his answers, it is able to take better decisions about his state of health.

The second case study was a bank system. Initially we have modelled an ATM process that interacts with customers to provide services like withdraw, deposit, transfer and check balance, available after successful login. This ATM handles costumer requests in a non-deterministic manner, as it has no ability to communicate with its bank server. Therefore, we developed, by the use of inheritance an enhanced ATM, which has the ability to handle user requests to the bank server and then present a more concrete behaviour.

In the third case study we develop a P2P network based on the Gnutella (CLIP2, 2001) protocol: a well known implementation of a completely decentralised, flattered P2P network, designed primarily for file sharing. By extending this network via inheritance we achieve traffic reduction by potentially increasing the connection between nodes.

In summary, we have developed in this work a novel concept called behavioural convergence for specifications that distinguish inputs from outputs. Based on that we have developed refinement and inheritance relations for an approach to CB-MDD, where we consider structural and behavioural aspects. We incorporated these relations in a set of composition rules that guarantee deadlock freedom by construction. We also have developed an aspect-oriented modelling approach to capture and model orthogonal aspects that spread though system specifications; by presenting aspects as mechanism for creating convergent behaviours, we have related aspects and inheritance. Putting our results and those of (RAMOS, 2011) together, we can fairly affirm that $\mathcal{B R J C}$ is a fully formal approach for component-based model driven development. We have defined a semantics, a refinement and inheritance relations for components, not only for those expressed in $\mathcal{B R J C}$, but for any other model which differentiates inputs form outputs as (BERTRAND et al., 2011) does. No matter the representation choice, convergence is a broader concept by itself and can be applied in other formal approaches to CB-MDD. 


\section{Related work}

CB-MDD relies on the interface, contract, connector and component concepts. Moreover, a formal approach to CB-MDD must specify how components can be assembled into more complex/functional ones, how they can be refined and, ultimately, how evolve them into more specialised or functional ones. Several works have tried to develop a formal foundation for CBMDD: (RAMOS, 2011), (LIU; MORISSET; STOLZ, 2009), (JIFENG; LI; LIU, 2005), (WANG; WANG; ZHAN, 2009), (JIFENG; LI; LIU, 2006), (CHEN et al., 2009a), (CHEN et al., 2009b), (CHEN et al., 2007), (MENG; BARBOSA, 2006), (HENNICKER; JANISCH; KNAPP, 2010) and (BAUER et al., 2010). Specially, (RAMOS, 2011) focus on the development of deadlock free component-based systems by construction. Nevertheless, it does not develop a refinement or a inheritance relation for components, which is the main purpose of this work. As the authors are aware, this is the first time component inheritance is addressed, and its effect on substitutability is analysed. Some, however, have addressed the concept of component refinement, but based their results on the traces or state base models, as rCOS (Refinement of Component and Object System) (LIU; MORISSET; STOLZ, 2009), (JIFENG; LI; LIU, 2005), (WANG; WANG; ZHAN, 2009), (JIFENG; LI; LIU, 2006), (CHEN et al., 2009a), (CHEN et al., 2009b), and (CHEN et al., 2007). These approaches for component refinement have a very restrictive impact on substitutability since they are either solely based on traces or in pre/post condition analyses.

In rCOS (LIU; MORISSET; STOLZ, 2009) a component has a provided and a required interface and a code associated to each of their method signatures. An interface is a syntactic notion that encompasses typed variable declarations, called fields, and method signatures with input and output parameters alongside with their types. A contract is a black box behaviour model composed by interfaces: it defines for each of them an initial condition that specifies the allowable initial states, a reactive behaviour specification for each method interface, and a protocol, a finite set of allowed traces over the method names. A component is an instance of a contract whose main action can call/use the methods signalised by its interfaces. This approach is distinguished from $\mathcal{B R J C}$ by not treating inputs as an environment decision and outputs as an internal decision, by not analysing behavioural properties in its composition rules and by stating the results on traces instead of the failures model, which compromises substitutability.

The works about rCOS agree that a contract refines another when there is a correspondent refinement between their required and provided interfaces (given in terms of the UTP semantics) and between their traces (traces semantics). The works (LIU; MORISSET; STOLZ, 2009),(CHEN et al., 2009a), (CHEN et al., 2009b) and (CHEN et al., 2007) require that both components have the same provided and required interface, as we assume in our refinement relation, although (JIFENG; LI; LIU, 2005) and (WANG; WANG; ZHAN, 2009) allow interface extension.

In (MENG; BARBOSA, 2006) the CB-MDD foundations are given using the coalgebras theory. Refinement is defined as a transformation of an abstract into a more concrete design. 
A component is a behaviour refinement of another if there exists a forward morphism between them. There, functionality extension appears as an alternative for the lack of inheritance relations, a clearly distinction from our work, as we make a distinction between the two concepts but link them through an evolutionary development approach.

Other works (HENNICKER; JANISCH; KNAPP, 2010) (BAUER et al., 2010) have established their roots in the transition systems theory.(HENNICKER; JANISCH; KNAPP, 2010) defines an I/O labelling as a 3-tuple of outputs, inputs and internal labels used by an I/O-transition system, which encompasses a set of states and transitions. A component is formed of a set of ports and an observable behaviour given in terms of an I/O-transition system. A component refines another if both have the same ports and there is a correspondence between the states of their I/O-transition systems. There is no possibility of functionality extension, since both components must have the same ports and any observable behaviour of one must be possible by the other. (BAUER et al., 2010) uses similar I/O transition systems to define component behaviour. It allows functionality extension by expressing component refinement in terms of a mapping function between states and transitions of two I/O transition systems. It has a similar approach to (MENG; BARBOSA, 2006), as ours stands with that of (HENNICKER; JANISCH; KNAPP, 2010), since our understanding is towards distinguishing refinement from inheritance, putting refinement as a way to achieve non-determinism reduction and inheritance as a way to embed on new system functionalities.

Some works have proposed inheritance relations for behavioural specifications (LISKOV; WING, 1994; WEHRHEIM, 2003; AMERICA, 1991; BOWMAN; DERRICK, 1999; PUNTIGAM, 1996; DIHEGO; ANTONINO; SAMPAIO, 2013). In (AMERICA, 1991; LISKOV; WING, 1994) inheritance relations are defined in terms of invariants over state components and by pre/post conditions over defined methods. The remaining works define subtype relations based on models like failures and failures-divergences (denotational models of CSP), relating refinement with inheritance (WEHRHEIM, 2003). Neither of them differentiates inputs from outputs or considers structural elements besides behavioural specifications. Although (WEHRHEIM, 2003) analyses substitutability, she does relate it with behavioural properties, as deadlock freedom.

As the mentioned works on behavioural inheritance, we also state our relations in the failures model, but we distinguish inputs from outputs, not only by putting them in different sets, but restring the way they can be communicated. (BERTRAND et al., 2011) presents a relation named I/O abstraction that has some sort of connection with convergence. It allows an implementation to input more but restrict it to output less than its abstractions, but it does not consider what happens after the implementation communicates a new input, which clearly weakens substitutability and thus the behavioural properties preservation, as deadlock freedom, in the composition rules. In the same way the relation called ioco (TRETMANS, 1996) allows new inputs to be communicated by an implementation, but as the I/O abstraction relation, it differs from our work by not considering what the implementation does after engaging in a new input. We also highlight an important design decision we have taken in this work: we allow 
functionality extension to be implemented not only in terms of new events but also by those already existing, therefore we allow new-in-context (not only new-in-alphabet) events to be communicated by an inherited component. It gives more flexibility, but presents a challenge to the inheritance verification as we have demonstrated in the construction of an automated strategy for verifying convergence using the FDR3 model checker.

The treatment of inputs as an environment decision and outputs as being internally resolved by the component itself (I/O process definition) is also the approach adopted by (CAVALCANTI; HIERONS, 2013), but differs from us by developing a new semantic model named IOFailures, which is not completely compositional.

From what we have seen and as far as we are aware this is the first time component inheritance relations are developed for a formal and sound CB-MDD approach, with a formal semantics and a refinement relation.

In (ANDREWS, 2001), the authors define an aspect-weaver algorithm for a process algebra based on CSP with process equivalence given in the traces model. Aspects are processes and the weaver works by resolving synchronism; in our strategy, we consider components and aspects in different categories given their distinct nature. We define equivalence in the failures model, which considers refusals in addition to traces. Differently from (ANDREWS, 2001) our approach is compositional, so global properties can be checked locally.

The Protocol Modelling framework (MCNEILE; ROUBTSOVA, 2008) also uses a CSP-like parallelism to weave aspects and supports local reasoning. Nevertheless, it requires specifications to be deterministic, a limitation we have relaxed. It addresses, as in (ANDREWS, 2001), only behavioural constructions. We differentiate by considering both structure and behaviour at a component level, where extensibility and conformance are properly addressed.

This work benefits from the formalisation, given by a denotational semantics, of the aspects theory for a programming language that embodies the key features of join points, pointcuts, and advices (WAND; KICZALES; DUTCHYN, 2004).

\section{Future work}

Although we have obtained important results on component semantics, refinement and specially inheritance, some opportunities remain for future work.

Industrial case studies. Our case studies have shown the use, purpose and motivation for our relations (component refinement and inheritance), but the practical applicability of our approach can only be assessed by the analyses of industrial case studies, which have significant amount of data and complex business logic, such as traffic aviation control and nuclear power plant systems.

Tool support. An essential condition for the $\mathcal{B R J C}$ adoption is the development of a tool to support the specification, refinement and inheritance of components. A further development can enable the suggestion of model evolutions by identifying proper refinement and inheritance 
opportunities.

We also plan to mechanise the proofs of our lemmas and theorems in CSP-Prover (ISOBE; ROGGENBACH, 2008), an interactive theorem prover for CSP based on Isabelle/HOL (NIPKOW; WENZEL; PAULSON, 2002). We expect to develop a tool support for assisted development of component-based specifications with aspects and inheritance support.

Automated serialisation. We have defined a serialised format for I/O processes. At this time, it is generated by hand. Although we can check the correction of this process in FDR, this is an error prone method and therefore demands the development of a tool to mechanise this process for larger specifications.

Broader use of convergence. Behavioural convergence is an independent concept that can be applied beyond CB-MDD. The study of these possibilities constitute a great opportunity for further developments on OhCircus (CAVALCANTI; SAMPAIO; WOODCOCK, 2005) and Event-B (ABRIAL, 2010), among others. 


\section{References}

ABRIAL, J.-R. Modeling in Event-B: system and software engineering. 1st.ed. New York, NY, USA: Cambridge University Press, 2010.

ABRIAL, J.-R. et al. The B-method (software development). In: VDM 91. FORMAL SOFTWARE DEVELOPMENT METHODS. 4TH INTERNATIONAL SYMPOSIUM OF VDM EUROPE PROCEEDINGS. Anais... Springer-Verlag: Berlin: Germany, 1991. v.2, p.398-405.

ALLEN, R.; DOUENCE, R.; GARLAN, D. Specifying and analyzing dynamic software architectures. In: ASTESIANO, E. (Ed.). Fundamental Approaches to Software

Engineering. [S.1.]: Springer Berlin Heidelberg, 1998. p.21-37. (Lecture Notes in Computer Science, v.1382).

ALLEN, R.; GARLAN, D. Formalizing Architectural Connection. In: INTERNATIONAL CONFERENCE ON SOFTWARE ENGINEERING, 16., Los Alamitos, CA, USA.

Proceedings... IEEE Computer Society Press, 1994. p.71-80. (ICSE'94).

ALLEN, R. J. A Formal Approach to Software Architecture. 1997. Tese (Doutorado em Ciência da Computação) — , Pittsburgh, PA, USA. AAI9813815.

AMERICA, P. Designing an Object-Oriented Programming Language with Behavioural Subtyping. In: REX SCHOOL/WORKSHOP ON FOUNDATIONS OF OBJECT-ORIENTED LANGUAGES, London, UK. Proceedings... Springer-Verlag, 1991. p.60-90.

ANDREWS, J. Process-Algebraic Foundations of Aspect-Oriented Programming. In: YONEZAWA, A.; MATSUOKA, S. (Ed.). Metalevel Architectures and Separation of Crosscutting Concerns. [S.1.]: Springer Berlin Heidelberg, 2001. p.187-209. (Lecture Notes in Computer Science, v.2192).

ARBAB, F. Manifold version 2: language reference manual. [S.1.]: Centrum voor Wiskunde en Informatica, Amsterdam, The Netherlands, 1996.

ARBAB, F. Reo: a channel-based coordination model for component composition.

Mathematical. Structures in Comp. Sci., New York, NY, USA, v.14, n.3, p.329-366, June 2004.

ARBAB, F.; RUTTEN, J. A Coinductive Calculus of Component Connectors. In: WIRSING, M.; PATTINSON, D.; HENNICKER, R. (Ed.). Recent Trends in Algebraic Development Techniques. [S.1.]: Springer Berlin Heidelberg, 2003. p.34-55. (Lecture Notes in Computer Science, v.2755).

BAUER, S. et al. On Weak Modal Compatibility, Refinement, and the MIO Workbench. In: ESPARZA, J.; MAJUMDAR, R. (Ed.). Tools and Algorithms for the Construction and Analysis of Systems. [S.1.]: Springer Berlin Heidelberg, 2010. p.175-189. (Lecture Notes in Computer Science, v.6015).

BERTRAND, N. et al. Off-line test selection with test purposes for non-deterministic timed automata. In: TOOLS AND ALGORITHMS FOR THE CONSTRUCTION AND ANALYSIS OF SYSTEMS: PART OF THE JOINT EUROPEAN CONFERENCES ON THEORY AND 
PRACTICE OF SOFTWARE, 17., Berlin, Heidelberg. Proceedings... Springer-Verlag, 2011. p.96-111. (TACAS'11/ETAPS'11).

BIJL, M. van der; RENSINK, A.; TRETMANS, J. Compositional Testing with ioco. In: PETRENKO, A.; ULRICH, A. (Ed.). Formal Approaches to Software Testing. [S.1.]: Springer Berlin Heidelberg, 2004. p.86-100. (Lecture Notes in Computer Science, v.2931).

BOITEN, E. Introducing extra operations in refinement. Formal Aspects of Computing, [S.1.], v.26, n.2, p.305-317, 2014.

BONSANGUE, M. M. et al. A Transition System Semantics for the Control-driven Coordination Language MANIFOLD. Theor. Comput. Sci., Essex, UK, v.240, n.1, p.3-47, June 2000.

BOWMAN, H.; DERRICK, J. A Junction between State Based and Behavioural Specification (Invited Talk). In: SPRINGER-VERLAG, Deventer, The Netherlands. Anais... Kluwer: B.V., 1999. p.213-239.

CAVALCANTI, A.; HIERONS, R. M. Testing with inputs and outputs in CSP. In:

FUNDAMENTAL APPROACHES TO SOFTWARE ENGINEERING, 16., Berlin, Heidelberg. Proceedings... Springer-Verlag, 2013. p.359-374. (FASE'13).

CAVAlCANTI, A. L. C.; SAMPAIO, A. C. A.; WOODCOCK, J. C. P. Unifying Classes and Processes. Software and System Modelling, [S.1.], v.4, n.3, p.277 - 296, 2005.

CHEN, X. et al. A Model of Component-based Programming. In: INTERNATIONAL CONFERENCE ON FUNDAMENTALS OF SOFTWARE ENGINEERING, 2007., Berlin, Heidelberg. Proceedings... Springer-Verlag, 2007. p.191-206. (FSEN'07).

CHEN, X.; HE, J.; LIU, Z. Component Coordination in rCOS. Macau: United Nations University, International Institute for Software Technology, 2006. (335).

CHEN, Z. et al. Refinement and Verification in Component-based Model-driven Design. Sci. Comput. Program., Amsterdam, The Netherlands, The Netherlands, v.74, n.4, p.168-196, Feb. 2009.

CHEN, Z. et al. Refinement and verification in component-based model-driven design. Science of Computer Programming, [S.1.], v.74, 02/2009 2009.

CHOMSKY, N. Aspects of the Theory of Syntax. Cambridge: The MIT Press, 1965.

CLIP2. The Annotated Gnutella Protocol Specification v0.4, Revision 1.6. [S.1.]: The Gnutella Developer Forum (GDF), 2001.

COADY, Y. et al. Using aspectC to Improve the Modularity of Path-specific Customization in Operating System Code. SIGSOFT Softw. Eng. Notes, New York, NY, USA, v.26, n.5, p.88-98, Sept. 2001.

DIHEGO, J. Inheritance and refinement of trustworthy component-based systems. In: AICHERNIG, B. K.; ROSSINI, A. (Ed.). Doctoral Symposium of Formal Methods 2015. [S.1.]: University of Oslo - Department of Informatics, 2015. p.27-31.

DIHEGO, J.; ANTONINO, P.; SAMPAIO, A. Algebraic Laws for Process Subtyping. In: GROVES, L.; SUN, J. (Ed.). Formal Methods and Software Engineering. [S.1.]: Springer Berlin Heidelberg, 2013. p.4-19. (Lecture Notes in Computer Science, v.8144). 
DIHEGO, J.; SAMPAIO, A. Aspect-Oriented Development of Trustworthy Component-based Systems. In: LEUCKER MARTIN, R. C. V. F. D. (Ed.). Theoretical Aspects of Computing ICTAC 2015. [S.1.]: Springer International Publishing, 2015. p.1-20. (Theoretical Computer Science and General Issues, v.9399).

DIHEGO, J.; SAMPAIO, A.; OLIVEIRA, M. Constructive Extensibility of Trustworthy Component-based Systems. In: ANNUAL ACM SYMPOSIUM ON APPLIED COMPUTING, 30., New York, NY, USA. Proceedings... ACM, 2015. p.1808-1814. (SAC '15).

ENDERTON, H. B. Elements of set theory. New York: Academic Press, 1977.

FIADEIRO, J.; LOPES, A. Semantics of architectural connectors. In: BIDOIT, M.; DAUCHET, M. (Ed.). TAPSOFT '97: theory and practice of software development. [S.1.]: Springer Berlin Heidelberg, 1997. p.503-519. (Lecture Notes in Computer Science, v.1214).

FILMAN, R. et al. Aspect-oriented Software Development. 1.ed. [S.1.]: Addison-Wesley Professional, 2004.

FISCHER, C. CSP-OZ: a combination of object-z and csp. In: IFIP TC6 WG6.1

INTERNATIONAL WORKSHOP ON FORMAL METHODS FOR OPEN OBJECT-BASED DISTRIBUTED SYSTEMS, London, UK, UK. Proceedings... Chapman \& Hall: Ltd., 1997. p.423-438.

Formal-Systems. ProBE User Manual. [S.1.]: Formal Systems (Europe) Ltd., 2003.

FORMAL-SYSTEMS. Failures-Divergence Refinement: fdr2 user manual. [S.1.]: Formal Systems (Europe) and Oxford University Computing Laboratory, 2010.

GARLAN, D.; ALLEN, R.; OCKERBLOOM, J. Exploiting Style in Architectural Design Environments. In: ND ACM SIGSOFT SYMPOSIUM ON FOUNDATIONS OF SOFTWARE ENGINEERING, 2., New York, NY, USA. Proceedings... ACM, 1994. p.175-188. (SIGSOFT '94).

GARLAN, D.; MONROE, R.; WILE, D. Acme: an architecture description interchange language. In: CONFERENCE OF THE CENTRE FOR ADVANCED STUDIES ON COLLABORATIVE RESEARCH, 1997. Proceedings... IBM Press, 1997. p.7-. (CASCON '97).

GEPPERT, A.; TOMBROS, D.; DITTRICH, K. R. Defining the Semantics of Reactive Components in Event-driven Workflow Execution with Event Histories. Inf. Syst., Oxford, UK, UK, v.23, n.3-4, p.235-252, May 1998.

GIBSON-ROBINSON, T. et al. FDR3 - A Modern Refinement Checker for CSP. In: TOOLS AND ALGORITHMS FOR THE CONSTRUCTION AND ANALYSIS OF SYSTEMS - 20TH INTERNATIONAL CONFERENCE, TACAS 2014, 2014, GRENOBLE, FRANCE, APRIL 5-13, 2014. PROCEEDINGS. Anais... [S.1.: s.n.], 2014. p.187-201.

GRAY, D. N. et al. Modern Languages and Microsoft's Component Object Model. Commun. ACM, New York, NY, USA, v.41, n.5, p.55-65, May 1998.

HE, J.; LI, X.; LIU, Z. rCOS: a refinement calculus of object systems. Theoretical Computer Science, [S.1.], v.365, n.1-2, p.109-142, 2006. 
HENNICKER, R.; JANISCH, S.; KNAPP, A. Refinement of Components in Connection-safe Assemblies with Synchronous and Asynchronous Communication. In: MONTEREY CONFERENCE ON FOUNDATIONS OF COMPUTER SOFTWARE: FUTURE TRENDS AND TECHNIQUES FOR DEVELOPMENT, 15., Berlin, Heidelberg. Proceedings... Springer-Verlag, 2010. p.154-180. (Monterey’08).

HOARE, C. A. R. In: COMMUNICATING SEQUENTIAL PROCESSES, New York, NY, USA. Anais... ACM, 1978. v.21, p.666-677.

ISOBE, Y.; ROGGENBACH, M. CSP-Prover: a proof tool for the verification of scalable concurrent systems. Computer Software, [S.1.], v.25, n.4, 2008.

JIFENG, H.; LI, X.; LIU, Z. Component-based Software Engineering. In: SECOND INTERNATIONAL CONFERENCE ON THEORETICAL ASPECTS OF COMPUTING, Berlin, Heidelberg. Proceedings... Springer-Verlag, 2005. p.70-95. (ICTAC'05).

JIFENG, H.; LI, X.; LIU, Z. rCOS: a refinement calculus of object systems. Theoretical Computer Science, [S.1.], v.365, p.109 - 142, 2006. Formal Methods for Components and Objects Formal Methods for Components and Objects.

JONES, C. B. Systematic Software Development Using VDM (2Nd Ed.). Upper Saddle River, NJ, USA: Prentice-Hall, Inc., 1990.

KICZALES, G. et al. Aspect-oriented programming. In: AKşIT, M.; MATSUOKA, S. (Ed.). ECOOP'97 - Object-Oriented Programming. [S.1.]: Springer Berlin Heidelberg, 1997. p.220-242. (Lecture Notes in Computer Science, v.1241).

KICZALES, G. et al. An Overview of AspectJ. In: EUROPEAN CONFERENCE ON OBJECT-ORIENTED PROGRAMMING, 15., London, UK, UK. Proceedings... Springer-Verlag, 2001. p.327-353. (ECOOP'01).

KLINGBERG, T.; MANFREDI, R. Gnutella 0.6. [S.1.]: The Gnutella Developer Forum (GDF), June 2002.

LAMPORT, L. The Temporal Logic of Actions. ACM Trans. Program. Lang. Syst., New York, NY, USA, v.16, n.3, p.872-923, May 1994.

LANO, K. The B Language and Method: a guide to practical formal development. 1st.ed. Secaucus, NJ, USA: Springer-Verlag New York, Inc., 1996.

LISKOV, B. Keynote Address - Data Abstraction and Hierarchy. SIGPLAN Not., New York, NY, USA, v.23, n.5, p.17-34, Jan. 1987.

LISKOV, B. H.; WING, J. M. A behavioral notion of subtyping. ACM Trans. Program. Lang. Syst., New York, NY, USA, v.16, n.6, p.1811-1841, 1994.

LIU, Z.; JIFENG, H.; LI, X. rCOS: refinement of component and object systems. In: BOER, F. de et al. (Ed.). Formal Methods for Components and Objects. [S.l.]: Springer Berlin Heidelberg, 2005. p.183-221. (Lecture Notes in Computer Science, v.3657).

LIU, Z.; MORISSET, C.; STOLZ, V. rCOS: theory and tool for component-based model driven development. In: THIRD IPM INTERNATIONAL CONFERENCE ON FUNDAMENTALS OF SOFTWARE ENGINEERING, Berlin, Heidelberg. Proceedings... Springer-Verlag, 2009. p.62-80. (FSEN'09). 
LUCKHAM, D. C. et al. Specification and Analysis of System Architecture Using Rapide. IEEE Trans. Softw. Eng., Piscataway, NJ, USA, v.21, n.4, p.336-355, Apr. 1995.

MAGEE, J. et al. Specifying Distributed Software Architectures. In: EUROPEAN SOFTWARE ENGINEERING CONFERENCE, 5., London, UK, UK. Proceedings... Springer-Verlag, 1995. p.137-153.

MCNEILE, A.; ROUBTSOVA, E. CSP Parallel Composition of Aspect Models. In: AOSD WORKSHOP ON ASPECT-ORIENTED MODELING, 2008., New York, NY, USA.

Proceedings... ACM, 2008. p.13-18. (AOM '08).

MEDVIDOVIC, N.; ROSENBLUM, D. S. Assessing the Suitability of a Standard Design Method for Modeling Software Architectures. In: TC2 FIRST WORKING IFIP CONFERENCE ON SOFTWARE ARCHITECTURE (WICSA1), Deventer, The Netherlands, The Netherlands. Proceedings... Kluwer: B.V., 1999. p.161-182. (WICSA1).

MEDVIDOVIC, N.; TAYLOR, R. N. A Classification and Comparison Framework for Software Architecture Description Languages. IEEE Trans. Softw. Eng., Piscataway, NJ, USA, v.26, n.1, p.70-93, Jan. 2000.

MENG, S.; BARBOSA, L. S. Components As Coalgebras: the refinement dimension. Theor. Comput. Sci., Essex, UK, v.351, n.2, p.276-294, Feb. 2006.

MILNER, R. A Calculus of Communicating Systems. Secaucus, NJ, USA: Springer-Verlag New York, Inc., 1982.

MILNER, R. Communication and Concurrency. [S.1.]: Prentice-Hall, 1989.

NIPKOW, T.; WENZEL, M.; PAULSON, L. C. Isabelle/HOL: a proof assistant for higher-order logic. Berlin, Heidelberg: Springer-Verlag, 2002.

Oliveira, M. and Sampaio, A. and Antonino,P. and Dihego, J. and Filho, M. C. and Bryans, J. Compositional Analysis and Design of CML Models. [S.1.]: Comprehensive Modelling for Advanced Systems of Systems, 2014.

PUNTIGAM, F. Types for Active Objects Based on Trace Semantics. In: FMOODS 96. Proceedings... Chapman and Hall, 1996. p.4-19.

RAMOS, R.; SAMPAIO, A.; MOTA, A. Systematic Development of Trustworthy Component Systems. In: WORLD CONGRESS ON FORMAL METHODS, 2. Anais... Springer, 2009. p.140-156. (Lecture Notes in Computer Science, v.5850).

RAMOS, R. T. Systematic Development of Trustworthy Component-based Systems. 2011. Tese (Doutorado em Ciência da Computação) - Center of Informatics - Federal University of Pernambuco, Brazil.

ROGERSON, D. Inside COM. Redmond, WA, USA: Microsoft Press, 1997.

ROSCOE, A. W. Theory and Practice of Concurrency. [S.1.]: Prentice-Hall, 1998. (Prentice-Hall Series in Computer Science).

ROSCOE, A. W. The pursuit of buffer tolerance. [S.1.]: Oxford University, 2005. 
ROSCOE, B.; DATHI, N. The pursuit of deadlock freedom. Information and Computation, [S.1.], v.75, n.3, p.289-327, Dec. 1987.

RUBINGER, A. L.; BURKE, B. Enterprise JavaBeans 3.1. [S.1.]: O’Reilly Media, Inc., 2010.

RUMBAUGH, J.; JACOBSON, I.; BOOCH, G. Unified Modeling Language Reference

Manual, The (2Nd Edition). [S.1.]: Pearson Higher Education, 2004.

SCHOLLMEIER, R. [16] A Definition of Peer-to-Peer Networking for the Classification of Peer-to-Peer Architectures and Applications. In: FIRST INTERNATIONAL CONFERENCE ON PEER-TO-PEER COMPUTING, Washington, DC, USA. Proceedings... IEEE Computer Society, 2001. p.101-. (P2P '01).

SELIC, B. Using UML for modeling complex real-time systems. In: MUELLER, F.; BESTAVROS, A. (Ed.). Languages, Compilers, and Tools for Embedded Systems. [S.1.]: Springer Berlin Heidelberg, 1998. p.250-260. (Lecture Notes in Computer Science, v.1474).

SELIC, B.; RUMBAUGH, J. Using UML for Modeling Complex RealTime Systems. [S.1.]: Rational Software Corporation, 1998.

SPIVEY, J. M. The $\mathbf{Z}$ notation: a reference manual. Upper Saddle River, NJ, USA: Prentice-Hall, Inc., 1989.

STEINMETZ, R.; WEHRLE, K. 2. What Is This "Peer-to-Peer" About? In: STEINMETZ, R.; WEHRLE, K. (Ed.). Peer-to-Peer Systems and Applications. [S.1.]: Springer Berlin Heidelberg, 2005. p.9-16. (Lecture Notes in Computer Science, v.3485).

SUN, J.; LIU, Y.; DONG, J. Model Checking CSP Revisited: introducing a process analysis toolkit. In: MARGARIA, T.; STEFFEN, B. (Ed.). Leveraging Applications of Formal Methods, Verification and Validation. [S.1.]: Springer Berlin Heidelberg, 2008. p.307-322. (Communications in Computer and Information Science, v.17).

SZYPERSKI, C. Component Software: beyond object-oriented programming. New York, NY, USA: ACM Press/Addison-Wesley Publishing Co., 1998.

TRETMANS, J. Test Generation with Inputs, Outputs and Repetitive Quiescence. Software Concepts and Tools, [S.1.], v.17, n.3, p.103-120, 1996.

WAND, M.; KICZALES, G.; DUTCHYN, C. A Semantics for Advice and Dynamic Join Points in Aspect-oriented Programming. ACM Trans. Program. Lang. Syst., New York, NY, USA, v.26, n.5, p.890-910, Sept. 2004.

WANG, Z.; WANG, H.; ZHAN, N. Towards a theory of refinement of component-based systems. [S.1.]: UNU-IIST, 2009. Report. (427).

WEGNER, P.; ZDONIK, S. B. Inheritance as an Incremental Modification Mechanism or What Like Is and Isn't Like. In: EUROPEAN CONFERENCE ON OBJECT-ORIENTED PROGRAMMING, London, UK. Proceedings... Springer-Verlag, 1988. p.55-77. (ECOOP '88).

WEHRHEIM, H. Checking Behavioural Subtypes via Refinement. 2002.

WEHRHEIM, H. Behavioral Subtyping Relations for Active Objects. Form. Methods Syst. Des., Hingham, MA, USA, v.23, n.2, p.143-170, 2003. 
WOODCOCK, J.; DAVIES, J. Using Z: specification, refinement, and proof. Upper Saddle River, NJ, USA: Prentice-Hall, Inc., 1996. 
Appendix 


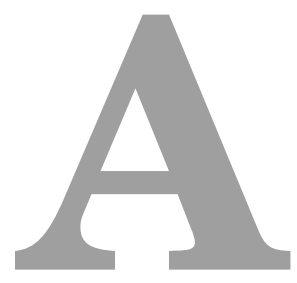

\section{I/O process serialization}

This appendix shows the serialised representation of the I/O processes ATM (Section 5.1) and HC_BOT (Section 6.3).

\section{ATM serialisation}

ATM_serial $=$

$<($ start, <atm_cus.in.card.(1,true),atm_cus.in.card.(2,true),atm_cus.in.card.(1,false),atm_cus.in.card.(2,false) $>, 0)$,

(atm_cus.in.card.(1,true), <atm_cus.in.password.1, atm_cus.in.password.2>, 1),

(atm_cus.in.password.1,<atm_cus.out.error.wrongPin,atm_cus.out.successlogin,atm_cus.out.error.wrongCard>,2),

(atm_cus.out.error.wrongPin, <end $>, 3$ ),

(end, $<>, 4)$,

(atm_cus.out.successlogin, <atm_cus.in.deposit.0, atm_cus.in.deposit.1, atm_cus.in.deposit.2,

atm_cus.in.withdraw.0, atm_cus.in.withdraw.1, atm_cus.in.withdraw.2, atm_cus.in.transfer.1.0,atm_cus.in.transfer.1.1, atm_cus.in.transfer.1.2, atm_cus.in.transfer.2.0,atm_cus.in.transfer.2.1, atm_cus.in.transfer.2.2, atm_cus.in.askBalance>,3),

(atm_cus.in.deposit.0, $<$ atm_cus.out.succ_dep.0>,4),

(atm_cus.out.succ_dep.0, <end $>, 5$ ),

(end, $<>, 6$ ),

(atm_cus.in.deposit.1,<atm_cus.out.succ_dep.1>,4),

(atm_cus.out.succ_dep.1, <end $>, 5$ ),

(end, <>,6),

(atm_cus.in.deposit.2,<atm_cus.out.succ_dep.2>,4),

(atm_cus.out.succ_dep.2, <end $>, 5$ ),

(end, $<>, 6$ ),

(atm_cus.in.withdraw.0,<atm_cus.out.succ_wit.0, atm_cus.out.error.unsufBalance>,4),

(atm_cus.out.succ_wit.0, <end $>, 5$ ),

(end, $<>, 6$ ),

(atm_cus.out.error.unsufBalance, $<$ end $>, 5$ ),

(end, $<>, 6$ ),

(atm_cus.in.withdraw.1,<atm_cus.out.succ_wit.1, atm_cus.out.error.unsufBalance>,4),

(atm_cus.out.succ_wit.1, <end >, 5),

(end, $<>, 6$ ),

(atm_cus.out.error.unsufBalance, $<$ end $>, 5$ ),

(end, $<>, 6$ ),

(atm_cus.in.withdraw.2,<atm_cus.out.succ_wit.2, atm_cus.out.error.unsufBalance>,4),

(atm_cus.out.succ_wit.2, <end>, 5),

(end, <>, 6),

(atm_cus.out.error.unsufBalance, $<$ end $>, 5$ ), 
(end, $<>, 6)$

(atm_cus.in.transfer.1.0,<atm_cus.out.succ_tra.1.0,atm_cus.out.error.unsufBalance, atm_cus.out.error.wrongDestNumber $>$,4), (atm_cus.out.succ_tra.1.0, <end>, 5),

(end, $<>, 6$ ),

(atm_cus.out.error.unsufBalance, $<\mathrm{end}>, 5$ ),

(end, <>,6),

(atm_cus.out.error.wrongDestNumber,<end $>, 5$ ),

(end, $<>, 6)$,

(atm_cus.in.transfer.1.1,<atm_cus.out.succ_tra.1.1, atm_cus.out.error.unsufBalance, atm_cus.out.error.wrongDestNumber $>$,4),

(atm_cus.out.succ_tra.1.1, <end >, 5),

(end, $<>, 6$ ),

(atm_cus.out.error.unsufBalance, $<$ end $>, 5$ ),

(end, $<>, 6$ ),

(atm_cus.out.error.wrongDestNumber,<end $>, 5$ ),

(end, $<>, 6)$,

(atm_cus.in.transfer.1.2,<atm_cus.out.succ_tra.1.2, atm_cus.out.error.unsufBalance, atm_cus.out.error.wrongDestNumber>,4), (atm_cus.out.succ_tra.1.2, <end>, 5),

(end, $<>, 6$ ),

(atm_cus.out.error.unsufBalance, $<\mathrm{end}>, 5$ ),

(end, <>,6),

(atm_cus.out.error.wrongDestNumber,<end $>, 5$ ),

(end, $<>, 6)$,

(atm_cus.in.transfer.2.0,<atm_cus.out.succ_tra.2.0, atm_cus.out.error.unsufBalance, atm_cus.out.error.wrongDestNumber $>$,4),

(atm_cus.out.succ_tra.2.0, <end >, 5),

(end, $<>, 6$ ),

(atm_cus.out.error.unsufBalance, $<$ end $>, 5$ ),

(end, $<>, 6$ ),

(atm_cus.out.error.wrongDestNumber,<end $>, 5$ ),

(end, $<>, 6)$,

(atm_cus.in.transfer.2.1,<atm_cus.out.succ_tra.2.1, atm_cus.out.error.unsufBalance, atm_cus.out.error.wrongDestNumber>,4), (atm_cus.out.succ_tra.2.1, <end>, 5),

(end, $<>, 6$ ),

(atm_cus.out.error.unsufBalance, $<\mathrm{end}>, 5$ ),

(end, <>,6),

(atm_cus.out.error.wrongDestNumber,<end $>, 5$ ),

(end, $<>, 6)$,

(atm_cus.in.transfer.2.2,<atm_cus.out.succ_tra.2.2, atm_cus.out.error.unsufBalance, atm_cus.out.error.wrongDestNumber $>$,4),

(atm_cus.out.succ_tra.2.2, <end>, 5),

(end, $<>, 6$ ),

(atm_cus.out.error.unsufBalance, $<$ end $>, 5$ ),

(end, <>,6),

(atm_cus.out.error.wrongDestNumber,<end $>, 5$ ),

(end, $<>, 6)$,

(atm_cus.in.askBalance,<atm_cus.out.balance.0, atm_cus.out.balance.1, atm_cus.out.balance.2>,4),

(atm_cus.out.balance.0, <end>, 5),

(end, $<>, 6$ ),

(atm_cus.out.balance. $1,<\mathrm{end}>, 5$ ),

(end, $<>, 6)$,

(atm_cus.out.balance.2,<end $>, 5$ ),

(end, $<>, 6)$,

(atm_cus.out.error.wrongCard,<end $>, 3$ ),

(end, $<>, 4)$,

(atm_cus.in.password.2,<atm_cus.out.error.wrongPin,atm_cus.out.successlogin,atm_cus.out.error.wrongCard $>, 2$ ),

(atm_cus.out.error.wrongPin, <end $>, 3$ ),

(end, $<>, 4)$,

(atm_cus.out.successlogin,<atm_cus.in.deposit.0, atm_cus.in.deposit.1, atm_cus.in.deposit.2,

atm_cus.in.withdraw.0, atm_cus.in.withdraw.1, atm_cus.in.withdraw.2,

atm_cus.in.transfer.1.0,atm_cus.in.transfer.1.1, atm_cus.in.transfer.1.2,

atm_cus.in.transfer.2.0,atm_cus.in.transfer.2.1, atm_cus.in.transfer.2.2, 
atm_cus.in.askBalance $>, 3)$,

(atm_cus.in.deposit.0,<atm_cus.out.succ_dep.0>,4),

(atm_cus.out.succ_dep.0, <end >,5),

(end, $<>, 6$ ),

(atm_cus.in.deposit.1,<atm_cus.out.succ_dep.1>,4),

(atm_cus.out.succ_dep.1, <end >,5),

(end, $<>, 6$ ),

(atm_cus.in.deposit.2,<atm_cus.out.succ_dep.2>,4),

(atm_cus.out.succ_dep.2, <end >,5),

(end, $<>, 6$ ),

(atm_cus.in.withdraw.0,<atm_cus.out.succ_wit.0,atm_cus.out.error.unsufBalance $>, 4$ ),

(atm_cus.out.succ_wit.0, <end >, 5),

(end, <>, 6),

(atm_cus.out.error.unsufBalance, $<$ end $>, 5$ ),

(end, $<>, 6)$,

(atm_cus.in.withdraw.1,<atm_cus.out.succ_wit.1, atm_cus.out.error.unsufBalance $>, 4$ ),

(atm_cus.out.succ_wit.1, <end >, 5),

(end, <>, 6),

(atm_cus.out.error.unsufBalance, $<$ end $>, 5$ ),

(end, $<>, 6$ ),

(atm_cus.in.withdraw.2,<atm_cus.out.succ_wit.2, atm_cus.out.error.unsufBalance>,4),

(atm_cus.out.succ_wit.2, <end>, 5),

(end, <>, 6),

(atm_cus.out.error.unsufBalance, $<$ end $>, 5$ ),

(end, $<>, 6)$,

(atm_cus.in.transfer.1.0,<atm_cus.out.succ_tra.1.0,atm_cus.out.error.unsufBalance, atm_cus.out.error.wrongDestNumber>,4),

(atm_cus.out.succ_tra.1.0, <end $>, 5)$,

(end, <>, 6),

(atm_cus.out.error.unsufBalance, $<$ end $>, 5$ ),

(end, $<>, 6)$,

(atm_cus.out.error.wrongDestNumber, <end $>, 5$ ),

(end, $<>, 6)$,

(atm_cus.in.transfer.1.1,<atm_cus.out.succ_tra.1.1,atm_cus.out.error.unsufBalance, atm_cus.out.error.wrongDestNumber>,4),

(atm_cus.out.succ_tra.1.1, <end >, 5),

(end, <>, 6),

(atm_cus.out.error.unsufBalance, $<$ end $>, 5$ ),

(end, $<>, 6$ ),

(atm_cus.out.error.wrongDestNumber,<end>,5),

(end, $<>, 6$ ),

(atm_cus.in.transfer.1.2,<atm_cus.out.succ_tra.1.2,atm_cus.out.error.unsufBalance, atm_cus.out.error.wrongDestNumber>,4), (atm_cus.out.succ_tra.1.2, <end >, 5),

(end, $<>, 6$ ),

(atm_cus.out.error.unsufBalance, $<$ end $>, 5)$,

(end, $<>, 6)$,

(atm_cus.out.error.wrongDestNumber,<end $>, 5$ ),

(end, $<>, 6)$,

(atm_cus.in.transfer.2.0,<atm_cus.out.succ_tra.2.0,atm_cus.out.error.unsufBalance, atm_cus.out.error.wrongDestNumber $>, 4)$,

(atm_cus.out.succ_tra.2.0, <end >, 5),

(end, $<>, 6)$,

(atm_cus.out.error.unsufBalance, $<$ end $>, 5)$,

(end, $<>, 6)$,

(atm_cus.out.error.wrongDestNumber,<end $>, 5)$,

(end, $<>, 6)$,

(atm_cus.in.transfer.2.1,<atm_cus.out.succ_tra.2.1,atm_cus.out.error.unsufBalance, atm_cus.out.error.wrongDestNumber>,4), (atm_cus.out.succ_tra.2.1, <end >, 5),

(end, <>, 6),

(atm_cus.out.error.unsufBalance, $<$ end $>, 5$ ),

(end, $<>, 6$ ),

(atm_cus.out.error.wrongDestNumber,<end >,5), 
(end, $<>, 6)$,

(atm_cus.in.transfer.2.2,<atm_cus.out.succ_tra.2.2, atm_cus.out.error.unsufBalance, atm_cus.out.error.wrongDestNumber $>$,4), (atm_cus.out.succ_tra.2.2, <end>, 5),

(end, $<>, 6$ ),

(atm_cus.out.error.unsufBalance, $<$ end $>, 5$ ),

(end, $<>, 6$ ),

(atm_cus.out.error.wrongDestNumber,<end $>, 5$ ),

(end, $<>, 6)$,

(atm_cus.in.askBalance,<atm_cus.out.balance.0,atm_cus.out.balance.1, atm_cus.out.balance.2>,4),

(atm_cus.out.balance. $0,<\mathrm{end}>, 5$ ),

(end, $<>, 6$ ),

(atm_cus.out.balance.1,<end>,5),

(end, <>,6),

(atm_cus.out.balance.2,<end>,5),

(end, $<>, 6)$,

(atm_cus.out.error.wrongCard,<end $>, 3$ ),

(end, $<>, 4)$,

(atm_cus.in.card.(2,true), <atm_cus.in.password.1, atm_cus.in.password.2>, 1),

(atm_cus.in.password.1,<atm_cus.out.error.wrongPin,atm_cus.out.successlogin,atm_cus.out.error.wrongCard $>, 2$ ),

(atm_cus.out.error.wrongPin, <end $>, 3$ ),

(end, $<>, 4)$,

(atm_cus.out.successlogin, <atm_cus.in.deposit.0, atm_cus.in.deposit.1, atm_cus.in.deposit.2,

atm_cus.in.withdraw.0, atm_cus.in.withdraw.1, atm_cus.in.withdraw.2,

atm_cus.in.transfer.1.0,atm_cus.in.transfer.1.1, atm_cus.in.transfer.1.2,

atm_cus.in.transfer.2.0,atm_cus.in.transfer.2.1, atm_cus.in.transfer.2.2,

atm_cus.in.askBalance>,3),

(atm_cus.in.deposit.0,<atm_cus.out.succ_dep.0>,4),

(atm_cus.out.succ_dep.0, <end >,5),

(end, $<>, 6$ ),

(atm_cus.in.deposit.1,<atm_cus.out.succ_dep.1>,4),

(atm_cus.out.succ_dep.1, <end $>, 5$ ),

(end, $<>, 6)$,

(atm_cus.in.deposit.2,<atm_cus.out.succ_dep.2>,4),

(atm_cus.out.succ_dep.2, <end $>, 5$ ),

(end, $<>, 6)$,

(atm_cus.in.withdraw.0,<atm_cus.out.succ_wit.0, atm_cus.out.error.unsufBalance>,4),

(atm_cus.out.succ_wit.0, <end $>, 5$ ),

(end, $<>, 6$ ),

(atm_cus.out.error.unsufBalance, $<$ end $>, 5$ ),

(end, <>,6),

(atm_cus.in.withdraw.1,<atm_cus.out.succ_wit.1, atm_cus.out.error.unsufBalance>,4),

(atm_cus.out.succ_wit.1, <end $>, 5$ ),

(end, $<>, 6$ ),

(atm_cus.out.error.unsufBalance, $<$ end $>, 5$ ),

(end, $<>, 6$ ),

(atm_cus.in.withdraw.2,<atm_cus.out.succ_wit.2, atm_cus.out.error.unsufBalance >,4),

(atm_cus.out.succ_wit.2, <end $>, 5$ ),

(end, $<>, 6$ ),

(atm_cus.out.error.unsufBalance, $<$ end $>, 5$ ),

(end, $<>, 6)$,

(atm_cus.in.transfer.1.0,<atm_cus.out.succ_tra.1.0,atm_cus.out.error.unsufBalance, atm_cus.out.error.wrongDestNumber>,4), (atm_cus.out.succ_tra.1.0, <end $>, 5$ ),

(end, $<>, 6$ ),

(atm_cus.out.error.unsufBalance, $<$ end $>, 5)$,

(end, <>,6),

(atm_cus.out.error.wrongDestNumber, $<$ end $>, 5$ ),

(end, $<>, 6)$,

(atm_cus.in.transfer.1.1,<atm_cus.out.succ_tra.1.1, atm_cus.out.error.unsufBalance, atm_cus.out.error.wrongDestNumber>,4), 
(atm_cus.out.succ_tra.1.1, <end >, 5),

(end, $<>, 6$ ),

(atm_cus.out.error.unsufBalance, $<$ end $>, 5$ ),

(end, $<>, 6$ ),

(atm_cus.out.error.wrongDestNumber,<end $>, 5$ ),

(end, $<>, 6$ ),

(atm_cus.in.transfer.1.2,<atm_cus.out.succ_tra.1.2,atm_cus.out.error.unsufBalance, atm_cus.out.error.wrongDestNumber>,4), (atm_cus.out.succ_tra.1.2, <end $>, 5)$,

(end, <>, 6),

(atm_cus.out.error.unsufBalance, $<$ end $>, 5)$,

(end, $<>, 6)$,

(atm_cus.out.error.wrongDestNumber,<end >,5),

(end, $<>, 6$ ),

(atm_cus.in.transfer.2.0,<atm_cus.out.succ_tra.2.0,atm_cus.out.error.unsufBalance, atm_cus.out.error.wrongDestNumber $>$,4),

(atm_cus.out.succ_tra.2.0, <end $>, 5$ ),

(end, $<>, 6$ ),

( atm_cus.out.error.unsufBalance, $<$ end $>, 5$ ),

(end, $<>, 6$ ),

(atm_cus.out.error.wrongDestNumber,<end $>, 5$ ),

(end, $<>, 6$ ),

(atm_cus.in.transfer.2.1,<atm_cus.out.succ_tra.2.1, atm_cus.out.error.unsufBalance, atm_cus.out.error.wrongDestNumber>,4), (atm_cus.out.succ_tra.2.1, <end $>, 5)$,

(end, <>, 6),

(atm_cus.out.error.unsufBalance, $<$ end $>, 5)$,

(end, $<>, 6)$,

(atm_cus.out.error.wrongDestNumber,<end $>, 5$ ),

(end, $<>, 6)$,

(atm_cus.in.transfer.2.2,<atm_cus.out.succ_tra.2.2, atm_cus.out.error.unsufBalance, atm_cus.out.error.wrongDestNumber>,4),

(atm_cus.out.succ_tra.2.2, <end $>, 5$ ),

(end, $<>, 6)$,

(atm_cus.out.error.unsufBalance,<end $>, 5$ ),

(end, $<>, 6$ ),

(atm_cus.out.error.wrongDestNumber,<end $>, 5$ ),

(end, $<>, 6)$,

(atm_cus.in.askBalance, $<$ atm_cus.out.balance.0,atm_cus.out.balance.1, atm_cus.out.balance.2>,4),

(atm_cus.out.balance.0, <end $>, 5$ ),

(end, $<>, 6$ ),

(atm_cus.out.balance.1,<end $>, 5$ ),

(end, $<>, 6$ ),

(atm_cus.out.balance.2,<end $>, 5$ ),

(end, $<>, 6$ ),

(atm_cus.out.error.wrongCard, <end >,3),

(end, $<>, 4$ ),

(atm_cus.in.password.2,<atm_cus.out.error.wrongPin,atm_cus.out.successlogin,atm_cus.out.error.wrongCard $>, 2$ ),

(atm_cus.out.error.wrongPin, <end $>, 3$ ),

(end, $<>, 4)$,

(atm_cus.out.successlogin,<atm_cus.in.deposit.0,atm_cus.in.deposit.1, atm_cus.in.deposit.2,

atm_cus.in.withdraw.0, atm_cus.in.withdraw.1, atm_cus.in.withdraw.2,

atm_cus.in.transfer.1.0,atm_cus.in.transfer.1.1, atm_cus.in.transfer.1.2,

atm_cus.in.transfer.2.0,atm_cus.in.transfer.2.1, atm_cus.in.transfer.2.2,

atm_cus.in.askBalance>,3),

(atm_cus.in.deposit.0,<atm_cus.out.succ_dep.0>,4),

(atm_cus.out.succ_dep.0, <end >,5),

(end, $<>, 6$ ),

(atm_cus.in.deposit.1,<atm_cus.out.succ_dep.1>,4),

(atm_cus.out.succ_dep.1, <end>,5),

(end, $<>, 6$ ),

(atm_cus.in.deposit.2,<atm_cus.out.succ_dep.2>,4),

(atm_cus.out.succ_dep.2, <end>,5), 
(end, $<>, 6)$

(atm_cus.in.withdraw.0,<atm_cus.out.succ_wit.0, atm_cus.out.error.unsufBalance >,4),

(atm_cus.out.succ_wit.0, <end $>, 5$ ),

(end, $<>, 6$ ),

(atm_cus.out.error.unsufBalance, $<$ end $>, 5$ ),

(end, <>,6),

(atm_cus.in.withdraw.1,<atm_cus.out.succ_wit.1, atm_cus.out.error.unsufBalance>,4),

(atm_cus.out.succ_wit.1, <end >, 5),

(end, $<>, 6$ ),

(atm_cus.out.error.unsufBalance, $<$ end $>, 5$ ),

(end, $<>, 6$ ),

(atm_cus.in.withdraw.2,<atm_cus.out.succ_wit.2, atm_cus.out.error.unsufBalance>,4),

(atm_cus.out.succ_wit.2, <end $>, 5$ ),

(end, $<>, 6$ ),

(atm_cus.out.error.unsufBalance, $<$ end $>, 5$ ),

(end, $<>, 6)$,

(atm_cus.in.transfer.1.0,<atm_cus.out.succ_tra.1.0, atm_cus.out.error.unsufBalance, atm_cus.out.error.wrongDestNumber $>$,4),

(atm_cus.out.succ_tra.1.0, <end >, 5),

(end, $<>, 6)$,

( atm_cus.out.error.unsufBalance, $<$ end $>, 5$ ),

(end,<>,6),

(atm_cus.out.error.wrongDestNumber,<end $>, 5$ ),

(end, $<>, 6$ ),

(atm_cus.in.transfer.1.1,<atm_cus.out.succ_tra.1.1, atm_cus.out.error.unsufBalance, atm_cus.out.error.wrongDestNumber $>$,4), (atm_cus.out.succ_tra.1.1, <end $>, 5$ ),

(end, $<>, 6$ ),

( atm_cus.out.error.unsufBalance,<end $>, 5$ ),

(end, <>,6),

(atm_cus.out.error.wrongDestNumber,<end $>, 5$ ),

(end, $<>, 6$ ),

(atm_cus.in.transfer.1.2,<atm_cus.out.succ_tra.1.2, atm_cus.out.error.unsufBalance, atm_cus.out.error.wrongDestNumber $>$,4), (atm_cus.out.succ_tra.1.2, <end>, 5),

(end, $<>, 6$ ),

( atm_cus.out.error.unsufBalance, $<$ end $>, 5$ ),

(end, <>,6),

(atm_cus.out.error.wrongDestNumber, <end $>, 5$ ),

(end, $<>, 6$ ),

(atm_cus.in.transfer.2.0,<atm_cus.out.succ_tra.2.0,atm_cus.out.error.unsufBalance, atm_cus.out.error.wrongDestNumber $>$,4), (atm_cus.out.succ_tra.2.0, <end $>, 5$ ),

(end, $<>, 6$ ),

( atm_cus.out.error.unsufBalance,<end $>, 5$ ),

(end, $<>, 6$ ),

(atm_cus.out.error.wrongDestNumber,<end $>, 5$ ),

(end, $<>, 6$ ),

(atm_cus.in.transfer.2.1,<atm_cus.out.succ_tra.2.1, atm_cus.out.error.unsufBalance, atm_cus.out.error.wrongDestNumber $>$,4), (atm_cus.out.succ_tra.2.1, <end>, 5),

(end, $<>, 6$ ),

( atm_cus.out.error.unsufBalance, $<$ end $>, 5$ ),

(end, $<>, 6$ ),

(atm_cus.out.error.wrongDestNumber,<end $>, 5$ ),

(end, $<>, 6$ ),

(atm_cus.in.transfer.2.2,<atm_cus.out.succ_tra.2.2, atm_cus.out.error.unsufBalance, atm_cus.out.error.wrongDestNumber $>$,4), (atm_cus.out.succ_tra.2.2, <end>, 5),

(end, $<>, 6$ ),

( atm_cus.out.error.unsufBalance, $<\mathrm{end}>, 5$ ),

(end, $<>, 6$ ),

(atm_cus.out.error.wrongDestNumber,<end $>, 5$ ),

(end, $<>, 6$ ),

(atm_cus.in.askBalance,<atm_cus.out.balance.0, atm_cus.out.balance.1, atm_cus.out.balance.2>,4), 


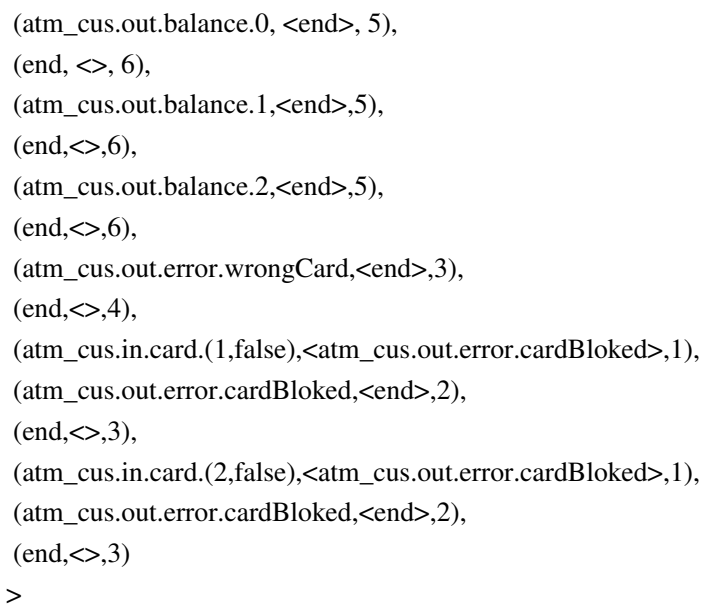

\section{HC BOT serialisation}

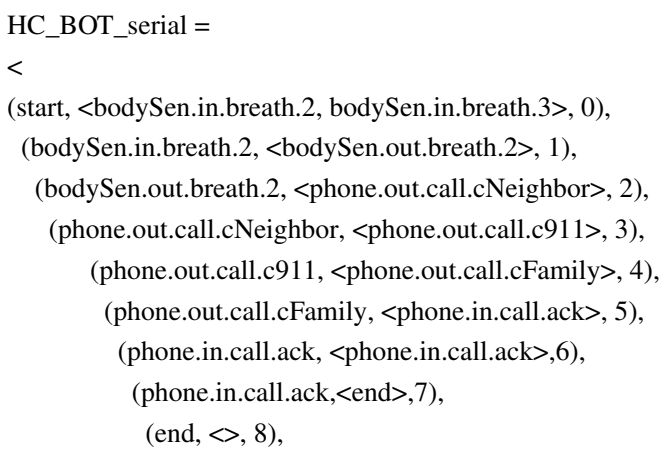

(bodySen.in.breath.3, <voiceRec.in.cough.true, voiceRec.in.cough.false>, 1),

(voiceRec.in.cough.true,<bodySen.in.bodyTemp.38,bodySen.in.bodyTemp.39>,2),

(bodySen.in.bodyTemp.38,<bodySen.in.bloodGlucose.low, bodySen.in.bloodGlucose.nor, bodySen.in.bloodGlucose.threshold, bodySen.in.bloodGlucose.high $>, 3$ ),

(bodySen.in.bloodGlucose.low, <end $>, 4$ ),

(end, $<>, 5$ ),

(bodySen.in.bloodGlucose.nor, <end >,4),

(end, $<>, 5$ ),

(bodySen.in.bloodGlucose.threshold, <intravenousNeedle.out.administer.insulin.1, intravenousNeedle.out.administer.insulin.2,

intravenousNeedle.out.administer.insulin.3,intravenousNeedle.out.administer.insulin.4,intravenousNeedle.out.administer.insulin.5>,4),

(intravenousNeedle.out.administer.insulin.1,<phone.out.call.cNeighbor $>, 5$ ),

(phone.out.call.cNeighbor, $<$ phone.out.call.cFamily $>$,6),

(phone.out.call.cFamily, <phone.in.call.ack $>, 7$ ),

(phone.in.call.ack, <end $>, 8$ ),

(end, <>, 9),

(intravenousNeedle.out.administer.insulin.2,<phone.out.call.cNeighbor $>, 5$ ),

(phone.out.call.cNeighbor, $<$ phone.out.call.cFamily $>$,6),

(phone.out.call.cFamily, $<$ phone.in.call.ack $>, 7$ ),

(phone.in.call.ack, <end $>, 8$ ),

(end, $<>, 9$ ) ,

(intravenousNeedle.out.administer.insulin.3,<phone.out.call.cNeighbor $>, 5$ ),

(phone.out.call.cNeighbor, $<$ phone.out.call.cFamily $>$,6),

(phone.out.call.cFamily, <phone.in.call.ack $>, 7$ ),

(phone.in.call.ack, <end $>, 8$ ),

(end, <>, 9) ,

(intravenousNeedle.out.administer.insulin.4,<phone.out.call.cNeighbor $>, 5$ ),

(phone.out.call.cNeighbor, $<$ phone.out.call.cFamily $>$,6),

(phone.out.call.cFamily, <phone.in.call.ack >, 7), 
(phone.in.call.ack, <end $>, 8$ ),

(end, $<>, 9$ ) ,

(intravenousNeedle.out.administer.insulin.5,<phone.out.call.cNeighbor $>, 5$ ),

(phone.out.call.cNeighbor, $<$ phone.out.call.cFamily $>, 6$ ),

(phone.out.call.cFamily, <phone.in.call.ack>, 7),

(phone.in.call.ack, <end $>, 8$ ),

(end, <>, 9),

(bodySen.in.bloodGlucose.high, <intravenousNeedle.out.administer.insulin.1, intravenousNeedle.out.administer.insulin.2,

intravenousNeedle.out.administer.insulin.3,intravenousNeedle.out.administer.insulin.4,intravenousNeedle.out.administer.insulin.5>,4),

(intravenousNeedle.out.administer.insulin.1,<phone.out.call.cNeighbor $>, 5$ ),

(phone.out.call.cNeighbor, <phone.out.call.cFamily $>, 6$ ),

(phone.out.call.cFamily, <phone.in.call.ack $>, 7$ ),

(phone.in.call.ack, <end $>, 8$ ),

(end, $<>, 9)$,

(intravenousNeedle.out.administer.insulin.2,<phone.out.call.cNeighbor $>, 5$ ),

(phone.out.call.cNeighbor, $<$ phone.out.call.cFamily $>, 6$ ),

(phone.out.call.cFamily, <phone.in.call.ack>, 7),

(phone.in.call.ack, <end $>, 8$ ),

(end, $<>, 9$ ),

(intravenousNeedle.out.administer.insulin.3,<phone.out.call.cNeighbor $>, 5$ ),

(phone.out.call.cNeighbor, $<$ phone.out.call.cFamily $>, 6$ ),

(phone.out.call.cFamily, $<$ phone.in.call.ack $>, 7$ ),

(phone.in.call.ack, <end $>, 8$ ),

(end, $<>, 9$ ),

(intravenousNeedle.out.administer.insulin.4,<phone.out.call.cNeighbor $>, 5$ ),

(phone.out.call.cNeighbor, $<$ phone.out.call.cFamily $>, 6$ ),

(phone.out.call.cFamily, <phone.in.call.ack $>, 7$ ),

(phone.in.call.ack, <end $>, 8$ ),

(end, $<>, 9$ ),

(intravenousNeedle.out.administer.insulin.5,<phone.out.call.cNeighbor $>, 5$ ),

(phone.out.call.cNeighbor, $<$ phone.out.call.cFamily $>, 6$ ),

(phone.out.call.cFamily, <phone.in.call.ack $>, 7$ ),

(phone.in.call.ack, <end >,8),

(end, $<>, 9$ ),

(bodySen.in.bodyTemp.39,<bodySen.in.bloodGlucose.low, bodySen.in.bloodGlucose.nor, bodySen.in.bloodGlucose.threshold,

bodySen.in.bloodGlucose.high $>, 3$ ),

(bodySen.in.bloodGlucose.low, <intravenousNeedle.out.administer.antipyretic.1, intravenousNeedle.out.administer.antipyretic.2,

intravenousNeedle.out.administer.antipyretic.3,intravenousNeedle.out.administer.antipyretic.4,intravenousNeedle.out.administer.antipyretic.5>,4),

(intravenousNeedle.out.administer.antipyretic.1,<phone.out.call.cNeighbor $>, 5$ ),

(phone.out.call.cNeighbor, $<$ phone.out.call.cFamily $>, 6$ ),

(phone.out.call.cFamily, <phone.in.call.ack>, 7),

(phone.in.call.ack, <end $>, 8$ ),

(end, $<>, 9)$,

(intravenousNeedle.out.administer.antipyretic.2,<phone.out.call.cNeighbor $>, 5$ ),

(phone.out.call.cNeighbor, $<$ phone.out.call.cFamily $>, 6$ ),

(phone.out.call.cFamily, <phone.in.call.ack>, 7),

(phone.in.call.ack, <end >,8),

(end, $<>, 9)$,

(intravenousNeedle.out.administer.antipyretic.3,<phone.out.call.cNeighbor $>, 5$ ),

(phone.out.call.cNeighbor, $<$ phone.out.call.cFamily $>, 6$ ),

(phone.out.call.cFamily, <phone.in.call.ack>, 7),

(phone.in.call.ack, <end $>, 8$ ),

(end, $<>, 9$ ) ,

(intravenousNeedle.out.administer.antipyretic.4,<phone.out.call.cNeighbor $>, 5$ ),

(phone.out.call.cNeighbor, $<$ phone.out.call.cFamily $>, 6$ ),

(phone.out.call.cFamily, <phone.in.call.ack $>, 7$ ),

(phone.in.call.ack, <end $>, 8$ ),

(end, $<>, 9$ )

(intravenousNeedle.out.administer.antipyretic.5,<phone.out.call.cNeighbor $>, 5$ ), 
(phone.out.call.cNeighbor, $<$ phone.out.call.cFamily $>, 6$ ),

(phone.out.call.cFamily, <phone.in.call.ack>, 7),

(phone.in.call.ack, <end $>, 8)$,

(end, $<>, 9)$,

(bodySen.in.bloodGlucose.nor, <intravenousNeedle.out.administer.antipyretic.1, intravenousNeedle.out.administer.antipyretic.2,

intravenousNeedle.out.administer.antipyretic.3,intravenousNeedle.out.administer.antipyretic.4,intravenousNeedle.out.administer.antipyretic.5>,4), (intravenousNeedle.out.administer.antipyretic.1,<phone.out.call.cNeighbor $>, 5$ ),

(phone.out.call.cNeighbor, $<$ phone.out.call.cFamily $>, 6$ ),

(phone.out.call.cFamily, <phone.in.call.ack $>, 7$ ),

(phone.in.call.ack, <end $>, 8$ ),

(end, $<>, 9$ ),

(intravenousNeedle.out.administer.antipyretic.2,<phone.out.call.cNeighbor $>, 5$ ),

(phone.out.call.cNeighbor, $<$ phone.out.call.cFamily $>, 6$ ),

(phone.out.call.cFamily, <phone.in.call.ack>, 7),

(phone.in.call.ack, <end $>, 8$ ),

(end, $<>, 9$ ) ,

(intravenousNeedle.out.administer.antipyretic.3,<phone.out.call.cNeighbor $>, 5$ ),

(phone.out.call.cNeighbor, $<$ phone.out.call.cFamily $>, 6$ ),

(phone.out.call.cFamily, <phone.in.call.ack>, 7),

(phone.in.call.ack, <end $>, 8$ ),

(end, <>, 9),

(intravenousNeedle.out.administer.antipyretic.4,<phone.out.call.cNeighbor $>, 5$ ),

(phone.out.call.cNeighbor, $<$ phone.out.call.cFamily $>, 6$ ),

(phone.out.call.cFamily, <phone.in.call.ack>, 7),

(phone.in.call.ack, <end $>, 8$ ),

(end, $<>, 9$ ) ,

(intravenousNeedle.out.administer.antipyretic.5,<phone.out.call.cNeighbor $>, 5$ ),

(phone.out.call.cNeighbor, $<$ phone.out.call.cFamily $>, 6$ ),

(phone.out.call.cFamily, <phone.in.call.ack>, 7),

(phone.in.call.ack, <end $>, 8$ ),

(end, $<>, 9$ ),

(bodySen.in.bloodGlucose.threshold, <intravenousNeedle.out.administer.antipyretic.1, intravenousNeedle.out.administer.antipyretic.2,

intravenousNeedle.out.administer.antipyretic.3,intravenousNeedle.out.administer.antipyretic.4,intravenousNeedle.out.administer.antipyretic.5>,4),

(intravenousNeedle.out.administer.antipyretic.1,<phone.out.call.cNeighbor $>, 5$ ),

(phone.out.call.cNeighbor, $<$ phone.out.call.cFamily $>, 6$ ),

(phone.out.call.cFamily, <phone.in.call.ack>, 7),

(phone.in.call.ack, <end $>, 8$ ),

(end, $<>, 9$ ),

(intravenousNeedle.out.administer.antipyretic.2,<phone.out.call.cNeighbor $>, 5$ ),

(phone.out.call.cNeighbor, $<$ phone.out.call.cFamily $>, 6$ ),

(phone.out.call.cFamily, <phone.in.call.ack>, 7),

(phone.in.call.ack, <end $>, 8$ ),

(end, $<>, 9$ ),

(intravenousNeedle.out.administer.antipyretic.3,<phone.out.call.cNeighbor $>, 5$ ),

(phone.out.call.cNeighbor, $<$ phone.out.call.cFamily $>, 6$ ),

(phone.out.call.cFamily, $<$ phone.in.call.ack $>, 7$ ),

(phone.in.call.ack, <end $>, 8$ ),

(end, <>, 9),

(intravenousNeedle.out.administer.antipyretic.4,<phone.out.call.cNeighbor $>, 5$ ),

(phone.out.call.cNeighbor, $<$ phone.out.call.cFamily $>, 6$ ),

(phone.out.call.cFamily, <phone.in.call.ack>, 7),

(phone.in.call.ack, <end $>, 8$ ),

(end, $<>, 9$ )

(intravenousNeedle.out.administer.antipyretic.5,<phone.out.call.cNeighbor $>, 5$ ),

(phone.out.call.cNeighbor, $<$ phone.out.call.cFamily $>, 6$ ),

(phone.out.call.cFamily, <phone.in.call.ack>, 7),

(phone.in.call.ack, <end $>, 8$ ),

(end, $<>, 9$ )

(bodySen.in.bloodGlucose.high, <intravenousNeedle.out.administer.antipyretic.1, intravenousNeedle.out.administer.antipyretic.2, 
intravenousNeedle.out.administer.antipyretic.3,intravenousNeedle.out.administer.antipyretic.4,intravenousNeedle.out.administer.antipyretic.5>,4), (intravenousNeedle.out.administer.antipyretic.1,<phone.out.call.cNeighbor $>, 5$ ), (phone.out.call.cNeighbor, $<$ phone.out.call.cFamily $>$,6),

(phone.out.call.cFamily, <phone.in.call.ack>, 7),

(phone.in.call.ack, <end $>, 8$ ),

(end, <>, 9),

(intravenousNeedle.out.administer.antipyretic.2,<phone.out.call.cNeighbor $>, 5$ ),

(phone.out.call.cNeighbor, $<$ phone.out.call.cFamily $>, 6$ ),

(phone.out.call.cFamily, $<$ phone.in.call.ack $>, 7$ ),

(phone.in.call.ack, <end $>8$ ),

(end, <>, 9),

(intravenousNeedle.out.administer.antipyretic.3,<phone.out.call.cNeighbor $>, 5$ ),

(phone.out.call.cNeighbor, $<$ phone.out.call.cFamily $>$,6),

(phone.out.call.cFamily, $<$ phone.in.call.ack $>, 7$ ),

(phone.in.call.ack, <end $>, 8$ ),

(end, <>, 9) ,

(intravenousNeedle.out.administer.antipyretic.4,<phone.out.call.cNeighbor $>, 5$ ),

(phone.out.call.cNeighbor, $<$ phone.out.call.cFamily $>$,6),

(phone.out.call.cFamily, $<$ phone.in.call.ack $>, 7$ ),

(phone.in.call.ack, $<$ end $>, 8$ ),

(end, <>, 9),

(intravenousNeedle.out.administer.antipyretic.5,<phone.out.call.cNeighbor $>, 5$ ),

(phone.out.call.cNeighbor, $<$ phone.out.call.cFamily $>$,6),

(phone.out.call.cFamily, $<$ phone.in.call.ack $>$, 7),

(phone.in.call.ack, $<$ end $>, 8$ ),

(end, <>, 9),

(voiceRec.in.cough.false, <imageRec.in.numbnessFace.true, imageRec.in.numbnessFace.false>,2),

(imageRec.in.numbnessFace.true, <imageRec.in.fainting.true, imageRec.in.fainting.false>,3),

(imageRec.in.fainting.true, <intravenousNeedle.out.administer.painkiller.1, intravenousNeedle.out.administer.painkiller.2,

intravenousNeedle.out.administer.painkiller.3,intravenousNeedle.out.administer.painkiller.4,intravenousNeedle.out.administer.painkiller.5>,4), (intravenousNeedle.out.administer.painkiller.1, <phone.out.call.cNeighbor>, 5),

(phone.out.call.cNeighbor, $<$ phone.out.call.c911>, 6),

(phone.out.call.c911, <phone.out.call.cFamily $>, 7$ ),

(phone.out.call.cFamily, <phone.in.call.ack $>, 8$ ),

(phone.in.call.ack, $<$ phone.in.call.ack $>, 9$ ),

(phone.in.call.ack, <end $>, 10$ ),

(end, <>, 11),

(intravenousNeedle.out.administer.painkiller.2, <phone.out.call.cNeighbor $>, 5$ ),

(phone.out.call.cNeighbor, <phone.out.call.c911>, 6),

(phone.out.call.c911, <phone.out.call.cFamily >, 7),

(phone.out.call.cFamily, $<$ phone.in.call.ack $>, 8$ ),

(phone.in.call.ack, $<$ phone.in.call.ack $>$,9),

(phone.in.call.ack, <end $>, 10$ ),

(end, $<>, 11$ ),

(intravenousNeedle.out.administer.painkiller.3, <phone.out.call.cNeighbor >, 5),

(phone.out.call.cNeighbor, <phone.out.call.c911>, 6),

(phone.out.call.c911, <phone.out.call.cFamily $>, 7$ ),

(phone.out.call.cFamily, <phone.in.call.ack $>, 8$ ),

(phone.in.call.ack, $<$ phone.in.call.ack $>, 9$ ),

(phone.in.call.ack, $<$ end $>, 10$ ),

(end, <>, 11),

(intravenousNeedle.out.administer.painkiller.4, $<$ phone.out.call.cNeighbor $>, 5$ ),

(phone.out.call.cNeighbor, <phone.out.call.c911>, 6),

(phone.out.call.c911, <phone.out.call.cFamily $>$, 7),

(phone.out.call.cFamily, <phone.in.call.ack $>, 8$ ),

(phone.in.call.ack, <phone.in.call.ack >,9),

(phone.in.call.ack, <end $>, 10$ ),

(end, <>, 11),

(intravenousNeedle.out.administer.painkiller.5, <phone.out.call.cNeighbor>, 5), 
(phone.out.call.cNeighbor, <phone.out.call.c911>, 6),

(phone.out.call.c911, <phone.out.call.cFamily $>$, 7),

(phone.out.call.cFamily, $<$ phone.in.call.ack $>, 8$ ),

(phone.in.call.ack, <phone.in.call.ack $>$,9),

(phone.in.call.ack, <end $>, 10$ ),

(end, $<>, 11$ ),

(imageRec.in.fainting.false,<intravenousNeedle.out.administer.painkiller.1, intravenousNeedle.out.administer.painkiller.2,

intravenousNeedle.out.administer.painkiller.3,intravenousNeedle.out.administer.painkiller.4,intravenousNeedle.out.administer.painkiller.5>,4),

(intravenousNeedle.out.administer.painkiller.1, <phone.out.call.cNeighbor $>, 5$ ),

(phone.out.call.cNeighbor, <phone.out.call.c911>, 6),

(phone.out.call.c911, < phone.out.call.cFamily $>, 7$ ),

(phone.out.call.cFamily, <phone.in.call.ack $>, 8$ ),

(phone.in.call.ack, $<$ phone.in.call.ack $>, 9$ ),

(phone.in.call.ack, <end $>, 10$ ),

(end, $<>, 11$ ),

(intravenousNeedle.out.administer.painkiller.2, $<$ phone.out.call.cNeighbor $>, 5$ ),

(phone.out.call.cNeighbor, <phone.out.call.c911>, 6),

(phone.out.call.c911, <phone.out.call.cFamily >, 7),

(phone.out.call.cFamily, $<$ phone.in.call.ack $>, 8$ ),

(phone.in.call.ack, $<$ phone.in.call.ack $>, 9$ ),

(phone.in.call.ack, $<$ end $>, 10$ ),

(end, $<>, 11$ ),

(intravenousNeedle.out.administer.painkiller.3, <phone.out.call.cNeighbor >, 5),

(phone.out.call.cNeighbor, <phone.out.call.c911>, 6),

(phone.out.call.c911, $<$ phone.out.call.cFamily $>, 7$ ),

(phone.out.call.cFamily, $<$ phone.in.call.ack $>, 8$ ),

(phone.in.call.ack, $<$ phone.in.call.ack $>, 9$ ),

(phone.in.call.ack, <end $>, 10$ ),

(end, $<>, 11$ ),

(intravenousNeedle.out.administer.painkiller.4, $<$ phone.out.call.cNeighbor $>, 5$ ),

(phone.out.call.cNeighbor, <phone.out.call.c911>, 6),

(phone.out.call.c911, <phone.out.call.cFamily $>, 7$ ),

(phone.out.call.cFamily, $<$ phone.in.call.ack $>, 8$ ),

(phone.in.call.ack, $<$ phone.in.call.ack $>, 9$ ),

(phone.in.call.ack, $<$ end $>, 10$ ),

(end, <>, 11),

(intravenousNeedle.out.administer.painkiller.5, <phone.out.call.cNeighbor $>, 5$ ),

(phone.out.call.cNeighbor, <phone.out.call.c911>, 6),

(phone.out.call.c911, $<$ phone.out.call.cFamily $>, 7$ ),

(phone.out.call.cFamily, <phone.in.call.ack $>, 8$ ),

(phone.in.call.ack, $<$ phone.in.call.ack $>, 9$ ),

(phone.in.call.ack, <end $>, 10$ ),

(end, $<>, 11$ ),

(imageRec.in.numbnessFace.false,<imageRec.in.fainting.true, imageRec.in.fainting.false>,3),

(imageRec.in.fainting.true, <intravenousNeedle.out.administer.painkiller.1, intravenousNeedle.out.administer.painkiller.2,

intravenousNeedle.out.administer.painkiller.3, intravenousNeedle.out.administer.painkiller.4,intravenousNeedle.out.administer.painkiller.5>,4),

(intravenousNeedle.out.administer.painkiller.1, <phone.out.call.cNeighbor >, 5),

(phone.out.call.cNeighbor, <phone.out.call.c911>, 6),

(phone.out.call.c911, <phone.out.call.cFamily>, 7),

(phone.out.call.cFamily, <phone.in.call.ack $>, 8$ ),

(phone.in.call.ack, <phone.in.call.ack $>$,9),

(phone.in.call.ack, $<$ end $>, 10$ ),

(end, $<>, 11$ ),

(intravenousNeedle.out.administer.painkiller.2, <phone.out.call.cNeighbor>, 5),

(phone.out.call.cNeighbor, <phone.out.call.c911>, 6),

(phone.out.call.c911, <phone.out.call.cFamily>, 7),

(phone.out.call.cFamily, <phone.in.call.ack $>, 8$ ),

(phone.in.call.ack, $<$ phone.in.call.ack $>, 9$ ),

(phone.in.call.ack,<end $>, 10$ ), 
(end, $<>, 11$ ),

(intravenousNeedle.out.administer.painkiller.3, <phone.out.call.cNeighbor $>, 5$ ), (phone.out.call.cNeighbor, <phone.out.call.c911>, 6),

(phone.out.call.c911, <phone.out.call.cFamily >, 7),

(phone.out.call.cFamily, $<$ phone.in.call.ack $>, 8$ ),

(phone.in.call.ack, $<$ phone.in.call.ack $>9$ ),

(phone.in.call.ack, <end $>, 10$ ),

(end, $<>, 11$ ),

(intravenousNeedle.out.administer.painkiller.4, $<$ phone.out.call.cNeighbor $>, 5$ ), (phone.out.call.cNeighbor, <phone.out.call.c911>, 6),

(phone.out.call.c911, < phone.out.call.cFamily >, 7),

(phone.out.call.cFamily, $<$ phone.in.call.ack $>, 8$ ),

(phone.in.call.ack, $<$ phone.in.call.ack $>, 9$ ),

(phone.in.call.ack,<end $>, 10$ ),

(end, $<>, 11$ )

(intravenousNeedle.out.administer.painkiller.5, <phone.out.call.cNeighbor >, 5), (phone.out.call.cNeighbor, <phone.out.call.c911>, 6),

(phone.out.call.c911, <phone.out.call.cFamily >, 7),

(phone.out.call.cFamily, $<$ phone.in.call.ack $>, 8$ ),

(phone.in.call.ack, <phone.in.call.ack $>9$ ),

(phone.in.call.ack,<end $>, 10$ ),

(end, $<>, 11$ ),

(imageRec.in.fainting.false, $<$ end $>, 4$ ),

(end, $<>, 5$ ) 


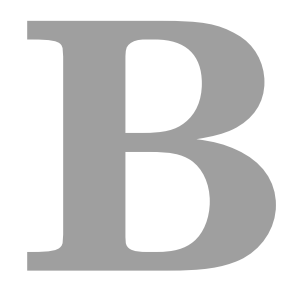

\section{Gnutella helper functions}

This appendix shows the functions used in the I/O process Servent (Section 5.3).

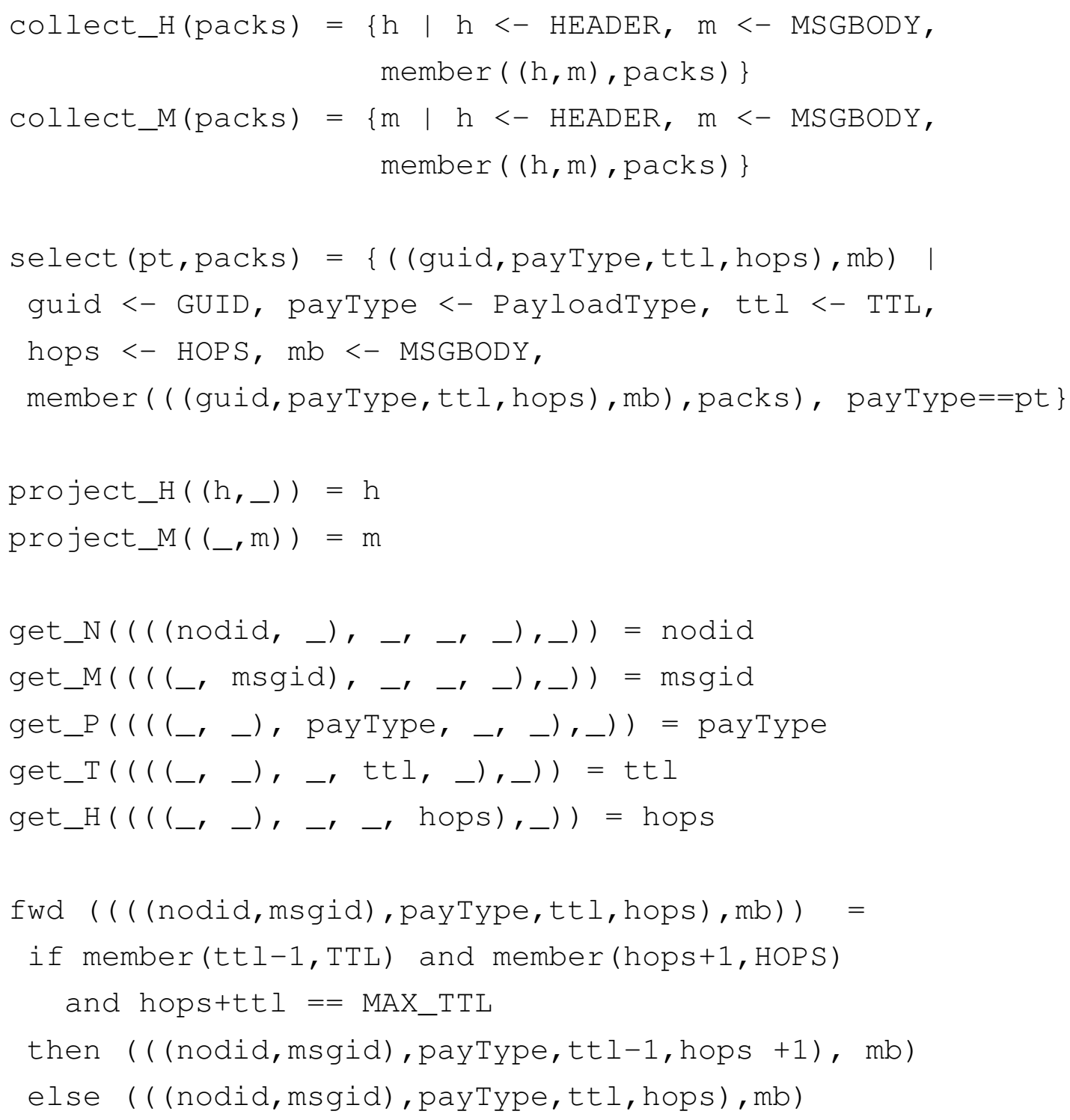




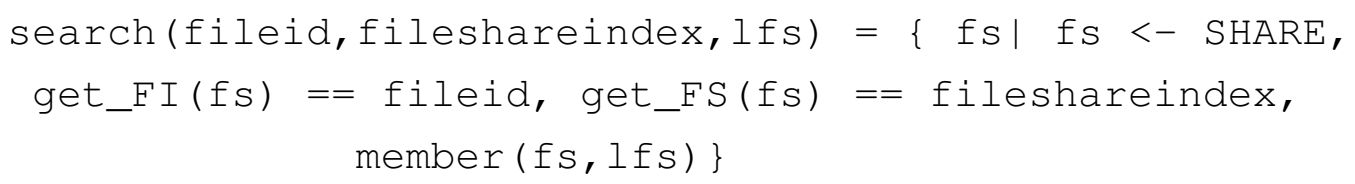




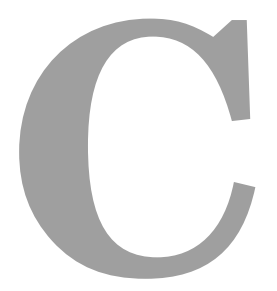

\section{CSP syntax and notation}

This appendix presents the relevant syntax and notation of CSP (ROSCOE, 1998) used in this work.

Table C.1: CSP processes

\begin{tabular}{|c|c|c|}
\hline \multicolumn{3}{|c|}{ Processes } \\
\hline CSP & $C S P_{M}$ & description \\
\hline STOP & STOP & termination \\
\hline SKIP & SKIP & successful termination \\
\hline$c \rightarrow P$ & $C->P$ & prefix \\
\hline$P ; Q$ & $\mathrm{P} ; \mathrm{Q}$ & sequential composition \\
\hline$P \backslash X$ & $\mathrm{P} \backslash \mathrm{X}$ & hiding \\
\hline$P \square Q$ & $P[] Q$ & external choice \\
\hline$P \sqcap Q$ & $P|\sim| Q$ & internal choice \\
\hline$b \& P$ & $b \& P$ & boolean guard \\
\hline if $b$ then $P$ else $Q$ & if $\mathrm{b}$ then $\mathrm{P}$ else $\mathrm{Q}$ & if-then-else \\
\hline$P[[a / b]]$ & $P[[a<-b]]$ & renaming \\
\hline$P \| Q$ & $P|| \mid Q$ & interleaving \\
\hline$P \|_{a} Q$ & $P \quad[|a|] Q$ & alphabetized parallel \\
\hline$\square e: X @ P$ & {[]$\quad e \quad: X @ P$} & replicated external choice $(X \neq \emptyset)$ \\
\hline$\sqcap e: X @ P$ & $|\sim|$ e $: X$ \& $P$ & replicated internal choice $(X \neq \emptyset)$ \\
\hline$\| e: X @ P$ & || $\mid e: X \& P$ & replicated interleave \\
\hline$\| e: X @\left[X^{\prime}\right] P$ & ||$e: X \&\left[X^{\prime}\right] P$ & replicated alphabetized parallel \\
\hline
\end{tabular}


Table C.2: Traces

\begin{tabular}{|c|c|c|}
\hline \multicolumn{2}{|c|}{ Trace notation } \\
\hline CSP & $C S P_{M}$ & description \\
\hline \hline$\Sigma^{*}$ & & set of all finite traces over $\Sigma$ \\
\hline\langle\rangle & $<>$ & the empty trace \\
\hline$t^{\wedge} s$ & $t^{\wedge} \mathrm{s}$ & concatenation of traces \\
\hline$s \leq t$ & $\mathrm{~s}<=\mathrm{t}$ & $\equiv u \cdot s^{\wedge} u=t$ (prefix order) \\
\hline$\# s$ & \# & length of $s$ \\
\hline & & $t-\langle\rangle=t$ \\
$t-s$ & $t-\mathrm{s}$ & \langle\rangle$^{\wedge} s=\langle\rangle$ \\
& & $\left.\left(\left\langle e_{1}\right\rangle^{\wedge} t\right)-\left(\left\langle e_{2}\right\rangle^{\wedge} s\right)=\langle\rangle_{1}\right\rangle^{\wedge} s=t-\left(t-\left(\left\langle e_{2}\right\rangle^{\wedge} s\right)\right) \mid e_{1} \neq e_{2}$ \\
\hline
\end{tabular}

Table C.3: Events

\begin{tabular}{|c|c|c|}
\hline \multicolumn{2}{|c|}{ Communication/Sets } \\
\hline CSP & $\operatorname{CSP}_{M}$ & description \\
\hline \hline$\Sigma$ & all & alphabet of all communications \\
\hline$\{|c|\}$ & $\{|\mathrm{C}|\}$ & the events communicated through channel $c$ \\
\hline$X \backslash Y$ & diff $(\mathrm{X}, \mathrm{Y})$ & $\{e \mid e \in X \wedge e \notin Y\}$ \\
\hline$\checkmark$ & & (tick) termination event \\
\hline$\tau$ & & (tau) invisible event \\
\hline$\Sigma^{\checkmark}$ & & $\Sigma \cup\{\checkmark\}$ \\
\hline$\Sigma^{\checkmark, \tau}$ & & $\Sigma \cup\{\checkmark, \tau\}$ \\
\hline
\end{tabular}

